\title{
Graphical Assessment of the Prediction Capability \\ of Response Surface Designs \\ by
}

Ann Giovannitti-Jensen

Dissertation submitted to the Faculty of the

Virginia Polytechnic Institute and State University

in partial fulfillment of the requirements for the degree of

Doctor of Philosophy

in

Statistics

APPROVED:

/ Raymond H. Myers, 'Chairman

Klaus H. Hinkelmann

Marvin Lentner

Jeffrey B. Birch

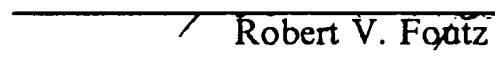

September 23, 1987

Blacksburg, Virginia 


\section{Graphical Assessment of the Prediction Capability \\ of Response Surface Designs}

by

Ann Giovannitti-Jensen

Raymond H. Myers, Chairman

Statistics

(ABSTRACT)

A response surface analysis is concerned with the exploration of a system in order to determine the behavior of the response of the system as levels of certain factors which influence the response are changed. It is often of particular interest to predict the response in some region of the allowable factor values and to find the optimal operating conditions of the system.

In an experiment to search for the optimum response of a surface it is advantageous to predict the response with equal, or nearly equal, precision at all combinations of the levels of the variables which represent locations which are the same distance from the center of the experimental region. Measures of the quality of prediction at locations on the surface of a hypersphere are presented in this thesis. These measures are used to form a graphical method of assessing the overall prediction capability of an experimental design throughout the region of interest.

Rotatable designs give equal variances of predicted values corresponding to locations on the same sphere. In this case, the center of the sphere coincides with the center of the rotatable design. However, there is a need for a method to quantify the prediction capability on spheres for nonrotatable designs. The spherical variance is a measure of the average prediction variance at locations on the surface of a sphere. The spherical variance obtained with a design provides an assessment of how well the response is being estimated on the average at locations which are the same distance from the region center. This thesis introduces two measures which describe the dispersion in the variances of the predicted responses at all locations on the surface of a sphere. These prediction 
variance dispersion (PVD) measures are used to evaluate the ability of a design to estimate the response with consistent precision at locations which are the same distance from the region center. The PVD measures are used in conjunction with the spherical variance to assess the prediction capability of a design.

A plot of the spherical variance and the maximum and minimum prediction variances for locations on a sphere against the radius of the sphere gives a comprehensive picture of the behavior of the prediction variances throughout a region, and, hence, of the quality of the predicted responses, obtained with a particular design. Such plots are used to investigate and compare the prediction capabilities of certain response surface designs currently available to the researcher. The plots are also used to investigate the robustness of a design under adverse experimental conditions and to determine the effects of taking an additional experimental run on the quality of the predicted responses. 


\section{Acknowledgements}

I would like to express my sincere gratitude and appreciation to Dr. Raymond Myers for all his help and sharing of ideas and especially for his understanding, patience and faith.

The generous time, comments and suggestions given by Drs. Klaus Hinkelmann, Marvin Lentner, Jeffrey Birch and Robert Foutz are also appreciated. I also thank Dr. Bradley Skarpness for his interest in the problem and his efforts in finding an appropriate computer algorithm. To I extend many thanks for many hours kindly spent. I am very grateful as well to for her skillful typing of part of this manuscript and for her immeasurable help with script. To ; thanks for the lettering and for being there.

I am thankful for the many fine people I have met and worked with here, and especially for and for their friendship and comradeship through the years. To my parents and family for their faith and support and for teaching me the most important lessons in life - no amount of thanks is great enough. 


\section{Table of Contents}

I. Introduction $\ldots \ldots \ldots \ldots \ldots \ldots \ldots \ldots \ldots \ldots \ldots \ldots \ldots \ldots \ldots \ldots \ldots$ I

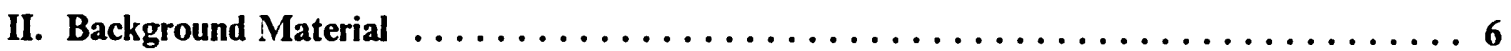

2.1 RSM - An Experimental Strategy $\ldots \ldots \ldots \ldots \ldots \ldots \ldots \ldots \ldots \ldots \ldots \ldots \ldots \ldots \ldots \ldots$

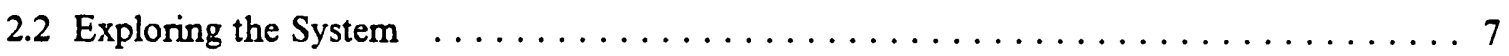

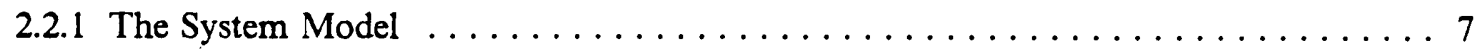

2.2.2 The Experimental Design $\ldots \ldots \ldots \ldots \ldots \ldots \ldots \ldots \ldots \ldots \ldots$

2.2.3 A Convenient Form of the Model $\ldots \ldots \ldots \ldots \ldots \ldots \ldots \ldots \ldots \ldots$

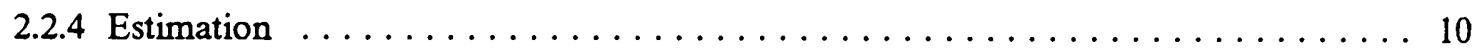

2.3 Some Design Properties and Criteria $\ldots \ldots \ldots \ldots \ldots \ldots \ldots \ldots \ldots \ldots \ldots \ldots \ldots \ldots \ldots \ldots$

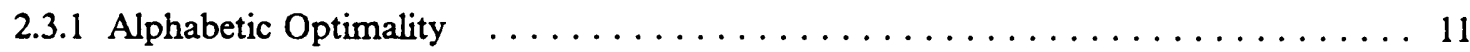

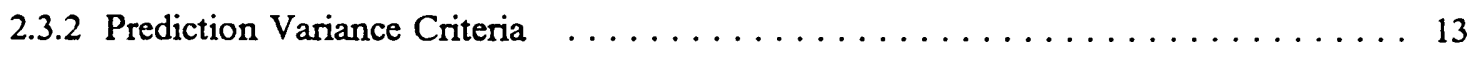

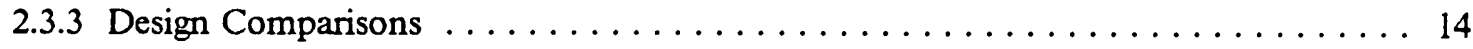

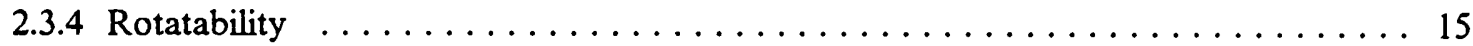

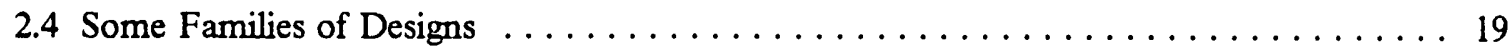

2.4.1 Two Level Factorial Designs $\ldots \ldots \ldots \ldots \ldots \ldots \ldots \ldots \ldots \ldots \ldots \ldots$

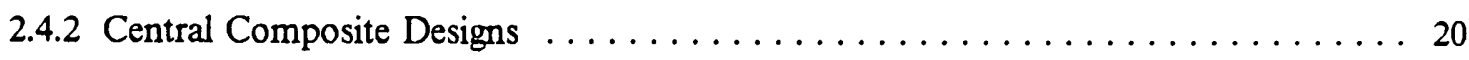




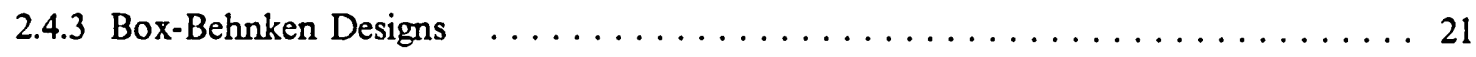

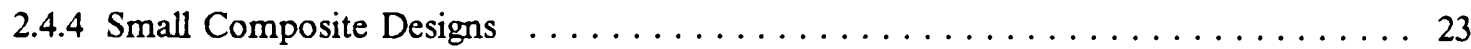

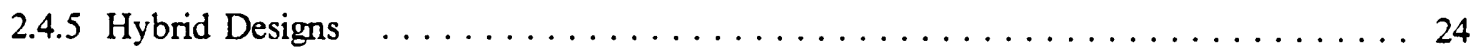

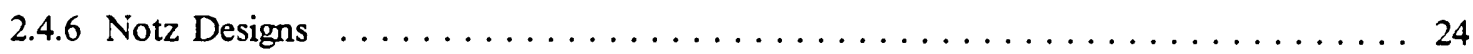

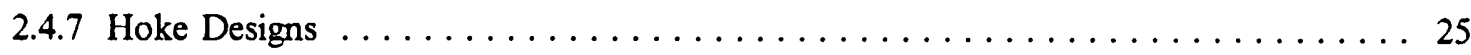

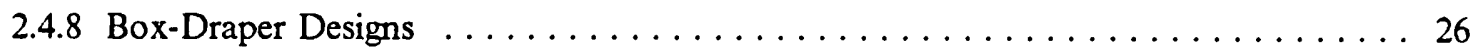

2.4.9 Computer Generated Minimum Point D-Optimal Designs $\ldots \ldots \ldots \ldots \ldots 27$

III. Assessment of Prediction Capability on Spheres $\ldots \ldots \ldots \ldots \ldots \ldots \ldots \ldots \ldots$

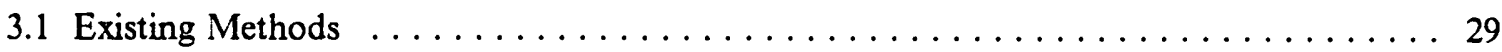

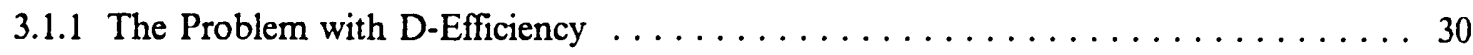

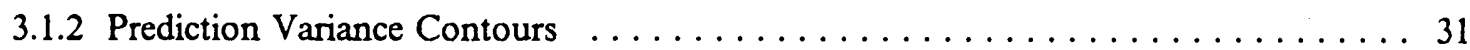

3.1.3 Prediction Variance Pictures for Rotatable Designs $\ldots \ldots \ldots \ldots \ldots \ldots \ldots . \ldots 32$

3.1.4 Prediction Variance Pictures for Non-Rotatable Designs $\ldots \ldots \ldots \ldots \ldots \ldots 35$

3.2 Prediction Variance Dispersion Measures $\ldots \ldots \ldots \ldots \ldots \ldots \ldots \ldots \ldots \ldots \ldots \ldots \ldots$

3.2.1 The Need for Prediction Variance Dispersion Measures $\ldots \ldots \ldots \ldots \ldots \ldots .41$

3.2.2 Measuring the Dispersion in Prediction Variances $\ldots \ldots \ldots \ldots \ldots \ldots \ldots 44$

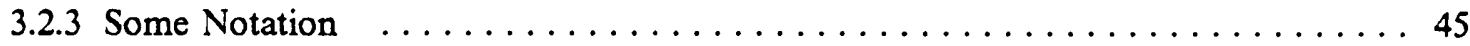

3.2.4 The Range of Variances on the Surface of a Hypersphere $\ldots \ldots \ldots \ldots \ldots 51$

3.2.5 The VofV on the Surface of a Hypersphere $\ldots \ldots \ldots \ldots \ldots \ldots \ldots \ldots \ldots$

3.3 Illustrations of the Use of the Spherical Variance and PVD Measures $\ldots \ldots \ldots \ldots 63$

3.3.1 A Plot Depicting Prediction Capability on Spheres $\ldots \ldots \ldots \ldots \ldots \ldots \ldots$

3.3.2 Illustration of the Effects of Loss of Design Points .................64 64

3.3.3 Illustration of the Effects of Improper Design Settings $\ldots \ldots \ldots \ldots \ldots \ldots 66$

3.3.4 Illustration of the Effects of Augmenting a Point to the Design ........... 76

IV. Properties of the Spherical Variance and PVD Measures $\ldots \ldots \ldots \ldots \ldots \ldots \ldots$

4.1 Trends in the Prediction Variance Functions $\ldots \ldots \ldots \ldots \ldots \ldots \ldots \ldots \ldots \ldots . \ldots \ldots$ 
4.1.1 Trends in the Spherical Variance as $\mathrm{r}$ Increases $\ldots \ldots \ldots \ldots \ldots$

4.1.2 Trends in the RofV as $\mathrm{r}$ Increases $\ldots \ldots \ldots \ldots \ldots \ldots \ldots \ldots \ldots$

4.1.3 Trends in the VofV as $\mathrm{r}$ Increases $\ldots \ldots \ldots \ldots \ldots \ldots \ldots$

4.2 Invariance Properties of the Spherical Variance and PVD Measures $\ldots \ldots \ldots \ldots$

4.2.1 Invariance to the Scale of the Design Variables $\ldots \ldots \ldots \ldots \ldots \ldots$

4.2.2 Invariance to the Center of the Design Variables $\ldots \ldots \ldots \ldots \ldots \ldots$

4.2.3 Invariance to Orthogonal Transformation of the Independent Variables . . . . . . 98

4.2.4 Invariance of RofV and VofV to the Number of Center Points of the Design $\ldots 101$

4.3 A New Criterion for Design Augmentation $\ldots \ldots \ldots \ldots \ldots \ldots \ldots \ldots \ldots \ldots \ldots \ldots \ldots$

4.4 The Dilemma over the Choice of Design Center $\ldots \ldots \ldots \ldots \ldots \ldots$

V. Graphical Assessment of the Prediction Capability of Certain Second Order Designs . . 110

5.1 The Family of Central Composite Designs $\ldots \ldots \ldots \ldots \ldots \ldots \ldots \ldots \ldots \ldots$

5.1.1 Comparisons of Prediction Capability in Three Variable CCDs $\ldots \ldots \ldots \ldots 11$

5.1.2 Comparison of a Box-Behnken Design with Two CCDs $\ldots \ldots \ldots \ldots \ldots$

5.1.3 Robustness of a Rotatable CCD to Loss of Design Points $\ldots \ldots \ldots \ldots \ldots$

5.2 Minimum and Nearly Minimum Point Designs $\ldots \ldots \ldots \ldots \ldots \ldots$

5.2.1 A Comparison of Small Composite and Hybrid Designs $\ldots \ldots \ldots \ldots \ldots$

5.2.2 A Comparison of Some Small D-Efficient Designs $\ldots \ldots \ldots \ldots \ldots \ldots$

5.2.3 Comparisons of Computer Generated D-Optimal Designs $\ldots \ldots \ldots \ldots \ldots$

5.3 Measuring Deviation from Rotatability $\ldots \ldots \ldots \ldots \ldots \ldots \ldots \ldots \ldots \ldots \ldots \ldots \ldots \ldots$

5.3.1 The Deviation from Rotatability of a $3^{2}$ Factorial Design $\ldots \ldots \ldots \ldots \ldots$

5.3.2 The Deviation from Rotatability of Hybrid Designs $\ldots \ldots \ldots \ldots \ldots \ldots$

VI. Concluding Remarks and Further Research $\ldots \ldots \ldots \ldots \ldots \ldots \ldots \ldots \ldots$

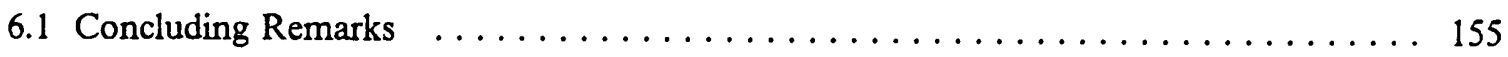

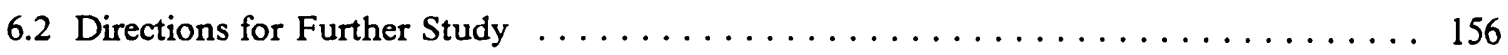




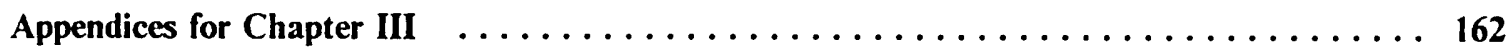

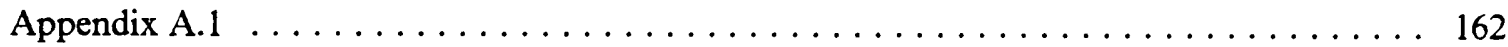

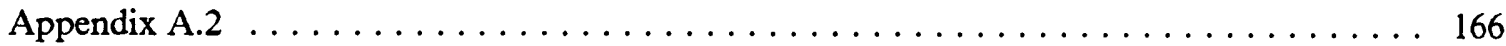

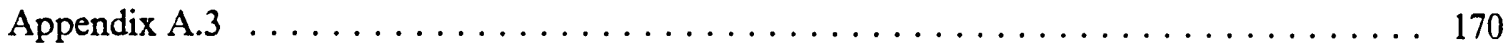

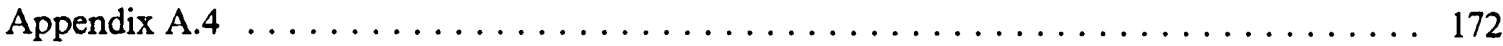

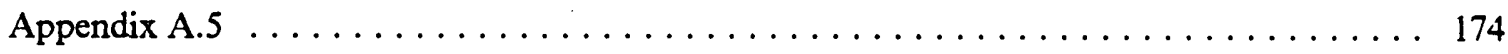

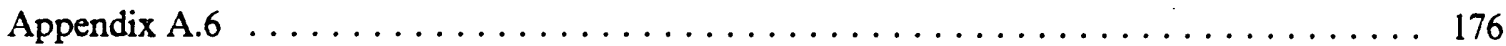

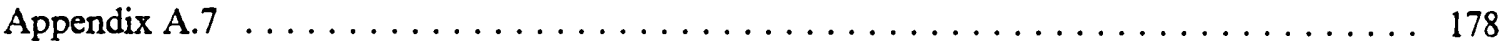

Appendices for Chapter IV $\ldots \ldots \ldots \ldots \ldots \ldots \ldots \ldots \ldots \ldots \ldots \ldots \ldots \ldots \ldots \ldots$

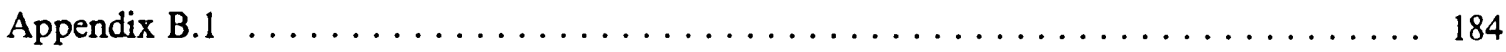

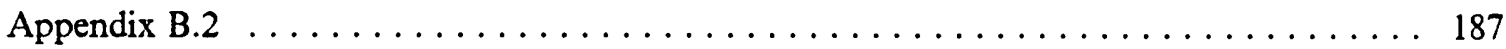

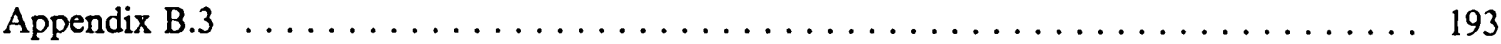

Vita $\ldots \ldots \ldots \ldots \ldots \ldots \ldots \ldots \ldots \ldots \ldots \ldots \ldots \ldots \ldots \ldots \ldots \ldots \ldots \ldots \ldots \ldots$

Table of Contents 


\section{List of Tables}

Table 1. Design Points for Computer Generated Second Order

Designs with Three Factors at Three Levels . . . . . . . . . 28

Table 2. Spherical Variances for Example Designs of

Section 3.1.5

Table 3. Locations for Calculation of Prediction Variance for

Example Designs of Section 3.1.5 . . . . . . . . . . . . 43

Table 4. Prediction Variances for the Locations

Given in Table 3. . . . . . . . . . . . . . . . 43

Table 5. Roquemore's Hybrid Designs in Three Variables . . . . . . . . . 130 


\section{List of Figures}

Figure 1. Prediction Variance Contours for a $2^{2}$ Factorial Design . . . . . . . 33

Figure 2. Prediction Variance Contours for a $2^{2}$ Factorial Design

with Improper Settings . . . . . . . . . . . . . . . . 34

Figure 3. Comparison of Prediction Variances for Two Rotatable Designs . . . . . 36

Figure 4. Comparison of Spherical Variances for Three First Order Designs - . . . 42

Figure 5. Spherical Variance, Maximum and Minimum Prediction Variances for a $2^{3}$ Factorial with 2 Missing and No Center Points . . . . . . 67

Figure 6. Spherical Variance, Maximum and Minimum Prediction Variances for a $2^{3}$ Factorial with 2 Missing and 2 Center Points . . . . . . . 68

Figure 7. Locations of Maximum Prediction Variance on Spheres for a $2^{3}$ with 2 Missing and No Center Points . . . . . . . . . . 69

Figure 8. Comparison of Spherical Variances for $2^{4}$ Factorial Designs . . . . . . 73

Figure 9. Spherical Variance, Maximum and Minimum Prediction Variances for a $2^{4}$ Design with Improper Settings in 4 Variables, 1-10 Percent Off . . . . . . 74

Figure 10. Spherical Variance, Maximum and Minimum Prediction Variances for a $2^{4}$ Design with Improper Settings in 2 Variables, 25-50 Percent Off . • . . 75

Figure 11. Spherical Variance, Maximum and Minimum Prediction Variances for the 2-Level First Order Design of Section 3.3.4

Figure 12. Locations of Maximum Prediction Variances on Spheres for the 2-Level First Order Design of Section 3.3.4 . . . . . . . . 79

Figure 13. Spherical Variance, Maximum and Minimum Prediction Variances for the 2-Level First Order Design of Section 3.3.4 A Design Point Augmented at the Location of Maximum Variance . . . . 80

Figure 14. Plot of $r$ Against $\mu$. . . . . . . . . . . . . . 87 
Figure 15. Comparison of Spherical Variances for CCDs with One Center Point . . . 113

Figure 16. Spherical Variance, Maximum and Minimum Prediction Variances for a CCD with $\alpha=1.35$ and One Center Point . . . . . . . . 114

Figure 17. Spherical Variance, Maximum and Minimum Prediction Variances for a CCD with $\alpha=1.00$ and One Center Point . . . . . . . . 115

Figure 18. Comparison of Spherical Variances for CCDs with $\alpha=1.682$ and a Varying Number of Center Points . . . . . . . . . 117

Figure 19. Comparison of Spherical Variances for CCDs with Three Center Points . . 119

Figure 20. Spherical Variance, Maximum and Minimum Prediction Variances for a Box-Behnken Design with One Center Point . . . . . . . . 120

Figure 21. Comparison of Spherical Variances for Two CCDs and a Box-Behnken Design -. One Center Point . . . . . . . . . . . . . . .

Figure 2la. Comparison of Spherical Variances for Two CCDs and a Box-Behnken Design -- Four Center Points . . . . . . . . . . . . 123

Figure 22. Spherical Variance, Maximum and Minimum Prediction Variances for a CCD with One Design Point Lost . . . . . . . . . . 125

Figure 23. Spherical Variance, Maximum and Minimum Prediction Variances for a CCD with Two Design Points Lost . . . . . . . . . . 126

Figure 24. Locations of Maximum Prediction Variance on Spheres for a CCD with Two Design Points Lost . . . . . . . . . . 127

Figure 25. Comparison of Spherical Variances for Two Hybrid Designs and an SCD $\quad$ - 131

Figure 26. Spherical Variance, Maximum and Minimum Prediction Variances for an SCD with $\alpha=1.732$ and 1 Center Point . . . . . . . . 132

Figure 27. Spherical Variance, Maximum and Minimum Prediction Variances for a Hybrid 310 Design with 1 Center Point . . . . . . . . . 133

Figure 28. Spherical Variance, Maximum and Minimum Prediction Variances for a Hybrid 311A Design with 1 Center Point . . . . . . . . . 134

Figure 29. Comparison of Spherical Variances for Some Small D-Efficient Designs . • 137

Figure 30. Spherical Variance, Maximum and Minimum Prediction Variances for the Box-Draper Design . . . . . . . . . . . . 138

Figure 31. Spherical Variance, Maximum and Minimum Prediction Variances for the Hoke D2 Design . . . . . . . . . . . . . 139

Figure 32. Spherical Variance, Maximum and Minimum Prediction Variances for the Notz Design . . . . . . . . . . . . . . 140

Figure 33. Comparison of Spherical Variances for Computer Generated Designs . . - 142 
Figure 34. Spherical Variance, Maximum and Minimum Prediction Variances for the Mitchell-Bayne MB10 Design . . . . . . . . . . 143

Figure 35. Spherical Variance, Maximum and Minimum Prediction Variances for the Welch WDI0 Design . . . . . . . . . . . . 144

Figure 36. Spherical Variance, Maximum and Minimum Prediction Variances for the Welch WC11 Design ............... . 145

Figure 37. Spherical Variance, Maximum and Minimum Prediction Variances for the $3^{2}$ Factorial Design . . . . . . . . . . . . 147

Figure 38. Spherical Variance, Maximum and Minimum Prediction Variances for the Hybrid 310 Design . . . . . . . . . . . 150

Figure 39. Spherical Variance, Maximum and Minimum Prediction Variances for the Hybrid 311A Design 151

Figure 40. Spherical Variance, Maximum and Minimum Prediction Variances for the Hybrid 311B Design 


\section{Chapter I}

\section{Introduction}

Researchers in many areas such as engineering, chemistry, medicine and agronomy often explore a system to determine the behavior of the response of the system as levels of certain factors which influence the response are changed. The system under investigation may be, for example, a manufacturing process or a biological system. In many such situations the researcher also wishes to find levels of the influential variables which produce optimal behavior in the response. The collection of procedures used to explore the behavior of the response and to define the optimal operating conditions of the system in which the response is a random variable is called Response Surface Methodology (RSM).

As a function of the influential, or independent, variables present in the system, the response is represented as a surface in the space of the independent variables. The true functional relationship between the response and the independent variables is usually unknown or complicated. Thus, the response surface is often modelled as a simple function of the independent variables. The model serves to describe the general behavior of the responses and to predict individual values of the response at specified combinations of the levels of the independent variables. 
The determination of the nature of the response surface is accomplished through experimentation and observation of the response. The choice of the experimental design has a major impact on the quality of the inferences drawn from the experiment. A well designed experiment is a valuable tool in the investigation of the response of a system.

There are many characteristics of a design which may be considered when selecting a response surface design. Some design criteria are discussed in Chapter II. All the available criteria by which a design may be judged are single-valued measures of some design characteristic, for example, the generalized variance of the coefficients of the response surface model. Since the estimation of the response is of primary importance in a response surface analysis, the focus of this research is on the assessment of the prediction capability of an experimental design in some region of the allowable factor values.

As an example of how the quality of the predicted values may be assessed, consider a system in which there is one independent variable, say $x$, which is thought to influence the behavior of the response, $y$. Suppose the response function is approximated by a simple linear regression model,

$$
y=\beta_{0}+\beta_{1} x+\varepsilon
$$

for values of $\mathrm{x}$ in the interval $[+1,-1]$. Further, suppose that only three experimental runs are available for experimentation.

One possible design plan the researcher may adopt is to take two of the observations at $x=1$ and the remaining observation at $\mathrm{x}=-1$. This is the D-optimal strategy in this situation. That is, the generalized variance of the coefficients $\beta_{0}$ and $\beta_{1}$ is as small as possible with this design for $\mathrm{x} \in[+1,-1]$. The average variance of the predicted values integrated over locations $\mathrm{x}$ in the interval $[+1,-1]$ obtained with this design is 0.5 . Note that all variances reported here are in the units of the error variance associated with the model. The actual prediction variances range from 1 at $x=-1$, to $1 / 3$ when estimating the response at $x=1 / 3$, the average of the settings of $x$ 
used in the experiment. In fact, the response which occurs when $\mathrm{x}$ has a value of +1 is estimated with a variance which is half of the variance of the estimated response at $x=-1$.

Alternatively, the researcher may choose to take an experimental run at at each of the levels $+1,0$ and -1 of the independent variable $x$. Although the average prediction variance for valucs of $\mathrm{x}$ in the interval $[+1,-1]$ is the same as that obtained with the D-optimal design, the accuracy in the estimated responses at most levels of $\mathrm{x}$ is quite different from that obtained with the previous design. The second design strategy yields prediction variances ranging from $5 / 6$, the same when the level of $x$ is at +1 or -1 , to $1 / 3$ at $x=0$. The responses at locations which are the same distance from $x=0$, the center of the set of design points, are estimated with equal precision with this design.

The nature of the prediction variances is easily graphed in the single variable case, as in the sketch below. The graph provides a ready comparison of the prediction capabilities for the two design plans.

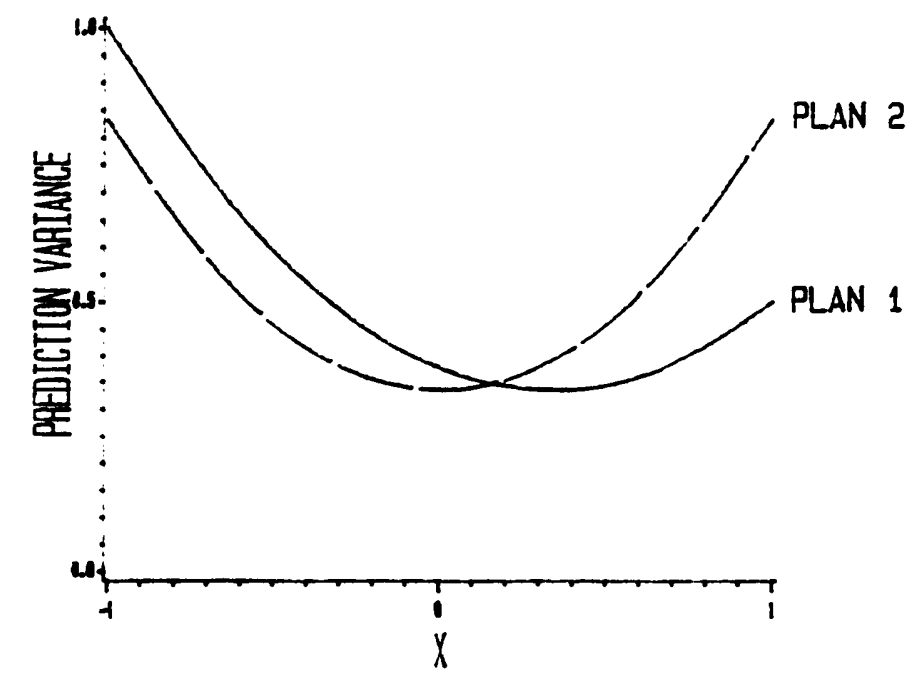


In this simple example one might easily have deduced the different behaviors of the prediction variances in the interval for each design. However, the nature of the prediction variances is much more complicated and less intuitive when two or more variables are known to influence the response. There is a need in experimental situations involving any number of variables to look at the prediction variances throughout the region of interest in order to learn where the responses are estimated well and poorly, and to judge the accuracy of the estimated responses obtained with the design. Clearly, no single-valued measure constructed to describe the prediction capability of a design can give a comprehensive description of the prediction variances throughout a region.

The impetus behind this research is the need in a response surface analysis to have a general understanding of the nature of the prediction variances in the region of the independent variables for the experimental design. To aid the researcher in achieving an overall view of the prediction capability of a design, criteria will be presented which, when viewed as a unit, depict the behavior of the prediction variances throughout some region for a specific design and model. It will be possible to display the variance criteria in a graph, thus facilitating the assessment of the quality of the predicted responses attained with the design.

The general exploration of the response surface and search for the optimum response usually proceeds in all directions from the center of the region of interest. Thus, it is natural to attempt to describe the composition of the set of prediction variances at all locations which are the same distance from the region center. These locations are points on the surface of a hypersphere about the region center. The prediction variance criteria developed through this research characterize the behavior of the variances of the estimated responses on spheres in the region. The quality of prediction in some region may be surveyed through the graphical representation of the prediction variance criteria as functions of the radius of the sphere.

The prediction variance criteria are introduced in Chapter III. The criteria measure the average prediction variance on a sphere and the dispersion in the prediction variances on a sphere for a specific experimental design and response surface model. The criteria will be implemented so that 
one may be able to illustrate the effects of a change in the experimental conditions on the prediction variances obtained with a design.

The behavior of the measures as the radius of the sphere increases, that is, as the response is estimated further from the region center, is considered in Chapter IV. Other properties of the measures are also established in this chapter. In particular, the invariance of these measures to the units of the design variables used in the analysis is investigated.

Lastly, the measures are applied to some response surface designs currently available to the researcher. In Chapter $\mathrm{V}$ plots of the criteria against the radius of the sphere are used to investigate and compare the prediction capabilities of these designs. It will be seen that the graphical method of assessing the prediction capability of an experimental design provides a comprehensive picture of the quality of information about the response obtainable from that design. 


\section{Chapter II}

\section{Background Material}

\subsection{RSM - An Experimental Strategy}

Response surface methodology has its roots in an industrial laboratory. G. E. P. Box (1954) and others, (see also, Box and Wilson (1951) and Box and Youle (1955)), originated the development of what is now called response surface methodology (RSM) as a consequence of exploring the behavior of the yield of a chemical process and trying to improve the yield by control of pertinent variables in the process. They recognized that experimentation is a sequential process in this situation and adopted an iterative plan which consisted of (1) postulating a model for the response, (2) selecting an experimental design, and (3) analyzing the resulting data. This cycle was continued until satisfactory results were obtained. Since that time, RSM has evolved through efforts by researchers to explore and improve systems such as chemical processes.

$\mathrm{RSM}$ is a unified collection of theories and procedures which provide an experimental strategy for analyzing evolving problems in a laboratory setting. Included in the collection are the principles 
and theories of experimental design, optimization techniques, estimation methods and statistical inference.

The experimental design, which is the focus of this thesis, is perhaps the most important aspect of a response surface analysis. A good experimental design along with the proper analysis of the data will provide the researcher with reliable information about the system. Without a good design, however, even the most ingenious analysis will leave many of the questions posed by the researcher unanswered.

\subsection{Exploring the System}

\subsubsection{The System Model}

Let $y$ denote the response of the system which depends on the influential factors $\xi_{1}, \xi_{2}, \ldots, \xi_{k}$ present in the system. It is assumed that the experimenter has control over the values of $\xi_{1}, \xi_{2}, \ldots, \xi_{k}$ in the experiment and that these variables are continuous. We can define the system mathematically as

$$
y=f\left(\xi_{1}, \xi_{2}, \ldots, \xi_{k}\right)+\varepsilon
$$

where $\varepsilon$ is the usual random error term. The functional form of $f$ is unknown. However, it can be approximated by a low order polynomial in some region of the allowable values of the independent variables $\xi_{1}, \xi_{2}, \ldots, \xi_{k}$. It is convenient to express the polynomial model in terms of the design variables, $x_{1}, x_{2}, \ldots, x_{k}$, of the system, rather than the variables $\xi_{1}, \xi_{2}, \ldots, \xi_{k}$, which are expressed in the natural units of the system. The design variables are simply centered and scaled versions (simple linear transformations) of the natural variables. In an experiment where $N$ meas- 
urements of the response are taken at the levels $\xi_{i 1}, \xi_{i 2}, \ldots, \xi_{i N}$ say of the independent variable $\xi_{i}$, the corresponding design variable $x_{i}$ is defined by

$$
x_{i u}=\frac{\xi_{i u}-\bar{\xi}_{i}}{s_{l}}, \quad u=1,2, \ldots, N
$$

where $\bar{\xi}_{i} \quad=\sum_{u=1}^{N} \xi_{i u} / N$ and $s_{i}$ is an appropriate scale term.

A first order model for the observed response in terms of the design variables is

$$
y=\beta_{0}+\sum_{i=1}^{k} \beta_{i} x_{i}+\varepsilon
$$

and a second order polynomial model is of the form

$$
y=\beta_{0}+\sum_{i=1}^{k} \beta_{i} x_{i}+\sum_{i=1}^{k} \beta_{i i} x_{i}^{2}+\sum_{\substack{i=1 \\ i<j}}^{k} \sum_{j=1}^{k} \beta_{i j} x_{i} x_{j}+\varepsilon
$$

Often a first order model is employed in a small region of the design variables where there is thought to be little curvature in the response function. Such a model might be used to locally approximate the response function in the initial stages of the experiment to find a new region in the space of the independent variables where the response is higher (or lower). In larger regions or as the search draws nearer to the optimum response a second order model is used to approximate the response function in that region.

First and second order models are simple in form and often provide adequate approximations to the true response function in some region. Although there are many other models which could be adopted, these are the most frequently chosen empirical models used to investigate a system. Thus, only first and second order models will be considered in this research. However, much of what is accomplished here can be extended to other empirical or theoretical models. 


\subsubsection{The Experimental Design}

In the experiment $N$ observations of the response are made at $N$ combinations, not all necessarily distinct, of the levels of the $\mathrm{k}$ independent variables $x_{1}, x_{2}, \ldots, x_{k}$. The set of combinations of levels, or settings, chosen to be analyzed is the experimental design. The individual settings are design points. In our discussion there are $N$ design points. The matrix consisting of the $N$ design points is called the design matrix.

The experimental design is a valuable tool in the investigation of a response surface. The careful choice of a design is crucial to the quality and reliability of the information obtained from the experiment. Several criteria an experimenter might use in choosing an experimental design are described in the next section.

\subsubsection{A Convenient Form of the Model}

The functional relationship between the observed responses and the input variables in the experiment can be described in matrix form by

$$
y=X \underline{B}+\underline{\varepsilon}
$$

where $y=\left(\begin{array}{lllll}y_{1} & y_{2} & \ldots & y_{N}\end{array}\right)^{\prime}$ is the vector of observed responses,

$B$ is the $p \times 1$ vector of parameters which appear in the chosen model,

$\underline{\varepsilon}=\left(\begin{array}{lllll}\varepsilon_{1} & \varepsilon_{2} & \ldots & \varepsilon_{N}\end{array}\right)^{\prime}$ is the vector of random errors associated with $y$. For our purposes the errors are assumed to be independent, identically distributed random variables with mean 0 and variance $\sigma^{2}$.

$X$ is an $N \times$ p matrix which reflects the experimental design. $X$ will be referred to as the model-matrix to distinguish it from the design matrix. 
The nature of $X$ depends on the choice of model. For example, the parameters of a first order model are $\beta_{0}$ and $\beta_{1}, \ldots, \beta_{k}$. The vector of parameters is then, $\beta=\left(\beta_{0} \beta_{1} \ldots \beta_{k}\right)^{\prime}$. The matrix $X$ corresponding to a first order model is

$$
X=\left[\begin{array}{ccccc}
1 & x_{11} & x_{12} & \ldots & x_{1 k} \\
1 & x_{21} & x_{22} & \ldots & x_{2 k} \\
\cdot & \cdot & \cdot & \cdot & \cdot \\
\cdot & \cdot & \cdot & \cdot & \cdot \\
\cdot & \cdot & . & . & \cdot \\
1 & x_{N 1} & x_{N 2} & \ldots & x_{N k}
\end{array}\right]
$$

where $x_{i j}$ represents the level of variable $x_{j}$ for the $\mathrm{i}$-th setting of the factors used in the experiment, $\mathrm{i}=1,2, \ldots, N$ and $\mathrm{j}=1,2, \ldots, \mathrm{k}$. There is a natural extension to higher order models.

\subsubsection{Estimation}

The vector of unknown parameters is estimated using ordinary least squares methods by

$$
\hat{\beta}=\left(X^{\prime} X\right)^{-1} X^{\prime} y
$$

The variance/covariance matrix of the estimated coefficients under the assumption that $\underline{\varepsilon} \sim\left(\underline{0}, \sigma^{2} I\right)$ is

$$
\operatorname{Var}(\hat{\beta})=\sigma^{2}\left(X^{\prime} X\right)^{-1}
$$

The estimated values of the response at the design points are

$$
\hat{y}=\left(\begin{array}{llll}
\hat{y}_{1} & \hat{y}_{2} & \ldots & \hat{y}_{N}
\end{array}\right)^{\prime}=X \hat{\underline{\beta}}
$$

and the estimated value of the response at any location $x$ in the region of interest is 


$$
\hat{y}(\underline{x})=\underline{x^{\prime}} \hat{\underline{B}}
$$

Thus, the variance of a predicted value of the response at a point $\underline{x}$ is given by

$$
\operatorname{Var} \hat{y}(\underline{x}))=\sigma^{2} \underline{x}^{\prime}\left(X^{\prime} X\right)^{-1} \underline{x}
$$

Note that for a first order model $x$ is of the form $\left(\begin{array}{lllll}1 & x_{1} & x_{2} & \ldots & x_{k}\end{array}\right)^{\prime}$ and for a second order model $\underline{x}=\left(\begin{array}{llllllllllll}1 & x_{1} & \ldots & x_{k} & x_{1}^{2} & \ldots & x_{k}^{2} & x_{1} x_{2} & \ldots & x_{k-1} x_{k}\end{array}\right)^{\prime}$.

\subsection{Some Design Properties and Criteria}

An experimenter may judge the quality of an experimental design against many criteria. Some design properties and criteria which are often considered when choosing a design are presented in this section. Naturally, different criteria may lead to the selection of different designs. The criterion and, therefore, the design used should be consistent with the focus of the experiment. Of course, there are times when compromises among several criteria have to be made.

\subsubsection{Alphabetic Optimality}

Often the researcher is interested in providing a workable model for the response of the system. Usually, the response is modelled as a linear or a quadratic function of the independent variables or factors present in the system. In any case, as the goal is to come up with the best model for the response, a design is chosen which will yield the best estimates of the true parameters in the selected model. Thus, a design which provides a good fit to the model will minimize, in some sense, the variances of the estimated coefficients. 
One variance criterion which has been considered in the literature is the generalized variance of the coefficients. The generalized variance is defined to be the determinant of the variance /covariance matrix of the vector of estimated coefficients, $\hat{\beta}$.

$$
D=|\operatorname{Var}(\hat{\underline{B}})|=\left|\sigma^{2}\left(X^{\prime} X\right)^{-1}\right|
$$

where $|\cdot|$ denotes the determinant. A design which is the best with respect to the generalized variance is one which minimizes $\left|\left(X^{\prime} X\right)^{-1}\right|$, or, equivalently, maximizes $\left|\left(X^{\prime} X\right)\right|$. Such a design is said to be D-optimal.

Kiefer and Wolfowitz $(1959,1960)$ define a D-optimal design as a continuous probability measure which determines the proportion of experimental runs which should be taken at each of the settings in the space of all permissable design settings to minimize D. As a consequence, a D-optimal design cannot always be achieved in practice. However, realistic designs which are approximations to such D-optimal designs, and which are frequently nearly D-optimal are used in practice, (see, e.g., Nalimov, et. al. (1970)). For a more detailed overview of D-optimality and D-optimal designs the interested reader is referred to St. John and Draper (1975).

Many other optimality criteria have been studied. All focus on making the variance/covariance matrix of $\hat{\mathfrak{B}}$, or, more precisely, some real-valued functional form of it, small. Some of these are listed below.

1. An A-optimal design is one which minimizes the trace of $\left(X^{\prime} X\right)^{-1}$.

This is equivalent to minimizing the sum of the variances of the estimated coefficients.

2. An E-optimal design is one which minimizes the maximum eigenvalue of $\left(X^{\prime} X\right)^{-1}$.

3. A G-optimal design is one which minimizes the maximum 
value of $\sigma^{2} \underline{x}^{\prime}\left(X^{\prime} X\right)^{-1} \underline{x}$

the variance of the predicted response, over all

locations in some region of the allowable factor values.

\subsubsection{Prediction Variance Criteria}

The G-optimality criterion pertains specifically to the quality of the predicted values of the response in a certain region of the factor values. The prediction capability of a design is of particular importance in response surface problems. Recall that RSM is primarily concerned with the determination of the behavior of the response. Hence, in RSM at least, useful experimental designs are ones which predict well in a specified region of the allowable factor values. It is reasonable to expect that a design chosen to best fit the data to the selected model will also predict the response well at the design points. However, it is also desirable that the response be well estimated throughout the region of interest. The integrated prediction variance, IV, first described by Box and Draper $(1959,1963)$, is a measure of the prediction capability of a design in a specified region. Essentially, IV is the average prediction variance in a region $\mathrm{R}$. For a design $D$ and region $\mathrm{R}$, the IV is defined as

$$
\begin{aligned}
I V(D) & =\frac{N K}{\sigma^{2}} \int_{R} \operatorname{Var}(\hat{y}(\underline{x})) d \underline{x} \\
& =N K \int_{R} x^{\prime}\left(X^{\prime} X\right)^{-1} \underline{x} d \underline{x}
\end{aligned}
$$

where $N=$ the total number of design points and $K^{-1}=\int_{R} d \underline{x}$ is the volume of the region $\mathrm{R}$. In practice, the region $R$ is taken to be a sphere or cube in the space of the independent variables. The design which minimizes IV is considered to be best with respect to this criterion. The factor of $N$ takes into account the efficiency of the design. Thus, a design with a smaller number of points is preferred over a more costly design with similar prediction capability. By integrating the mean 
square error of a predicted response over a region, Box and Draper also investigated the effects of bias due to inadequate model specification on the prediction capability of a design.

\subsubsection{Design Comparisons}

Designs defined in the same region of the design variables can be compared to the D-optimal and G-optimal designs by considering their D- and G-efficiencies respectively, (Atwood (1969)).

D-efficiency measures the efficiency of a given design for a model containing $p$ parameters relative to the D-optimal designs for the same model. Thus, if $X$ is the model-matrix for a design, say $D^{\bullet}$, with $N$ design points and $X_{D}$ is the model-matrix for the D-optimal design of size $N_{D}$, the D-efficiency for design $D^{\bullet}$ is defined to be

$$
\operatorname{D-efficiency}\left(D^{*}\right)=\left[\frac{\left|\left(X^{\prime} X\right) / N\right|}{\left|\left(X_{D^{\prime}} X_{D}\right) / N_{D}\right|}\right]^{\frac{1}{p}}
$$

Note that the D-efficiency is the ratio of the generalized variances for the two designs scaled by their respective sizes. A design which has a D-efficiency of 1.0 is a D-optimal design.

The G-efficiency of a design $D^{\bullet}$ with $N$ design points and model-matrix $X$ relative to the Goptimal design with $N_{G}$ design points and model-matrix $X_{G}$ is

$$
\mathrm{G}-\operatorname{efficiency}\left(D^{*}\right)=\frac{d_{G}}{d_{\max }}=\frac{p}{d_{\max }}
$$

where $d_{G}=\max _{\underline{x} \in \mathbb{R}} N_{G} \underline{x}^{\prime}\left(X_{G} X_{G}\right)^{-1} \underline{x}=p$ for a G-optimal design, (Kiefer and Wolfowitz (1960)) and $d_{\max }=\max _{\underline{x} \in \mathrm{R}} N \underline{x}^{\prime}\left(X^{\prime} X\right)^{-1} \underline{x}$. Here $\mathrm{R}$ denotes the region of the design variables under consideration. 
G-efficiency then is the ratio of the maximum prediction variances in some region for the two designs. A G-optimal design has a G-efficiency of 1.0 since, in that case, $d_{\max }=d_{G}$, the smallest maximum prediction variance among the designs considered.

Many studies have been done to compare the D- and G-efficiencies within certain classes of designs. See, for example, Nalimov, et. al. (1970), Box and Draper (1971) and Lucas (1976). However, few studies have appeared in the literature which consider the integrated variance criterion as a means of comparing competing designs, (Draper (1982) and also Box (1982)).

\subsubsection{Rotatability}

In response surface problems, accurate prediction of the response is very important. Estimation of the optimal response is of special interest in such problems. Since the optimal operating conditions of the system are unknown, one generally begins the search for the optimum in all directions from the center of the design. Consequently, it is advantageous to predict equally well or, nearly equally well, at all locations that are the same distance from the center of the design. That is, the variance of the estimated response at a location should not depend on the direction of the location but only on the distance from the center of the design. So, for all points on a kdimensional hypersphere of radius $r$, i.e., points $\underline{x}$ such that $\sum_{i=1}^{k} x_{i}^{2}=r^{2}$, require $\operatorname{Var}(\hat{y}(\underline{x}))=g\left(r^{2}\right) \sigma^{2}$. This design property is termed rotatability, (see Box and Hunter (1957)). This property is particularly suited to designs used in the exploration of a response surface where the behavior of the response on spheres is of particular interest and the independent variables are continuous.

Rotatability is a property of the experimental design and can be characterized in terms of the elements of the moment matrix $N^{-1} X^{\prime} X$ corresponding to the design. Formally, the moment matrix contains the design moments given by 


$$
\begin{aligned}
& {[\mathrm{i}]=\frac{1}{N} \sum_{u=1}^{N} x_{u i} \quad[\mathrm{ij}]=\frac{1}{N} \sum_{u=1}^{N} x_{u i} x_{u j},} \\
& {[\mathrm{iij}]=\frac{1}{N} \sum_{u=1}^{N} x_{u i}^{2} x_{u j}, \quad[\mathrm{iijj}]=\frac{1}{N} \sum_{u=1}^{N} x_{u i}^{2} x_{u j}^{2}, \quad \text { etc. for } \mathrm{i}, \mathrm{j}=1,2, \ldots, \mathrm{k} .}
\end{aligned}
$$

Moments of the form [i] are called first order moments; [ij] are second order moments, and so on. The moment matrix for a first order model contains design moments of the first and second order only. The design moments through order four are included in the moment matrix when a second order model is fit to the observed response.

Necessary and sufficient conditions for a design used to fit a first order model to be rotatable are that the design moments [i] and [ij] with $\mathrm{i} \neq \mathrm{j}$ are zero, and the pure second moments [ii] have the same value for all $\mathrm{i}=1,2, \ldots, \mathrm{k}$. A first order design which satisfies these conditions is an orthogonal design. In the case of a second order model, a design is rotatable if and only if the odd moments involved, [i], [ii], [iij], [iii] and [iiij] with $\mathrm{i} \neq \mathrm{j}$ are all zero, the pure second moments [ii] are the same for all $\mathrm{i}=1,2, \ldots, \mathrm{k}$, and the pure fourth moments [iii] are three times the mixed fourth moments [iijj] (see Box and Hunter (1957) or Myers (1976) for details). Note that a design which is rotatable in the first order case may not necessarily be rotatable when a second order model is used.

In practice, rotatable designs are often used in response surface problems. However, rotatability can be lost if, for example, one or more of the design runs is lost during the experiment . Also, many design points may be required to achieve rotatability in a design. This is frequently the case when there are a large number of variables describing the response. Thus, the researcher may choose a non-rotatable design to save time and money, particularly at the beginning stages of the experiment.

The use of a rotatable design does not guarantee good estimation of the response of the system. Non-rotatable designs often have better prediction properties than rotatable designs which could be used in the same situation. However, it is desirable to have consistent prediction at lo- 
cations which are the same distance from the center of the design. In recent papers, Khuri (1988) and Draper and Guttman (1988) have set forth measures to determine how near a non-rotatable design is to possessing the rotatability property of equal prediction variances on a sphere.

The measure presented by Khuri (1988) provides a comparison of design moments of a non-rotatable design and a rotatable design of the same size. Both designs are restricted to $[\mathrm{i}]=0$ and $[\mathrm{ii}]=\mathrm{a}$ constant for all $\mathrm{i}=1,2, \ldots, \mathrm{k}$. The comparison of design moments is achieved by calculating the Euclidean distance of a vector containing the moments of the design, call this vector $\underline{u}$, to the corresponding vector for an appropriate rotatable design, say $\underline{v}$. The vector $\underline{v}$ is chosen from the set of all vectors containing the design moments corresponding to rotatable designs of the appropriate size. It is closest to the vector $\underline{u}$ in the sense that the Euclidean distance between the two vectors is smaller than the distance for any other vector belonging to the set. The distance between the two vectors is subtracted from the Euclidean norm of the vector of design moments for the non-rotatable design. The actual measure of rotatability, $\Phi$, is formed as a percentage and is shown below.

$$
\Phi=100\left\{\|\underline{u}\|^{2}-\|\underline{u}-\underline{v}\|^{2}\right\} /\|\underline{u}\|^{2}
$$

Note that if the design under consideration is rotatable, the distance between the design moment vectors $\underline{u}$ and $\underline{v}$ is 0 and $\Phi=100$. A large value of this measure indicates that the moments of the design closely resemble the moments of a rotatable design. In this case, the design is said to be 'near rotatable'.

Draper and Guttman (1988) investigate the nearness to rotatability of a design by considering the shape of a specified prediction variance contour of the design. The contour chosen for examination is an outer contour which passes through at least one point on the edge of the design region. The shape of the outer contour for a design is approximated by a curve of the form

$$
\sum_{i=1}^{k}\left|x_{i}\right|^{\mathrm{m}}=1
$$


The exponent $\mathrm{m}$ is gotten by forcing the curve (2.3.2) through the true variance contour at two points. To simplify the calculation of the prediction variance only second order designs for which the following moment restrictions hold were considered in the development : $[\mathrm{i}]=0,[\mathrm{ii}]=\mathrm{B}$, $[i i i]=C,[i i j j]=D$ for all $\mathrm{i}=1,2, \ldots, k, i \neq j$, and all odd moments through order four are equal to zero. The evaluation of $\mathrm{m}$ would be much more difficult without these moment restrictions.

The value of $\mathrm{m}$ thus found provides an approximate picture of the outer variance contour of the design. This picture is compared to the spherical shape of the prediction variance contours of a rotatable design. By this comparison a judgement can be made regarding how near a nonrotatable design is to possessing the rotatability property of equal prediction variances on spheres. For this reason, $\mathrm{m}$ is termed the index of rotatability.

The property of rotatability pertains to the predicted responses on spheres about the center of the design. The condition that the first moment, [i], be equal to zero for a rotatable design signifies that the design center coincides with the center of the region of the design variables. For this reason, the measures of deviation from rotatability proposed by Khuri and by Draper and Guttman require that the first moment of the design be equal to zero. However, it is usually of interest to investigate the prediction capability of a design on spheres about the center of the region of the design variables. If the experimental design is not centered at the center of the region of interest these indices can not provide an indication of the similarity of the prediction variances on spheres about the region center. The problem of assessing the consistency on spheres as well as the quality of the prediction variances on spheres about the center of the region of interest will be considered in Chapter III. 


\subsection{Some Families of Designs}

This section catalogs some types of designs currently available to the researcher involved in a response surface analysis. The prediction capability of these designs will be investigated as a part of this research. Only $2^{k}$ factorial and fractional factorial designs will be considered when the response surface is approximated by a first order model. All other designs described here are second order designs used for fitting a second order model to the response of the system.

\subsubsection{Two Level Factorial Designs}

Each of the design variables appears at two levels (usually at +1 and -1 ) in a $2^{k}$ factorial design. A full factorial plan consists of all possible combinations of the design variables at these levels. The design matrix for a $2^{2}$ factorial design is presented below.

$$
\left[\begin{array}{rr}
1 & 1 \\
1 & -1 \\
-1 & 1 \\
-1 & -1
\end{array}\right]
$$

These designs and certain fractions of the $2^{k}$ factorials are first order orthogonal and, therefore, rotatable designs. Designs in this class possess many desirable properties; for example, they provide minimum integrated variance of predicted values and minimum generalized variance of the estimated coefficients among first order designs. Certain of them provide minimum mean square error of prediction as well. 


\subsubsection{Central Composite Designs}

The $2^{k}$ factorial designs do not allow for the estimation of the coefficients of a second order model. Central composite designs (ccd) extend the first order factorial designs to be appropriate in the second order model case. A ccd in $\mathrm{k}$ variables is formed by augmenting a $2^{k}$ factorial or fractional factorial design with a set of axial points as follows :

$$
\left[\begin{array}{rrrr} 
\pm 1 & \pm 1 & \ldots & \pm 1 \\
\alpha & 0 & \ldots & 0 \\
-\alpha & 0 & \ldots & 0 \\
0 & \alpha & \ldots & 0 \\
0 & -\alpha & \ldots & 0 \\
. & . & \ldots & \cdot \\
. & \cdot & \ldots & \cdot \\
0 & 0 & \ldots & \alpha \\
0 & 0 & \ldots & -\alpha
\end{array}\right]
$$

Experimental runs taken at the center of the design may also be added. The experimenter chooses the axial value, $\alpha$, and the number of center points, $n_{0}$, to achieve certain design properties. For instance, rotatability can be achieved with the appropriate choice of $\alpha$. As an example, the design matrix for a rotatable ccd in three variables with one center point is 


$$
\left[\begin{array}{rrr}
1 & 1 & 1 \\
1 & 1 & -1 \\
1 & -1 & 1 \\
-1 & 1 & 1 \\
1 & -1 & -1 \\
-1 & 1 & -1 \\
-1 & -1 & 1 \\
-1 & -1 & -1 \\
1.682 & 0 & 0 \\
-1.682 & 0 & 0 \\
0 & 1.682 & 0 \\
0 & -1.682 & 0 \\
0 & 0 & 1.682 \\
0 & 0 & -1.682 \\
0 & 0 & 0
\end{array}\right]
$$

Although ccds in more than two variables require fewer design points than the $3^{k}$ factorial designs, they often require considerably more trials than the minimum necessary for fitting a second order model. The five levels used in the design (unless $\alpha=1.0$ ) may also be probibitive in some experimental situations.

\subsubsection{Box-Behnken Designs}

Box-Behnken designs were introduced as an economical alternative to ccds, (Box and Behnken (1960)). Box-Behnken designs generally require fewer design points and only use three 
levels of the design variables. Like the ccds, however, Box-Behnken designs require many more design points than is needed for estimating the parameters of a second order model.

The designs are constructed from balanced incomplete block structures. For designs in three, four or five variables, portions of the Box-Behnken designs are $2^{2}$ factorial designs in two of the variables and the level of the remaining variables is zero. There are $\left({ }_{2}^{k}\right)$ such portions, one for each pair of variables. For six or more variables the blocks of the Box-Behnken designs are formed from $2^{3}$ factorials in three of the variables with the remaining variables at a level of zero. Not all such blocks are used in the design however. The Box-Behnken design for an experiment in three variables is

$$
\left[\begin{array}{rrr}
-1 & -1 & 0 \\
-1 & 1 & 0 \\
1 & -1 & 0 \\
1 & 1 & 0 \\
-1 & 0 & -1 \\
-1 & 0 & 1 \\
1 & 0 & -1 \\
1 & 0 & 1 \\
0 & -1 & -1 \\
0 & -1 & 1 \\
0 & 1 & -1 \\
0 & 1 & 1 \\
0 & 0 & 0 \\
& &
\end{array}\right]
$$

The remaining designs in this section are all saturated or nearly saturated experimental designs. A saturated or minimum point design is one for which the number of design points required is equal to the minimum number of observations required to estimate the parameters of the model. 
Nearly saturated designs require only a few more points than the minimum number necessary to fit the model. Both saturated and nearly saturated designs are extremely economical. Consequently, they are of interest, especially when observations are expensive or time is short.

\subsubsection{Small Composite Designs}

Small composite designs have the same structure as central composite designs. The factorial portion of a small composite design consists of a fraction of a $2^{k}$ factorial design. It is not a requirement, as it is for a ccd, that the design have all odd design moments equal to zero.

$$
\left[\begin{array}{rrr}
1 & 1 & 1 \\
1 & -1 & -1 \\
-1 & 1 & -1 \\
-1 & -1 & 1 \\
\alpha & 0 & 0 \\
-\alpha & 0 & 0 \\
0 & \alpha & 0 \\
0 & -\alpha & 0 \\
0 & 0 & \alpha \\
0 & 0 & -\alpha \\
0 & 0 & 0 \\
0 & &
\end{array}\right]
$$

This is a small composite design in three variables. The factorial portion is a one-half fraction of a $2^{3}$ factorial array constructed by taking the defining contrast to be $\mathrm{I}=\mathrm{ABC}$. The choice of the axial value, $\alpha$, and the number of center points is left to the discretion of the experimenter. 


\subsubsection{Hybrid Designs}

Roquemore (1976) devised hybrid designs to be near-minimum point designs which are near rotatable and which possess properties similar to ccds. The basic structure of a hybrid design is a ccd in $(k-1)$ variables with an additional column for the $k$-th variable. The three variable hybrid design is of the form

$$
\left[\begin{array}{rrr}
0 & 0 & \alpha_{1} \\
0 & 0 & \alpha_{2} \\
-1 & -1 & \alpha_{3} \\
1 & -1 & \alpha_{3} \\
-1 & 1 & \alpha_{3} \\
1 & 1 & \alpha_{3} \\
\alpha & 0 & \alpha_{4} \\
-\alpha & 0 & \alpha_{4} \\
0 & \alpha & \alpha_{4} \\
0 & -\alpha & \alpha_{4}
\end{array}\right]
$$

In his paper, Roquemore gives several choices for the design parameters $\alpha, \alpha_{1}, \alpha_{2}, a_{3}, \alpha_{4}$ for hybrid designs in three and four variables. In some cases, the factorial or axial portion, or both, of the $(k-1)$ variable ccd may be rotated or scaled.

\subsubsection{Notz Designs}

Notz designs are minimum or nearly minimum point second order designs using three levels of the design variables. They have the form of a $2^{k}$ factorial design, or a specified fraction of it, augmented with a kxk identity matrix. Thus, in three variables a Notz design is 


$$
\left[\begin{array}{rrr}
1 & 1 & 1 \\
1 & 1 & -1 \\
1 & -1 & 1 \\
-1 & 1 & 1 \\
1 & -1 & -1 \\
-1 & 1 & -1 \\
-1 & -1 & 1 \\
-1 & -1 & -1 \\
1 & 0 & 0 \\
0 & 1 & 0 \\
0 & 0 & 1
\end{array}\right]
$$

Notz designs are not rotatable. Their D-efficiencies compare favorably with other minimum point designs when the number of factors is small. The reader is referred to Notz (1982) for a more detailed description of Notz designs.

\subsubsection{Hoke Designs}

Hoke designs are also minimum or nearly minimum point three level designs. Their construction is based on irregular fractions of a $3^{k}$ factorial design generated from sets of partially balanced arrays, (see Hoke (1974) for details). An example of a Hoke design in three variables is 


$$
\left[\begin{array}{rrr}
-1 & -1 & -1 \\
1 & 1 & -1 \\
1 & -1 & 1 \\
-1 & 1 & 1 \\
0 & 0 & -1 \\
0 & -1 & 0 \\
-1 & 0 & 0 \\
1 & -1 & -1 \\
-1 & 1 & -1 \\
-1 & -1 & 1 \\
& &
\end{array}\right]
$$

\subsubsection{Box-Draper Designs}

Box and Draper $(1971,1974)$ implemented a restricted D-optimality criterion to generate these designs. The designs were restricted to be minimum point designs with design points located on or within the unit cube. Subject to these conditions, the design was found which yielded the largest determinant of the $X^{\prime} X$ matrix. The Box-Draper design in three variables is 


$$
\left[\begin{array}{rrr}
-1 & -1 & -1 \\
1 & -1 & -1 \\
-1 & 1 & -1 \\
-1 & -1 & 1 \\
0.1925 & 0.1925 & -1 \\
0.1925 & -1 & 0.1925 \\
-1 & 0.1925 & 0.1925 \\
-0.2912 & 1 & 1 \\
1 & -0.2912 & 1 \\
1 & 1 & -0.2912
\end{array}\right]
$$

\subsubsection{Computer Generated Minimum Point D-Optimal Designs}

There are several computer algorithms available which search for a set of design points which yield the smallest generalized variance for a specified model. Two of these procedures are DETMAX (Mitchell (1974)) and the branch and bound algorithm due to Welch (1982). Both algorithms require the user to supply a set of candidate design points from which the $N$ points of the design are to be selected.

The three variable designs listed in Table 2.1 were generated by these algorithms. MB10 was generated using DETMAX in a study by Mitchell and Bayne (1978). The other three designs were generated by the branch and bound algorithm described by Welch (1982). In all cases, the set of candidate points was the set of design points of a $3^{3}$ factorial design. Thus, each of the designs represents a fraction of a $3^{3}$ factorial design. 
Table 1.

Design Points for Computer Generated Second Order Designs with Three Factors at Three Levels

\begin{tabular}{cccccccc} 
N & Design * & \multicolumn{7}{c}{ Design Points ** } \\
& & & & & \\
10 & MB10 & 020 & 220 & 110 & 102 & 000 & 211 \\
& & 200 & 012 & 222 & 121 & & \\
10 & WC10 & 002 & 010 & 021 & 101 & 112 & 200 \\
& & 202 & 211 & 220 & 222 & & \\
& & & & & & & \\
10 & WD10 & 000 & 002 & 021 & 101 & 110 & 200 \\
& & 202 & 211 & 220 & 222 & & \\
& & & & & & & \\
11 & WC11 & 000 & 002 & 020 & 022 & 110 & 121 \\
& & 200 & 202 & 211 & 220 & 222 &
\end{tabular}

* The last two digits of the design name indicate the number of design points, $N$.

$\mathrm{MB}=\mathrm{D}$-optimal design generated by DETMAX in Mitchell and Bayne (1978).

WD $=\mathrm{D}$-optimal design generated by the branch and bound algorithm in Welch (1982).

$\mathrm{WC}=\mathrm{A}$ compromise design generated by a modification of the branch and bound algorithm in Welch (1982). This design was selected to achieve the smallest maximum prediction variance at the design points and the smallest average prediction variance over the distinct design points among designs in the class for which $\left|X^{\prime} X\right| \geq 0.95\left|X_{D}^{\prime} X_{D}\right|$, where $\left|X_{D}{ }^{\prime} X_{D}\right|^{-1}$ is the generalized variance for the D-optimal design.

Thus, the D-optimality criterion was used in conjunction with prediction variance criteria.

** The levels are recorded as 0,1 and 2 for convenience. The actual levels of the design variables used are $-1,0$ and 1 respectively. 


\section{Chapter III}

\section{Assessment of Prediction Capability on Spheres}

\subsection{Existing Methods}

Response surface studies are used to improve system output. Often this involves finding the combination of levels of the influential variables which optimizes the response of the system. Improvement would occur if, for example, the yield of the system is maximized or if the deviation of the response from a certain norm is minimized.

Having chosen a design in an appropriate region of the independent variables, the experimenter begins the search for the optimum response in all directions from the center of the region. The behavior of the response at all points in the region which are the same distance from the region center, that is, points on the surface of a k-dimensional hypersphere, will determine the direction of the search. For this reason, the assessment of the quality of the estimated responses on spheres is an essential consideration in the selection of an experimental design. 
Clearly, the proper description of the prediction capability on all spheres in the region for a design could not be accomplished with a single-valued criterion. Indeed, it would require a norm which depends on the radius, $r$, of the $\mathrm{k}$-dimensional hypersphere. The criterion would have a value for each $r$, from the center to the edges of the region. An ideal way to view such a continuum of norms is to plot the values of the criterion against the radius $r$. A comparison of such plots, for competing designs will aid in the selection of a good experimental design.

\subsubsection{The Problem with D-Efficiency}

The D-efficiency or D-optimality criterion for choosing a response surface design has been given considerable attention in the literature, (see St. John and Draper (1975) for a comprehensive bibliography through 1975). It is often recommended to the researcher if only one design criterion is to be considered. Also, many designs have been constructed to have the D-optimality property or to be nearly D-optimal, (see, for example, Box and Draper (1971, 1974), Atkinson (1973), Mitchell and Bayne (1978) and Welch (1982)).

D-efficiency is an assessment of the precision of the coefficients of the estimated response surface model. It has been shown (Kiefer and Wolfowitz (1960)) that for continuous designs the property of D-optimality is equivalent to the prediction variance property of G-optimality. That is, the design which achieves maximum $\left|X^{\prime} X\right|$ among all designs appropriate for the model also achieves the minimum maximum prediction variance in the region. However, there is no connection between the D-efficiency and G-efficiency of a design which is not D-optimal.

Although D-optimality is linked to prediction capability through the equivalence theorem of Kiefer and Wolfowitz (1960), the D-efficiency criterion does not provide an assessment of the quality of prediction on spheres. In particular, it does not provide a graphical representation of the 
prediction variances at locations on spheres. Thus, the D-efficiency criterion is not a suitable measure of prediction capability on spheres.

\subsubsection{Prediction Variance Contours}

Plots of constant prediction variance in pairs of the independent variables is the classical method of viewing prediction variances in the region. In an experiment involving only two independent variables, the contour plot provides a complete picture of the prediction capability of the experimental design. By careful investigation of the contours the researcher can ascertain the nature of the prediction variances at locations which are the same distance from the region center.

As an example, consider fitting a first order model in two variables with a $2^{2}$ factorial design with three experimental runs in the center of the region. Contours of equal prediction variances are plotted in Figure 1. Values for the contours are given by the legend $\mathrm{V}$ below the plot. The variance is evaluated apart from $\sigma^{2}$; that is, the contours are plots of $\operatorname{Var}(\hat{y}(\underline{x})) / \sigma^{2}$. It is clear by the circular nature of the contours that the response is being estimated with equal precision at all locations which are the same distance from the center. That is, the $2^{2}$ factorial design is a rotatable design. Suppose, however, that measurement of the levels of the variables was inaccurate due to a faulty measurement device so that the actual design settings used in the experiment are as follows:

$$
\left[\begin{array}{rr}
0.75 & 0.90 \\
-0.90 & 0.90 \\
0.80 & -0.90 \\
-0.80 & -0.75 \\
0 & 0 \\
0 & 0 \\
0 & 0
\end{array}\right]
$$


From the prediction variance contour plot for this design given in Figure 2 it can be seen that the improper settings hinder the predicting ability of the $2^{2}$ factorial design at nearly all locations in the region. The contours are somewhat elongated indicating that, although there appears to be nearly equal prediction variances on spheres, the design no longer possesses the rotatability property. Also, the prediction capability diminishes more rapidly as one predicts further from the center when there are improper settings. For example, the variance of an estimated response at $x_{1}=1.5$ and $x_{2}=0$ is 0.7 when the readings on the settings are accurate. The actual prediction variance when the readings are not accurate is 1.0 , a sizeable increase.

The use of contour plots in an experiment involving $\mathrm{k}>2$ independent variables requires a plot of prediction variance contours for each pair of variables. The remaining $k-2$ variables are held fixed to form the contours for two variables. The information from each plot must be pieced together to form a picture of the prediction variances for the design. This is often a difficult task. Prediction variance contour plots are rarely used in practice, particularly, when more than three independent variables are present in the system.

\subsubsection{Prediction Variance Pictures for Rotatable Designs}

Recall that a rotatable design, by definition, yields equal variances of the predicted response at all locations which are equidistant from the center of the region of interest. If the design is rotatable, a plot of the prediction variance, $\operatorname{Var}(\hat{y}(\underline{x})) / \sigma^{2}=g\left(r^{2}\right)$, against the radius $r$ provides all the pertinent information about the prediction variances on spheres for the design. Thus, the rotatability property facilitates the investigation of prediction capability on spheres for a design. Comparisons of rotatable designs on a per radius basis could easily be made by comparing plots of their prediction variances as functions of the radius $r$. 

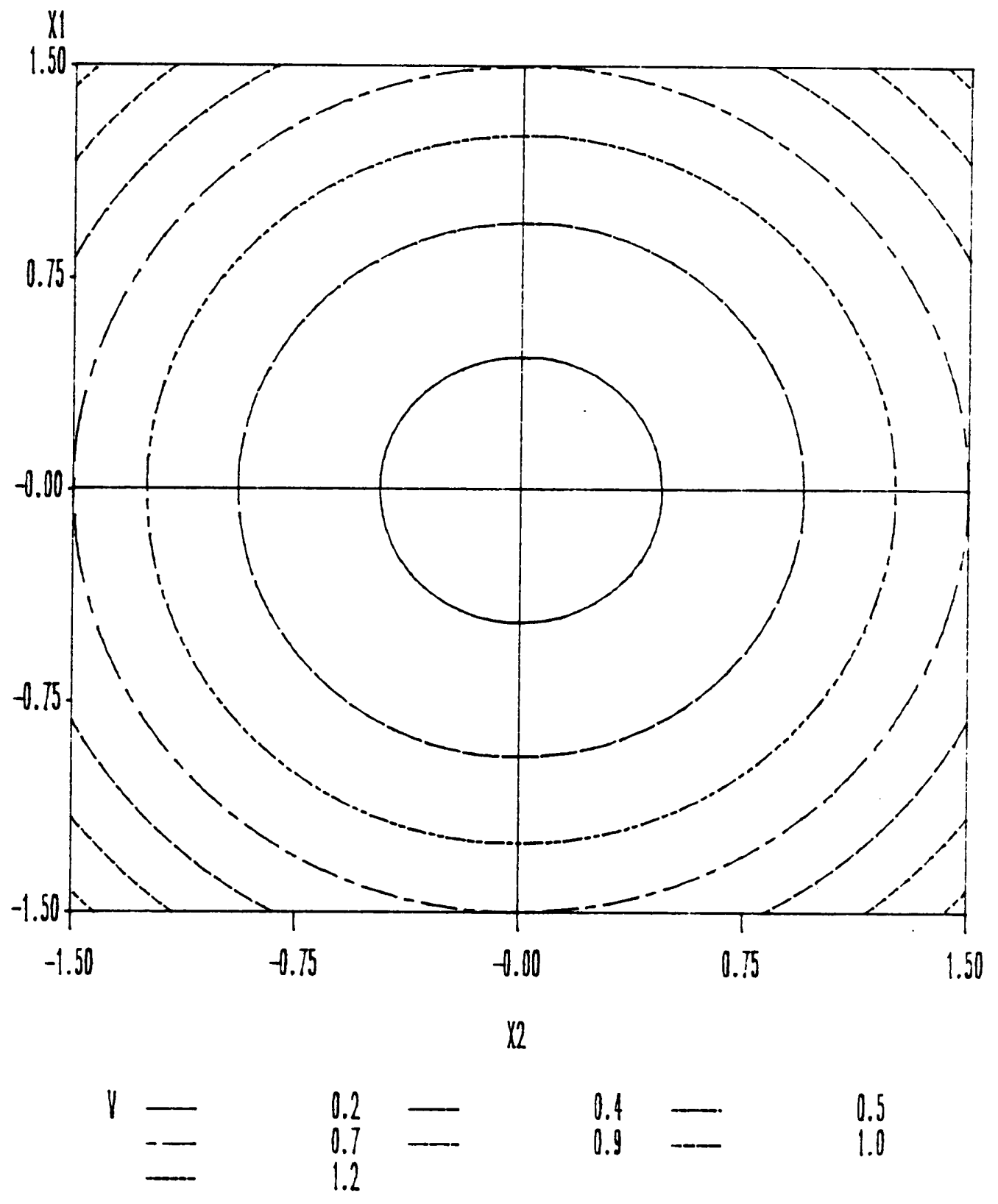

FIGORE 1.

PRDICTION VARIANE CONTORS

OOR A 2-SQURRED FACTORILL DESIGN 

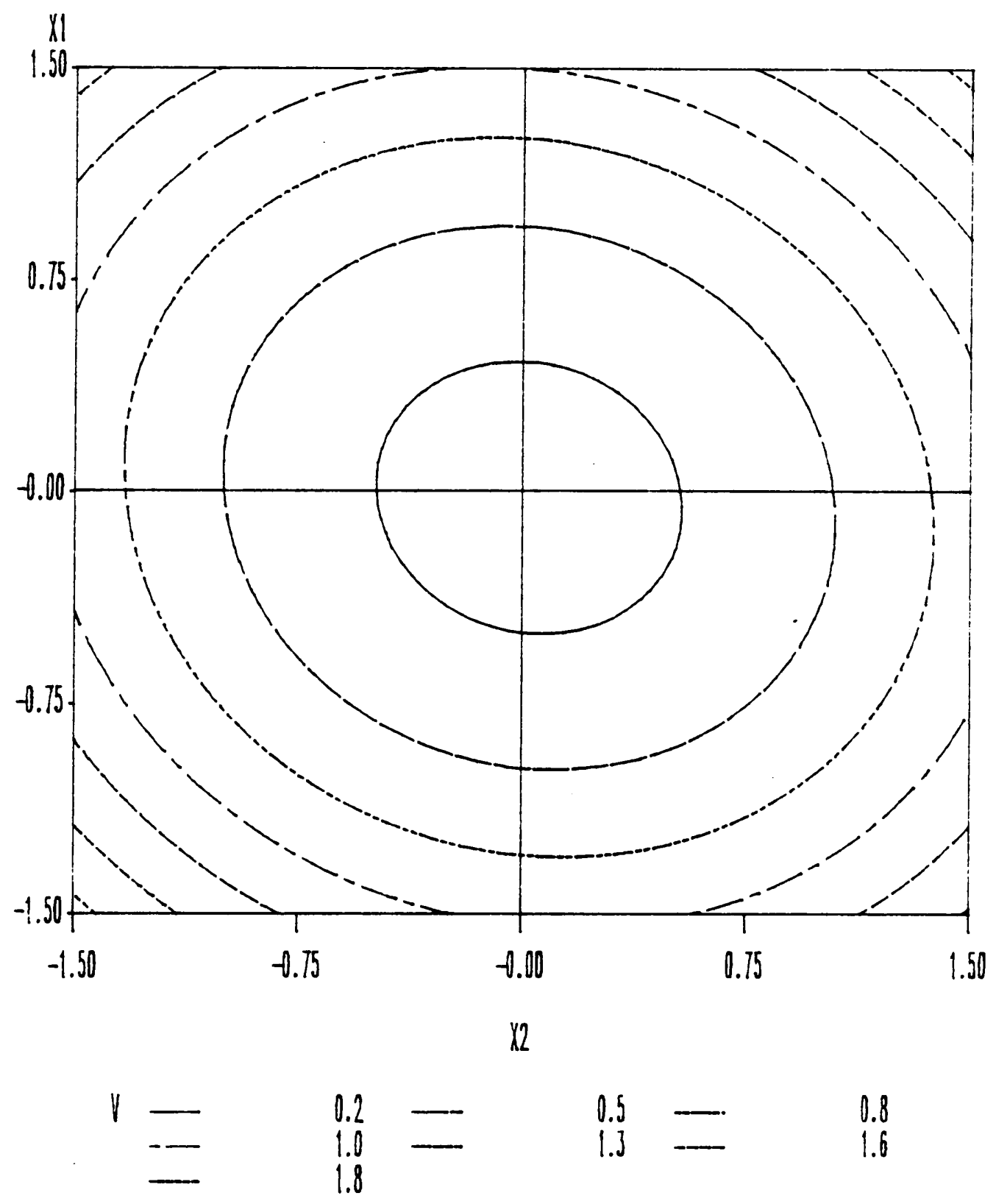

FIOUPR 2.

PREDICTIOI VRRIAME CONTORS

FOR A 2-SPUIRED FACTORILL DESIGI

IIIH IIPPOPER SETTINSS 
The use of plots of $N \operatorname{Var}(\hat{y}(\underline{x})) / \sigma^{2}$ against the distance from the region center to compare rotatable designs has been advocated since RSM was first established. Box and Hunter (1957) suggested choosing a rotatable design for which the prediction variance at the center of the region is equal to the prediction variance at locations on a sphere of radius 1 . The uniform precision property prevents the prediction variance from increasing rapidly as the responses are being estimated further from the region center. The prediction variance plots were used to choose the rotatable design from among a set of rotatable designs which most nearly had the uniform precision property. Such plots have fallen into disuse as a method of viewing the prediction capability of a rotatable design.

The prediction variances for two rotatable designs are compared over a range of radii in Figure 3. The design which generated the curve identified by the solid line (design 1 ) is a $2^{3}$ factorial design. The design corresponding to the second curve (design 2), identified by the dashed line, is a $2^{3}$ factorial design with two additional points in the center of the region. Since there are a different number of design points in each design, $N=8$ versus $N=10$, the prediction variances have been weighted by the sample size, $N$. This has the effect of rewarding the smaller design, design 1 in this example, for being more cost efficient than the larger design. From the plot it is clear that the design without the center runs is more efficient than the larger design. Although the addition of two center points to the $2^{3}$ factorial design naturally results in increased precision in the estimation of the response at all locations, the precision gained is not enough to warrant taking the extra experimental runs. Of course, center runs may be added to a design for other reasons, for example, to gain degrees of freedom for a test of lack of fit of the model.

\subsubsection{Prediction Variance Pictures for Non-Rotatable Designs}

The prediction capability of a design which is not rotatable is more difficult to assess since the variance of a predicted response in this case depends on the exact location, direction and distance 


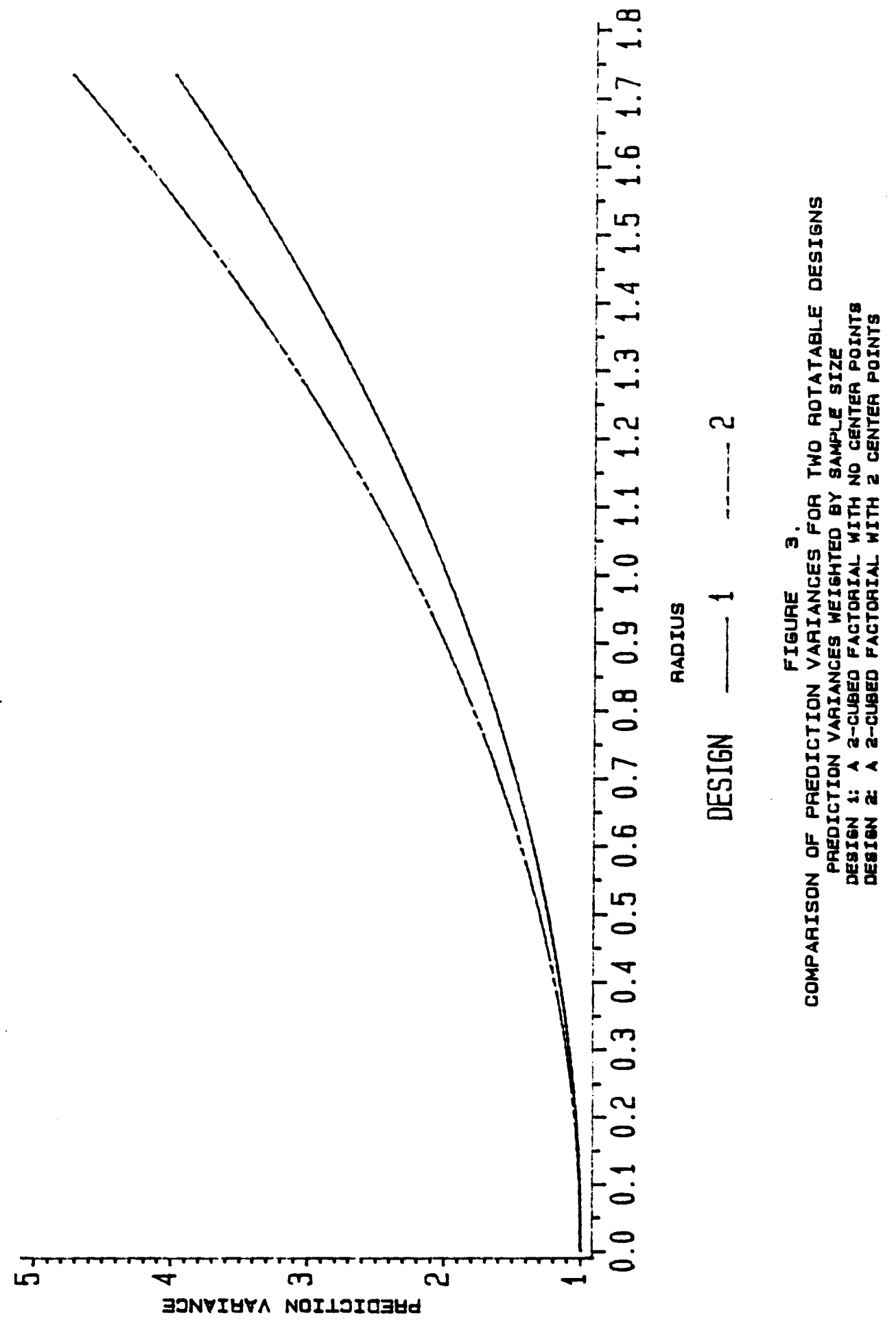


from the center, at which the response is being estimated. The integrated prediction variance for a design provides a single measure of the prediction quality over some region. However, this does not give adequate information about how well the response is being estimated on spheres. There is a need for a norm which is a function of the radius of the hypersphere and which lends itself to graphical representation as in the case of a rotatable design.

To compare the quality of prediction on hyperspheres among non-rotatable designs, or between non-rotatable and rotatable designs, Hussey, Myers and Houck (1987) introduced the notion of averaging the variances of the estimated responses over the surface of a sphere. The spherical variance, $V^{r}$, over the surface of a k-dimensional hypersphere of radius $r$, defined by $U_{r}=\left\{\underline{x}: \sum_{i=1}^{k} x_{i}^{2}=r^{2}\right\}$ for a design $D$ is given by

$$
V^{r}(D)=\frac{\Psi}{\sigma^{2}} \int_{U_{r}} \operatorname{Var}(\hat{y}(\underline{x})) d \underline{x}
$$

where $\Psi^{-1}=\int_{U_{r}} d x$ is the surface area of $U_{r}$.

Note the similarity of the spherical variance and the integrated prediction variance described previously. Indeed, apart from a factor of $N$, spherical variance is a special case of integrated prediction variance when the region under consideration is the surface of a $\mathrm{k}$-dimensional hypersphere of radius $r$. The spherical variance can be plotted against the radius, $r$, to obtain a picture of the average behavior of prediction variances on spheres for a non-rotatable design.

To illustrate the use of the spherical variance to compare designs, consider three designs to fit a first order model in three variables to the response of a system. Suppose the researcher is interested in predicting the response in a hypersphere of radius $\sqrt{3}=1.732$. The center of the sphere is at $\left(\begin{array}{lll}x_{1} & x_{2} & x_{3}\end{array}\right)=\left(\begin{array}{lll}0 & 0 & 0\end{array}\right)$. Let D1 denote the first candidate design, a $2^{3}$ factorial design. 


$$
\text { D1 }=\left[\begin{array}{rrr}
1 & 1 & 1 \\
1 & 1 & -1 \\
1 & -1 & 1 \\
-1 & 1 & 1 \\
1 & -1 & -1 \\
-1 & 1 & -1 \\
-1 & -1 & 1 \\
-1 & -1 & -1
\end{array}\right]
$$

D1 is a rotatable design in the first order model case. The second design, D2, is a $2^{3}$ factorial in which two of the design points have been inadvertantly dropped from the design. D2 is not rotatable.

$$
\mathrm{D} 2=\left[\begin{array}{rrr}
1 & 1 & -1 \\
1 & -1 & 1 \\
-1 & 1 & 1 \\
1 & -1 & -1 \\
-1 & 1 & -1 \\
-1 & -1 & 1
\end{array}\right]
$$

The third design selected for comparison is a non-rotatable three level design with 8 design points given by 


$$
\mathrm{D} 3=\left[\begin{array}{rrr}
1 & 1 & 1 \\
1 & 1 & 0 \\
1 & 0 & 1 \\
0 & 1 & 1 \\
-1 & -1 & 0 \\
-1 & 0 & -1 \\
0 & -1 & -1 \\
-1 & -1 & -1
\end{array}\right]
$$

Observe that each design has center at $\left(\begin{array}{lll}x_{1} & x_{2} & x_{3}\end{array}\right)=\left(\begin{array}{lll}0 & 0 & 0\end{array}\right)$. That is, the design center and center of the region under consideration are the same.

The average prediction variance for each design was computed for 21 values of the radius, $r$, ranging from $r=0$, (representing prediction at the region center), to $r=\sqrt{3}=1.732$, (representing prediction at locations on the perimeter of the region of interest). The results are presented for comparison in Table 2 and in a plot of $V^{r}$ versus $r$ in Figure 4. The spherical variances for each design have not been weighted by the appropriate sample size even though the sample sizes differ for the three designs. The second design, D2, was originally intended to be identical to the $2^{3}$ factorial design. Eight experimental runs were attempted using the D2 design, however, two were either not completed or the information gathered from the two was lost. Thus, it would not be fair to 'reward' the design for a loss of information by weighting the spherical variances by the number of completed design runs. For this reason, the designs were compared as if each consisted of eight design points.

The rotatable design $\mathrm{D} 1$ is the best of the three designs with respect to average prediction variance for all radii. The loss of two design points in D2 resulted in a considerable loss of precision as well. Note that with no additional information in the center, usually provided by center runs, D2 does not predict nearly as well as D1 and D3 in the center. D3, chosen solely for its use in il- 
Table 2.

Spherical Variances for Example Designs of Section 3.1.5

\section{DESIGN *}

$\begin{array}{lccc}\text { RADIUS } & \text { D1 } & \text { D2 } & \text { D3 } \\ & & & \\ 0.0000 & 0.1250 & 0.1667 & 0.1250 \\ 0.0866 & 0.1259 & 0.1685 & 0.1277 \\ 0.1732 & 0.1288 & 0.1742 & 0.1357 \\ 0.2598 & 0.1334 & 0.1835 & 0.1491 \\ 0.3464 & 0.1400 & 0.1967 & 0.1678 \\ 0.4330 & 0.1484 & 0.2135 & 0.1920 \\ 0.5196 & 0.1587 & 0.2342 & 0.2214 \\ 0.6062 & 0.1709 & 0.2585 & 0.2562 \\ 0.6928 & 0.1850 & 0.2867 & 0.2964 \\ 0.7794 & 0.2009 & 0.3185 & 0.3419 \\ 0.8660 & 0.2187 & 0.3542 & 0.3928 \\ 0.9526 & 0.2384 & 0.3935 & 0.4491 \\ 1.0392 & 0.2600 & 0.4366 & 0.5107 \\ 1.1258 & 0.2834 & 0.4835 & 0.5776 \\ 1.2124 & 0.3087 & 0.5341 & 0.6500 \\ 1.2990 & 0.3359 & 0.5885 & 0.7276 \\ 1.3856 & 0.3650 & 0.6466 & 0.8107 \\ 1.4722 & 0.3959 & 0.7085 & 0.8991 \\ 1.5588 & 0.4287 & 0.7741 & 0.9928 \\ 1.6454 & 0.4634 & 0.8435 & 1.0919 \\ 1.7320 & 0.5000 & 0.9166 & 1.1964\end{array}$

* The example designs are first order designs in three variables.

$\mathrm{D} 1=\mathrm{A} 2^{3}$ factorial design, $N=8$.

D2 $=\mathrm{A} 2^{3}$ factorial design with the design points

( 11111$)$ and $(-1-1-1)$ missing, $N=6$.

$\mathrm{D} 3=\mathrm{A}$ 3-level design, $N=8$. 
lustration, has comparable prediction variance to D1 for locations close to the center of the design. However, the prediction quality of D3 quickly diminishes as one predicts further out from the center of the design.

In addition to the average prediction variance, the prediction variance, $\operatorname{Var}(\hat{y}(\underline{x})) / \sigma^{2}$, was calculated for the individual locations given in Table 3 for each design. These locations were selected to represent prediction near the center, $r=0.5$, toward the middle, $r=1$, and on the boundary, $r=1.732$, of the region under consideration. The prediction variances are presented in Table 4. Note that the design D1 is estimating the responses at locations the same distance from the center of the region with equal precision. Designs D2 and, in particular, D3 have noticeably different variances for the predicted responses at equidistant locations from the center of the region. These designs are not providing consistent estimation of the response on spheres. It is apparent from this illustration that the spherical variance does not give a complete picture of the prediction capability on spheres of a non-rotatable design. Measures to fill out the description of prediction capability on spheres will be presented in the next section.

\subsection{Prediction Variance Dispersion Measures}

\subsubsection{The Need for Prediction Variance Dispersion Measures}

The spherical variance provides a view of the prediction variance properties of a non-rotatable design which was not previously available to the researcher. Although the spherical variance measures how well the responses are estimated on the average at locations on a sphere, it does not indicate the consistency with which these responses are estimated. As with any average, the spherical variance requires a measure of how well the average describes the prediction variances as 


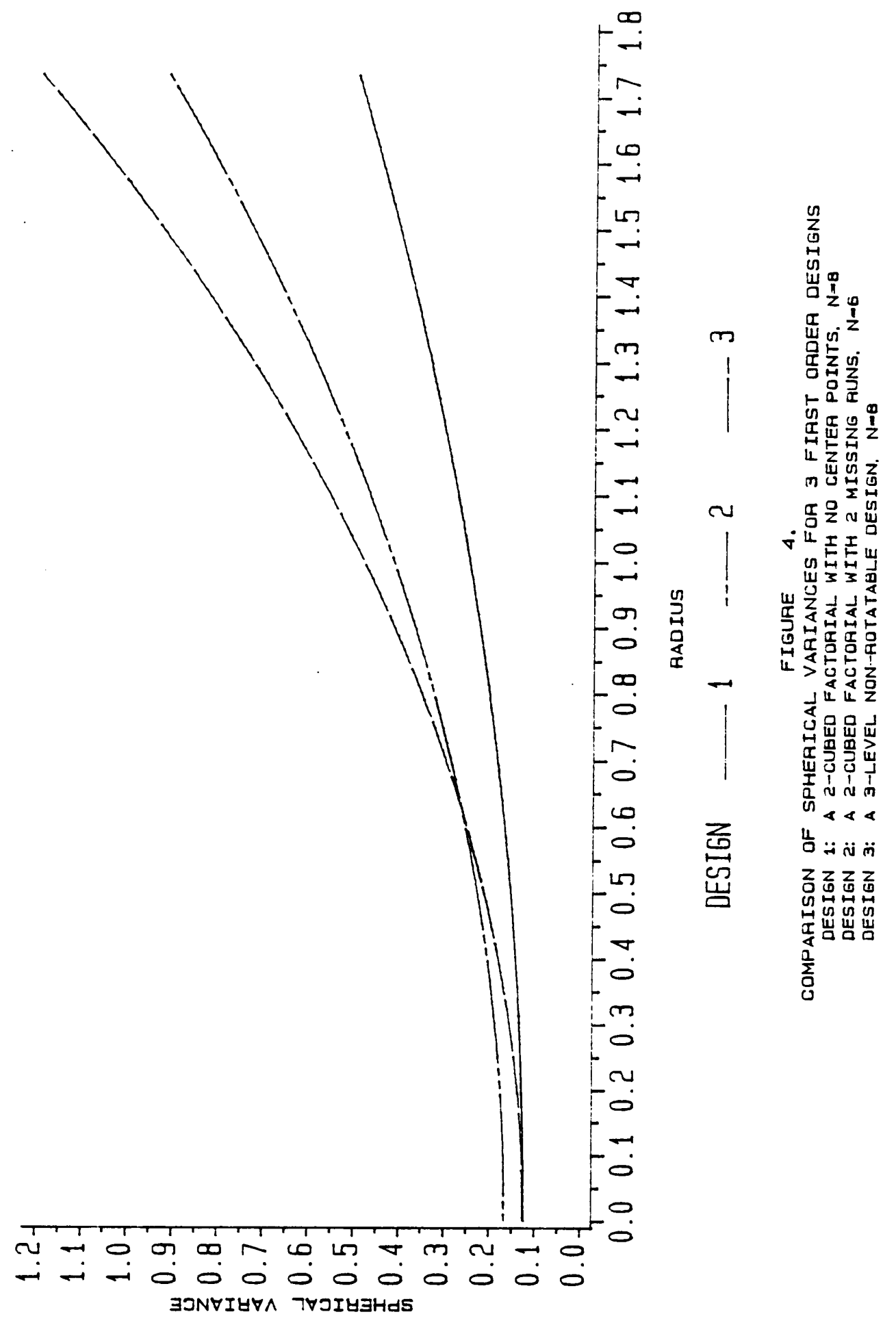


Table 3.

Locations for Calculation of Prediction Variance for Example Designs of Section 2.1.5

\begin{tabular}{lllll} 
RADIUS & & $x_{1}$ & $x_{2}$ & \multicolumn{1}{c}{$x_{3}$} \\
& & & & \\
0.5 & P1 & 0.5 & 0.0 & 0.0 \\
& P2 & 0.2887 & 0.2887 & 0.2886 \\
& P3 & 0.25 & 0.433 & 0.0 \\
1.0 & P4 & 1.0 & & \\
& P5 & 0.577 & 0.0 & 0.0 \\
& P6 & 0.456 & 0.89 & 0.578 \\
1.732 & P7 & 1.732 & 0.0 & 0.0 \\
& P8 & 1.0 & 1.0 & 0.0 \\
& P9 & 1.5 & 0.865 & 1.0 \\
& & & & 0.0
\end{tabular}

Table 4.

Prediction Variances for the Locations Given in Table 3.

\section{DESIGN *}

\begin{tabular}{lcccc} 
RADIUS & LOCATION & D1 & D2 & D3 \\
\cline { 2 - 4 } & & & & \\
& P1 & 0.15625 & 0.22916 & 0.21429 \\
& P2 & 0.15625 & 0.29166 & 0.14286 \\
1.0 & P3 & 0.15625 & 0.25623 & 0.18335 \\
& & & & \\
& P4 & 0.25 & 0.41667 & 0.48214 \\
1.732 & P5 & 0.25 & 0.66664 & 0.19642 \\
& P6 & 0.25 & 0.51814 & 0.36620 \\
& P7 & 0.5 & 0.91662 & 1.19637 \\
& P8 & 0.5 & 1.66667 & 0.33929 \\
& P9 & 0.5 & 1.24060 & 0.82508
\end{tabular}

- The example designs are first order designs in three variables.

$\mathrm{D} 1=\mathrm{A} 2^{3}$ factorial design, $N=8$.

$\mathrm{D} 2=\mathrm{A} 2^{3}$ factorial design with the design points

( 1111 ) and ( $-1-1-1)$ missing, $N=6$.

$\mathrm{D} 3=\mathrm{A}$ 3-level design, $N=8$.

Variances are $\operatorname{Var}(\hat{y}(\underline{x})) / \sigma^{2}$ where the locations $\underline{x}=\left(\begin{array}{lll}x_{1} & x_{2} & x_{3}\end{array}\right)^{\prime}$ are given in Table 3 . 
a whole. To judge how well the spherical variance represents the prediction capability of a design, two measures which describe the dispersion in the variances of the predicted responses on a sphere will be developed in this section. These prediction variance dispersion (PVD) measures will be based on the usual ideas of variability.

There is no variability in the variances of the predicted responses on a sphere for a rotatable design. Dispersion in the variances for a non-rotatable design indicates a deviation from rotatability. Hence, the magnitude of the dispersion measure corresponding to a sphere for a design gauges how near that design is to possessing the rotatability property of equal prediction variances on the sphere.

The indices proposed by Khuri (1988) and by Draper and Guttman (1988), and discussed in section 2.3 .4 , also attempt to quantify how near a design is to possessing the rotatability property. However, each is a single-valued measure which describes the 'nearness to rotatability' of the design as a whole and does not address the consistency of prediction on spheres. The PVD measures, on the other hand, describe the consistency with which the response is estimated on a particular sphere. Consideration of these measures across a range of radii will allow the researcher to ascertain at what distances from the region center the design is estimating the response with nearly equal precision and where the design is providing estimates with inconsistent accuracy.

\subsubsection{Measuring the Dispersion in Prediction Variances}

If we view each location $x$ on the surface of the k-dimensional hypersphere defined by $U$, as a possible value of a vector-valued random variable, say $\mathrm{X}$, then the spherical variance, $\mathrm{Vr}$, is analogous to the mean or expected value of $\operatorname{Var}(\hat{y}(\mathrm{X})) / \sigma^{2}$. Note that the factor $\Psi=\left[\int_{v_{r}} d x\right]^{-1}$ in the definition of $V^{r}$ plays the role of the probability density function of $\mathrm{X}$. Thus, the random 
variable $\mathrm{X}$ is assumed to be uniformly distributed over the surface of the hypersphere defined by $U_{r}$.

Consider, now, measures of the variability in $\operatorname{Var}(\hat{y}(\mathrm{X})) / \sigma^{2}$ where $\mathrm{X}$ takes values in $U_{r}$. The simplest dispersion measure is the range of the values. A range for $\operatorname{Var}(\hat{y}(\mathrm{X})) / \sigma^{2}$ is

$$
\operatorname{Rof} V(r)=\max _{\underline{x} \in U_{r}} \operatorname{Var}(\hat{y}(\underline{x})) / \sigma^{2}-\min _{\underline{x} \in U_{r}} \operatorname{Var}(\hat{y}(\underline{x})) / \sigma^{2}
$$

Perhaps the most conventional measures of dispersion are the variance and standard deviation. The variance of a random variable is the expected value of the squared deviation from the mean. Thus, the random variable $\operatorname{Var}(\hat{y}(\mathrm{X})) / \sigma^{2}$ with mean $V^{r}$ has variance given by

$$
\operatorname{Vof} V(r)=\Psi \int_{U_{r}}\left[\frac{1}{\sigma^{2}} \operatorname{Var}(\hat{y}(\underline{x}))-V^{r}\right]^{2} d \underline{x}
$$

As before, $\Psi$ represents the probability density function of $\mathrm{X}$.

Since the form of $\operatorname{Var}(\hat{y}(\underline{x}))$ depends on the chosen model, the forms of the range and variance will also depend on the model. Further results for the spherical variance, RofV and VofV measures will be derived when the assumed model of the response is first order. In addition, the form of the VofV will be extended to the case of fitting a second order model in two independent variables to the response. An optimization algorithm will be implemented to find the RofV in the second order case. A general form of the spherical variance due to Hussey, Myers and Houck (1987) will also be given.

\subsubsection{Some Notation}

Before proceeding, we shall set forth some notation which will be used in developing rangeand variance-type measures as discussed previously. The prediction variance dispersion measures 
and spherical variance will be investigated further under three experimental situations. Modelspecific forms will be derived under a first order model for the response in case I and case II. The experimental situation in which the design is centered about the origin of the region of interest will be considered first. The more complicated case in which the design center and region center differ will be investigated in case II. The evaluation of these measures in the second order model case will be discussed under case III.

\section{Case I : A first order model; the design center and region center are the same.}

Suppose a first order model in $\mathrm{k}$ variables is fit to the response with a design centered about the center of the region of the independent variables. This is the region of interest. Let $x=\left(\begin{array}{lllll}x_{1} & x_{2} & \ldots & x_{k}\end{array}\right)^{\prime}$ be a setting of the levels of the variables $x_{1}, x_{2}, \ldots, x_{k}$ present in the system and write $\underline{x}^{*}=(1 \underline{x})^{\prime}$. Define the $N \mathrm{x}(\mathrm{k}+1)$ matrix $X^{\circ}$ by $[1 X]$ where 1 is an $N \mathrm{x} 1$ vector of ones and $X$ is an $N$ xk matrix whose columns are the last $\mathrm{k}$ columns of the matrix defined by

(2.2.2) for a first order model. $X$ is the model-matrix. The first order model can be written as

$$
\underline{y}=X^{*} \underline{\underline{L}}+\underline{\varepsilon}
$$

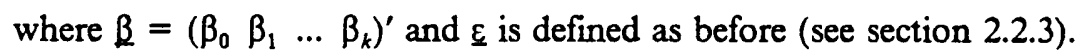

In the present discussion, the columns of the submatrix $X$ are centered so that $\sum_{i=1}^{N} x_{i j}=0$ for all $\mathrm{j}=1,2, \ldots, \mathrm{k}$. In this way, the vector of ones in $X$ is orthogonal to the submatrix $X$. This assumption allows us to write the $\left(X^{*} X^{*}\right)$ and $\left(X^{*} X^{*}\right)^{-1}$ matrices in the following convenient forms

$$
X^{* \prime} X^{*}=\left[\begin{array}{cc}
N & Q^{\prime} \\
0 & X^{\prime} X
\end{array}\right]
$$

and 


$$
\left(X^{* \prime} X^{*}\right)^{-1}=\left[\begin{array}{cc}
\frac{1}{N} & \underline{Q}^{\prime} \\
\underline{0} & \left(X^{\prime} X\right)^{-1}
\end{array}\right] .
$$

Consider the eigenvalue decomposition of $\left(X^{\prime} X\right)^{-1}$. Define $P$ to be the kxk orthogonal matrix for which

$$
P^{\prime}\left(X^{\prime} X\right)^{-1} P=\Lambda
$$

where $\Lambda=\operatorname{diagonal}\left(\lambda_{1}, \lambda_{2}, \ldots, \lambda_{k}\right)$ is the diagonal matrix containing the eigenvalues of $\left(X^{\prime} X\right)^{-1}$. Since $P$ is an orthogonal matrix we have that $P^{\prime} P=P P^{\prime}=I$ the identity matrix. Consequently, the variance of a predicted response for a first order model can be written as

$$
\begin{aligned}
\operatorname{Var}(\hat{y}(\underline{x})) & =\sigma^{2}\left[\frac{1}{N}+\underline{x}^{\prime} P P^{\prime}\left(X^{\prime} X\right)^{-1} P P^{\prime} \underline{x}\right] \\
& =\sigma^{2}\left[\frac{1}{N}+z^{\prime} \Lambda z\right] \\
& =\sigma^{2}\left[\frac{1}{N}+\sum_{i=1}^{k} \lambda_{i} z_{i}^{2}\right]
\end{aligned}
$$

where $z=\left(\begin{array}{lllll}z_{1} & z_{2} & \ldots & z_{k}\end{array}\right)^{\prime}=P^{\prime} \underline{x} . \quad P$ is an orthogonal transformation taking $\underline{x}$ into $z$. Note that $\sum_{i=1}^{k} z_{i}^{2}=z^{\prime} \underline{z}=\underline{x}^{\prime} \underline{x}=\sum_{i=1}^{k} x_{i}^{2}$. Thus, for any point $\underline{x}$ located on $U_{r}$, the surface of a k-dimensional hypersphere of radius $r$, the corresponding point after orthogonal transformation by $P$, that is, $\underline{z}=P^{\prime} \underline{x}$, is also on $U_{r}$.

Case II : A first order model; the design center and region center are not the same.

The case of centered columns of the submatrix $X$ for a first order model corresponds to the origin of the design and the origin of the region of the independent variables being the same. The center of the design, however, is not always identical to the center of the region of interest. In general, define the location of the center of the design to be $\underline{h}=\left(\begin{array}{llll}h_{1} & h_{2} & \ldots & h_{k}\end{array}\right)^{\prime}$; the center of the region is $\underline{0}=\left(\begin{array}{llll}0 & 0 & \ldots & 0\end{array}\right)^{\prime}$ in the design variables. When $\underline{h} \neq \underline{0}$ the columns of the submatrix $X$ are not centered. It will prove useful to consider a translation of the axis system corresponding 
to the design variables $x_{1}, x_{2}, \ldots, x_{k}$ to an axis system with origin at the center of the design. That is, the point $\underline{h}$ in the $x$-axis system is the point $\underline{0}$ in the new axis system. Call the variables in the new axis system $w_{1}, w_{2}, \ldots, w_{k}$, where $w_{i}=x_{i}-h_{i}, i=1,2, \ldots, k$.

In terms of the $w$-variables, the variance of a predicted response at a point $\underline{w}=\left(\begin{array}{llll}w_{1} & w_{2} & \ldots & w_{k}\end{array}\right)^{\prime}=\left(\begin{array}{llll}x_{1}-h_{1} & x_{2}-h_{2} & \ldots & x_{k}-h_{k}\end{array}\right)^{\prime}=\underline{x}-\underline{h}$ is given by

$$
\operatorname{Var}(\hat{y}(\underline{w}))=\sigma^{2} \underline{w}^{* \prime}\left(W^{* \prime} W^{*}\right)^{-1} \underline{w}^{*}
$$

where $\underline{w}^{*}=(1 \underline{w})^{\prime}$, and $W^{*}=[1 W]$. The Nxk submatrix $W$ is analogous to the submatrix $X$ in case I. In this case, the columns of $W$ are centered versions of the corresponding columns of $X$. Using the development of case I for a prediction variance at a location in the $w$-axis system,

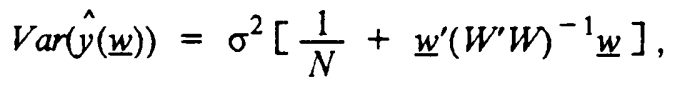

which can be written in terms of the original design variables as

$$
\begin{aligned}
\operatorname{Var}(\hat{y}(\underline{w})) & =\sigma^{2}\left[\frac{1}{N}+(\underline{x}-\underline{h})^{\prime}\left(W^{\prime} W\right)^{-1}(\underline{x}-\underline{h})\right] \\
& =\sigma^{2}\left[\frac{1}{N}+\underline{x}^{\prime}\left(W^{\prime} W\right)^{-1} \underline{x}-2 \underline{x}^{\prime}\left(W^{\prime} W\right)^{-1} \underline{h}+\underline{h}^{\prime}\left(W^{\prime} W\right)^{-1} \underline{h}\right]
\end{aligned}
$$

It will be convenient to express the prediction variance in terms of the eigenvalues of the $\left(W^{\prime} W\right)^{-1}$ matrix. Let $P$ be the kxk orthogonal matrix of the eigenvalue decomposition of $\left(W^{\prime} W\right)^{-1}$. Then,

$$
\begin{aligned}
\operatorname{Var}(\hat{y}(\underline{w})) & =\sigma^{2}\left[\frac{1}{N}+z^{\prime} \Lambda z-2 z^{\prime} \Lambda m+m^{\prime} \Lambda m\right] \\
& =\sigma^{2}\left[\frac{1}{N}+\sum_{i=1}^{k} \lambda_{l} z_{l}^{2}-2 \sum_{i=1}^{k} \lambda_{i} m_{i} z_{i}+\sum_{i=1}^{k} \lambda_{i} m_{i}^{2}\right]
\end{aligned}
$$


where $\underline{z}=\left(\begin{array}{llll}z_{1} & z_{2} & \ldots & z_{k}\end{array}\right)^{\prime}=P^{\prime} \underline{x}$ and $\underline{m}=\left(\begin{array}{llll}m_{1} & m_{2} & \ldots & m_{k}\end{array}\right)^{\prime}=P^{\prime} \underline{h}$.

$\Lambda=\operatorname{diagonal}\left(\lambda_{1}, \lambda_{2}, \ldots, \lambda_{k}\right)$ is the diagonal matrix containing the eigenvalues of $\left(W^{\prime} W\right)^{-1}$. Note that if the design is centered about the origin of the region in the design variables, that is, if $\underline{h}=\underline{Q}$, this formulation reduces, as it should, to the expression given in (3.2.3).

\section{Case III : A second order model.}

Suppose now that a second order model is used to approximate the response surface. In this case, define $\underline{x}^{*}=\left(\begin{array}{lllllllllll}1 & x_{1} & \ldots & x_{k} & x_{1}^{2} & \ldots & x_{k}^{2} & x_{1} x_{2} & x_{1} x_{3} & \ldots & x_{k-1} x_{k}\end{array}\right)^{\prime}$ and the model-matrix

$$
X=\left[\begin{array}{ccccccccccc}
1 & x_{11} & \ldots & x_{1 k} & x_{11}^{2} & \ldots & x_{1 k}^{2} & x_{11} x_{12} & x_{11} x_{13} & \ldots & x_{1, k-1} x_{1 k} \\
1 & x_{21} & \ldots & x_{2 k} & x_{21}^{2} & \ldots & x_{2 k}^{2} & x_{21} x_{22} & x_{21} x_{23} & \ldots & x_{2, k-1} x_{2 k} \\
\cdot & . & . & . & . & . & . & . & . & . & \\
\cdot & . & . & . & . & . & . & . & . & . & \\
\cdot & . & . & . & . & . & . & . & . & . & \\
1 & x_{N 1} & \ldots & x_{N k} & x_{N 1}^{2} & \ldots & x_{N k}^{2} & x_{N 1} x_{N 2} & x_{N 1} x_{N 3} & \ldots & x_{N, k-1} x_{N k}
\end{array}\right] .
$$

The variance of a predicted response estimated at a location $\underline{x}=\left(\begin{array}{llll}x_{1} & x_{2} & \ldots & x_{k}\end{array}\right)^{\prime}$ in the region of the design variables is

$$
\operatorname{Var}(\hat{y}(\underline{x}))=\sigma^{2}\left[\underline{x}^{* \prime}\left(X^{\prime} X\right)^{-1} \underline{x}^{*}\right]
$$

The formulation of the prediction variance used in the first order model case cannot be extended to this case due to the curvature present in the second order model.

\section{Spherical region moments.}

The notion of spherical region moments will be useful in the development of a model-specific form for the spherical variance and for the variance of $\operatorname{Var}(\hat{y}(\underline{x})) / \sigma^{2}$ for locations on the surface of 
a hypersphere defined by $U_{r}=\left\{\underline{x}: \sum_{i=1}^{k} x_{i}^{2}=r^{2}\right\}$. A spherical region moment of order $\delta$ is defined to be

$$
\sigma_{\delta_{1} \delta_{2} \ldots \delta_{k}}=\Psi \int_{U_{r}} x_{1}^{\delta_{1}} x_{2}^{\delta_{2}} \ldots x_{k}^{\delta_{k}} d \underline{x}
$$

where $\Psi^{-1}=\int_{U_{r}} d \underline{x}$ is the surface area of $U$, and $\sum_{i=1}^{k} \delta_{i}=\delta$.

If any $\delta_{l}$ is 0 that subscript is dropped from the designation of the moment. Since $U_{r}$ is a symmetric region, the spherical moment $\sigma_{\delta_{1} \delta_{2} \ldots \delta_{k}}$ is zero whenever any $\delta_{i}$ is odd.

The spherical region moments which will be utilized in the development for the first order model case are second order spherical moments given by

$$
\sigma_{2}=\Psi \int_{U_{r}} x_{i}^{2} d \underline{x}=\frac{r^{2}}{k}
$$

and the fourth spherical moments

$$
\sigma_{4}=\Psi \int_{U_{r}} x_{i}^{4} d x=\frac{3 r^{4}}{k(k+2)}
$$

and

$$
\sigma_{22}=\Psi \int_{U_{r}} x_{i}^{2} x_{j}^{2} d \underline{x}=\frac{r^{4}}{k(k+2)}
$$

In addition to the second and fourth order moments, spherical moments of order six and eight will also be required when the assumed model is second order. The sixth order spherical moments are

$$
\sigma_{42}=\Psi \int_{U_{r}} x_{i}^{4} x_{j}^{2} d \underline{x}=\frac{3 r^{6}}{2(k+4)(k+2)}
$$

and 


$$
\sigma_{6}=\Psi \int_{U_{r}} x_{i}^{6} d \underline{x}=\frac{15 r^{6}}{2(k+4)(k+2)}
$$

and the eighth order spherical moments are

$$
\begin{gathered}
\sigma_{44}=\Psi \int_{U_{r}} x_{i}^{4} x_{j}^{4} d \underline{x}=\frac{9 r^{8}}{2(k+6)(k+4)(k+2)}, \\
\sigma_{62}=\Psi \int_{U_{r}} x_{i}^{6} x_{j}^{2} d \underline{x}=\frac{15 r^{8}}{2(k+6)(k+4)(k+2)} \\
\sigma_{8}=\Psi \int_{U_{r}} x_{i}^{8} d \underline{x}=\frac{105 r^{8}}{2(k+6)(k+4)(k+2)}
\end{gathered}
$$

Here $\mathrm{k}$ is the number of variables in the multiple integral, which, for our purposes, is the number of factors in the system. See Stroud (1971) for details of the integration.

Note that the spherical region moments do not depend in any way on the experimental design or form of the model used in the analysis. Region moments, as the name suggests, only depend on the region of interest. In the case of spherical moments, they are functions of the radius, $r$, and dimension, $\mathrm{k}$, of the hypersphere under consideration.

Define the spherical region moment matrix $S$ by

$$
S=\Psi \int_{U_{r}} \underline{x}^{*} \underline{x}^{* \prime} d \underline{x}
$$

where the structure of $\underline{x}^{*}$ depends on the model in use. In particular, $\underline{x}^{*}$ has been defined in this section for first and second order models. The matrix $S$ contains the spherical region moments pertinent to a specific model. 


\subsubsection{The Range of Variances on the Surface of a Hypersphere}

The general form of the range of variances of the predicted responses on the surface of a hypersphere of radius $r$ defined by $U$, is given by

$$
\left.\operatorname{Rof} V(r)=\max _{\underline{x} \in U_{r}} \operatorname{Var}(\hat{y}(\underline{x})) / \sigma^{2}-\min _{\underline{x} \in U_{r}} \operatorname{Var} \hat{y}(\underline{x})\right) / \sigma^{2}
$$

More specific forms of the range will be discussed under the three cases described in the preceding section.

Case I : A first order model; the design center and region center are the same.

To find a specific form for RofV first consider the maximum and minimum values that the prediction variance can achieve in this situation. Specifically, it is necessary to find the optimum values for $\operatorname{Var}(\hat{y}(\underline{x})) / \sigma^{2}$ when $\underline{x}$ is in $U_{r}$. From the preceding section, this is equivalent to optimizing the function

$$
\frac{1}{\sigma^{2}} \operatorname{Var}(\hat{y}(\underline{x}))=\frac{1}{N}+\lambda_{1} z_{1}^{2}+\lambda_{2} z_{2}^{2}+\ldots+\lambda_{k} z_{k}^{2}
$$

when $\underline{x}=\left(\begin{array}{lllll}x_{1} & x_{2} & \ldots & x_{k}\end{array}\right)^{\prime}$ and, therefore, $\underline{z}=\left(\begin{array}{llll}z_{1} & z_{2} & \ldots & z_{k}\end{array}\right)^{\prime}$ are in $U_{r}$. The coefficients, $\lambda_{1}, \ldots \lambda_{k}$, of the variables in the function are the eigenvalues of the full rank matrix $\left(X^{\prime} X\right)^{-1}$ and, hence, positive. For convenience, suppose that the variables are ordered in such a way that $0<\lambda_{1} \leq \lambda_{2} \leq \ldots \leq \lambda_{k}$.

First consider the case where the eigenvalues are all distinct, that is, $0<\lambda_{1}<\lambda_{2}<\ldots<\lambda_{k}$. To maximize (3.2.7) subject to the condition that $\sum_{i=1}^{k} z_{i}^{2}=r^{2}$ requires that the variable whose coefficient is the largest; i.e., the variable that has the highest weight, be as large as possible. Thus, for the first order case, the maximum variance of prediction on $U_{r}$ occurs for $z_{k}= \pm r$ and $z_{1}=z_{2}=\ldots=z_{k-1}=0$ to achieve $\sum_{i=1}^{k} z_{i}^{2}=r^{2}$. The corresponding maximum is 


$$
\begin{aligned}
\max _{\underline{x} \in U_{r}} \frac{1}{\sigma^{2}} \operatorname{Var}(\hat{y}(\underline{x})) & =\max _{\underline{z} \in U_{r}}\left[\frac{1}{N}+\sum_{i=1}^{k} \lambda_{i} z_{i}^{2}\right] \\
& =\frac{1}{N}+\lambda_{k} r^{2}
\end{aligned}
$$

where $\lambda_{k}$ is the largest eigenvalue of $\left(X^{\prime} X\right)^{-1}$.

Similarly, to find the minimum prediction variance on $U_{r}$, place all the mass allotted to $z_{1}, z_{2}, \ldots, z_{k}$ by the condition $\sum_{i=1}^{k} z_{i}^{2}=r^{2}$ on the variable whose coefficient is the smallest. Now the minimum occurs for $z_{1}= \pm r$ and $z_{2}=z_{3}=\ldots=z_{k}=0$ to achieve $\sum_{i=1}^{k} z_{i}^{2}=r^{2}$. The minimum prediction variance on $U_{r}$ is then

$$
\begin{aligned}
\min _{\underline{x} \in U_{r}} \frac{1}{\sigma^{2}} \operatorname{Var}(\hat{y}(\underline{x})) & =\min _{\underline{z} \in U_{r}}\left[\frac{1}{N}+\sum_{i=1}^{k} \lambda_{i} z_{i}^{2}\right] \\
& =\frac{1}{N}+\lambda_{1} r^{2}
\end{aligned}
$$

where $\lambda_{1}$ is the smallest eigenvalue of $\left(X^{\prime} X\right)^{-1}$. A more rigorous proof of these results appears in Appendix A.1 for the special case of $k=2$ variables.

When two or more of the eigenvalues are the same, the maximum and minimum values may occur at more than one point on $U_{r}$. The resulting optimal values, however, remain the same. As an illustration, consider $\lambda_{1}=\lambda_{2}$ and all other eigenvalues distinct. Clearly, the maximum prediction variance at locations on $U_{r}$ is as before. The minimum, however, may occur at any point on $U_{r}$ for which $z_{1}^{2}+z_{2}^{2}=r^{2}$ and, hence, $z_{3}=\ldots=z_{k}=0$. The corresponding minimum value though is $1 / N+\lambda_{1} r^{2}$ where $\lambda_{1}=\lambda_{2}$ is the smallest eigenvalue of $\left(X^{\prime} X\right)^{-1}$. So the optimum values of the prediction variance on $U_{r}$ are not effected by multiplicities in the eigenvalues of $\left(X^{\prime} X\right)^{-1}$.

It is of interest to note that if all the eigenvalues of $\left(X^{\prime} X\right)^{-1}$ are the same then the prediction variance reduces to a function of $\sum_{i=1}^{k} z_{i}^{2}$, the distance from the center of the design, alone. This means that the responses at every location on the surface of a hypersphere of radius $r$ are estimated 
with the same precision. Hence, a design for which the eigenvalues of $\left(X^{\prime} X\right)^{-1}$ are all equal is a rotatable design in the first order case.

Ultimately, the range of variances of predicted responses on the surface of a hypersphere of radius $r$ for a first order model is

$$
\begin{aligned}
\operatorname{Rof} V(r) & =\left[\frac{1}{N}+\lambda_{k} r^{2}\right]-\left[\frac{1}{N}+\lambda_{1} r^{2}\right] \\
& =r^{2}\left(\lambda_{k}-\lambda_{1}\right)
\end{aligned}
$$

where $\lambda_{k}$ and $\lambda_{1}$ are the largest and smallest eigenvalues of $\left(X^{\prime} X\right)^{-1}$ respectively.

Clearly, the range of prediction variances at locations on any hypersphere of radius $r$ will be zero for a first order rotatable design. For non-rotatable designs, Rof $\mathrm{V}$ is an increasing function of the radius $r$. Thus, the ability of a non-rotatable design to predict nearly equally well at all locations on the surface of a hypersphere of radius $r$ diminishes as one predicts the response further from the center of the region under consideration. The consistency of the quality of prediction for a given $r$ depends on the closeness of the eigenvalues of $\left(X^{\prime} X\right)^{-1}$ through the range $\lambda_{k}-\lambda_{1}$.

\section{Case II : A first order model; the design center and region center are not the same.}

Under these circumstances, the problem of optimizing $\operatorname{Var}(\hat{y}(\underline{x})) / \sigma^{2}$ subject to $\underline{x}=\left(\begin{array}{llll}x_{1} & x_{2} & \ldots & x_{k}\end{array}\right)^{\prime}$ being on the surface of a hypersphere of radius $r$ centered at $\underline{x}=\underline{0}$ is equivalent to the constrained optimization of $\operatorname{Var}(\hat{y}(\underline{w})) / \sigma^{2}$ in the $w$-variables. In terms of the $w$-axis system, the constraint requires that the point $\underline{w}=\left(\begin{array}{llll}w_{1} & w_{2} & \ldots & w_{k}\end{array}\right)^{\prime}$ lie on the surface of a hypersphere of radius $r$ with center at $\underline{w}=\underline{Q}-\underline{h}$.

Using the method of Lagrangian multipliers for finding the stationary points of a function, the maximum and minimum values are found by solving the following set of simultaneous equations: 


$$
\begin{aligned}
& \frac{\partial Q}{\partial w_{1}}=0, \quad \frac{\partial Q}{\partial w_{2}}=0, \quad \ldots \quad \frac{\partial Q}{\partial w_{k}}=0, \quad \frac{\partial Q}{\partial \mu}=0 \\
& \text { where } Q=\frac{1}{\sigma^{2}} \operatorname{Var}(\hat{y}(\underline{w}))-\mu\left[\sum_{i=1}^{k}\left(w_{i}+h_{i}\right)^{2}-r^{2}\right] \\
& =\frac{1}{N}+\underline{w}^{\prime}\left(W^{\prime} W\right)^{-1} \underline{w}-\mu\left[(\underline{w}+\underline{h})^{\prime}(\underline{w}+\underline{h})-r^{2}\right] \text {, }
\end{aligned}
$$

and $\mu$ is the Lagrangian multiplier. The solution to this problem has been worked out in detail in Appendix A.2.

A stationary point of the system defined by (3.2.9), expressed in the design variables, has the form

$$
\underline{x}_{0}=P\left(\frac{\lambda_{1}}{\lambda_{1}-\mu} m_{1} \frac{\lambda_{2}}{\lambda_{2}-\mu} m_{2} \quad \cdots \frac{\lambda_{k}}{\lambda_{k}-\mu} m_{k}\right)^{\prime}
$$

where $P$ is the orthogonal matrix for which $P^{\prime}\left(W^{\prime} W\right)^{-1} P=\Lambda=\operatorname{diagonal}\left(\lambda_{1} \lambda_{2} \quad \ldots \quad \lambda_{k}\right)$ and $m_{i}$ is the $\mathrm{i}$-th element of the vector $m=P^{\prime} h$. The value of $\mu$ determines the status of the stationary point. In particular, the stationary point is a location of maximum prediction variance on a sphere of radius $r$ if $\mu>\lambda_{k}$, the largest eigenvalue of $\left(W^{\prime} W\right)^{-1}$, and $\sum_{i=1}^{k}\left(\frac{\lambda_{i}}{\lambda_{i}-\mu}\right)^{2} m_{i}^{2}=r^{2}$. The stationary point is a minimum on the sphere if $\mu<\lambda_{1}$, the smallest eigenvalue of $\left(W^{\prime} W\right)^{-1}$, and $\sum_{i=1}^{k}\left(\frac{\lambda_{i}}{\lambda_{i}-\mu}\right)^{2} m_{i}^{2}=r^{2}$. In both cases, the second condition on $\mu$ guarantees the the stationary point will be on the surface of the hypersphere of radius $r$.

The relationship between the radius $r$ and the Lagrangian multiplier $\mu$ was established by Draper (1963) in connection with his work on ridge regression analysis. The reader is referred to Figure 14 of section 4.1.2 for a plot of $r$ against $\mu$. It is of interest to note that for a given sphere, there is only one value of $\mu$ which will yield a maximum (minimum) value for the prediction variance. The proof of this result may be found in Draper (1963). Thus, if the design center is not identical to the center of the region of interest, there is only one combination of levels of the independent variables which produces the largest (smallest) variance of prediction on that sphere. 
Let $\underline{x}_{\max }$ denote the stationary point defined by $\underline{x}_{0}$ which yields the maximum prediction variance on the sphere and, similarly, $\underline{x}_{\operatorname{mm}}$ is the stationary point which yields the minimum prediction variance on the sphere. The maximum and minimum prediction variances on the sphere are respectively,

$$
\begin{aligned}
\max _{\underline{x} \in U_{r}} \frac{1}{\sigma^{2}} \operatorname{Var}(\hat{y}(\underline{x})) & =\underline{x}_{\max }^{*^{\prime}}\left(X^{*^{\prime}} X^{*}\right)^{-1} \underline{x}_{\max }^{*} \\
& =\frac{1}{N}+\sum_{i=1}^{k} \lambda_{i}\left(\frac{\lambda_{i} m_{i}}{\lambda_{i}-\mu_{\max }}\right)^{2}
\end{aligned}
$$

and

$$
\begin{aligned}
\min _{\underline{x} \in U_{r}} \frac{1}{\sigma^{2}} \operatorname{Var}(\hat{y}(\underline{x})) & =\underline{x}_{\min }^{*^{\prime}}\left(X^{*^{\prime}} X^{*}\right)^{-1} \underline{x}_{\min }^{*} \\
& =\frac{1}{N}+\sum_{i=1}^{k} \lambda_{i}\left(\frac{\lambda_{i} m_{i}}{\lambda_{i}-\mu_{\min }}\right)^{2}
\end{aligned}
$$

where $\mu_{\max }$ and $\mu_{\min }$ are the appropriate Lagrangian multipliers. The range of the prediction variances on the surface of a hypersphere of radius $r$ is given by

$$
\begin{aligned}
\operatorname{Rof} V(r) & =\frac{1}{\sigma^{2}} \operatorname{Var}\left(\hat{y}\left(\underline{x}_{\max }\right)\right)-\frac{1}{\sigma^{2}} \operatorname{Var}\left(\hat{y}\left(\underline{x}_{\min }\right)\right) \\
& =\sum_{i=1}^{k} \lambda_{i}\left\{\left(\lambda_{i} m_{i}\right)^{2}\left[\left(\frac{1}{\lambda_{i}-\mu_{\max }}\right)^{2}-\left(\frac{1}{\lambda_{i}-\mu_{\min }}\right)^{2}\right]\right\} \\
& =\sum_{i=1}^{k} \lambda_{i}^{3} m_{i}^{2}\left[\left(\frac{1}{\lambda_{i}-\mu_{\max }}\right)^{2}-\left(\frac{1}{\lambda_{i}-\mu_{\min }}\right)^{2}\right]
\end{aligned}
$$

\section{Case III : A second order model.}

When a second order model is used to approximate the true response function the variance of a predicted response is given by (3.2.5). The formulation of RofV requires the optimization of

$$
\frac{1}{\sigma^{2}} \operatorname{Var}(\hat{y}(\underline{x}))=\left[\underline{x}^{*^{\prime}}\left(X^{\prime} X\right)^{-1} \underline{x}^{*}\right]
$$


where $\underline{x}^{\circ}$ and the model-matrix $X$ are appropriate for a second order model, (see section 3.2.3). The optimization of this function over locations on the surface of a hypersphere is a very difficult task. It is necessary to use a computer search algorithm to find the extreme values of (3.2.11). There are many optimization packages available, but few can satisfactorily handle the problem of optimizing a nonlinear function subject to a nonlinear equality constraint on the variables.

For the illustrations presented in this thesis, the Modular In-core Nonlinear Optimization System (referred to as MINOS hereafter) computer package (Murtagh and Saunders (1983)) was used to generate maximum and minimum values of the prediction variance. The range of the prediction variances on the surface of a sphere is computed as the difference in the corresponding maximum and minimum values generated by MINOS.

The algorithm also provides a location at which the optimal value is obtained. There may be other points on the sphere which yield the same extreme value. These points may be found by selecting initial values for the independent variables in a different section of the sphere. Although there exist absolute maximum and minimum values for the prediction variance at locations on a sphere, the MINOS algorithm only provides local extremum. A thorough search of the surface of the sphere, however, should yield the absolute extremum for the problem. The MINOS algorithm is briefly described in Appendix A.3.

\subsubsection{The VofV on the Surface of a Hypersphere}

The variance of the prediction variances for locations on a sphere defined by (3.2.2) will be considered under the three cases described in section 3.2.3.

Case I : A first order model; the design center and region center are the same. 
Under the specifications established for case I in section 3.2.3, the average prediction variance on the surface of the k-dimensional hypersphere defined by $U_{r}$, the spherical variance, is

$$
\begin{aligned}
V^{r} & =\Psi \int_{U_{r}}\left(\frac{1}{N}+\sum_{i=1}^{k} \lambda_{i} z_{i}^{2}\right) d \underline{z} \\
& =\frac{1}{N}+\sigma_{2} \sum_{i=1}^{k} \lambda_{l} \\
& =\frac{1}{N}+\frac{r^{2}}{k} \sum_{i=1}^{k} \lambda_{i}
\end{aligned}
$$

where $\lambda_{t}, z_{i}, \mathrm{i}=1,2, \ldots, \mathrm{k}$ and the second spherical moment $\sigma_{2}=\Psi \int_{U_{r}} z_{i}^{2} d \underline{z}$ are defined in section 3.2.3. Observe that the average prediction variance on any sphere is a function of the average of the eigenvalues of the $\left(X^{\prime} X\right)^{-1}$ matrix. The squared deviation of the variance of an individual predicted response from the average variance of the predicted responses at locations on $U_{r}$ is, apart from $\sigma^{2}$,

$$
\begin{aligned}
{\left[\frac{1}{\sigma^{2}} \operatorname{Var}(\hat{y}(\underline{x}))-V^{r}\right]^{2} } & =\left[\sum_{i=1}^{k} \lambda_{i} z_{i}^{2}-\sigma_{2} \sum_{i=1}^{k} \lambda_{i}\right]^{2} \\
& =\sum_{i=1}^{k} \lambda_{i}^{2} z_{i}^{4}+2 \sum_{i=1}^{k} \sum_{j=1}^{k} \lambda_{i} \lambda_{j} z_{i}^{2} z_{j}^{2}-2 \sigma_{2}\left(\sum_{i=1}^{k} \lambda_{i} z_{i}^{2}\right)\left(\sum_{i=1}^{k} \lambda_{i}\right)+\sigma_{2}^{2}\left(\sum_{i=1}^{k} \lambda_{i}\right)^{2} .
\end{aligned}
$$

The variance of the variances of the predicted responses at locations on $U_{r}$ is the expected value of the expression above. Thus,

$$
\operatorname{Vof} V(r)=\sigma_{4} \sum_{i=1}^{k} \lambda_{i}^{2}+2 \sigma_{22} \sum_{i=1}^{k} \sum_{j=1}^{k} \lambda_{i} \lambda_{j}-\sigma_{2}^{2}\left(\sum_{i=1}^{k} \lambda_{i}\right)^{2}
$$

where $\sigma_{4}$ and $\sigma_{22}$ are spherical moments of order four. Substitution of

$$
\sigma_{2}=\frac{r^{2}}{k}, \sigma_{4}=\frac{3 r^{4}}{k(k+2)} \text { and } \sigma_{22}=\frac{r^{4}}{k(k+2)}
$$


above yields VofV on the surface of a hypersphere of radius $r$ for the first order model case with $\mathrm{k}$ factors present in the system as

$$
\begin{aligned}
V o f V(r) & =\frac{2 r^{4}}{k^{2}(k+2)}\left[(k-1) \sum_{i=1}^{k} \lambda_{i}^{2}-2 \sum_{i=1}^{k} \sum_{j=1}^{k} \lambda_{i} \lambda_{j}\right] \\
& =\frac{2 r^{4}}{k^{2}(k+2)} \sum_{i=1}^{k} \sum_{j=1}^{k}\left(\lambda_{i}-\lambda_{j}\right)^{2} \\
& =\frac{2 r^{4}}{k(k+2)} \sum_{i=1}^{k}\left(\lambda_{i}-\bar{\lambda}\right)^{2}
\end{aligned}
$$

where $\bar{\lambda}=\sum_{i=1}^{k} \lambda_{i} / k$.

It is interesting that VofV can be expressed simply in terms of the empirical variance of the eigenvalues of $\left(X^{\prime} X\right)^{-1}$. Again, the dispersion in the prediction variances on a hypersphere of radius $r$ depends on the dispersion in the eigenvalues of $\left(X^{\prime} X\right)^{-1}$. When the eigenvalues are the same, that is, when the design is rotatable, the VofV is equal to zero on any sphere. Thus, the VofV, as well as the RofV, provide reasonable measures of deviation from rotatability for a design.

A more appropriate measure of the variability in the variances of the predicted responses at locations on $U_{r}$ is the standard deviation of these variances. Let $\operatorname{SDofV}(r)=+\sqrt{\operatorname{Vof} V(r)}$ denote the standard deviation of the variances of the predicted responses at all locations which are a distance $r$ from the center of the region.

\section{Case II : A first order model; the design center and region center are not the same.}

Consider the evaluation of $V^{r}$ in the $w$-axis system. The prediction variances to be averaged in this case correspond to locations $\underline{w}=\left(\begin{array}{lllll}w_{1} & w_{2} & \ldots & w_{k}\end{array}\right)^{\prime}$ on the surface of the hypersphere of radius $r$ with center at $\underline{w}=\underline{0}-\underline{h}$. Let $U_{r}^{w}=\left\{\underline{w}: \sum_{i=1}^{k}\left(w_{i}+h_{i}\right)^{2}=r^{2}\right\}$ denote the surface of this hypersphere and $\Psi_{w}^{-1}=\int_{U_{r}^{w}} d \underline{w}$ be the surface area of $U_{r}^{w}$. Then, 


$$
\begin{aligned}
V^{r} & =\frac{\Psi_{w}}{\sigma^{2}} \int_{U_{r}^{w}} \operatorname{Var}(\hat{y}(\underline{w})) d \underline{w} \\
& =\Psi \int_{U_{r}}\left(\frac{1}{N}+\sum_{i=1}^{k} \lambda_{i} z_{i}^{2}-2 \sum_{i=1}^{k} \lambda_{i} m_{i} z_{i}+\sum_{i=1}^{k} \lambda_{i} m_{i}^{2}\right) d \underline{z} \\
& =\frac{1}{N}+\sigma_{2} \sum_{i=1}^{k} \lambda_{i}+\sum_{i=1}^{k} \lambda_{i} m_{i}^{2} \\
& =\frac{1}{N}+\frac{r^{2}}{k} \sum_{i=1}^{k} \lambda_{i}+\sum_{i=1}^{k} \lambda_{i} m_{i}^{2}
\end{aligned}
$$

by a transformation of variables and (3.2.4). The interim steps required in this formulation appear in Appendix A.4.

Now,

$$
\begin{aligned}
\operatorname{Vof} V(r)= & \Psi_{w} \int_{U_{r}^{w}}\left[\operatorname{Var}(\hat{y}(\underline{w})) / \sigma^{2}-V^{r}\right]^{2} d \underline{w} \\
= & \Psi \int_{U_{r}}\left[\left(\frac{1}{N}+\sum_{i=1}^{k} \lambda_{i} z_{i}^{2}-2 \sum_{i=1}^{k} \lambda_{i} m_{i} z_{i}+\sum_{i=1}^{k} \lambda_{i} m_{i}^{2}\right)\right. \\
& \left.-\left(\frac{1}{N}+\sigma_{2} \sum_{i=1}^{k} \lambda_{i}+\sum_{i=1}^{k} \lambda_{i} m_{i}^{2}\right)\right]^{2} d \underline{z}
\end{aligned}
$$

The second equality results from a transformation of variables and the results of equations (3.2.4) and (3.2.14). After simplification, the variance of the variances of the estimated responses on the surface of a hypersphere of radius $r$ under case II is

$$
\operatorname{Vof} V(r)=\frac{2 r^{4}}{k(k+2)} \sum_{i=1}^{k}\left(\lambda_{l}-\bar{\lambda}\right)^{2}+4 \frac{r^{2}}{k} \sum_{i=1}^{k} \lambda_{i}^{2} m_{i}^{2}
$$

where $\bar{\lambda}=\sum_{i=1}^{k} \lambda_{i} / k$. The reader is referred to Appendix A.5 for details.

If the design center corresponds to the center of the hypersphere, then, not surprisingly, the $V^{r}$ and VofV given here correspond to the $V^{r}$ and VofV derived under case $I$. It is of more interest to note that the $V^{r}$ and VofV for a design which is not centered about the origin of the region of 
interest are inflated over those for the same design which has been centered to the origin. Since the eigenvalues are the same for both designs, the amount by which the $V^{r}$ and VofV differ for the two designs increases as the design is removed further from the region center. Thus, it is to the researcher's advantage to select a design which is centered about the center of the region of interest to achieve more precise estimates of the response as well as more consistent estimation of the response on spheres.

\section{Case III : A second order model.}

In general, the spherical variance can be written as

$$
V^{r}=\operatorname{tr}\left\{S\left(X^{\prime} X\right)^{-1}\right\}
$$

where $\operatorname{tr}\{\cdot\}$ denotes the trace of a matrix, and $S$ is the spherical region moment matrix defined by (3.2.6) corresponding to the model. The matrix $X$ in this definition is the full model-matrix as described in section 2.2.3. This result is given by Hussey, Myers and Houck (1987) in their paper introducing the spherical variance. The form of the matrix $S$ for a second order model in $\mathrm{k}$ variables is given there. In this form, and with knowledge of the matrix $S$, the $V^{r}$ is easily computed in the second order model case. However, because of the difficulty in deriving a form for the VorV when the model is second order, only the $V^{r}$ and VofV for a second order model in $\mathrm{k}=2$ variables will be considered here. It was decided not to pursue the development of the VofV further since this criterion does not lend itself as well to graphical representation as does the $V^{r}$ and, as will subsequently be shown, the RofV.

So, suppose that a second order model in two variables is fitted to the response of the system. In this situation, $\underline{x}^{*}=\left(\begin{array}{llllll}1 & x_{1} & x_{2} & x_{1}^{2} & x_{2}^{2} & x_{1} x_{2}\end{array}\right)^{\prime}$ and $S$ has the form 


$$
S=\left[\begin{array}{cccccc}
1 & 0 & 0 & \sigma_{2} & \sigma_{2} & 0 \\
0 & \sigma_{2} & 0 & 0 & 0 & 0 \\
0 & 0 & \sigma_{2} & 0 & 0 & 0 \\
\sigma_{2} & 0 & 0 & \sigma_{4} & \sigma_{22} & 0 \\
\sigma_{2} & 0 & 0 & \sigma_{22} & \sigma_{4} & 0 \\
0 & 0 & 0 & 0 & 0 & \sigma_{22}
\end{array}\right] .
$$

Let the elements of $\left(X^{\prime} X\right)^{-1}$ be denoted by $c^{i j}$ for $i, j=0,1,2,3,4,5$. Here the superscript $i$ indicates the row and $\mathrm{j}$ the column in which the element $c^{\mathrm{j}}$ appears. It turns out (see Appendix A.6) that the spherical variance in terms of the spherical moments and elements of $\left(X^{\prime} X\right)^{-1}$ is

$$
V^{r}=c^{00}+\sigma_{2}\left[\sum_{i=1}^{2}\left(2 c^{0, i+2}+c^{i i}\right)\right]+\sigma_{4} \sum_{i=1}^{2} c^{i+2, i+2}+\sigma_{22}\left(c^{55}+2 c^{34}\right)
$$

Consider now the general form of the VofV as defined in (3.2.2). It is not difficult to show that

$$
\begin{aligned}
\operatorname{Vof} V(r) & =\Psi \int_{U_{r}}\left[\frac{1}{\sigma^{2}} \operatorname{Var}(\hat{y}(\underline{x}))\right]^{2} d \underline{x}-\left(V^{\prime}\right)^{2} \\
& =\Psi \int_{U_{r}} \underline{x}^{\prime}\left(X^{\prime} X\right)^{-1} \underline{x x^{\prime}}\left(X^{\prime} X\right)^{-1} \underline{x} d \underline{x}-\left(V^{\prime}\right)^{2} \\
& =\operatorname{tr}\left\{T\left(X^{\prime} X\right)^{-1}\right\}-\operatorname{tr}\left\{S\left(X^{\prime} X\right)^{-1}\right\}
\end{aligned}
$$

where $T=\Psi \int_{U_{Y}} \underline{x x^{\prime}}\left(X^{\prime} X\right)^{-1} \underline{x x^{\prime}} d \underline{x}$. Note that the * superscript on the vector $\underline{x}$ has been dropped to ease the burden of notation.

For the case of a second order model, $T$ contains spherical region moments through order eight as well as the elements of the $\left(X^{\prime} X\right)^{-1}$ matrix. In this case, the VofV is an eighth degree polynomial in the radius $r$ of the hypersphere. It has not been reproduced here since it is lengthy and does not provide any insight into the characteristics of this dispersion measure. A computer 
program which will evaluate (3.2.17) under the conditions of case III for two variables over a range of radii is presented in Appendix A.7.

\subsection{Illustrations of the Use of the Spherical Variance and}

\section{PVD Measures}

The spherical variance and PVD measures were developed to aid the researcher in choosing a satisfactory design for the experimental situation. These criteria facilitate the comparison of competing designs as well as allow the investigation of the prediction properties of a design. The examples presented in this section were chosen to illustrate how these measures can be used to answer questions typically posed by the researcher. In particular, the effects of losing one or more design runs, inaccurate measurement of the levels of the independent variables and augmenting a design point to an already existing design on the prediction capability of several first order designs will be examined. A comparative study of some families of second order designs is presented in Chapter V.

\subsubsection{A Plot Depicting Prediction Capability on Spheres}

The spherical variance, VofV and RofV criteria, each of which describes some property of the prediction variances on the surface of a sphere, can be represented on a graph for varying values of the radius of the sphere. It would be most practical to depict the average prediction variances and the dispersion in the variances on spheres in a single plot. It has already been shown that the average behavior of prediction variances on spheres is readily viewed in a plot of the $V^{r}$ versus the radius of the sphere, $r$. The simplest way to include the dispersion in the prediction variances in 
this picture is to plot the maximum and minimum prediction variances corresponding to each radius. The distance between the curves of maximum and minimum prediction variances at a given radius $r$ is the range of the prediction variances on the sphere of radius $r$. Together, the three curves, $V^{r}$, maximum and minimum variances, provide a clear and concise description of the prediction capability on spheres for a design.

Alternatively, 'confidence bands' of the form $V^{r} \pm c S D o f V(r)$ for some constant $c$ about the spherical variance curve could be included to indicate the variability in the prediction variances for each $r$. Such bands will not be considered further since it is apparent that they are considerably more difficult to interpret than the graph of maximum and minimum prediction variances on spheres.

\subsubsection{Illustration of the Effects of Loss of Design Points}

Suppose that during the exploration of the response of a system in three variables a first order model is fitted to the response in some region of the independent variables. At this stage it is reasonable to employ a $2^{3}$ factorial design to analyze the behavior of the response in the region. The $2^{3}$ factorial design, being an orthogonal design, yields independent estimates of the coefficients of the first order model and equal variances of the predicted responses on spheres about the region center.

If the researcher suspects that some of the design points might be lost during experimentation, he or she may wish to make additional experimental runs to soften any adverse effects the loss of design points might have on the prediction capability of the design. Usually, extra experimental runs of this sort are taken at the center of the region. In this way, the orthogonality and rotatabilty properties are maintained for the $2^{3}$ factorial design, and for any orthogonal and rotatable first order design. It has already been observed that the increased precision on spheres gained by adding two 
experimental runs in the center of the $2^{3}$ factorial design is not enough to merit the additional cost of the two center points, (see Figure 3, section 3.1.4). However, it would be worthwhile to determine if the center runs will provide protection against the deterioration of the prediction capability of the $2^{3}$ design if one or more design points are lost.

Consider the investigation of the prediction variance properties on spheres for a $2^{3}$ factorial design in which two design points have been lost. For this illustration, suppose the resulting design is the second design, D2, given in the example of section 3.1.5. This design has six design points and no center points. The curves of maximum and minimum prediction variances for this design are depicted in Figure 5. The spherical variance is represented as the solid curve between the maximum and minimum prediction variance curves. The curves were constructed under the formulation of case I since this particular design is centered about the region of interest.

The comparison of this design with the full $2^{3}$ factorial design presented in Figure 3 clearly indicates that the estimation of the response is typically much worse when information has been lost. Note that the minimum prediction variance curve corresponding to the $2^{3}$ design with two missing points lies above the prediction variance curve for the full factorial. Thus, the accuracy of prediction for the $2^{3}$ factorial design is everywhere better than that of the $2^{3}$ factorial with two missing. Figure 5 shows that the accuracy of the predicted responses at locations on spheres is somewhat inconsistent when predicting near the center of the region but worsens considerably as the response is estimated on spheres far from the region center. Prediction of the response is worst in the directions of the missing design points $\left(\begin{array}{lll}1 & 1 & 1\end{array}\right)$ and $\left(\begin{array}{lll}-1 & -1 & -1\end{array}\right)$. One set of the locations of maximum prediction variance on spheres is pictured in Figure 7. For a given radius the maximum prediction variance is attained where the three variables take on the same values on the sphere. Hence, the lines depicting the values of $x_{1}, x_{2}$ and $x_{3}$ in Figure 7 coincide in this case.

The variances plotted in Figure 5 have been weighted by the number of design points, $N=6$ here, to permit the comparison of this design with a $2^{3}$ factorial design with the same two points lost and two additional runs in the center. The weighted spherical variance and maximum 
and minimum prediction variances over locations on the surface of a hypersphere of radius $r$ are given in Figure 6 for this design; $N=8$ here. Careful scrutiny of Figures 5 and 6 reveals that the larger design is not as efficient as the smaller design in predicting the response on spheres. Thus, the addition of two center runs to a $2^{3}$ factorial would not provide suitable protection to the prediction properties on spheres against the loss of two design points. Consequently, in this situation, and especially if experimental runs are costly, the researcher would do well to save the additional runs intended for the center to be used to improve the prediction capability of the design in a subsequent stage of experimentation.

It is not surprising to observe a loss in precision when information is lost, particularly when the sample size is small at the start. The spherical variance and PVD measures enable the researcher to gauge the extent of the damage to the prediction capability of the original design. With these criteria the researcher can also investigate the consequences of implementing a different design and, thereby, choose the most favorable experimental strategy.

\subsubsection{Illustration of the Effects of Improper Design Settings}

Sometimes in an experiment the levels of the variables are improper. That is, there is error in the measurement of the variables caused by a faulty measurement device or inaccurate reading of the measurement. For instance, if the thermostat which regulates the temperature of a chemical process routinely gives readings below the actual temperature, the actual design settings used in an experiment involving the temperature of the process are not those prescribed in the experimental design plan. The properties of the design used in the experiment are usually not known since, even if it is known that the settings are improper, the true values used are not known to the researcher. However, the effects of improper settings on the prediction capability of a design can be examined in general with a few case examples. 


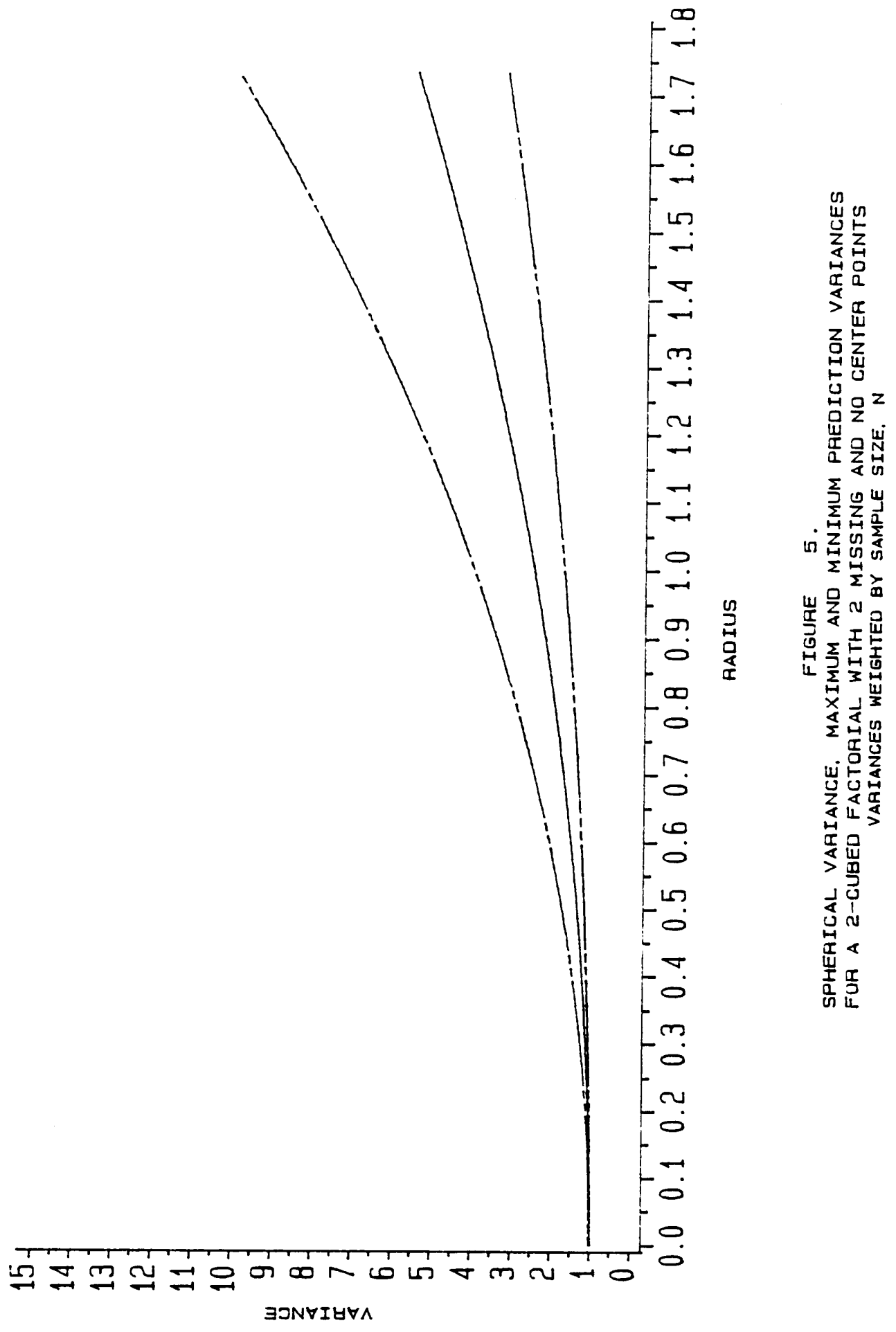




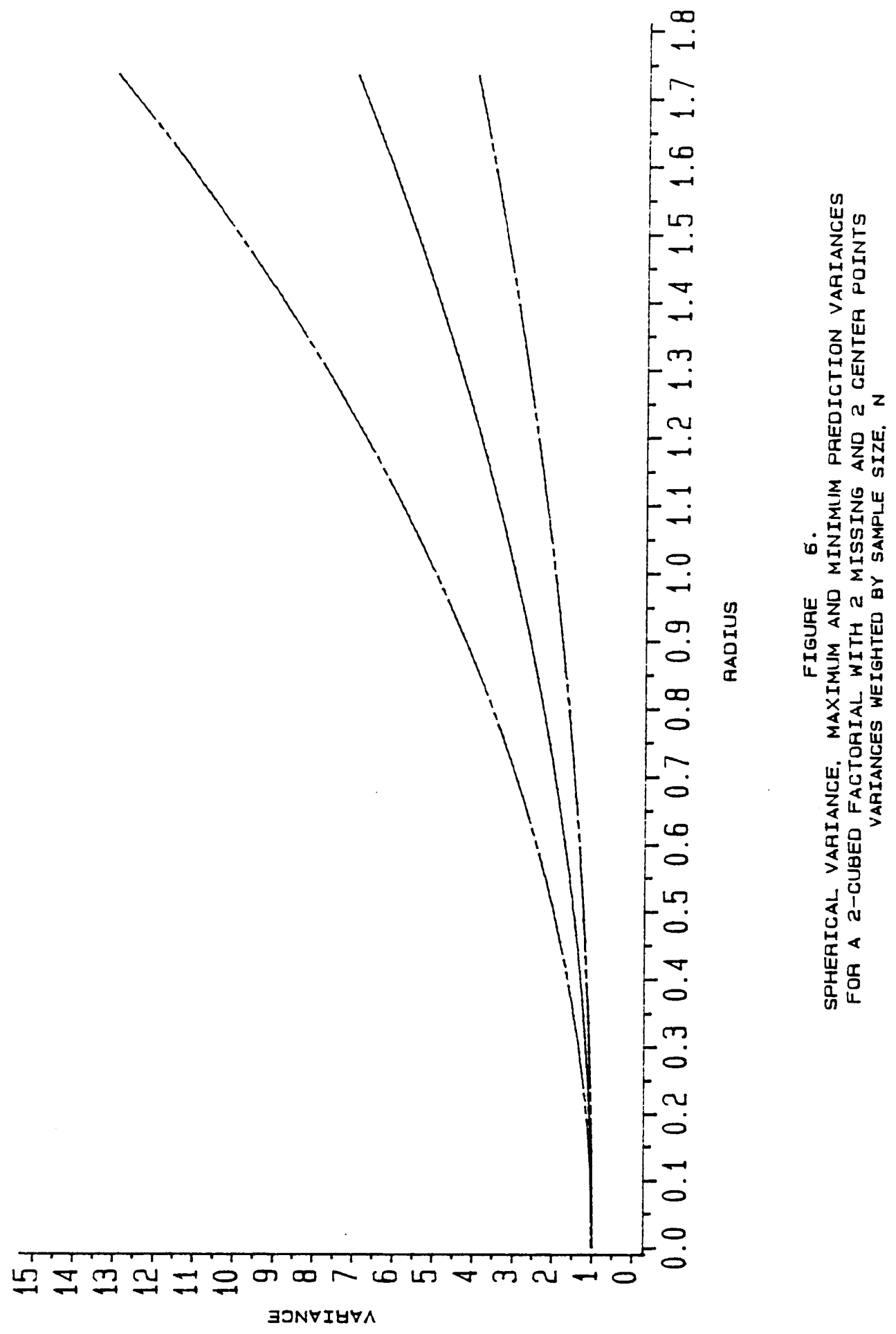




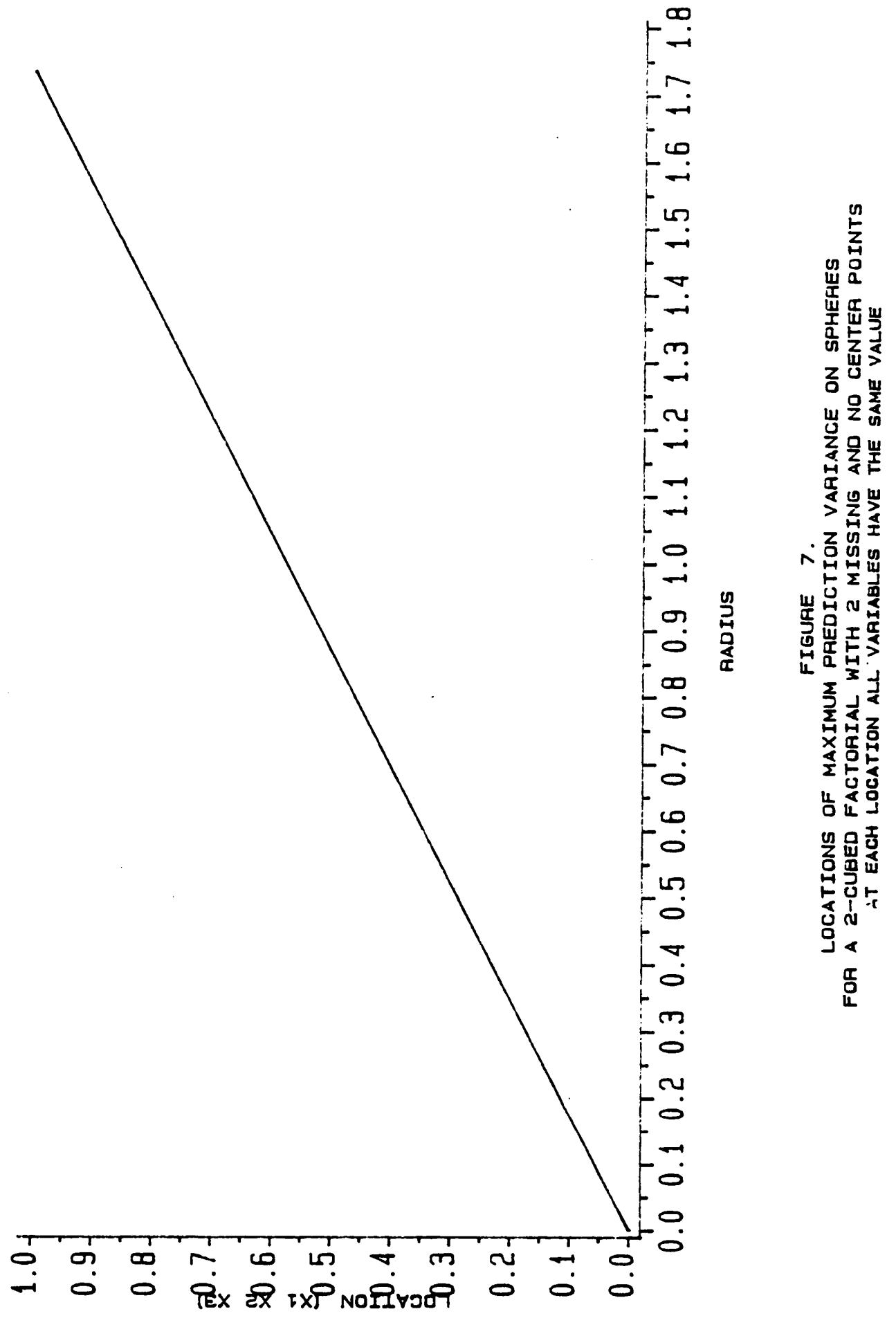


As an example, consider a $2^{4}$ factorial design with one center point used to fit a first order model in some region of the design variables. Suppose the levels of some of the variables are subject to inaccurate measurement. For purposes of illustration, two types of improper settings will be considered. In the first type the levels of all the variables are measured improperly and the actual values obtained deviate from the values specified in the design plan by one to ten percent. Thus, if the required level of a variable is $x_{1}=1$, say, the value of $x_{1}$, measured as $x_{1}=1$, is actually something between 0.99 and 0.90 . The design given by (3.3.1) exhibits this type of improper settings.

Improper settings occur in only two of the four variables in the second classification of improper settings. The measurement of the levels in these two variables, however, is highly inaccurate; some values are between twenty-five and fifty percent of their measured values. That is, a variable recorded at a level of 1 may actually be between 0.75 and 0.50 . A design which can be classified as having this type of improper settings appears in (3.3.2). 


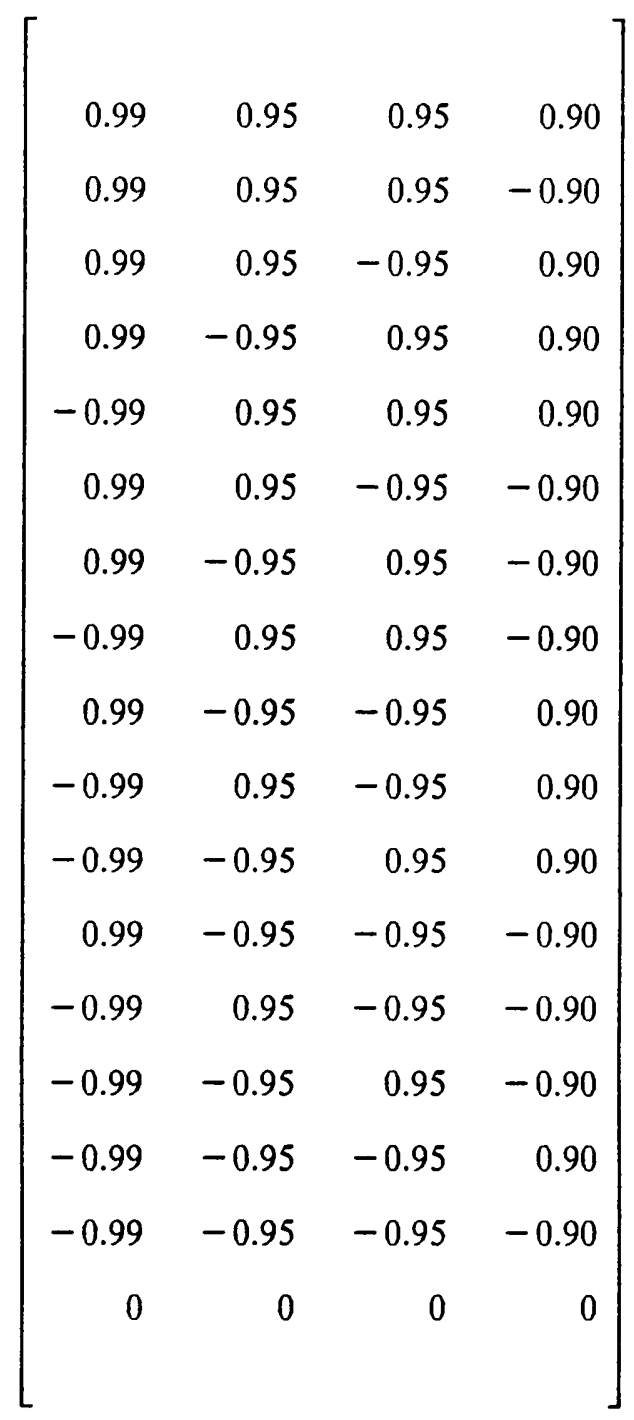




$$
\left[\begin{array}{rrrr}
1 & 1 & 1 & 1 \\
1 & 1 & 1 & -1 \\
1 & 1 & -0.6 & 1 \\
1 & -0.5 & 1 & 1 \\
-1 & 1 & 1 & 1 \\
1 & 1 & -0.6 & -1 \\
1 & -1 & 1 & -1 \\
-1 & 0.5 & 1 & -1 \\
1 & -1 & -0.6 & 1 \\
-1 & 0.7 & -0.6 & 1 \\
-1 & -1 & 1 & 1 \\
1 & -1 & -0.6 & -1 \\
-1 & 1 & -0.6 & -1 \\
-1 & -1 & 1 & -1 \\
-1 & -1 & -0.6 & 1 \\
-1 & -0.7 & -0.6 & -1 \\
0 & 0 & 0 & 0 \\
& & &
\end{array}\right]
$$

These $2^{4}$ factorial designs with improper settings will be used to investigate the effects of the two types of improper settings on the prediction capability on spheres of a $2^{4}$ factorial design.

The spherical variances for the three designs are compared in Figure 8. It is apparent that on the average, at least, the loss of precision due to improper design settings is greatest when the levels are off by as much as twenty-five to fifty percent from their recorded values. A comparison of the more complete pictures of prediction capability on spheres for the two designs with improper settings in Figures 9 and 10 reveals too that there is a much wider range of variances of the predicted responses on spheres for this design. The accuracy of prediction on spheres is highly inconsistent 


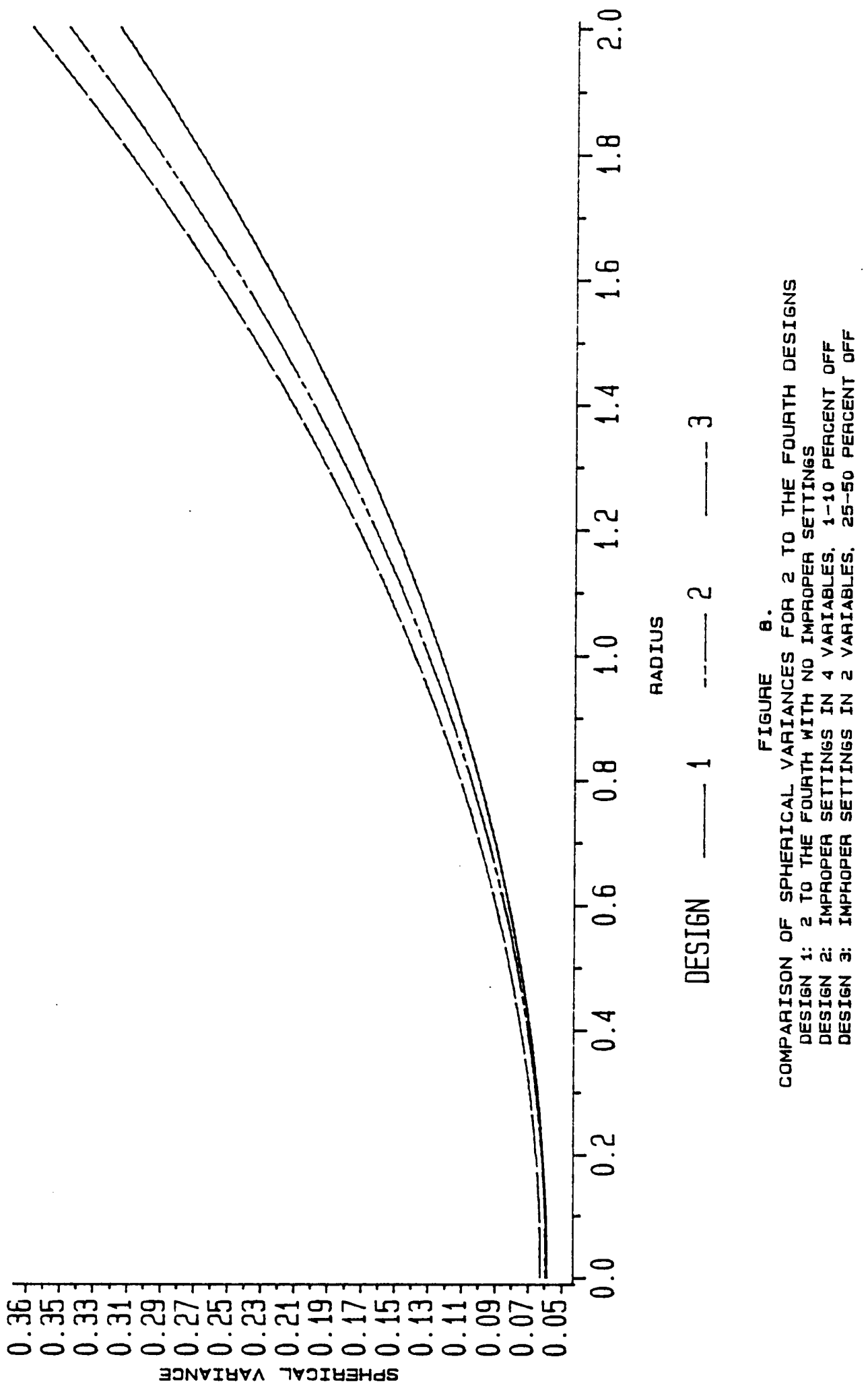




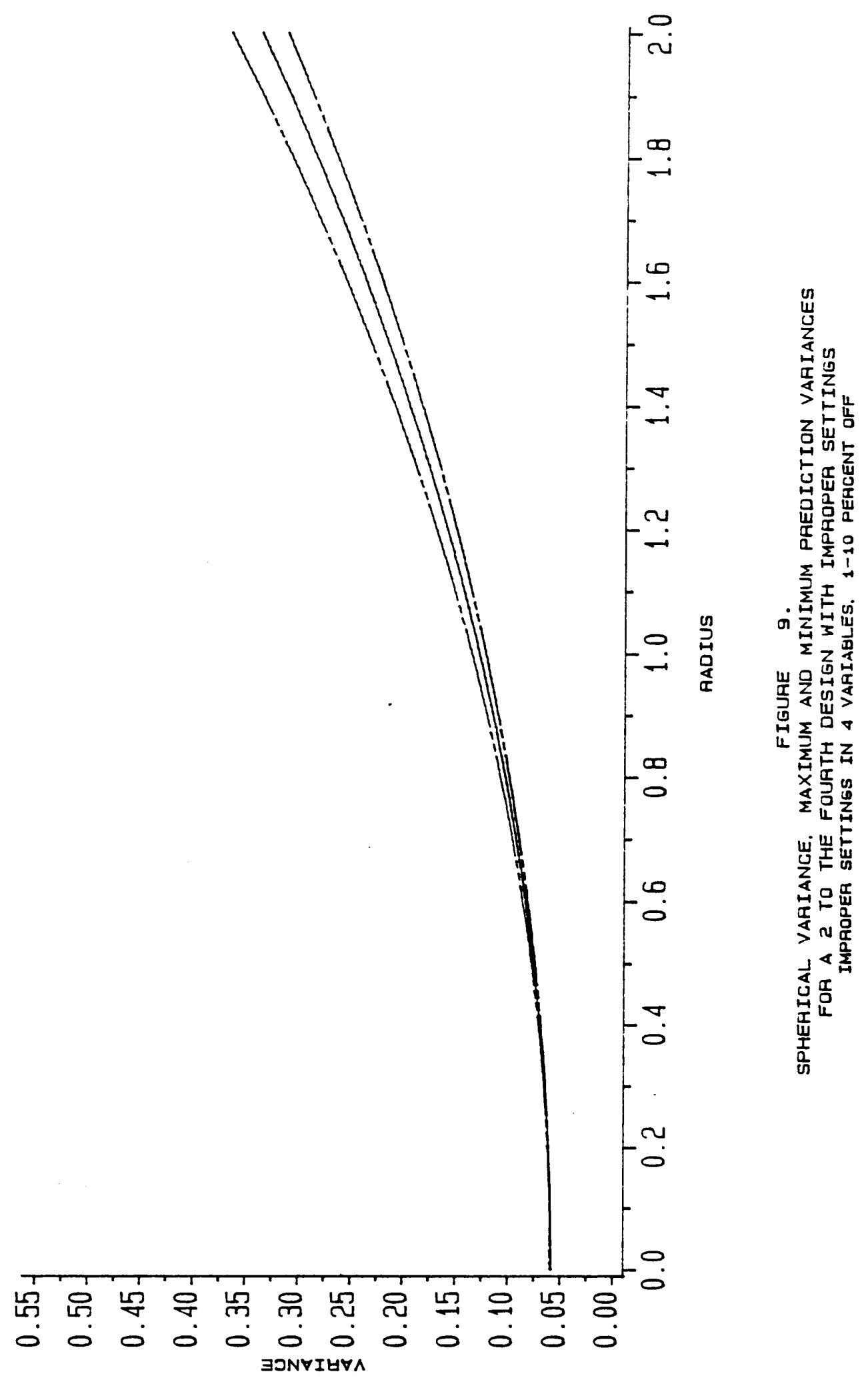




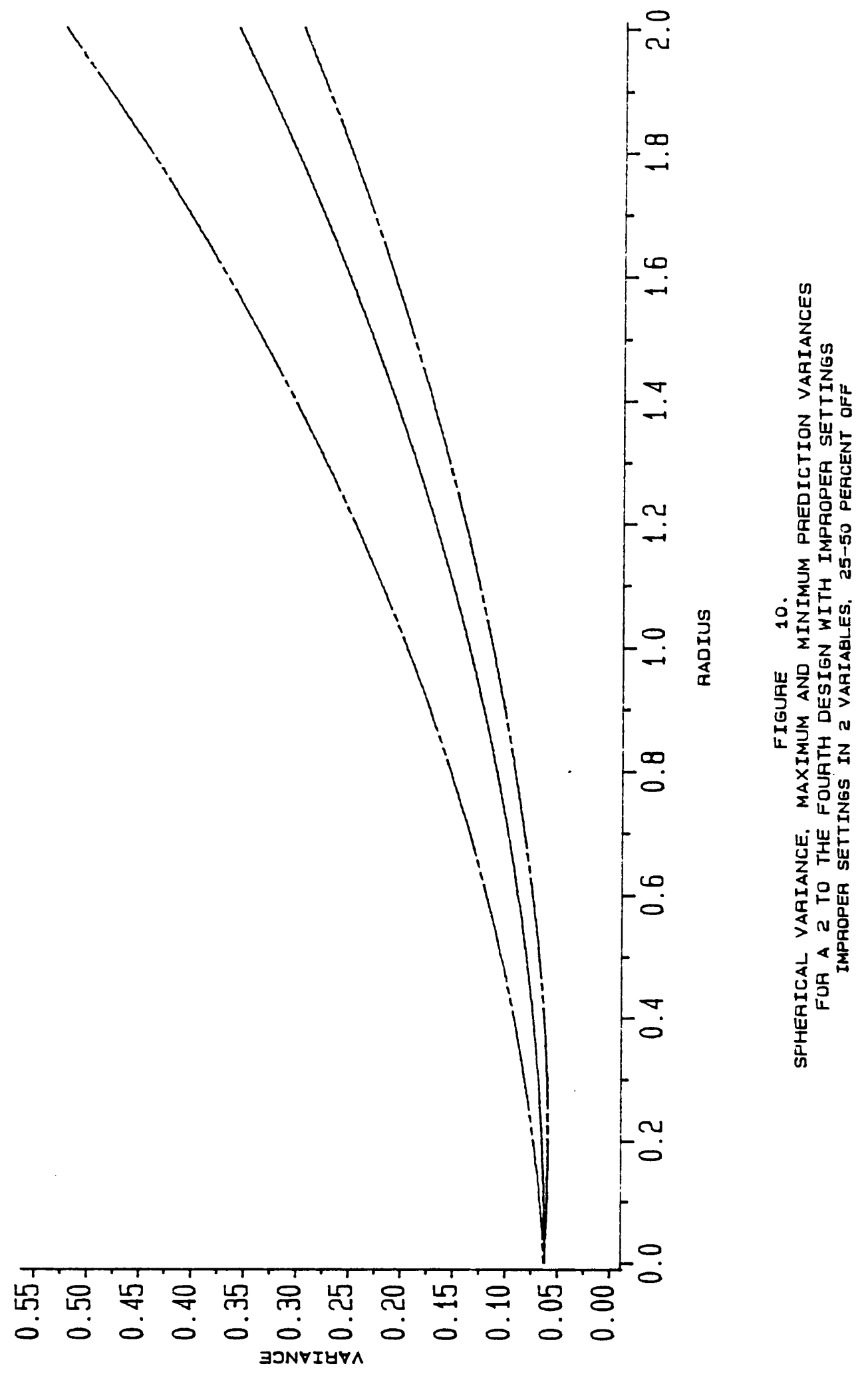


for all spheres. For the design representing the first type of improper settings there is little dispersion in the prediction variances at locations on a sphere.

In both cases, the spherical variance and maximum and minimum prediction variance curves allowed an easy assessment of the effects of improper settings on the prediction capability of the full factorial design. Using more extensive case studies, general statements about the effects of different types of improper settings on the prediction variances of a particular design could be made.

\subsubsection{Illustration of the Effects of Augmenting a Point to the Design}

The exploration of a response surface is a sequential process. Often one or more experimental runs are taken to supplement a design used in the previous stage of experimentation. Points may be augmented to an existing design for many reasons. Additional design points may be required to estimate the parameters of a higher order model or to replace lost points, or additional points may be taken in an effort to improve the statistical properties of the design.

This example will illustrate the effects of augmenting one design point to a design in order to maximize $\left|X^{\prime} X\right|$ at that stage. The resulting design will be D-optimal among all designs which consist of the original design plus one additional point from the region of the independent variables. Dykstra (1971) showed that such a conditionally D-optimal design can be achieved by placing the additional point at the location in the region which yields the largest prediction variance under the original design. The spherical variance and maximum and minimum prediction variance curves will be used to examine the consequences of this course of action on the prediction variance properties on spheres of the design.

Suppose now that the design given in (3.3.3) is used to fit a first order model in four variables to the response of a system. The plot depicting the nature of the prediction in Figure 11 clearly indicates that the response is not being estimated very well with this design. An additional exper- 
imental run will be taken at the location of maximum prediction variance in an effort to improve this picture.

$$
\left[\begin{array}{rrrr}
1 & 1 & 1 & 1 \\
1 & 1 & 1 & -1 \\
1 & 1 & 1 & 1 \\
-1 & -1 & -1 & 1 \\
1 & 1 & 1 & 1 \\
1 & 1 & -1 & -1 \\
1 & -1 & 1 & -1 \\
1 & 1 & 1 & -1 \\
-1 & -1 & -1 & 1 \\
-1 & -1 & -1 & 1 \\
-1 & -1 & 1 & 1 \\
1 & 1 & -1 & -1 \\
-1 & -1 & 1 & -1 \\
-1 & 1 & -1 & -1 \\
-1 & -1 & -1 & 1 \\
-1 & -1 & -1 & -1 \\
0 & 0 & 0 & 0 \\
& & &
\end{array}\right]
$$

Locations of the maximum prediction variances on spheres in a hypersphere of radius 2 appear in Figure 12. The maximum prediction variance occurs on the perimeter of this region at levels $x_{1}=1.49809, x_{2}=-1.19679, x_{3}=-0.560883$ and $x_{4}=0.0939163$. This point is augmented to the design in (3.3.3). The spherical variances and maximum and minimum prediction variances on spheres for the augmented design are plotted in Figure 13. The additional point did much to improve the maximum prediction variances and, therefore, the spherical variances of the 


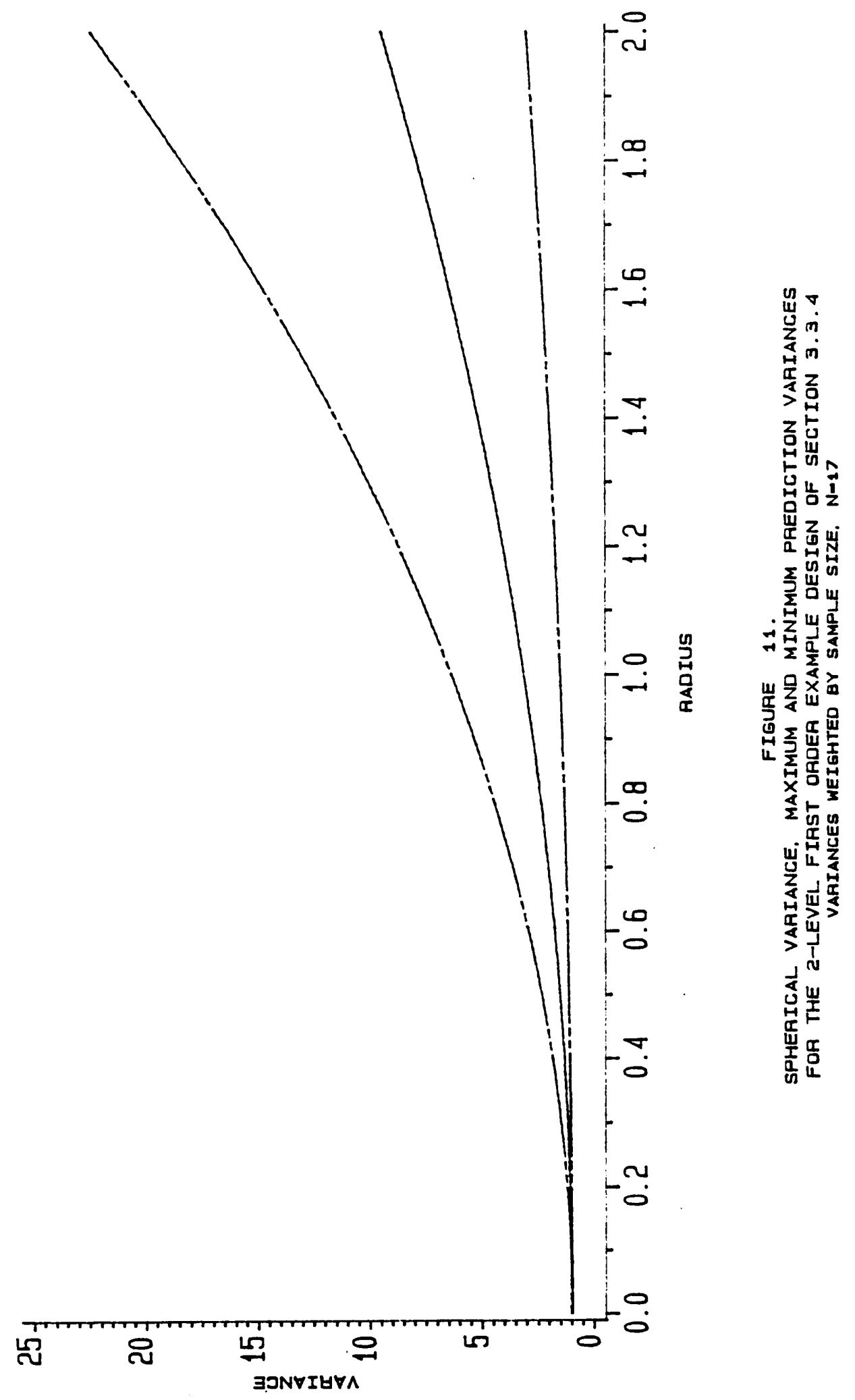




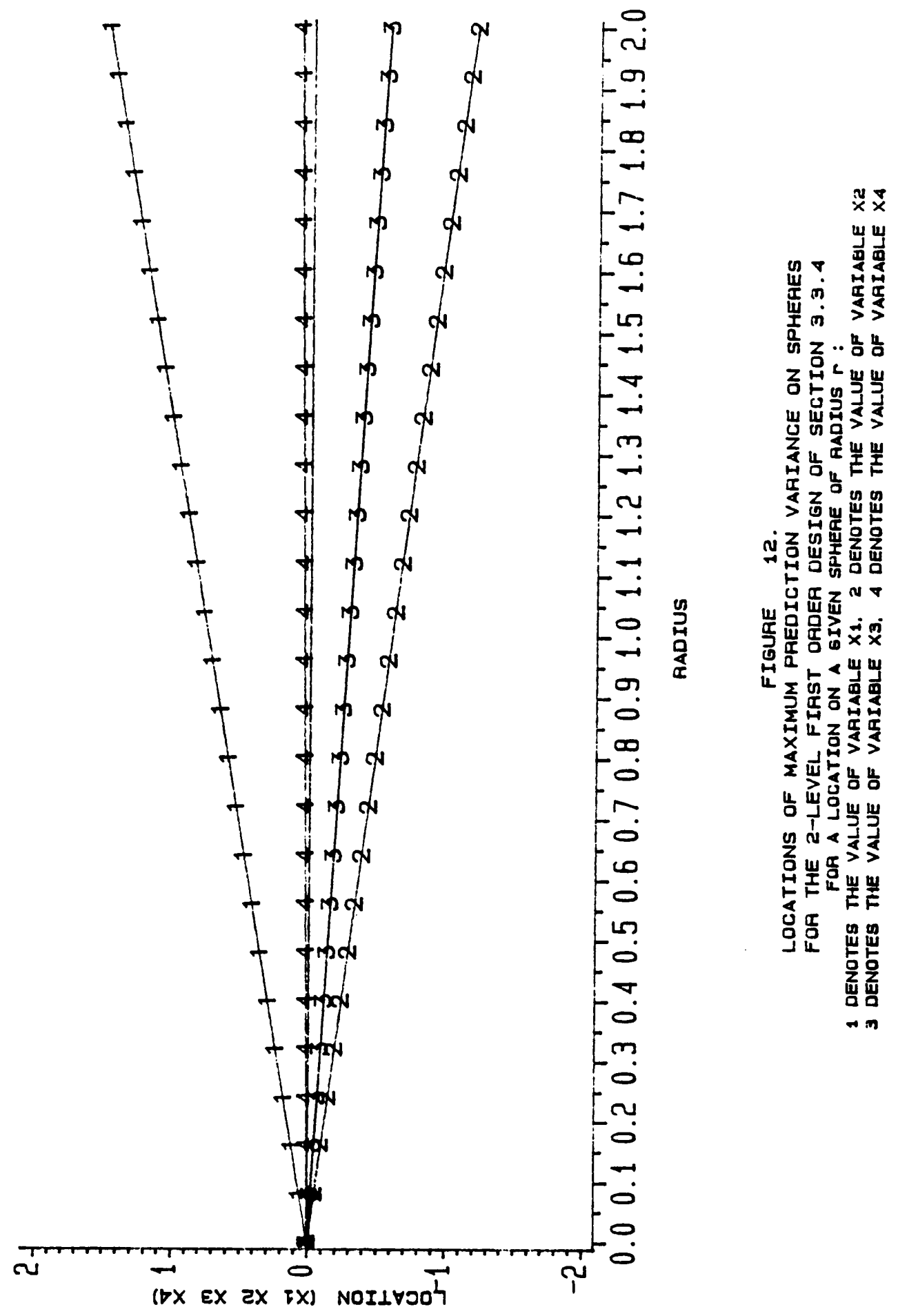




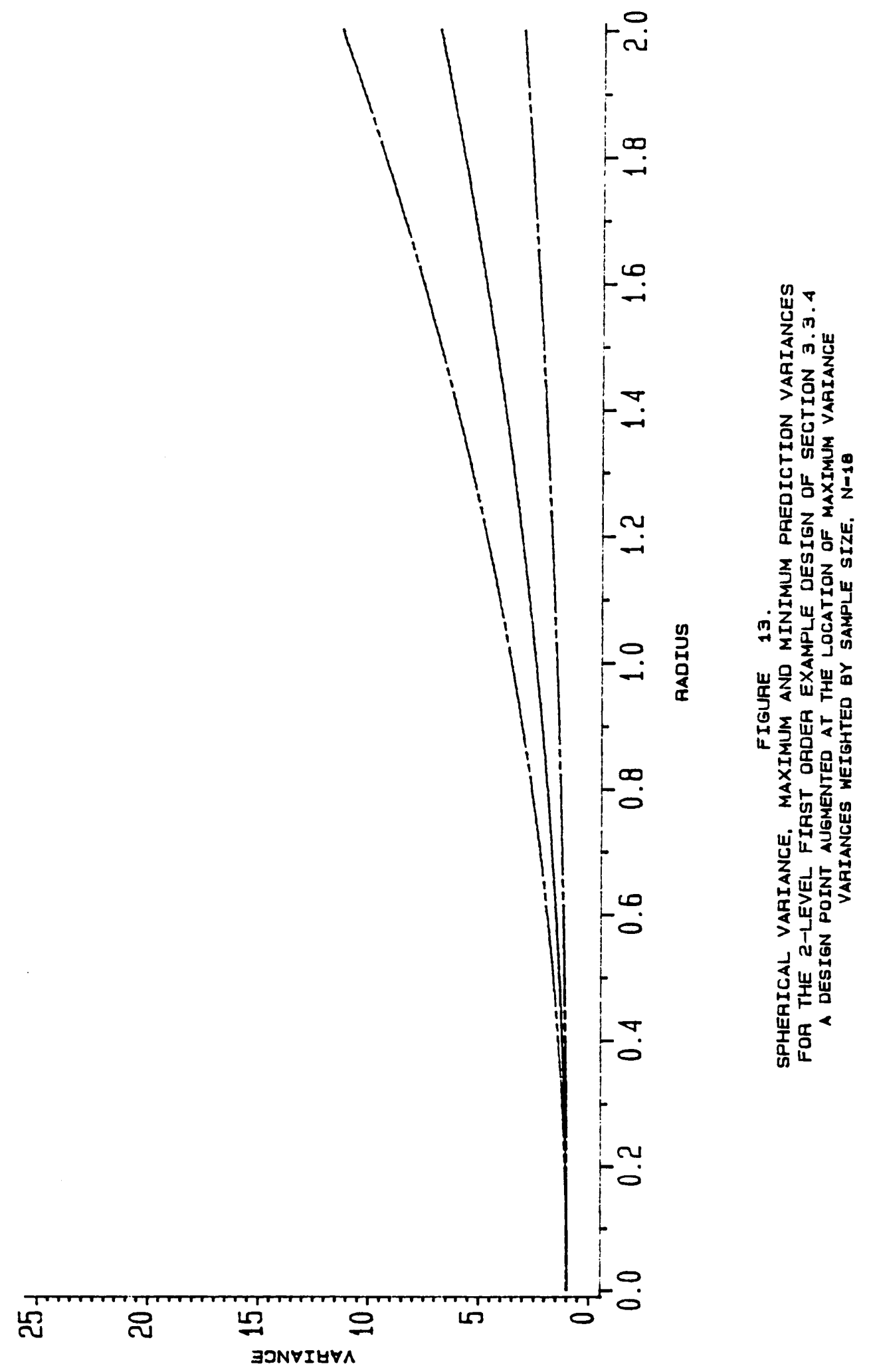


design. The maximum prediction variance in the region is reduced by more than half with the additional point at the location of maximum prediction variance. The location of the maximum prediction variance in the region has not changed however. Note that the minimum prediction variance curve was not effected by the addition of the point.

The addition of a point to maximize $\left|X^{\prime} X\right|$, since it reduces the maximum prediction variance, also improves the prediction capability of the design. However, this may not be the most effective strategy to employ to achieve better prediction capability on spheres. A different criterion for selecting the location of a point to be augmented to an existing design will be discussed in Chapter IV. Also in Chapter IV the characteristics of the spherical variance and prediction variance dispersion measures as functions of the radius will be investigated. Some statistical invariance properties of the measures will be set forth there as well. 


\title{
Chapter IV
}

\section{Properties of the Spherical Variance and PVD}

\author{
Measures
}

\subsection{Trends in the Prediction Variance Functions}

The spherical variance and PVD measures are functions of the distance, $r$, from the center of the region of the independent variables at which the responses are being estimated. In this section, the behavior of these prediction variance functions will be examined as the responses are estimated further from the region center, that is, on hyperspheres of increasing radius. Trends in the spherical variance and PVD measures will be discussed when the assumed model for the response is first order, (case I and case II). Results pertaining to the behavior of these functions as $r$ increases have not been obtained when the fitted model is second order. In this case, trends in the $V^{r}$ and maximum and minimum prediction variance curves may be observed for the second order designs described in section 2.4. These illustrations appear in Chapter V. 


\subsubsection{Trends in the Spherical Variance as $r$ Increases}

When a first order model in $\mathrm{k}$ variables is used to approximate the response function the spherical variance corresponding to a particular design is given by

$$
V^{r}=\frac{1}{N}+\frac{r^{2}}{k} \sum_{i=1}^{k} \lambda_{i}+\sum_{i=1}^{k} \lambda_{i} m_{i}^{2}
$$

This representation of the $V^{r}$ is appropriate to both case I and case II as described in section 3.2.3. If the design center is the same as the center of the region of the independent variables (case I), $m_{i}=0$ for all $\mathrm{i}=1,2, \ldots, \mathrm{k}$ and the general form of the $V^{r}$ given above reduces to the specific form for case I given by equation (3.2.12).

In either case, $\lambda_{1}, \lambda_{2}, \ldots, \lambda_{k}$, which depend on the design, are the eigenvalues of a positive definite matrix. Hence, $\lambda_{i}>0$ for all $\mathrm{i}=1,2, \ldots, \mathrm{k}$ and $\sum_{i=1}^{k} \lambda_{i}>0$ for any response surface design. The rate of change of the spherical variance as the radius, $r$, increases is

$$
\frac{\partial V^{r}}{\partial r}=2 r \sum_{i=1}^{k} \lambda_{i} / k=2 r \bar{\lambda}
$$

which is positive for $r>0$ and is equal to zero if and only if $r=0$. Thus, as the radius of the hypersphere over which the prediction variances are being averaged increases, the spherical variance also increases. On the average the precision of the estimated responses diminishes as one predicts further from the center of the region in the first order model case. The design with the smallest possible sum of the eigenvalues among all competing designs will yield the least amount of increase in the average prediction variance on a sphere per unit increase in $r$.

Under the conditions of case I, the design which has minimum $\sum_{i=1}^{k} \lambda_{i}$ among all designs of size $N$ also has minimum $V^{r}$ per radius $r$ among those designs. Note that, when the design center is the same as the region center, $\sigma^{2}\left(1 / N+\sum_{i=1}^{k} \lambda_{t}\right)$ is the sum of the variances of the estimated coefficients of the first order model. In this case, 


$$
\begin{aligned}
\sum_{i=0}^{k} \operatorname{Var}\left(\hat{\beta}_{i}\right) / \sigma^{2} & =\operatorname{tr}\left\{\left(X^{*} X^{*}\right)^{-1}\right\} \\
& =\frac{1}{N}+\sum_{i=1}^{k} \lambda_{i}
\end{aligned}
$$

where all terms are defined in section 3.2 .3 under case I.

To minimize the sum of the variances of the coefficients apart from $\sigma^{2}$ for fixed $N$, the design should be chosen which has the smallest value of $\sum_{i=1}^{k} \lambda_{i}$. This design is then the A-optimal design among all first order designs of size $N$. Thus, for case I, the design which has minimum spherical variance per radius among all first order designs with $N$ design points is the A-optimal design.

\subsubsection{Trends in the RofV as $r$ Increases}

Case I : A first order model; the design center and region center are the same.

Consider first the behavior of the maximum and minimum prediction variances on a sphere as the radius of the sphere increases. Recall that,

$$
\max _{\underline{x} \in U_{r}} \frac{1}{\sigma^{2}} \operatorname{Var}(\hat{y}(\underline{x}))=\frac{1}{N}+\lambda_{k} r^{2}
$$

and

$$
\min _{\underline{x} \in U_{r}} \frac{1}{\sigma^{2}} \operatorname{Var}(\hat{y}(\underline{x}))=\frac{1}{N}+\lambda_{1} r^{2}
$$

where $U_{r}=\left\{\underline{x}: \sum_{i=1}^{k} x_{i}^{2}=r^{2}\right\}$ defines the surface of the hypersphere, and $\lambda_{k}$ and $\lambda_{1}$ are respectively the largest and smallest eigenvalues of $\left(X^{\prime} X\right)^{-1}$. Only the case where $\lambda_{k}>\lambda_{1}$ will be considered here. If $\lambda_{k}=\lambda_{1}$ the design is rotatable, the maximum and minimum prediction variance curves coincide and the RofV is equal to zero for all $r$. 
It is apparent that both the maximum and minimum prediction variance curves are increasing functions of the radius, $r$. However, since $\lambda_{k}>\lambda_{1}$, the maximum prediction variances are increasing more quickly than the minimum prediction variances. Thus, the difference in the maximum and minimum prediction variances on a sphere, that is, the RofV, is increasing as the radius of the sphere increases. Indeed, the rate of increase in the RofV is

$$
\frac{\partial R o f V(r)}{\partial r}=2 r\left(\lambda_{k}-\lambda_{1}\right)>0
$$

for $\lambda_{k}>\lambda_{1}$ and $r>0$. The derivative is equal to 0 if and only if $r=0$ when $\lambda_{k}>\lambda_{1}$.

Case II : A first order model; the design center and region center are not the same.

The maximum and minimum prediction variances on a sphere of radius $r$ in this case are given by

$$
\max _{\underline{x} \in U_{r}} \frac{1}{\sigma^{2}} \operatorname{Var}(\hat{y}(\underline{x}))=\frac{1}{N}+\sum_{i=1}^{k} \lambda_{i}\left(\frac{\lambda_{i} m_{i}}{\lambda_{i}-\mu_{\max }}\right)^{2}
$$

where $\mu_{\max }>\lambda_{k}$ and $\sum_{i=1}^{k}\left(\frac{\lambda_{i} m_{i}}{\lambda_{i}-\mu_{\max }}\right)^{2}=r^{2}$, and

$$
\min _{\underline{x} \in U_{r}} \frac{1}{\sigma^{2}} \operatorname{Var}(\hat{y}(\underline{x}))=\frac{1}{N}+\sum_{i=1}^{k} \lambda_{i}\left(\frac{\lambda_{i} m_{i}}{\lambda_{l}-\mu_{\min }}\right)^{2}
$$

where $\mu_{\min }<\lambda_{1}$ and $\sum_{i=1}^{k}\left(\frac{\lambda_{i} m_{i}}{\lambda_{i}-\mu_{\min }}\right)^{2}=r^{2}$. They are functions of the radius, $r$, through the Lagrangian multipliers $\mu_{\max }$ and $\mu_{\min }$. Therefore, before considering the nature of the maximum and minimum prediction variance curves it is necessary to determine the relationship between the radius, $r$, and $\mu_{\max }, \mu_{\min }$. 
Let $\mu$ denote the Lagrangian multiplier for the prediction variance optimization problem described by (3.2.9). The multiplier $\mu$ defines a stationary point of the prediction variance on the surface of a sphere of radius $r$ if

$$
\sum_{i=1}^{k}\left(\frac{\lambda_{i} m_{i}}{\lambda_{i}-\mu}\right)^{2}=r^{2}
$$

If $\mu=\mu_{\max }>\lambda_{k}$ the stationary point is the point of maximum prediction variance on the sphere of radius $r$. The stationary point is the point of minimum prediction variance on the sphere if $\mu=\mu_{\min }<\lambda_{1}$. The nature of the relationship between $r$ and $\mu_{\max }, \mu_{\min }$ is sketched in Figure 14 . The convexity of the curve between succeeding values of the eigenvalues was first established by Draper (1963).

The following lemma is necessary to establish the trends in the maximum and minimum prediction variance functions as $r$ increases. The proof of the lemma is given in appendix B.1.

Lemma 4.1.1: Let $r>0$ and $\mu$ be defined by (4.1.3).

i) If $\mu=\mu_{\max }>\lambda_{k}$, as $r$ increases from 0 to $\infty$, $\mu_{\max }$ decreases. That is,

$$
\frac{\partial \mu_{\max }}{\partial r}<0 \quad \text { for } \mu_{\max }>\lambda_{k}
$$

ii) If $\mu=\mu_{\min }<\lambda_{1}$, as $r$ increases from 0 to $\infty$, $\mu_{\min }$ increases. That is,

$$
\frac{\partial \mu_{\min }}{\partial r}>0 \quad \text { for } \mu_{\min }<\lambda_{1}
$$




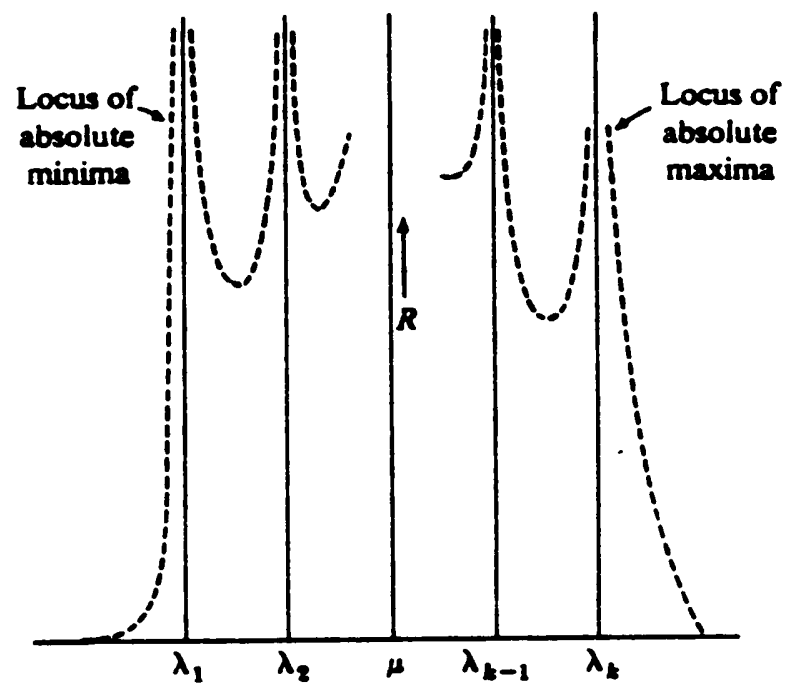

FIGORE 14.

PLOT OF \& KGIIIST

Reprodrecel vith peraission Ir "Respomse Surface Hethodolog"

by R.H. Hyers, Virginio Polytechaic lastitute and State Uaiversity, (1976). 
Consider now the maximum prediction variance curve defined by (4.1.1). By Lemma 4.1.1, the distance between $\mu_{\max }$ and any eigenvalue, $\lambda_{i}$, decreases as $r$ increases. Consequently, an increase in $r$ yields an increase in $\left[\lambda_{i}^{3} m_{i}^{2} /\left(\lambda_{i}-\mu_{\max }\right)^{2}\right]$ for each $\mathrm{i}=1,2, \ldots, \mathrm{k}$ and, therefore, in their sum. A similar argument gives that $\sum_{i=1}^{k}\left[\lambda_{i}^{3} m_{i}^{2} /\left(\lambda_{i}-\mu_{\min }\right)^{2}\right]$ increases as $r$ increases. These results lead to the following theorem.

Theorem 4.1.1: Under the conditions of case II,

i) the maximum prediction variance on a sphere of radius $r$ given by (4.1.1) is an increasing function of the radius;

ii) the minimum prediction variance on a sphere of radius $r$ given by (4.1.2) is an increasing function of the radius.

Proof of i): Let $Q=\sum_{i=1}^{k} \lambda_{i}\left(\frac{\lambda_{i} m_{i}}{\lambda_{i}-\mu_{\max }}\right)^{2}$. Then,

$$
\frac{\partial Q}{\partial r}=\frac{\partial Q}{\partial \mu_{\max }} \frac{\partial \mu_{\max }}{\partial r}=2 \sum_{i=1}^{k} \lambda_{i}^{3} m_{i}^{2}\left(\frac{1}{\lambda_{i}-\mu_{\max }}\right)^{3}\left[\frac{\partial \mu_{\max }}{\partial r}\right]
$$

Since $\mu_{\max }>\lambda_{k} \geq \lambda_{k-1} \ldots \geq \lambda_{1}>0,\left(\lambda_{i}-\mu_{\max }\right)<0$ for all $i=1,2, \ldots, k$. Hence, $\sum_{i=1}^{k} \lambda_{i}^{3} m_{i}^{2}\left(\lambda_{i}-\mu_{\max }\right)^{-3}<0$.

By Lemma 4.1.1, (i), $\frac{\partial \mu_{\max }}{\partial r}<0$ for $\mu_{\max }>\lambda_{k}$. Thus, $\frac{\partial Q}{\partial r}>0$ and the maximum prediction variance, $\frac{1}{N}+Q$, is an increasing function of $r$.

The proof of ii) is similar and will not be presented here.

In both case I and case II the maximum and minimum prediction variances and the range of the prediction variances on a sphere get larger as the radius of the sphere increases. The estimation of the response is less precise in general as one predicts further from the region center. When the design and region centers are the same, the variances of the predicted responses at locations of equal distance from the region center vary more widely as the distance increases. How- 
ever, it is believed that the RofV may not always be increasing in the radius $r$ when the region and design centers are at different locations. The trend in the RofV in this case probably depends on the distance between the design and region centers.

\subsubsection{Trends in the VofV as $r$ Increases}

The form of the variance of the prediction variances at locations on a sphere of radius $r$ is given by (3.2.15) and reproduced below for the first order model case.

$$
V o f V(r)=\frac{2 r^{4}}{k(k+2)} \sum_{i=1}^{k}\left(\lambda_{i}-\bar{\lambda}\right)^{2}+4 \frac{r^{2}}{k} \sum_{i=1}^{k} \lambda_{i}^{2} m_{i}^{2}
$$

This formulation of the VofV applies to case I as well as case II. Therefore, case I and case II will be considered concurrently.

It is obvious that as $r$ increases the $\operatorname{VofV}(r)$ will also increase. The rate at which $\operatorname{VofV}(r)$ increases is

$$
\frac{\partial V o f V(r)}{\partial r}=\frac{8 r^{3}}{k(k+2)} \sum_{i=1}^{k}\left(\lambda_{i}-\bar{\lambda}\right)^{2}+\frac{8 r}{k} \sum_{i=1}^{k} \lambda_{i}^{2} m_{i}^{2}
$$

Note that the amount of the increase in $\operatorname{VofV}(r)$ per unit increase in $r$ depends not only on the dispersion in the eigenvalues but also on the distance of the design center from the center of the region of the independent variables. The dispersion in the prediction variances will not increase as quickly if the design is centered at the center of the region under consideration.

Whether the experimental design can be classified under case I or case II, the quality of the estimated responses on spheres worsens as one predicts further from the center of the region. This can be seen from the increases in the $V^{r}$ and the maximum and minimum prediction variances as the radius of the sphere increases. The results of this section also show that variances corresponding 
to predicted responses on the surface of a sphere about the region center become more dispersed, that is, prediction is less consistent, as the radius of the sphere increases. The extent of the depreciation of the prediction capability of the design as the response is estimated further from the region center depends on the size and similarity of the eigenvalues $\lambda_{1}, \lambda_{2}, \ldots, \lambda_{k}$. Among competing designs, the smallest increases in the PVD measures would occur with the design which has the least deviation among the eigenvalues. To avoid large increases in the spherical variance and maximum and minimum prediction variances, a design with small as well as similar eigenvalues should be used.

\subsection{Invariance Properties of the Spherical Variance and}

\section{PVD Measures}

Measures of the prediction capability of a design should not depend on the natural units of the independent variables involved. For this reason, the independent variables are often expressed in terms of design variables which are scale-free. The choice of center and scale factors for the design variables is arbitrary. For example, the independent variables may be coded to be between -1 and +1 or to take values between -2 and +2 or 0 and 3 .

Often in a response surface analysis the region of the design variables is moved in the search for the optimum response of the system. The prediction capability of a design, however, should not be effected by the application of that design to a different region. Thus, the pictures of prediction capability developed using the spherical variance and PVD measures should be the same for a particular design regardless of the coding of the independent variables.

In this section, it will be shown for the first and second order model cases that the spherical variance and PVD measures are invariant to the center and scale chosen for the design variables of 
the system. It will also be shown that they are invariant to orthogonal transformation of the independent variables. These invariance properties are established for the $V^{r}$ and PVD measures via the invariance of the prediction variance to the units of the design variables. Further, sufficient conditions for the RofV and VofV to be invariant to the number center points of the design will be determined. Invariance to the number of center points is of particular interest in the consideration of the deviation from rotatability for a design.

\subsubsection{Invariance to the Scale of the Design Variables}

Consider a design, with corresponding design matrix $D_{x}$, used to fit the model $y=X \underline{\underline{Z}}+\underline{\varepsilon}$ in $\mathrm{k}$ design variables, $x_{1}, x_{2}, \ldots, x_{k}$. Without loss of generality, let the design variables be such that the center of the region of the $x$-variables is $x=0$ and the scale of the $x$-variables is equal to 1 for all $x_{i}$. The variance of a predicted value in terms of the $x$-variables is

$$
\operatorname{Var}_{D_{x}}(\hat{y}(\underline{x}))=\sigma^{2} \underline{x}^{*}\left(X^{\prime} X\right)^{-1} \underline{x}^{*}
$$

where $X$ is the appropriate model-matrix and $\underline{x}^{\circ}$ is as described in section 3.2 .3 for a first and second order model. The nature of $X$ and $\underline{x}^{*}$ depends on the model.

Suppose $z_{1}, z_{2}, \ldots, z_{k}$ are a different set of design variables to be used in the investigation of the response. Suppose that the region of the $z$-variables is centered at $\underline{z}=\underline{0}$ and the scale of the $z$-variables is equal to $q>0$, different from the scale of the $x$-variables. In this case, the $z$-variables can be written in terms of the $x$-variables as $z_{i}=q x_{i}$ for $i=1,2, \ldots, k$. The design matrix in the scaled $z$-variables corresponding to $D_{x}$ is thus,

$$
D_{z}=D_{x} Q
$$


for $Q=\operatorname{diagonal}(q, q, \ldots, q)$. Note that $Q$ is a kxk non-singular diagonal matrix. In terms of the scaled variables the model takes the form $y=Z_{\underline{Y}}+\underline{\varepsilon}$. The prediction variance at a location $\underline{z}=\left(\begin{array}{lllll}z_{1} & z_{2} & \ldots & z_{k}\end{array}\right)^{\prime}=Q \underline{x}$ corresponding to the model in the scaled variables is

$$
\operatorname{Var}_{D_{z}}(\hat{y}(\underline{z}))=\sigma^{2} \underline{z}^{*}\left(Z^{\prime} Z\right)^{-1} \underline{z}^{*}
$$

Here $Z$, is the appropriate model-matrix in terms of the $z$-variables. $Z$ and $\underline{z}^{*}$ depend on the model. In particular, if the model under consideration is a polynomial in the independent variables,

$$
Z=X Q^{*}
$$

where $X$ is the model-matrix in the original design variables and

$$
\underline{z}^{*}=Q^{*} \underline{x}^{*}
$$

The matrix $Q^{*}$ is diagonal and non-singular. If the model is a first order polynomial the modelmatrix $X$ is given by (2.2.2), $\underline{x}^{*}=\left(\begin{array}{lllll}1 & x_{1} & x_{2} & \ldots & x_{k}\end{array}\right)^{\prime}$ and

$$
Q^{*}=\left[\begin{array}{ccccc}
1 & 0 & 0 & \ldots & 0 \\
0 & q & 0 & \ldots & 0 \\
0 & 0 & q & \ldots & 0 \\
\cdot & . & . & . & \cdot \\
\cdot & . & . & . & \cdot \\
\cdot & . & . & . & \cdot \\
0 & 0 & 0 & \ldots & q
\end{array}\right]=\operatorname{diagonal}\left(\begin{array}{lllll}
1 & q & q & \ldots & q
\end{array}\right) .
$$

Alternately, if the model is a second order model in the independent variables the model matrix $X$ and vector $x^{*}$ are defined under case III in section 3.2.3. In this case, the matrix $Q^{*}$ is given by

$$
Q^{*}=\operatorname{diagonal}\left(\begin{array}{lllllllllll}
1 & q & \ldots & q & q^{2} & \ldots & q^{2} & q^{2} & \ldots & q^{2}
\end{array}\right)
$$


The form of $Q^{*}$ is easily extended to higher order models.

In this situation, it is apparent that due to the difference in scaling of the design variables the predicted response at a location $\underline{x}$ achieved by the analysis using the $x$-variables will not be the same as the predicted response at $\underline{z}=Q \underline{x}$ obtained by the analysis in the $z$-variables. However, the variances of these predicted responses will be the same. The following theorem establishes that under the circumstances described above the prediction variance at any location does not depend on the scale of the design variables.

Theorem 4.2.1: Suppose a polynomial model of order $d(d=1$ or 2 here $)$ in $k$ variables is used to approximate the response of a system. The prediction variance is invariant to the scale of the design variables of the model. Using the notation set forth above, this is equivalent to

$$
\left.\frac{1}{\sigma^{2}} \operatorname{Var}_{D_{x}} \hat{\hat{y}}(\underline{x})\right)=\frac{1}{\sigma^{2}} \operatorname{Var}_{D_{z}}(\hat{y}(\underline{z}))
$$

for any $\underline{x}$ in the space of the original $x$-design variables and $z=Q \underline{x} . \quad \operatorname{Var}_{D_{x}}$ and $\operatorname{Var}_{D_{z}}$ are given by (4.2.1) and (4.2.2).

Proof: Consider the models $\underline{y}=X \beta+\varepsilon$ and $y=Z_{\underline{\gamma}}+\varepsilon$ as described in the previous discussion. Consider the estimation of the response at an arbitrary location $\underline{x}$ in the space of the $x$-variables. The prediction variance at this location is given by (4.2.1). Prediction at $\underline{x}$ in the $x$-variables is equivalent to prediction at $\underline{z}=Q \underline{x}$ in the scaled variables. The prediction variance at $\underline{z}=Q \underline{x}$ is 


$$
\begin{aligned}
\frac{1}{\sigma^{2}} \operatorname{Var}_{D_{z}}(\hat{y}(\underline{z})) & =\underline{z}^{*}\left(Z^{\prime} Z\right)^{-1} \underline{z}^{*} \\
& =\underline{x}^{*} Q^{*}\left(Q^{*} X^{\prime} X Q^{*}\right)^{-1} Q^{*} \underline{x}^{*} \\
& =\underline{x}^{*}\left(X^{\prime} X\right)^{-1} \underline{x}^{*} \\
& =\frac{1}{\sigma^{2}} \operatorname{Var}_{D_{x}}(\hat{y}(\underline{x}))
\end{aligned}
$$

by (4.2.3) and (4.2.4) and since $Q^{\bullet}$ is symmetric and non-singular. The matrix $Q^{\bullet}$ is given by (4.2.5) when $d=1$ and (4.2.6) when the model is second order. Since the location $\underline{x}$ was arbitrarily chosen, the theorem is proved.

Consider now the prediction variances on a sphere of radius $r_{x}$ in the region of the $x$-variables. Let $U_{r_{x}}=\left\{\underline{x}: \sum_{i=1}^{k} x_{i}^{2}=r_{x}^{2}\right\}$ denote the surface of the sphere. Let $U_{r_{z}}=\left\{\underline{z}: \sum_{i=1}^{k} z_{i}^{2}=q^{2} \sum_{i=1}^{k} x_{i}^{2}=r_{z}^{2}=(q r)^{2}\right\}$ denote the surface of the corresponding sphere in the scaled variables. Clearly, if $\underline{x} \in U_{r_{x}}$ then $z=Q \underline{x} \in U_{r_{z}}$. Since $Q$ is a one-to-one transformation of $\underline{x}$ into $z$ there is only one location $\underline{x} \in U_{r_{x}}$ and one $z \in U_{r_{z}}$ for which $z=Q \underline{x}$. Thus, $Q$ provides a one-to-one mapping of $U_{r_{x}}$ into $U_{r_{z}}$.

By Theorem 4.2.1, for any location on the surface of a hypersphere of radius $r$ in the region of the $x$-variables defined by $U_{r_{x}}$, the prediction variance there is the same as the prediction variance at the corresponding location on $U_{r_{z}}$. Therefore, the prediction variances at all locations on $U_{r_{x}}$ are the same as those on $U_{r_{z}}$. As a consequence, the average prediction variance, the maximum and minimum prediction variances and the dispersion in the prediction variances on a sphere will be the same whether the $x$-design variables or the $z$-design variables are used. Thus, the $V^{r}, \max$ imum and minimum prediction variances and the RofV and VofV are invariant to the scale of the design variables used in the analysis. 


\subsubsection{Invariance to the Center of the Design Variables}

Once again consider a system involving $\mathrm{k}$ independent variables. Suppose the variables are coded to the design variables $x_{1}, x_{2}, \ldots, x_{k}$ through a simple linear transformation such as that described by (2.2.1). Although it is usual to code the independent variables to have center at $\underline{x}=\underline{Q}$, this is not always the case. Let $\underline{h}=\left(\begin{array}{llll}h_{1} & h_{2} & \ldots & h_{k}\end{array}\right)^{\prime}$ denote the center of the region of the design variables in this situation. For convenience and without loss of generality, suppose $\underline{h}=\underline{0}$.

Let $z_{1}, z_{2}, \ldots, z_{k}$ represent a different coding of the independent variables for which $z_{i}=x_{i}+m_{i}$ for some $m_{i}$ and $i=1,2, \ldots, k$. The new coding of the variables is equivalent to a translation of the axes in the $\mathrm{k}$-dimensional region of the $x$-variables to the $\mathrm{k}$-dimensional region of the $z$-variables. It is of interest to compare the prediction variances on spheres about the different centers of the region of the $x$-variables and the region of the $z$-variables for a design.

In terms of the $x$-design variables the prediction variance at a $x$ is

$$
\operatorname{Var}_{D_{x}}(\hat{y}(\underline{x}))=\sigma^{2} \underline{x}^{*}\left(X^{\prime} X\right)^{-1} \underline{x}^{*}
$$

The vector $\underline{x}^{*}$ and model-matrix $X$ are defined as before for a first and second order model, (see section 3.2.3). The subscript $D_{x}$ indicates that the $x$-variables were used to compute the variance. The prediction variance at a location $\underline{z}=\underline{x}+\underline{m}$ in the space of the $z$-variables is given by

$$
\operatorname{Var}_{D_{z}}(\hat{y}(z))=\sigma^{2} \underline{z}^{*}\left(Z^{\prime} Z\right)^{-1} z^{*}
$$

for appropriate model-matrix $Z$ and vector $\underline{z}^{\circ}$.

The following lemma will be necessary to establish the invariance of the prediction variance to the center of the design variables for the first and second order model cases.

Lemma 4.2.1: Consider fitting a polynomial model of first or second order to the response 
of a system in $\mathrm{k}$ variables with a design. Let the design variables $x_{1}, x_{2}, \ldots, x_{k}$ and $z_{1}, z_{2}, \ldots, z_{k}$ be as defined in the previous discussion. Then, there exists a pxp matrix $L$ of rank $\mathrm{p}$ for which

$$
Z=X L
$$

where $\mathrm{p}$ is the number of parameters in the model and $Z$ and $X$ are the appropriate model-matrices in the $z$-variables and $x$-variables respectively. Also, $\underline{z}^{*}=L^{\prime} \underline{x}^{*}$.

Proof: The proof Lemma 4.2.1 appears in appendix B.2. Forms for the matrix $L$ in the first and second order model cases are given there also.

As a consequence of Lemma 4.2.1 the following result is obtained.

Theorem 4.2.2: For the case of fitting a first or second order model to the response of a system with design variables the prediction variance is invariant to the center of the design variables used in the analysis.

Proof: It is necessary to show that

$$
\frac{1}{\sigma^{2}} \operatorname{Var}_{D_{x}}(\hat{y}(\underline{x}))=\frac{1}{\sigma^{2}} \operatorname{Var}_{D_{z}}(\hat{y}(z))
$$

for any $\underline{x}$ in the region of the $x$-variables and corresponding $z=\underline{x}+\underline{m}$ where $\underline{m}=\left(\begin{array}{lllll}m_{1} & m_{2} & \ldots & m_{k}\end{array}\right)^{\prime}$.

Consider a location $\underline{x}$ in the region of the $x$-variables. By definition, the prediction variance at the corresponding location, $z$ in the space of the $z$-variables is

$$
\operatorname{Var}_{D_{z}}(\hat{y}(z))=\sigma^{2} z^{*}\left(Z^{\prime} Z\right)^{-1} \underline{z}^{*}
$$

By Lemma 4.2.1, $Z=X L$ and $\underline{z}^{*}=L^{\prime} \underline{x}^{*}$. Thus, 


$$
\begin{aligned}
\frac{1}{\sigma^{2}} \operatorname{Var}_{D_{z}}(\hat{y}(\underline{z})) & =\underline{x}^{*} L\left(L^{\prime} X^{\prime} X L\right)^{-1} L^{\prime} \underline{x}^{*} \\
& =\underline{x}^{*} L(L)^{-1}\left(X^{\prime} X\right)^{-1}\left(L^{\prime}\right)^{-1} L^{\prime} \underline{x}^{*} \\
& =\underline{x}^{*}\left(X^{\prime} X\right)^{-1} \underline{x}^{*} \\
& \left.=\frac{1}{\sigma^{2}} \operatorname{Var}_{D_{x}} \hat{y}(\underline{x})\right) .
\end{aligned}
$$

Similarly, for any location $\underline{z}$ in the region of the $z$-variables, $\underline{x}=\underline{z}-\underline{m}$ and $\operatorname{Var}_{D_{z}}(\hat{y}(\underline{z})) / \sigma^{2}=\operatorname{Var}_{D_{x}}(\hat{\dot{y}}(\underline{x})) / \sigma^{2}$

This holds for any $\underline{x}, \underline{z}$ and any $\underline{m}$, so the prediction variance does not depend on the center of the design variables. In other words, the prediction variance is invariant to the center of the region of the design variables.

Now return to the consideration of the prediction capability on spheres for a design. Any location $\underline{x}$ on the surface of a sphere of radius $r$ about the center of the region of the design variables $x_{1}, \ldots x_{k}$ has one and only one corresponding location in the region of the $z$-variables. The corresponding $z=\underline{x}+\underline{m}$ is located on the surface of a sphere of radius $r$ about the region center of the $z$-variables. The prediction variances are the same at corresponding locations by Theorem 4.2.2. Therefore, the prediction variances expressed in design variables on the surface of a hypersphere of radius $r$ are invariant to the location of the center of the sphere. It is intuitively obvious then, that the spherical variance, maximum and minimum prediction variances and the PVD measures are not effected by the position of the center of the region of the design variables in $\mathrm{k}$-dimensional space. 


\subsubsection{Invariance to Orthogonal Transformation of the Independent Variables}

The optimization of the response of a system usually requires an extensive search in the region of the independent variables. The researcher begins the search for the optimum response by modelling the response as a simple function of the factors present in the system in a suitable region of the independent variables. Based on the information acquired at this stage, the model may be updated to a more complicated form, or the region of search extended or shifted or both.

For instance, if a stationary point has been obtained in some region, the region may be shifted to a new region with center at the stationary point. This action facilitates the determination of the nature of the stationary point. It has already been seen that the picture of prediction capability for a design is not effected by a translation of the region of the design variables.

To further aid in the determination of the status of the stationary point and the behavior of the response about it, a canonical analysis of the response function is performed. In a canonical analysis the response function is expressed in terms of the principal component axes of the new region. That is, the axes in the new region corresponding to the design variables are rotated through the origin by an orthogonal transformation to the principal components of the system.

It is of interest to determine the effect of an orthogonal transformation of the design variables, such as would occur in a canonical analysis, on the spherical variance and PVD measures for a design. Let the response function be expressed in terms of the variables $x_{1}, x_{2}, \ldots, x_{k}$ in some region. It is not necessary that $x_{1}, x_{2}, \ldots, x_{k}$ be design variables. Let $D_{x}$ represent the design matrix in terms of the $x$-variables.

Now consider an orthogonal transformation of the $x$-variables to a new axis system in the variables $z_{1}, z_{2}, \ldots, z_{k}$ as follows: 


$$
\begin{aligned}
\underline{z} & =\left(\begin{array}{llll}
z_{1} & z_{2} & \ldots & z_{k}
\end{array}\right)^{\prime} \\
& =R \underline{x}=R\left(\begin{array}{lllll}
x_{1} & x_{2} & \ldots & x_{k}
\end{array}\right)^{\prime}
\end{aligned}
$$

where $R$ is an orthogonal kxk matrix. The resulting axis system in the $z$-variables is a rotation through the origin of the axis system in the $x$-variables.

The result that the prediction variance at a location remains unchanged by the rotation of the region through its origin is proved in the next theorem for polynomial models.

Theorem 4.2.3: Suppose the model for the response is a polynomial of order $\mathrm{d}$ in $\mathrm{k}$ variables.

The variance of an estimated response obtained with a design is invariant to the orthogonal transformation of the independent variables.

Proof: Let $x_{1}, x_{2}, \ldots, x_{k}$ denote the variables of the model and $D_{x}$ the design matrix in terms of the $x$-variables. Then, $\operatorname{Var}_{D_{x}}(\hat{y}(\underline{x})) / \sigma^{2}=\underline{x}^{\prime \prime}\left(X^{\prime} X\right)^{-1} \underline{x}^{*}$, where $\underline{x}^{*}$ and $X$ have their usual meaning.

Suppose $\underline{x}=\left(\begin{array}{lllll}x_{1} & x_{2} & \ldots & x_{k}\end{array}\right)^{\prime}$ is transformed by an orthogonal matrix $R$ to $\underline{z}=\left(\begin{array}{lllll}z_{1} & z_{2} & \ldots & z_{k}\end{array}\right)^{\prime}$. That is, $\underline{z}=R \underline{x}$. Let $D_{z}$ denote the design matrix expressed in the $z$-variables, then $D_{z}=D_{x} R^{\prime}$. Now, $\operatorname{Var}_{D_{z}}(\hat{y}(z)) / \sigma^{2}=\underline{z}^{* \prime}\left(Z^{\prime} Z\right)^{-1} z^{*}$, where $\underline{z}^{*}$ and $Z$ have their usual meaning.

There exists an orthogonal matrix, say $R^{*}$, for which

$$
\begin{aligned}
& \underline{z}^{*}=R^{*} \underline{x}^{*} \text { and } \\
& Z=X R^{*},
\end{aligned}
$$

$R^{*}$ is called the Schlaifflian matrix corresponding to the transformation matrix $R$. The reader is referred to Aitken (1948) or Myers ((1976), (appendix a.1)) for verification of this result. 
Since $R^{*}$ is an orthogonal matrix, its inverse exists and is equal to $R^{\cdot-1}=R^{\prime \prime}$. Equivalently, $\left(R^{* \prime}\right)^{-1}=R^{*}$. So, the prediction variance in terms of the $z$-variables can be written as

$$
\begin{aligned}
\frac{1}{\sigma^{2}} \operatorname{Var}_{D_{z}}(\hat{y}(\underline{z})) & =\underline{x}^{* \prime} R^{* \prime}\left(R^{*} X^{\prime} X R^{* \prime}\right)^{-1} R^{*} \underline{x}^{*} \\
& =\underline{x}^{*} R^{*} R^{*}\left(X^{\prime} X\right)^{-1} R^{*} R^{*} \underline{x}^{*} \\
& =\underline{x}^{*}\left(X^{\prime} X\right)^{-1} \underline{x}^{*} \\
& =\frac{1}{\sigma^{2}} \operatorname{Var}_{D_{x}}(\hat{y}(\underline{x}))
\end{aligned}
$$

for $z=R \underline{x}$. Thus, for any location $\underline{x}$ the prediction variance at $\underline{x}$ is equal to the corresponding prediction variance in the region of the $z$-variables. That is, the prediction variance is invariant to the orthogonal transformation of the independent variables.

Note that, if $\underline{x} \in U_{r}=\left\{\underline{x}: \sum_{i=1}^{k} x_{i}^{2}=r^{2}\right\}$, the surface of a k-dimensional hypersphere of radius $r$, then $\underline{z}=R \underline{x}$ is also a member of $U_{r}$. Thus, the prediction variances on a sphere of radius $r$ about the center of region are the same under an orthogonal transformation of the $x$-variables. Since a location $\underline{x}$ on a sphere is taken into one and only one location $\underline{z}$ on that sphere by the orthogonal transformation, the average and maximum and minimum values of the prediction variances on a sphere also remain unchanged by orthogonal transformation of the variables. Similarly, the dispersion measures RofV and VofV are invariant to orthogonal transformation of the independent variables.

To summarize, the spherical variance, maximum and minimum prediction variances and the PVD measures are all invariant to the center and scale of the design variables chosen for the analysis. The orientation of the region of the variables in $\mathrm{k}$-dimensional space also has no bearing on the picture of the prediction capability of a design generated with these measures. Thus, once a 
picture of the prediction capability on spheres for a particular design has been developed in terms of a given set of design variables, it is not necessary to generate another picture for a different set of design variables.

\subsubsection{Invariance of RofV and VofV to the Number of Center Points of the} Design

When the center of the design and the center of the region of the design variables are the same, the RofV and VofV indicate how near the design is to possessing the rotatability property. The equality of variances on spheres about the design center is unaffected by variation of the number of experimental runs taken at the center of the design in a rotatable design. Thus, it is of interest to determine if the RofV and VofV, as measures of deviation from rotatability, are invariant to the number of center points, $n_{0}$, of the design. In this section, sufficient conditions for the PVD measures to be invariant to $n_{0}$ will be given for the cases of fitting a first and second order model to the response of the system.

Consider a design in $\mathrm{k}$ variables of size $N=n+n_{0}$, where $n_{0}$ is the number of center points of the design. In this discussion, the region of the design variables will be considered to have center where all the design variables take the value 0 . A center point is a point for which the levels of all the design variables are at 0 . The prediction variances obtained with the design depends on the design settings through the $\left(X^{\prime} X\right)$ matrix, or, more specifically, its inverse.

Suppose, first, that the design is to be used to fit a first order model to the response. Let the design variables be denoted by $x_{1}, x_{2}, \ldots, x_{k}$. In this situation, the $X^{\prime} X$ matrix has the form given below. 


$$
X^{\prime} X=\left[\begin{array}{ll}
N & \mathcal{c}^{\prime} \\
\mathcal{c} & A
\end{array}\right]
$$

where $N=n+n_{0}, \quad c=\left(\sum_{u=1}^{N} x_{u 1} \sum_{u=1}^{N} x_{u 2} \ldots \sum_{u=1}^{N} x_{u k}\right)^{\prime}$ and

$$
A=\left[\begin{array}{cccc}
\sum x_{u 1}^{2} & \sum x_{u 1} x_{u 2} & \ldots & \sum x_{u 1} x_{u k} \\
\sum x_{u 1} x_{u 2} & \sum x_{u 2}^{2} & \ldots & \sum x_{u 2} x_{u k} \\
\cdot & \cdot & \cdot & \cdot \\
\cdot & \cdot & \cdot & \cdot \\
\cdot & \cdot & \cdot & \cdot \\
\Sigma x_{u 1} x_{u k} & \sum x_{u 2} x_{u k} & \ldots & \Sigma x_{u k}^{2}
\end{array}\right]
$$

Note that $\varsigma / N$ is the location of the center of the design.

The prediction variance at a location $\underline{x}=\left(\begin{array}{llll}x_{1} & x_{2} & \ldots & x_{k}\end{array}\right)^{\prime}$ with $\underline{x}^{*}=\left(\begin{array}{llll}1 & x_{1} & x_{2} & \ldots \\ x_{k}\end{array}\right)^{\prime}$ for a first order model is

$$
\begin{aligned}
\frac{1}{\sigma^{2}} \operatorname{Var}(\hat{y}(\underline{x}))= & \underline{x}^{*}\left(X^{\prime} X\right)^{-1} \underline{x}^{*} \\
= & {\left[N-\underline{c}^{\prime} A^{-1} \underline{c}\right]^{-1}-\frac{2}{N}\left[\underline{x}^{\prime} A^{-1} \underline{\varepsilon}+\frac{\underline{x}^{\prime} A^{-1} \underline{c}^{\prime} A^{-1} \underline{c}}{N-\underline{c}^{\prime} A^{-1} \underline{c}}\right] } \\
& +\left[\underline{x}^{\prime} A^{-1} \underline{x}+\frac{\underline{x}^{\prime} A^{-1} \underline{c} \underline{c}^{\prime} A^{-1} \underline{x}}{N-\underline{c}^{\prime} A^{-1} \underline{c}}\right]
\end{aligned}
$$

The formulation of this result requires the use of the inverse of a pattern matrix and matrix algebra. It will not be reproduced here.

A condition for which the RofV and VofV are invariant to the number of center points of the design in the first order model case is presented in Theorem 4.2.4. 
Theorem 4.2.4: Suppose a first order model and design of size $N=n+n_{0}$ are used to estimate the response of a system. Let $x_{1}, x_{2}, \ldots, x_{k}$ denote the design variables in this situation. Then, the RofV and VofV are invariant to the number of center points, $n_{0}$, of the design if the design center is located at the center of the region of the design variables. This condition is equivalent to the requirement that the first design moments of the design be equal to zero, i.e.,

$$
[\mathrm{i}]=\frac{1}{N} \sum_{u=1}^{N} x_{u i}=0 \text { for all } \mathrm{i}=1,2, \ldots, \mathrm{k} \text {. }
$$

(See section 2.3.4 for a description of design moments.)

Proof: The proof is presented for the RofV. The result for the VofV is similarly proved and will not be shown here.

Suppose the center of the region of the design variables is at $\underline{x}=\underline{0}$. Also, suppose the center of the design is located at $\underline{x}=\underline{0}$, the region center. This implies that,

$$
c=\left(\sum_{u=1}^{N} x_{u 1} \sum_{u=1}^{N} x_{u 2} \ldots \sum_{u=1}^{N} x_{u k}\right)^{\prime}=0
$$

Under this condition, the prediction variance given by $(4.2 .9)$ reduces to

$$
\frac{1}{\sigma^{2}} \operatorname{Var}(\hat{y}(\underline{x}))=\frac{1}{N}+\underline{x}^{\prime} A^{-1} \underline{x}
$$

Let $\underline{x}_{\max }$ and $\underline{x}_{\min }$ represent the locations of the maximum and minimum prediction variances respectively on the surface of a hypersphere of radius $r$. Then, the range of the prediction variances on the sphere is

$$
R o f V(r)=\underline{x}_{\max } A^{-1} \underline{x}_{\max }-\underline{x}_{\min }^{\prime} A^{-1} \underline{x}_{\min } .
$$


The elements of the matrix $A$ and, hence, those of $A^{-1}$, do not depend on the value of $n_{0}$. Therefore, the RofV does not depend on $n_{0}$ in this case. That is, if the design center is the same as the region center, the RofV is invariant to the number of center points of the design.

In the second order model case, the conditions for the invariance of RofV and VofV to the number of center points involve not only the first order moments, but all design moments through order four of the design. The conditions are stated in the following theorem.

Theorem 4.2.5: Suppose a second order model and design of size $N=n+n_{0}$ are used to estimate the response of a system. Let $x_{1}, x_{2}, \ldots, x_{k}$ denote the design variables in this situation. Then, the RofV and VofV are invariant to the number of center points of the design if the following conditions hold:

i) All odd moments through order four are equal to zero, that is,

$$
\begin{aligned}
& {[\mathrm{i}]=0 \text { for all } \mathrm{i}=1,2, \ldots, \mathrm{k},} \\
& {[\mathrm{ij}]=0 \text { for all } \mathrm{i} \neq \mathrm{j},} \\
& {[\mathrm{iii}]=[\mathrm{iij}]=0 \text { for all } \mathrm{i} \neq \mathrm{j},} \\
& {[\mathrm{iiij}]=0 \text { for all } \mathrm{i} \neq \mathrm{j} .}
\end{aligned}
$$

ii) The second pure moments of the design are equal. That is,

$$
\text { [ii] }=a / N \text {, a constant for all } \mathrm{i}=1,2, \ldots, \mathrm{k} \text {. }
$$

iii) The fourth pure moments are equal. That is,

$$
\text { [iiii }]=b / N \text {, a constant for all } \mathrm{i}=1,2, \ldots, \mathrm{k} \text {. }
$$

iv) The even fourth mixed moments are equal. That is,

$$
[\mathrm{iijj}]=c / N \text {, a constant for all } \mathrm{i}, \mathrm{j}=1,2, \ldots, \mathrm{k} \text { with } \mathrm{i} \neq \mathrm{j} \text {. }
$$


Proof: The proof follows that of Theorem 4.2.4. It appears in appendix B.3.

Note that the conditions set forth in Theorems 4.2.4 and 4.2.5 are only sufficient conditions for the RofV and VofV to be invariant to $n_{0}$. Any rotatable design satisfies these conditions, thus, showing that the rotatability property is not altered by the addition of experimental runs at the center of the design. The dispersion in the prediction variances on spheres may be altered by the addition of center points if the non-rotatable design does not meet the requirements of Theorems 4.2.4 or 4.2.5.

\subsection{A New Criterion for Design Augmentation}

There is very little breadth in the area of design augmentation in the literature. Virtually all the results in this area pertain to the augmentation of a design to achieve a conditionally D-optimal design, (see, for example, Dykstra (1971), Hebble and Mitchell (1972) or Gaylor and Merrill (1968)). Recall that a conditionally D-optimal design in some region is achieved by augmenting the point in that region which yields the largest increase in $\left|X^{\prime} X\right|$.

This strategy results in the most improved estimation of coefficients of the model as measured by the generalized variance of the coefficients. Although the prediction capability of the design is naturally enhanced by the addition of a point to maximize $\left|X^{\prime} X\right|$, there is no evidence that this particular choice of an additional design point will yield the most improvement in the prediction capability of a design. Indeed, the addition of a point which directly improves the prediction variances, or some function of them, would most likely result in better improvement of the prediction capability of a design.

With a view to improving the precision of the estimated responses on spheres for a design, one might consider augmenting a point to the design in order to make the resulting spherical vari- 
ance on some sphere as small as possible. It is thought that the greatest improvement in the spherical variances across all spheres would result from the minimization of the spherical variance on the sphere corresponding to the largest $V^{r}$ for the original design. The minimization of the spherical variance is conditional on the settings of the original design.

Consider a design with $N$ design points to fit a model in $\mathrm{k}$ variables. Let $\mathrm{p}$ be the number of parameters in the model and $X$ be the model-matrix in this situation. The prediction variance at a location $\underline{x}=\left(\begin{array}{lllll}x_{1} & x_{2} & \ldots & x_{k}\end{array}\right)^{\prime}$ is given by $\operatorname{Var}(\hat{y}(\underline{x})) / \sigma^{2}=\underline{x}^{\prime \prime}\left(X^{\prime} X\right)^{-1} \underline{x}^{*}$ where the elements of $\underline{x}^{\cdot}$ depend on the model as before (see section 3.2.3). Let $V^{r}$ denote the spherical variance corresponding to this design and model. Let $\underline{x}_{a}$ denote the point which is to be added to the design. The model-matrix corresponding to the design augmented by $\underline{x}_{\alpha}$ is then,

$$
X_{a}=\left[\begin{array}{c}
X \\
x_{a}^{*},
\end{array}\right]
$$

where $\underline{x}_{a}^{*}$ has the same form as $\underline{x}^{*}$.

Letting $\hat{y}_{a}(\underline{x})$ represent the predicted response at $\underline{x}$ obtained with the augmented design, the prediction variance for the augmented design is

$$
\frac{1}{\sigma^{2}} \operatorname{Var}\left(\hat{y}_{a}(\underline{x})\right)=\underline{x}^{*}\left(X_{a}^{\prime} X_{a}\right)^{-1} \underline{x}^{*}=\underline{x}^{*}\left(X^{\prime} X+\underline{x}_{a}^{*} \underline{x}_{a}^{* \prime}\right)^{-1} \underline{x}^{*}
$$

The inverse is found by applying the Sherman-Morrison-Woodbury theorem (Rao (1973) problem 2.8, p. 33) which states that for a positive definite pxp matrix $A$ and pxl vector $\underline{x}_{0}$,

$$
\left(A+\underline{x}_{0} \underline{x}_{0}^{\prime}\right)^{-1}=A^{-1}-\frac{A^{-1} \underline{x}_{0} \underline{x}_{0}^{\prime} A^{-1}}{1+\underline{x}_{0}^{\prime} A^{-1} \underline{x}_{0}} .
$$

Thus, 


$$
\begin{aligned}
\frac{1}{\sigma^{2}} \operatorname{Var}\left(\hat{y}_{a}(\underline{x})\right) & =\underline{x}^{* \prime}\left(X^{\prime} X\right)^{-1} \underline{x}^{*}-\frac{\underline{x}^{*}\left(X^{\prime} X\right)^{-1} \underline{x}_{a}^{*} \underline{x}_{a}^{*}\left(X^{\prime} X\right)^{-1} \underline{x}^{*}}{1+\underline{x}_{a}^{*}\left(X^{\prime} X\right)^{-1} \underline{x}_{a}^{*}} \\
& =\frac{1}{\sigma^{2}} \operatorname{Var}(\hat{y}(\underline{x}))-\frac{\underline{x}^{*}\left(X^{\prime} X\right)^{-1} \underline{x}_{a}^{*} \underline{x}_{a}^{* \prime}\left(X^{\prime} X\right)^{-1} \underline{x}^{*}}{1+\underline{x}_{a}^{*}\left(X^{\prime} X\right)^{-1} \underline{x}_{a}^{*}}
\end{aligned}
$$

The spherical variance resulting from the augmentation of $\underline{x}_{a}$ to the design is given by $V_{a}^{r}$ below.

$$
\begin{aligned}
V_{a}^{r} & =\Psi \int_{U_{r}} \frac{1}{\sigma^{2}} \operatorname{Var}\left(\hat{y}_{a}(\underline{x})\right) d \underline{x} \\
& =V^{r}-\frac{\underline{x}_{a}^{*}\left(X^{\prime} X\right)^{-1} S\left(X^{\prime} X\right)^{-1} \underline{x}_{a}^{*}}{1+\underline{x}_{a}^{*}\left(X^{\prime} X\right)^{-1} \underline{x}_{a}^{*}}
\end{aligned}
$$

where $U_{r}=\left\{\underline{x}: \sum_{i=1}^{k} x_{i}^{2}=r^{2}\right\}$ represents the surface of a hypersphere of radius $r$ with surface area given by $\Psi^{-1}=\int_{U_{r}} d \underline{x}$. The matrix $S$ is the matrix of spherical region moments defined by (3.2.6) for the model. The derivation of this result very closely follows the derivation of the $V^{r}$ given in Hussey (1983).

The suggested criterion requires the point $\underline{x}_{\sigma}$ be chosen so that $V_{a}^{r}$ is minimized. Since the second term of the right hand side of $(4.3 .1)$ is nonnegative, this is equivalent to choosing $\underline{x}_{a}$ to maximize the quantity

$$
\frac{\underline{x}_{a}^{*}\left(X^{\prime} X\right)^{-1} S\left(X^{\prime} X\right)^{-1} \underline{x}_{a}^{*}}{1+\underline{x}_{a}^{*}\left(X^{\prime} X\right)^{-1} \underline{x}_{a}^{*}} .
$$

This result provides a new prospect for the development of a design augmentation strategy in response surface studies. To a researcher interested in predicting the response of a system, particularly on spheres, the spherical variance criterion may be more appealing than the conditional 
$\left|X^{\prime} X\right|$ criterion. Extensive case studies are needed to compare the prediction capabilities of augmented designs formed under the two criteria.

\subsection{The Dilemma over the Choice of Design Center}

It has already been seen that in the first order model case it is to the researcher's advantage to choose a design which is centered about the center of the region of interest. In this case, the spherical variance and the dispersion in the prediction variances on spheres are smaller when the design and region centers are the same. It is suspected that this statement holds for second order designs as well.

The improved prediction capability is a strong motivation for centering the design about the region center. However, centering may be foregone to achieve some other design property such as high D-efficiency. The dilemma arises over the decision to move the region of interest to coincide with the new design center after some design points have been lost or added during experimentation.

The region of interest is often shifted during the exploration of a response surface and search for the optimum response. It is the opinion of the author that the region of interest should not be changed to accommodate a change in the design caused by a loss of points or a poorly designed experiment. Rather, the design should be changed if the region under consideration is changed.

Ultimately, the choice of design center and region of interest belongs to the researcher. Whether the design center and region center have been chosen to be the same or not, the spherical variance and PVD measures are equipped to provide a picture of the prediction capability on spheres for the design in that region. 
For the illustrations of the use of the spherical variance and RofV to assess the quality of prediction for second order designs in Chapter V, the centers of the example designs have been left as originally specified by their authors. In the cases where design points have been lost, the region under consideration has not been changed to coincide with the new design region. Thus, comparisons between the original design and the designs resulting from lost data are made in the same region. 


\section{Chapter V}

\section{Graphical Assessment of the Prediction Capability of Certain Second Order Designs}

The experimental designs catalogued in section 2.4 represent various experimental plans the researcher may choose to explore a response surface. Some, such as the composite, Box-Behnken and hybrid designs, are often used in practice. Recently, there has been increasing interest in the minimum and nearly minimum point designs as economical alternatives to the larger response surface designs such as the central composite design (ccd). Most of the investigations into the design properties of saturated and nearly saturated designs has been concerned with the D-efficiencies of the designs (for example, Lucas (1976), Mitchell and Bayne (1978) and Nalimov, et. al. (1970)). Few studies have given attention to the quality of the predictions obtained with these designs.

It is the purpose of this chapter to examine through the use of graphics the prediction capabilities of some of the second order designs presented in section 2.4. General pictures of the behavior of the prediction variances throughout a region will be developed for the designs via the spherical variance and RofV functions. In some cases, the locations of the maximum and minimum prediction variances will be used to indicate at which combination of levels of the variables pre- 
diction quality is poorest and where it is best. Several sets of comparisons of competing designs will also be made. For example, comparisons between two ccds and the Box-Behnken design in three variables will be conducted in section 5.1.2. The use of the RofV to detect deviation from rotatability for a design will be discussed as a separate issue in the last section. First, however, the prediction capability of some members of the family of central composite designs will be examined. Specifically, the effect of the choice of the design parameters $\alpha$ and $n_{0}$ on the picture of the prediction capability for a ccd will be considered. Also, the loss of prediction capability will be assessed when one or more design points have been dropped from a rotatable ccd.

The experimental designs considered in this chapter are second order designs in three variables with the exception of the $3^{2}$ factorial design in section 5.3.1. The behavior of the prediction variances for the three variable designs will be investigated across a range of radii, from $r=0$, (representing prediction at the region center), to $r=\sqrt{3}=1.732$, (representing prediction at locations on the perimeter of the region of interest). Thus, the region of the design variables under

consideration is a hypersphere of radius $r=\sqrt{3}=1.732$ centered about the point $x_{1}=0, x_{2}=0, x_{3}=0$ in three dimensional space. All prediction variances have been weighted by $N$, the size of the design, unless otherwise noted.

\subsection{The Family of Central Composite Designs}

\subsubsection{Comparisons of Prediction Capability in Three Variable CCDs}

Central composite designs (section 2.4.2) are distinguished by the values of the design parameters $\alpha$ and $n_{0}$. The axial value, $\alpha$, may be chosen to achieve the rotatability property. A dif- 
ferent value may be required to achieve other design properties, for example, minimum integrated variance among all ccds of the same size.

The plots of spherical variance in Figure 15 provide a comparison of the average predicting ability of three ccds. Each of the three designs is the same, $2^{3}$ factorial portion plus one center point, except for the axial values. The design labelled ' 1 ' in Figure 15 is a rotatable ccd. The axial value is $\alpha=1.682$. Design 2 and design 3 have axial values of 1.35 and 1.0 , respectively. Note that a three variable ccd has its center at the point $x_{1}=0, x_{2}=0, x_{3}=0$, the region center.

The closer $\alpha$ is to the center of the region the better prediction is in an area about the center. Beyond a radius of about 1 the designs with larger axial values, that is, with more design points in this part of the region, provide more precise estimation of the response. The relatively low variances obtained with design 3 on spheres near the region center may be explained by the closeness of the axial points to the center of the region. Thus, the axial points, as well as the center points, provide information about the response near the center. The ccds with larger axial values mainly rely on the information provided by center runs to estimate the response near the center.

There is no dispersion in the prediction variances on spheres for the rotatable design, (design 1). Plots of the maximum and minimum prediction variance curves representing dispersion in the variances on spheres appear in Figure 16 for design 2 and Figure 17 for design 3. The dispersion in the variances for design 2 , with an axial value close to the rotatable value, is slight. For both designs the variances remain very stable on and within a sphere of radius 1 . On radii beyond the region encompassed by the axial points, $\left(\begin{array}{lll}\alpha & 0 & 0\end{array}\right),\left(\left(\begin{array}{lll}-\alpha & 0 & 0\end{array}\right)\right.$, etc, the third design with $\alpha=1.0$ yields less precise and more inconsistent estimation of the response than the designs with larger axial values.

It should be noted that the experimenter who chooses to use a ccd with $\alpha=1.0$ is most likely not interested in predicting the response on spheres beyond a radius of 1 . Rather, the experimenter probably wishes to predict only at locations for which the levels of the design variables are no less 


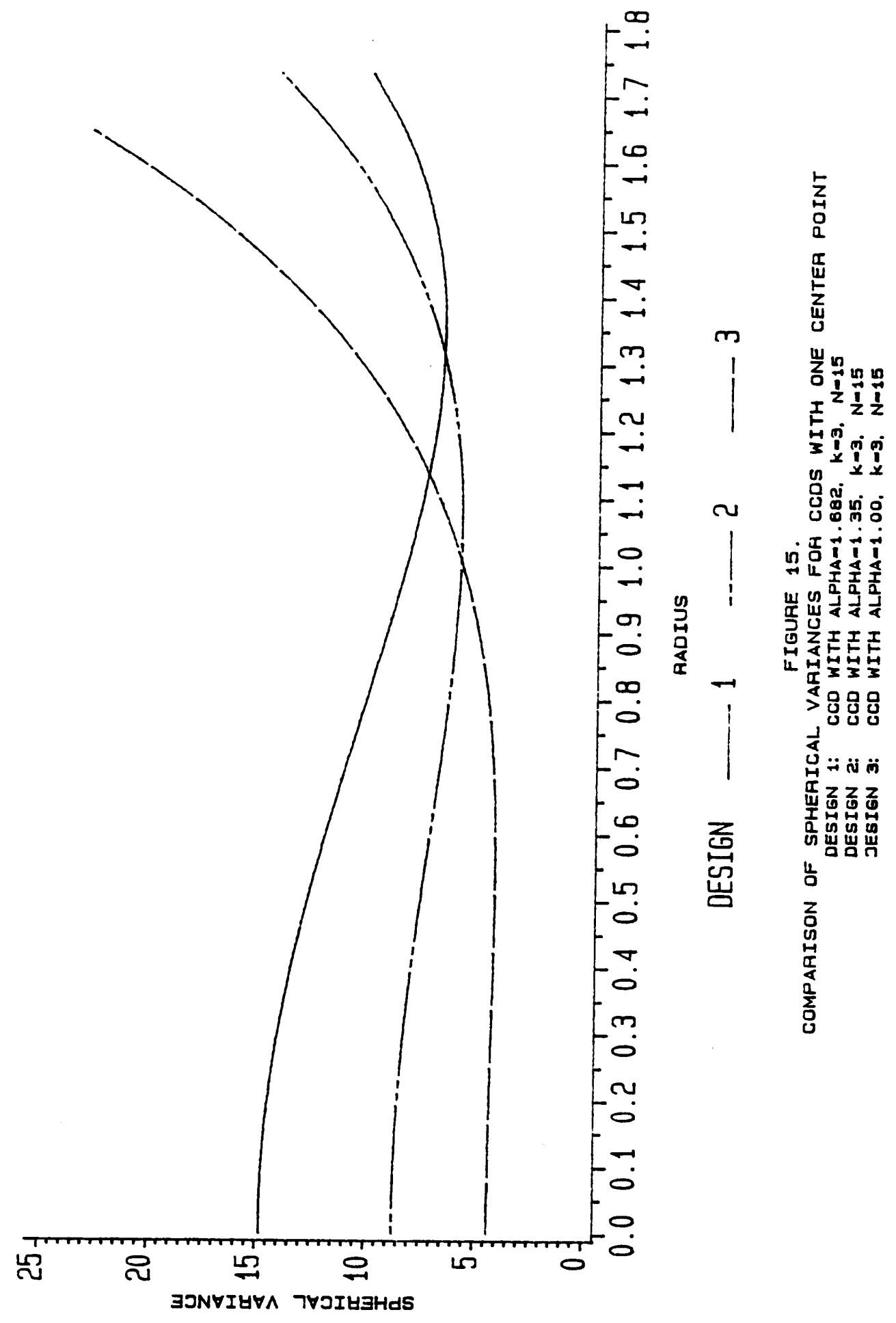




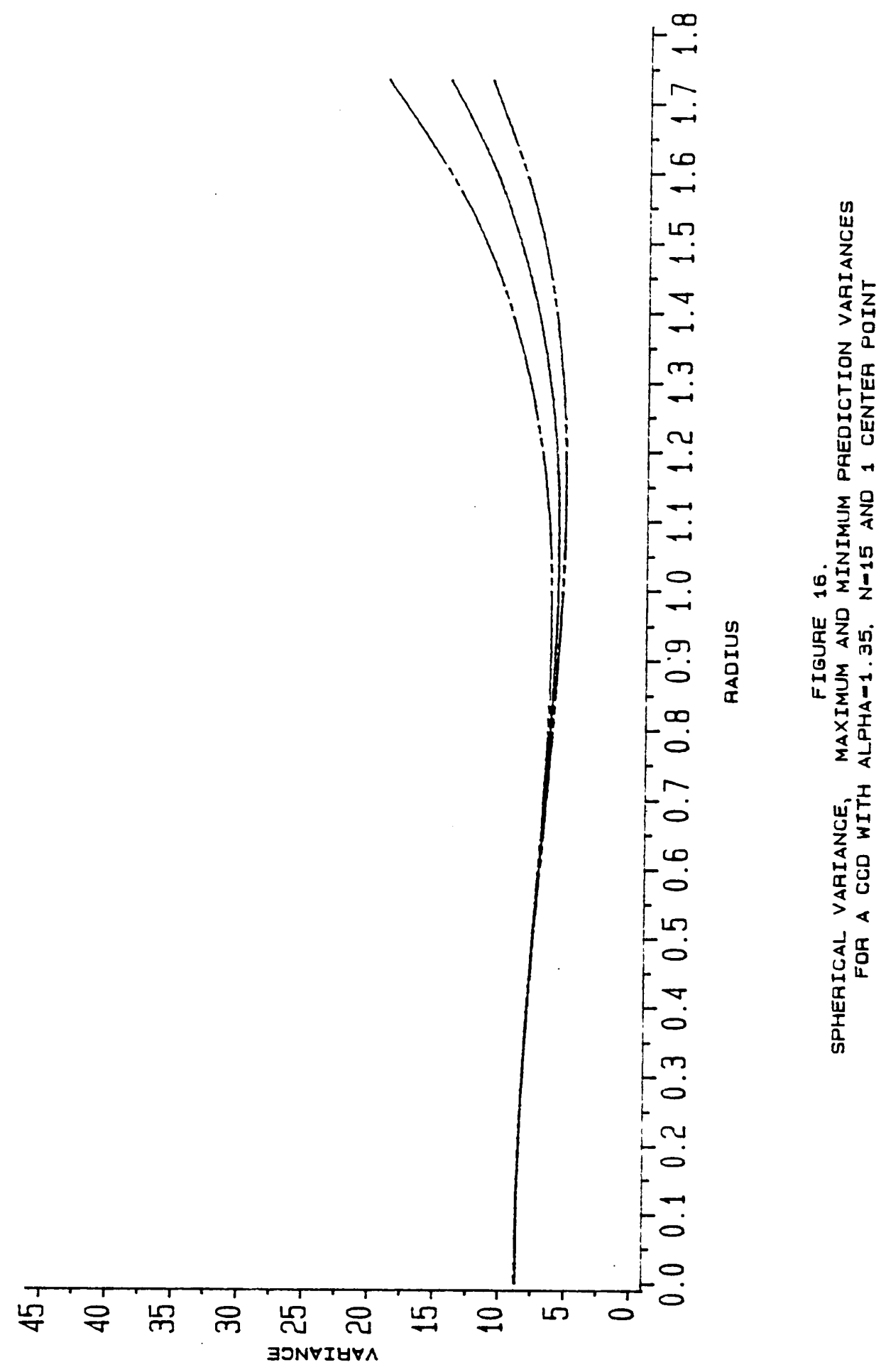




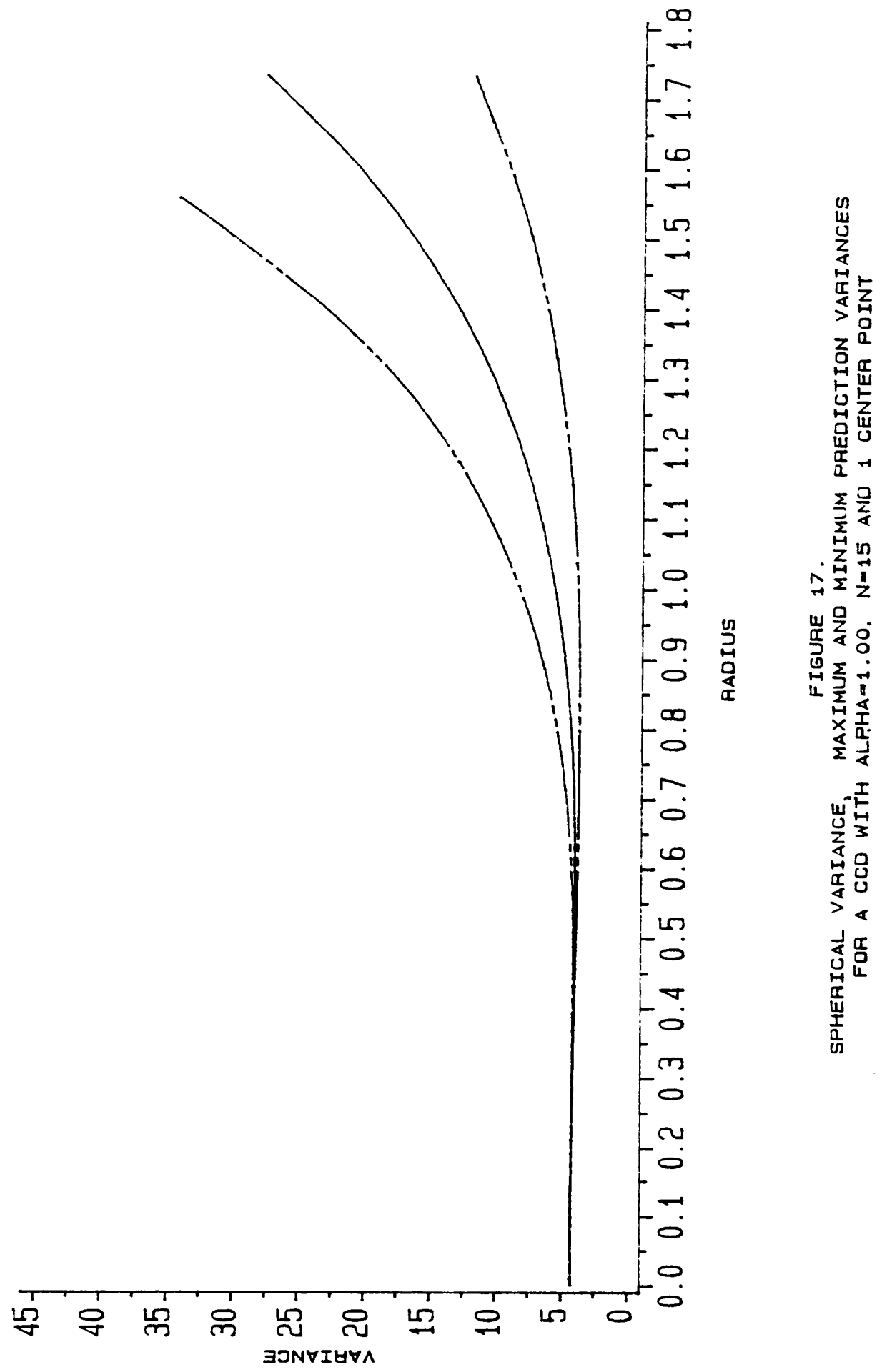


than -1 and no more than +1 , that is, at locations on or within the unit cube. If this is the case, the best and most logical choice of design from among the three ccds is the ccd with $\alpha=1.0$.

When there is sizeable dispersion in the prediction variances on spheres, it is of interest to know at what locations the response is being poorly estimated and where the response is being well estimated. For both the non-rotatable example designs the worst prediction variance on a sphere occurs where two of the variables are at level zero and the third is at a level equal to + or - the radius of the sphere for all radii. That is, the maximum prediction variances occur on vectors from the origin through the axial points of the design. On the other hand, the minimum prediction variance on a sphere is found at all locations for which the magnitudes of the three design variables are the same. For example, the most precise predicted values on the outermost sphere, $r=1.732$, occur at the locations $\left(\begin{array}{lll}x_{1} & x_{2} & x_{3}\end{array}\right)=\left(\begin{array}{lll}1 & 1 & 1\end{array}\right),\left(\begin{array}{lll}1 & 1 & -1\end{array}\right),\left(\begin{array}{lll}1 & -1 & 1\end{array}\right)$, etc. It has been found that the maximum and minimum prediction variances on spheres appear to be located at the same points regardless of the number of center runs affixed to the ccd.

The relatively poor estimation at locations around the center of the region for designs 1 and 2 can be improved by the addition of center runs to the design. For example, the spherical variance curves of Figure 18 indicate the effects of taking 1, 3, 4 or 6 experimental runs in the center of the rotatable ccd. Since this design is rotatable, the spherical variance for a sphere actually represents the prediction variances at every location on that sphere. The rotatability property of equal variances on spheres is not lost by the addition of center runs to the design.

Taking 3 or 4 center runs results in comparable prediction variances to those obtained with design $3,\left(\alpha=1.0, n_{0}=1\right)$, on spheres about the region center to a radius of about 1 . However, the quality of prediction obtained with the rotatable ccd is still much better when predicting further from the center. Six center runs do not appear to greatly improve the prediction variances about the region center over the improvement resulting from the addition of 3 or 4 center points. 


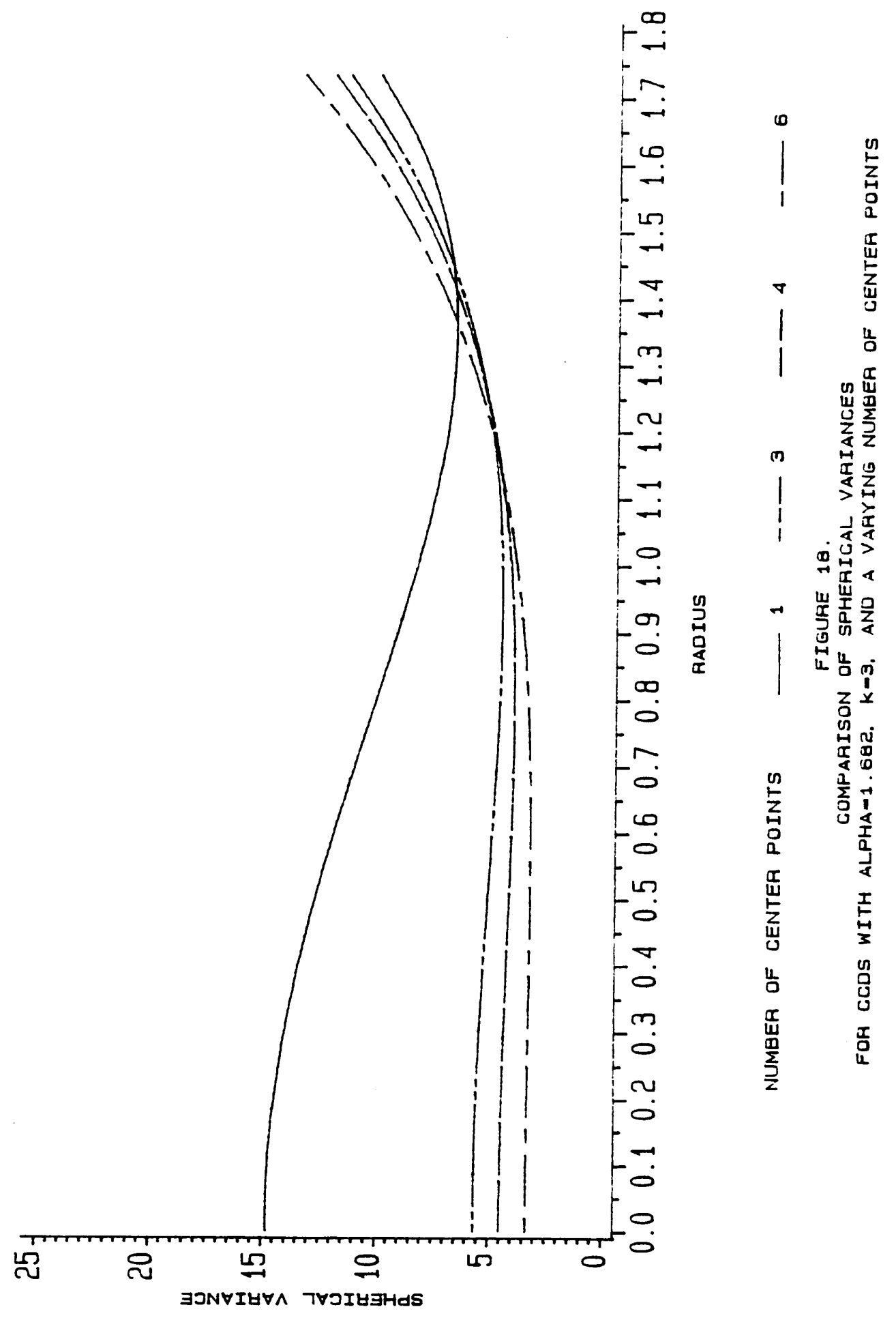


In general, for any $\alpha$-value, additional experimental runs taken at the region center yield increased precision in the predicted values at locations near the center. As an increasing number of center points are taken, the variances at locations on the outskirts of the region appear to worsen due to the additional cost involved in taking the extra runs. Figure 19 illustrates the improvement in the prediction capability of the three example ccds when 3 center points are taken rather than just one. The improvement in the prediction variances at locations near the region center is not as dramatic for design 3 , with $\alpha=1.0$, as for the designs with larger axial values. Considering the dispersion in the variances on spheres for the three designs, which has not been changed by the additional center runs, designs 1 and 2 are clearly preferred over design 3 when 3 runs are taken at the region center.

\subsubsection{Comparison of a Box-Behnken Design with Two CCDs}

The researcher may choose to use a Box-Behnken design (section 2.4.3) rather than a somewhat larger ccd in some experimental situations. In particular, this may be the case if it is desired that no more than three levels of each design variable be used for experimentation. Box-Behnken designs are believed to be rotatable or nearly rotatable. Lucas (1976) has shown that they have high D-efficiencies in a sphere of radius 1.

The prediction capability of a three variable Box-Behnken design is depicted in Figure 20. This particular design has one center run. More runs taken at the center would improve the prediction variance picture on spheres close to the region center. Note that comparatively little dispersion in the variances on spheres about the design center, which is the region center, is present, especially compared to the $\operatorname{ccd}$ with $\alpha=1.0$. 


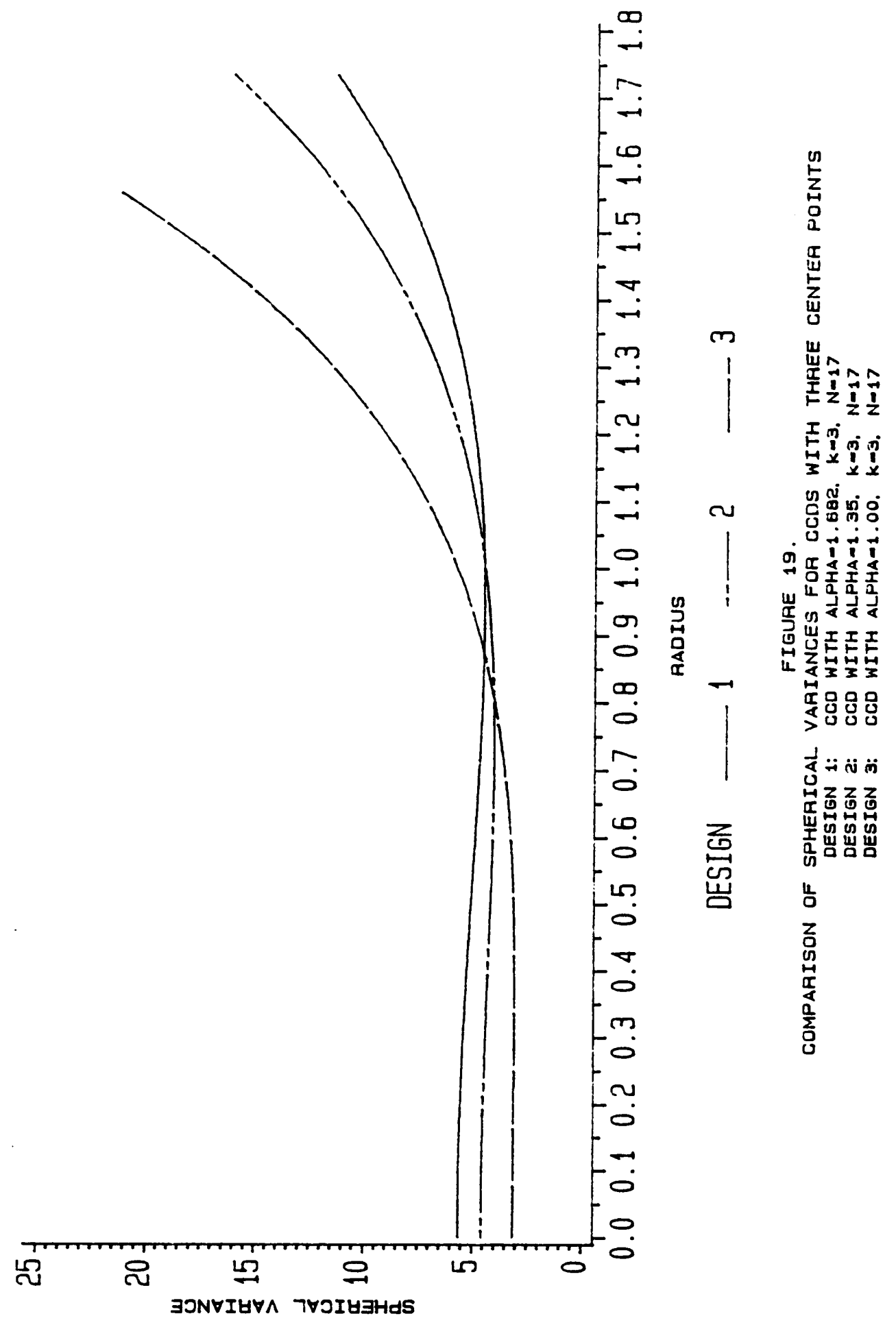




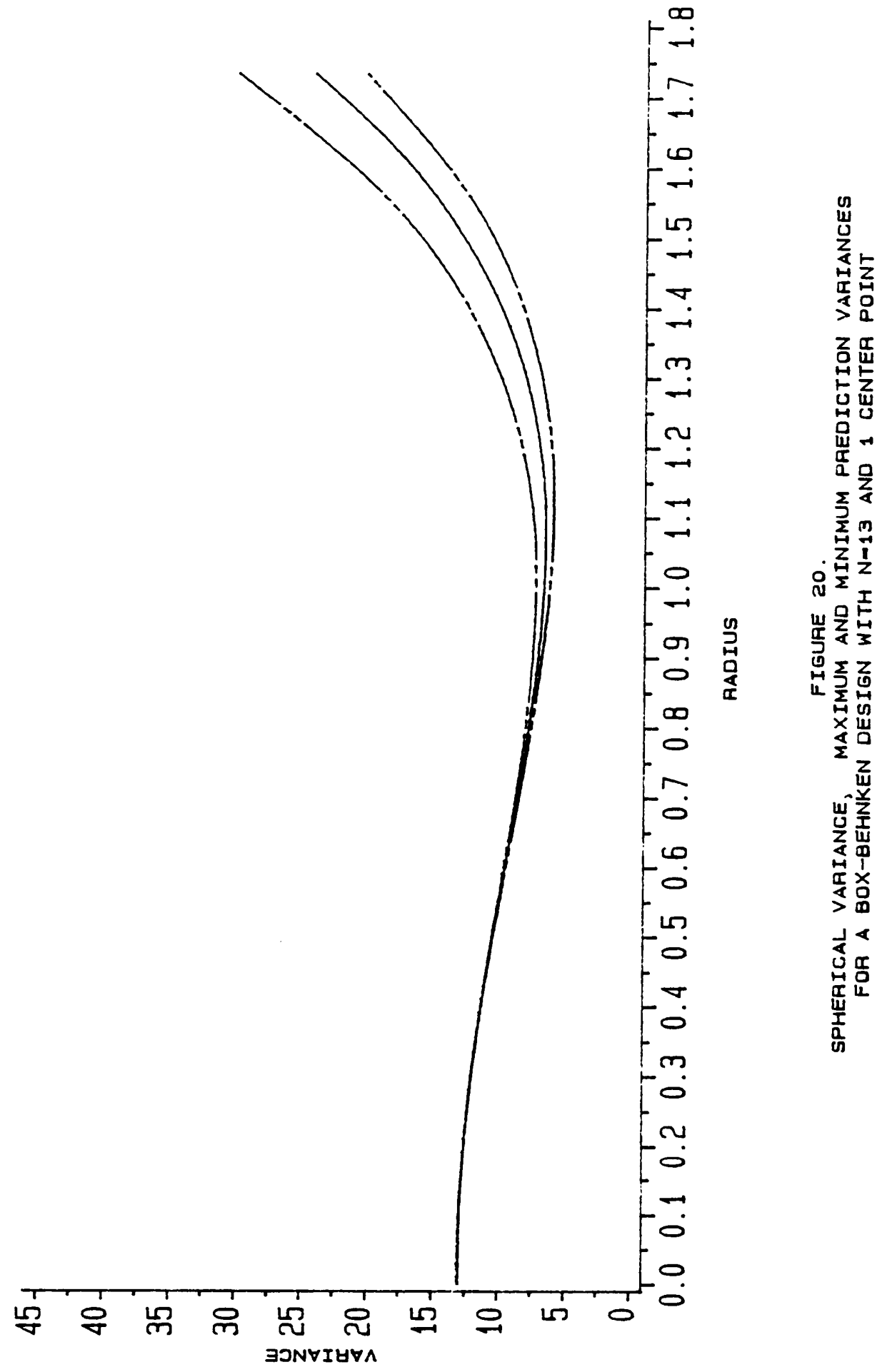


The prediction capability of the Box-Behnken design with one center point is compared to that of a rotatable ccd $(\alpha=1.682)$ and a three level $\operatorname{ccd}(\alpha=1.0)$ in Figure 21. Both ccds have one center point and $N=15$ design points. The Box-Behnken design has 13 design points.

In the region near the center, from $r=0$ to 1 , the Box-Behnken design is somewhat better than the rotatable ccd. However, the ccd with $\alpha=1.0$ yields considerably better precision of the estimated responses in this region.

The average prediction variance on a sphere obtained with the ccd with $\alpha=1.0$ suffers considerably when estimating the response at locations which lie beyond a radius of 1 . On the outskirts of the region the Box-Behnken performs somewhat better on average than the ccd with $\alpha=1.0$. The rotatable ccd, which has information in this region provided by the axial and factorial points, fares much better in terms of prediction capability near the perimeter of the region.

As a competitor to the central composite designs the Box-Behnken design has the advantage of requiring a fewer number of experimental runs than a ccd and only three levels of the design variables. As with the ccds, the prediction variances at locations near the region center could be improved with additional experimental runs taken at the center of the region. The spherical variance comparisons of Figure 21 a show the improvement afforded by the addition of three more center runs to the Box-Behnken design with one run in the center. The relatively slight dispersion in the prediction variances on spheres is a positive feature of the Box-Behnken design. This consistent accuracy of prediction on spheres is not effected by the addition of center points to the design.

\subsubsection{Robustness of a Rotatable CCD to Loss of Design Points}

The purpose of this section is to use the spherical variance and maximum and minimum prediction variances to examine the effects of losing design points during experimentation on the 


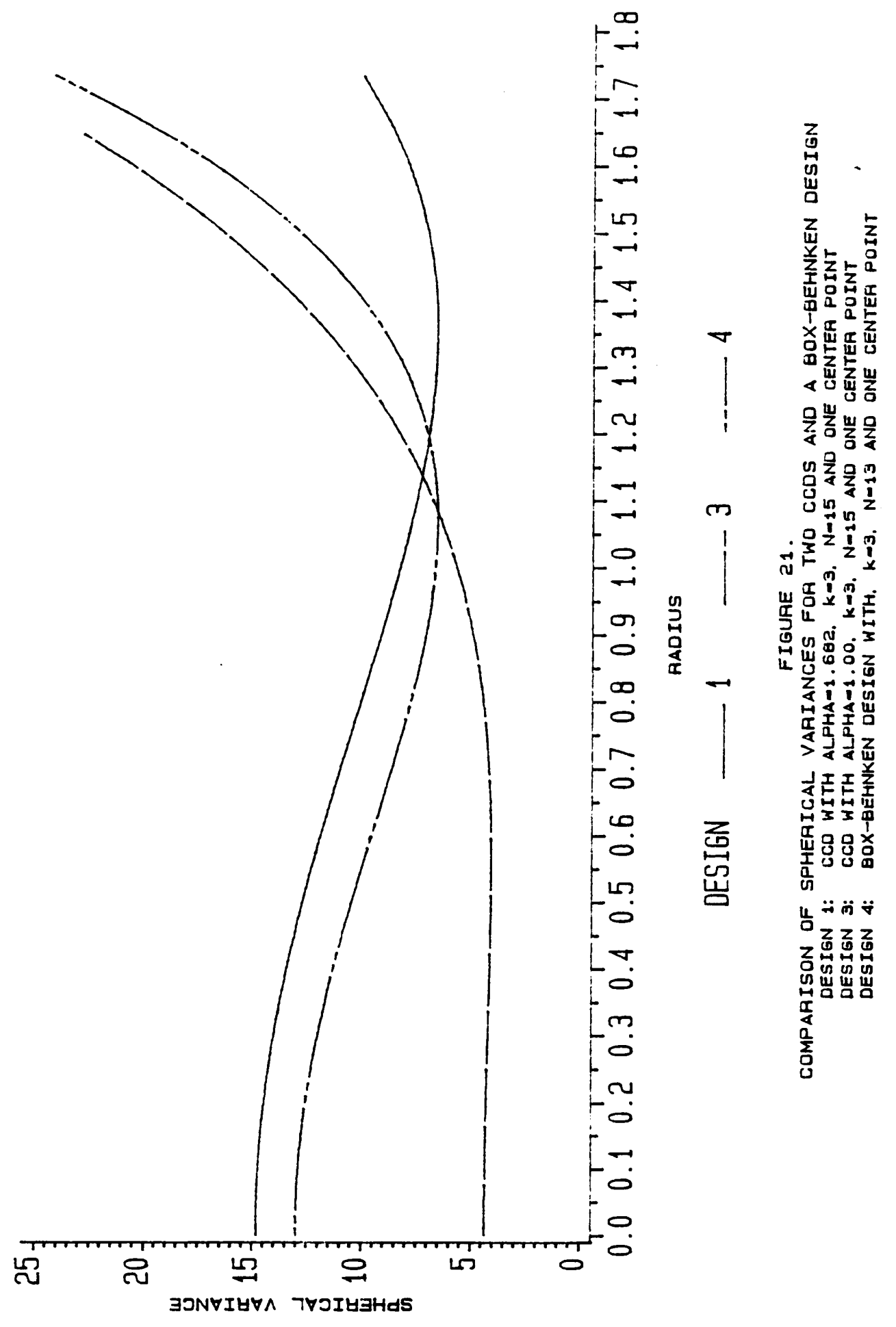




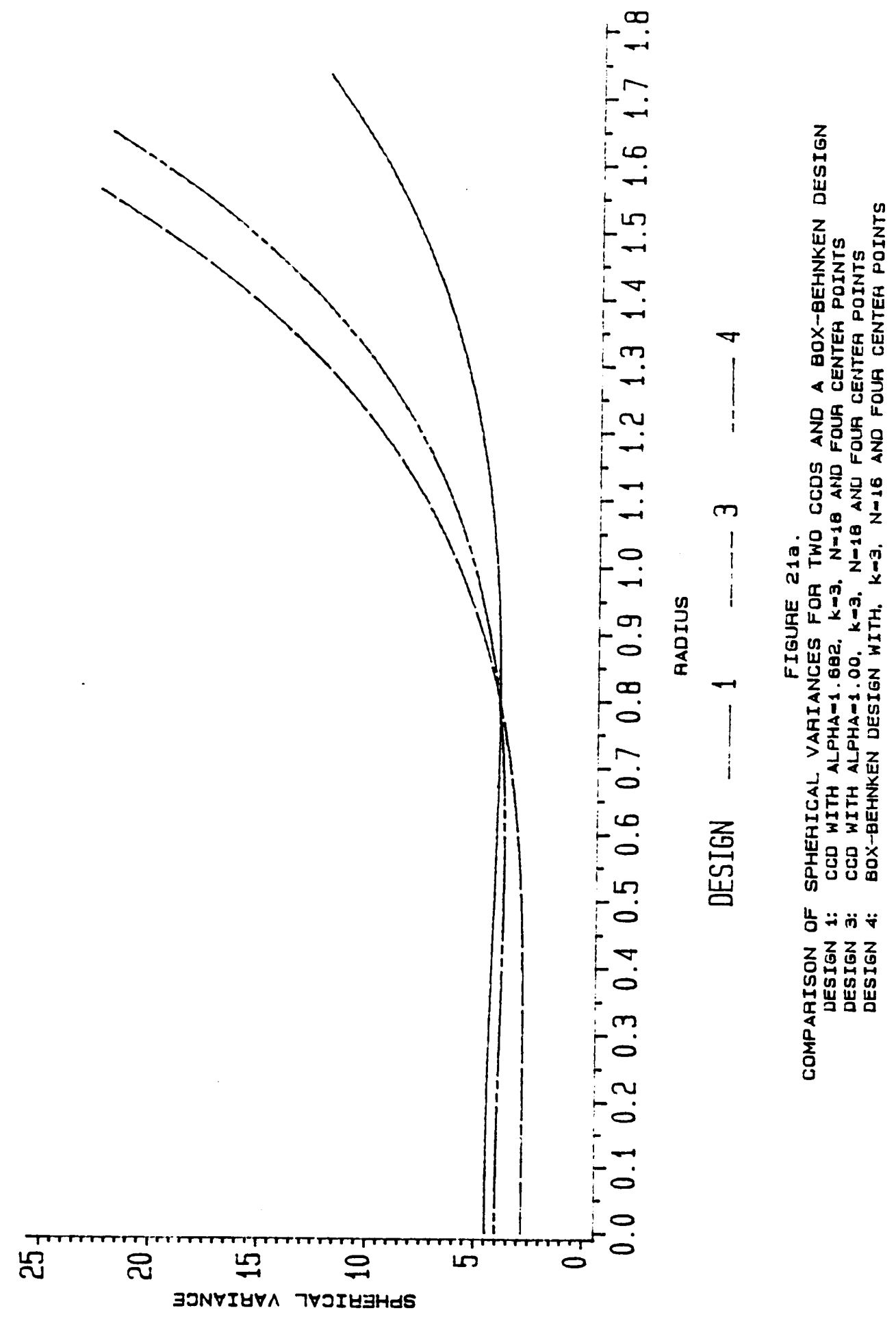


prediction capability of a central composite design. A ccd with design parameters $\alpha=1.682$ and $n_{0}=3$ has been chosen for the illustration. This design is rotatable, however, rotatability will be lost when points are dropped from the design.

Initially, consider the loss of one of the axial points, specifically, the point $\left(\begin{array}{lll}-1.682 & 0 & 0\end{array}\right)$, from the design. The resulting change in the quality of prediction on spheres is depicted in Figure 22. The prediction variances have not been weighted by the size of the designs in this case. The original prediction variance curve for the complete ccd is indicated by the ${ }^{*}$ symbol on the graph. It is very nearly identical to the minimum prediction variance curve for the design after the axial point has been lost. At some locations at least the quality of prediction is maintained.

On the average the prediction variances have not been greatly disturbed by the loss of the point. The greatest loss of precision occurs for the estimated responses at the locations of maximum prediction variance. The locations of maximum prediction variance have been computed through the MINOS algorithm (section 3.2.4) for radii to $\sqrt{3}$. These locations turn out to be on a line from the origin through the location of the lost axial point - exactly where one might expect the greatest loss in precision to occur. Judging from the small average prediction variances on spheres, the prediction variances at most other locations appear to be little changed by the loss of the point.

Now, suppose a second axial point is lost during the experiment. In this example, the points $\left(\begin{array}{lll}-1.682 & 0 & 0\end{array}\right)$ and $\left(\begin{array}{llll}0 & 0 & 1.682\end{array}\right)$ have been dropped from the design. Figure 23 shows the spherical variance, maximum and minimum variance curves for the depleted design. The prediction variance curve obtained with the full ccd is included for comparison. The prediction capability of the design has not diminished substantially from that of the design with one axial point lost, (see Figure 22; the * symbol again denotes the original prediction variance curve of the complete ccd).

With the loss of two axial points the prediction capability of the ccd has weakened at locations on the lines through the region center which go through the lost axial points. The largest 


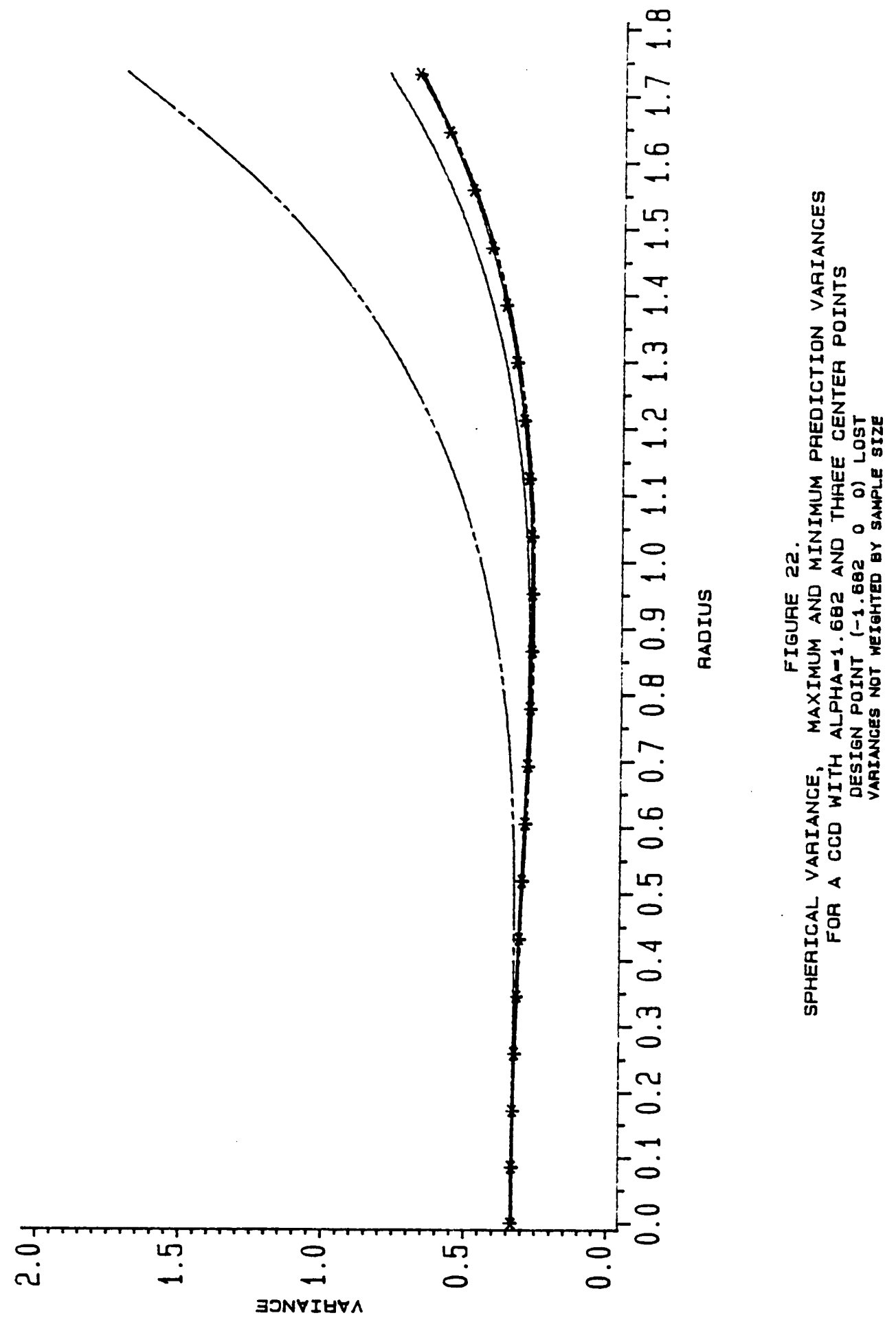




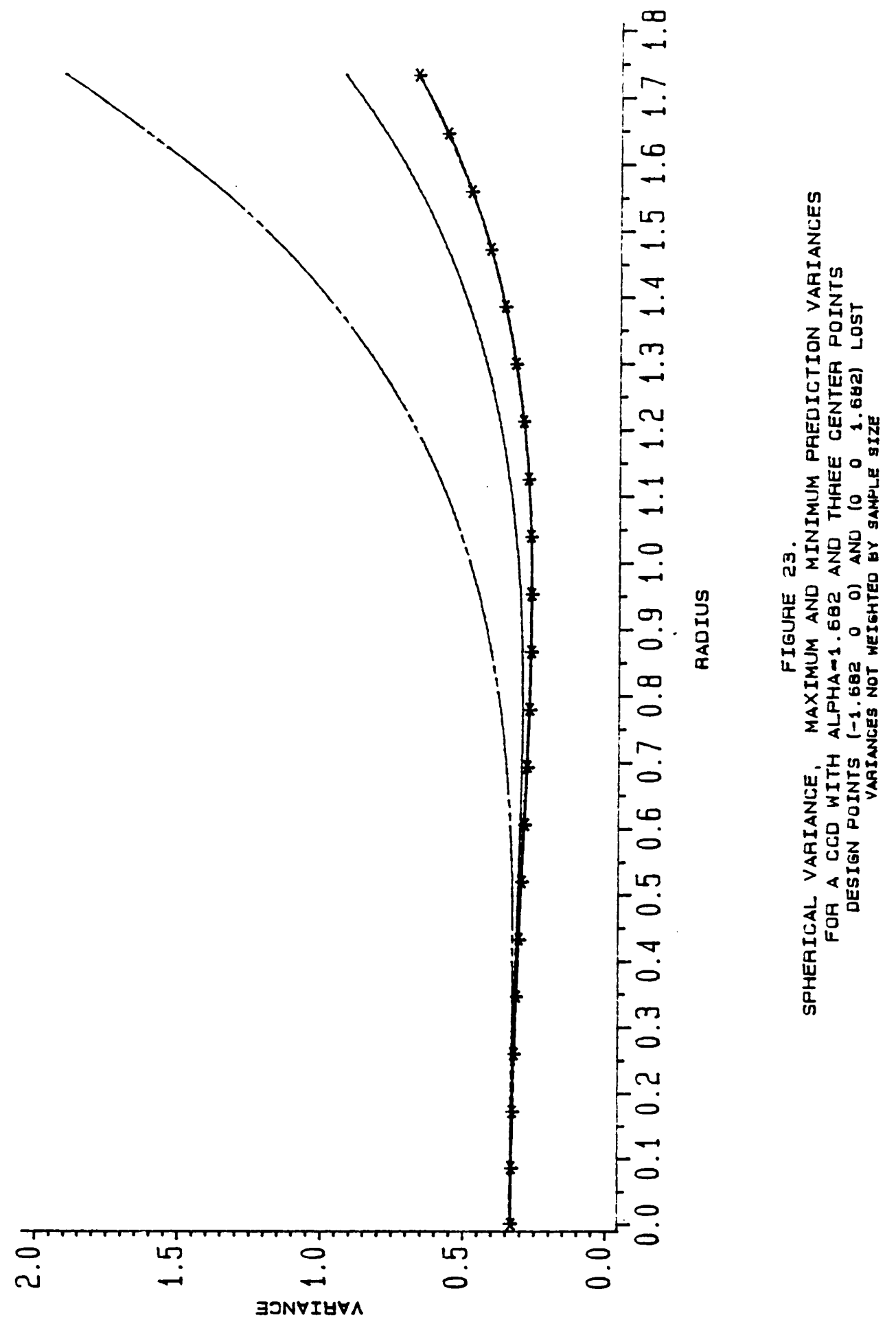




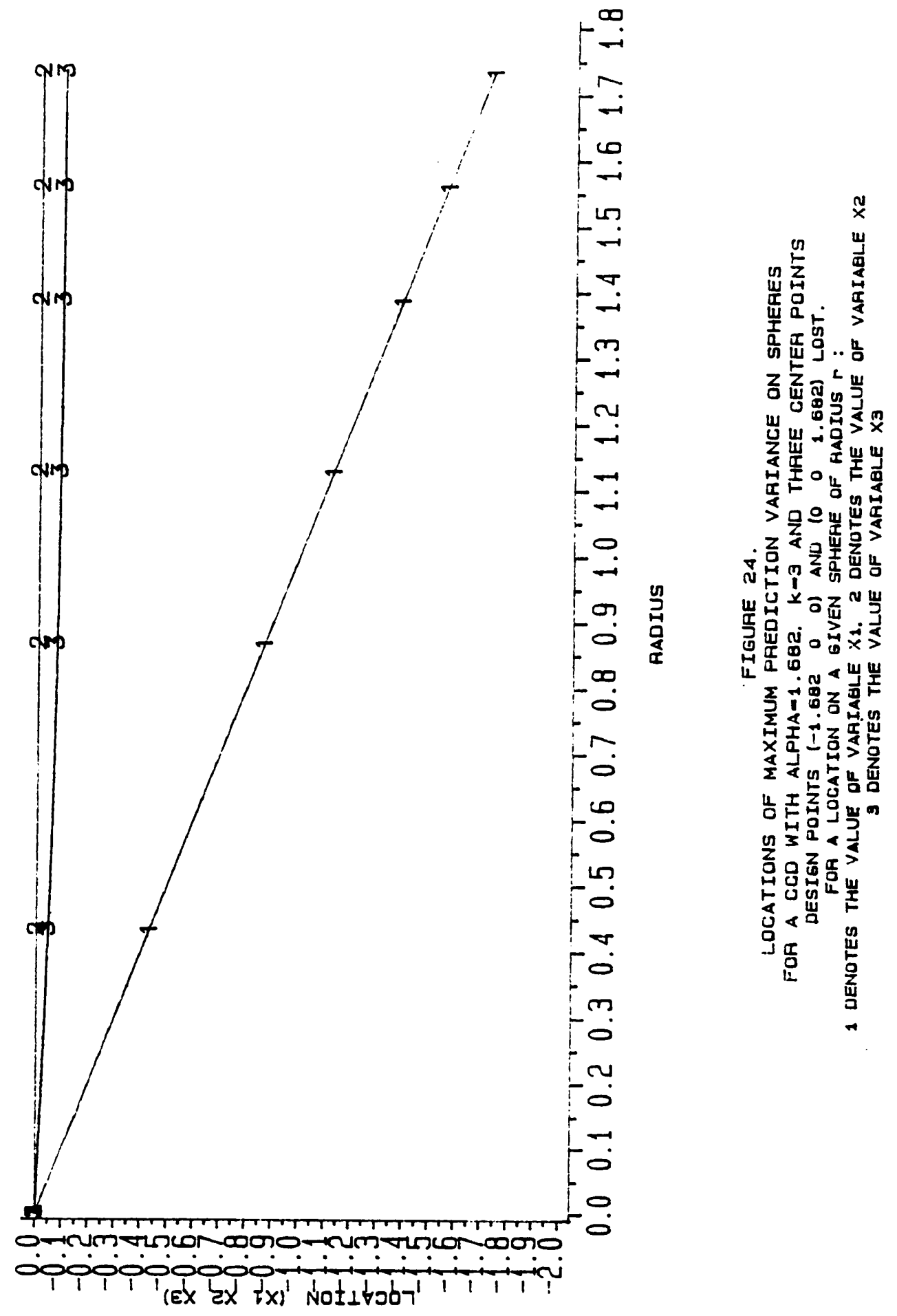


prediction variances, however, occur at the locations given in Figure 24. The maximum prediction variances on spheres also occur at another set of locations. In this set, the roles of $x_{1}$ and $x_{3}$ are interchanged and all levels are now positive. These locations are on lines which are close to the lines going through the lost axial points.

The results of Figures 22 and 23 indicate that the prediction variances at locations near the center of the region are not appreciably changed by the loss of the axial points. Although some prediction capability is lost at locations in lines through the origin in the general directions of the lost axials, the prediction variances there may still be considered reasonable. In conclusion, the quality of the predicted responses obtained with the rotatable ccd is found to be fairly robust to the loss of one or two axial points.

The illustrations of this section by no means form an extensive study of the prediction capability and robustness to loss of design points of the family of central composite designs. The purpose of this section was to show how the graphical method may be used to assess the prediction capability and robustness to loss of design points of a ccd. The illustrations presented throughout the chapter, while selected to give some applications of the graphical method which may prove to be useful to the experimenter, are intended to show the use and interpretation of the prediction variance functions and their graphical representation.

\subsection{Minimum and Nearly Minimum Point Designs}

\subsubsection{A Comparison of Small Composite and Hybrid Designs}

The small composite and hybrid designs were both developed as economical alternatives to central composite designs. Thus, it is natural to compare the prediction capabilities of the two 
design types. A three variable small composite design (scd) such as that listed in section 2.4 .4 with $\alpha=\sqrt{3}$, and the hybrid designs identified as 310 and $311 \mathrm{~A}$ by Roquemore (1976) (see section 2.4.5) have been selected for comparison. Each design has one center run and $N=11$ design points. The number of design points is just one over the minimum number required to estimate the parameters of a second order model in three variables. The three variable hybrid designs 310 , $311 \mathrm{~A}$ and $311 \mathrm{~B}$ appear in Table 5.

Spherical variance comparisons of the three designs appear in Figure 25. It is evident that overall the scd does not perform as well as the hybrid designs. The hybrid 310 plus one center point design predicts extremely well near the region center. However, its average prediction capability quickly diminishes as one predicts on spheres further from the region center. The $311 \mathrm{~A}$ design, on the other hand, predicts poorly at locations near the center, but relatively well at locations near the periphery of the region.

Comprehensive pictures of the prediction capability on spheres are presented in Figure 26 for the scd, 27 for the hybrid 310 and in Figure 28 for the hybrid $311 \mathrm{~A}$ design. From the plots it is seen that the scd provides the least consistent estimation of the response on spheres. There is also a sizeable amount of dispersion in the prediction variances on the outer spheres of the region obtained with the 310 design. The variances of the estimated responses are nearly equal on spheres throughout the region for the hybrid $311 \mathrm{~A}$ design.

For the hybrid 310 design the poorest prediction variances on the outer spheres occur where the $x_{3}$-axis intersects the spheres. These are points along the lines through the origin directed at the axial points $\left(\begin{array}{lll}0 & 0 & \alpha\end{array}\right)$ and $\left(\begin{array}{lll}0 & 0 & -\alpha\end{array}\right)$. The variances at these locations are slightly higher when $x_{3}$ is positive. The best prediction variances on the outer spheres can be found at locations for which $x_{1}$ and $x_{2}$, the variables involved in the two variable ced portion of the hybrid, are the same, and the level of the third variable is slightly smaller in magnitude than the other two. 
Table 5.

Roquemore's Hybrid Designs in Three Variables

\begin{tabular}{rrr|rrr|rrr} 
& $310^{*}$ & \multicolumn{3}{|c|}{$311 \mathrm{~A}$} & \multicolumn{3}{|c}{$311 \mathrm{~B}$} \\
\hline 0 & 0 & 1.2906 & 0 & 0 & 2 & 0 & 0 & $\sqrt{6}$ \\
0 & 0 & -.1360 & 0 & 0 & -2 & 0 & 0 & $-\sqrt{6}$ \\
-1 & -1 & 0.6386 & $-\sqrt{2}$ & $-\sqrt{2}$ & 1 & -0.7507 & 2.1063 & 1 \\
1 & -1 & 0.6386 & $\sqrt{2}$ & $-\sqrt{2}$ & 1 & 2.1063 & 0.7507 & 1 \\
-1 & 1 & 0.6386 & $-\sqrt{2}$ & $\sqrt{2}$ & 1 & 0.7507 & -2.1063 & 1 \\
1 & 1 & 0.6386 & $\sqrt{2}$ & $\sqrt{2}$ & 1 & -2.1063 & -0.7507 & 1 \\
1.1736 & 0 & -0.9273 & 2 & 0 & -1 & 0.7507 & 2.1063 & -1 \\
-1.1736 & 0 & -0.9273 & -2 & 0 & -1 & 2.1063 & -0.7507 & -1 \\
0 & 1.1736 & -0.9273 & 0 & 2 & -1 & -0.7507 & -2.1063 & -1 \\
0 & -1.1736 & -0.9273 & 0 & -2 & -1 & -2.1063 & 0.7507 & -1 \\
& & & 0 & 0 & 0 & 0 & 0 & 0
\end{tabular}

* The hybrid 310 design does not require a center run to have a nonsingular $X^{\prime} X$ matrix. For the illustrations of section 5.2.1 a center run is affixed to the hybrid 310 design. 


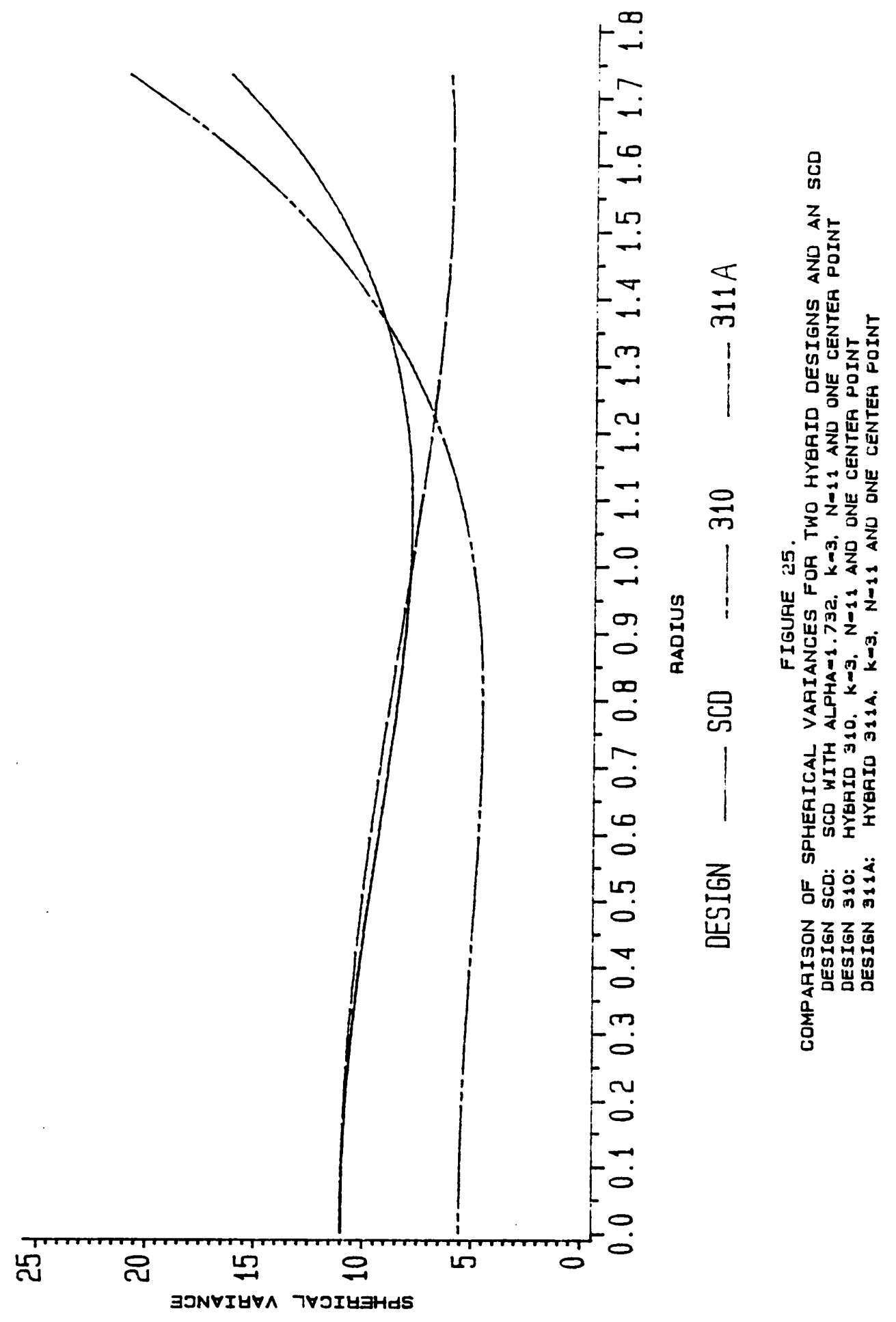




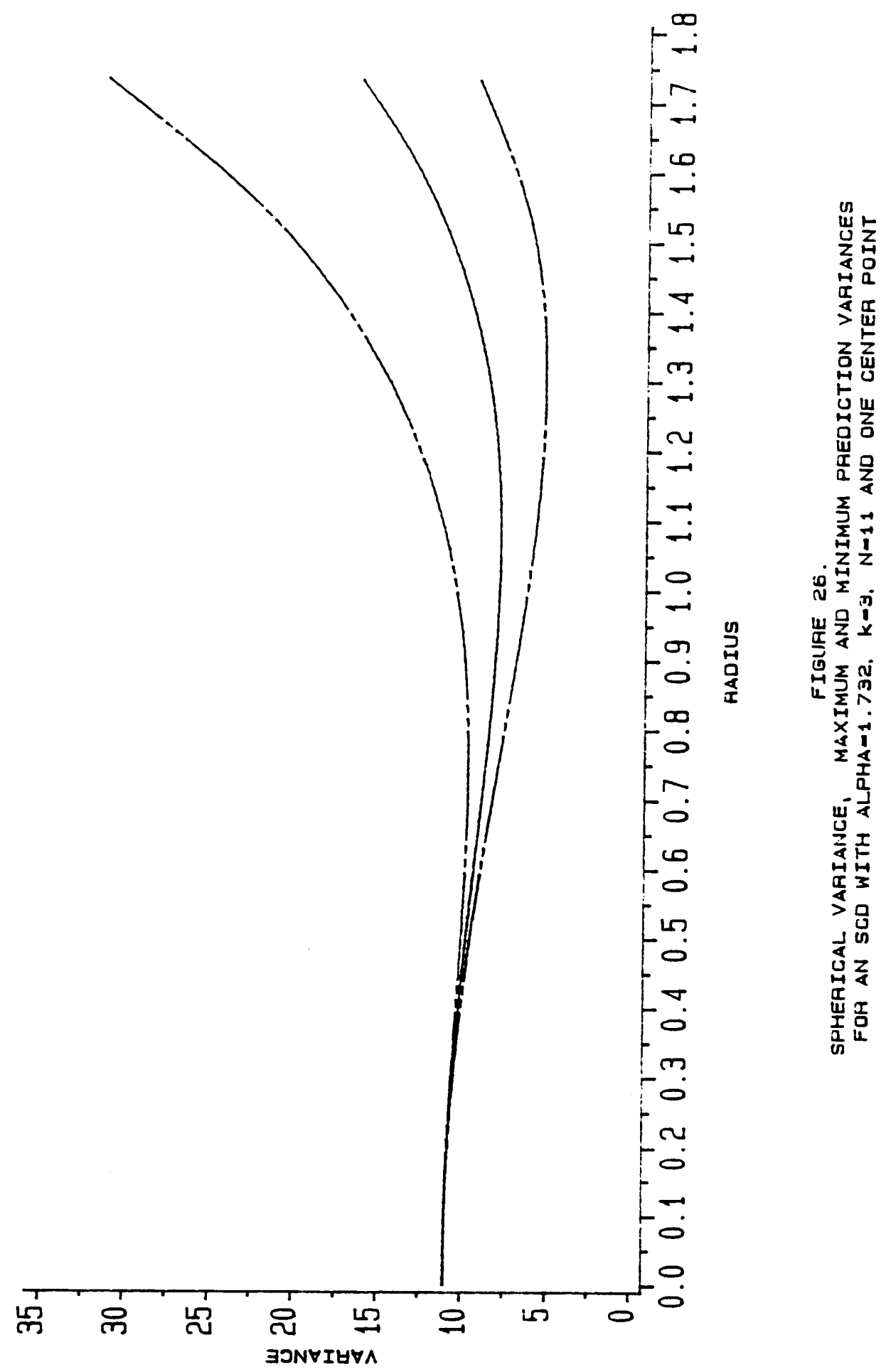




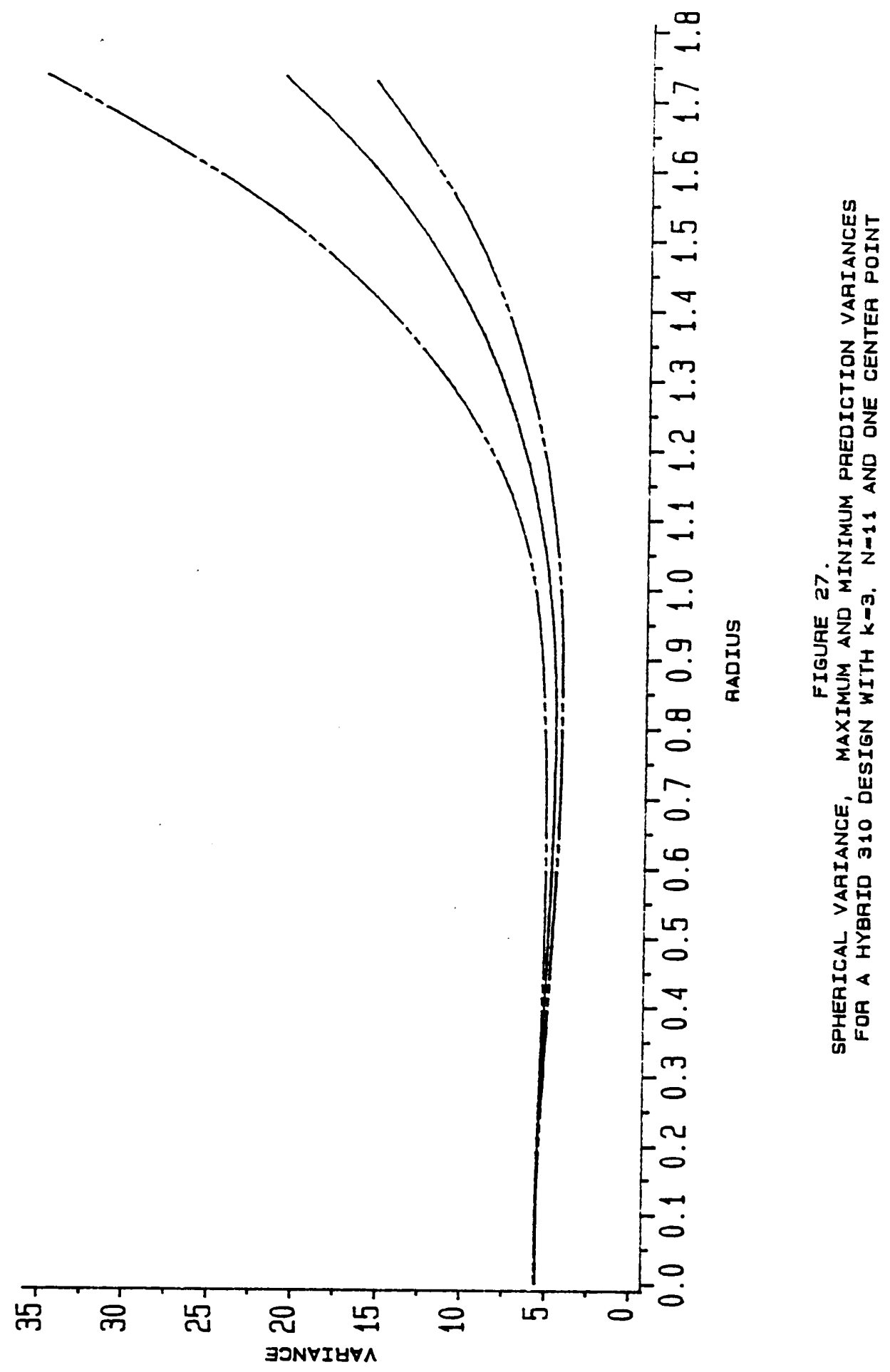




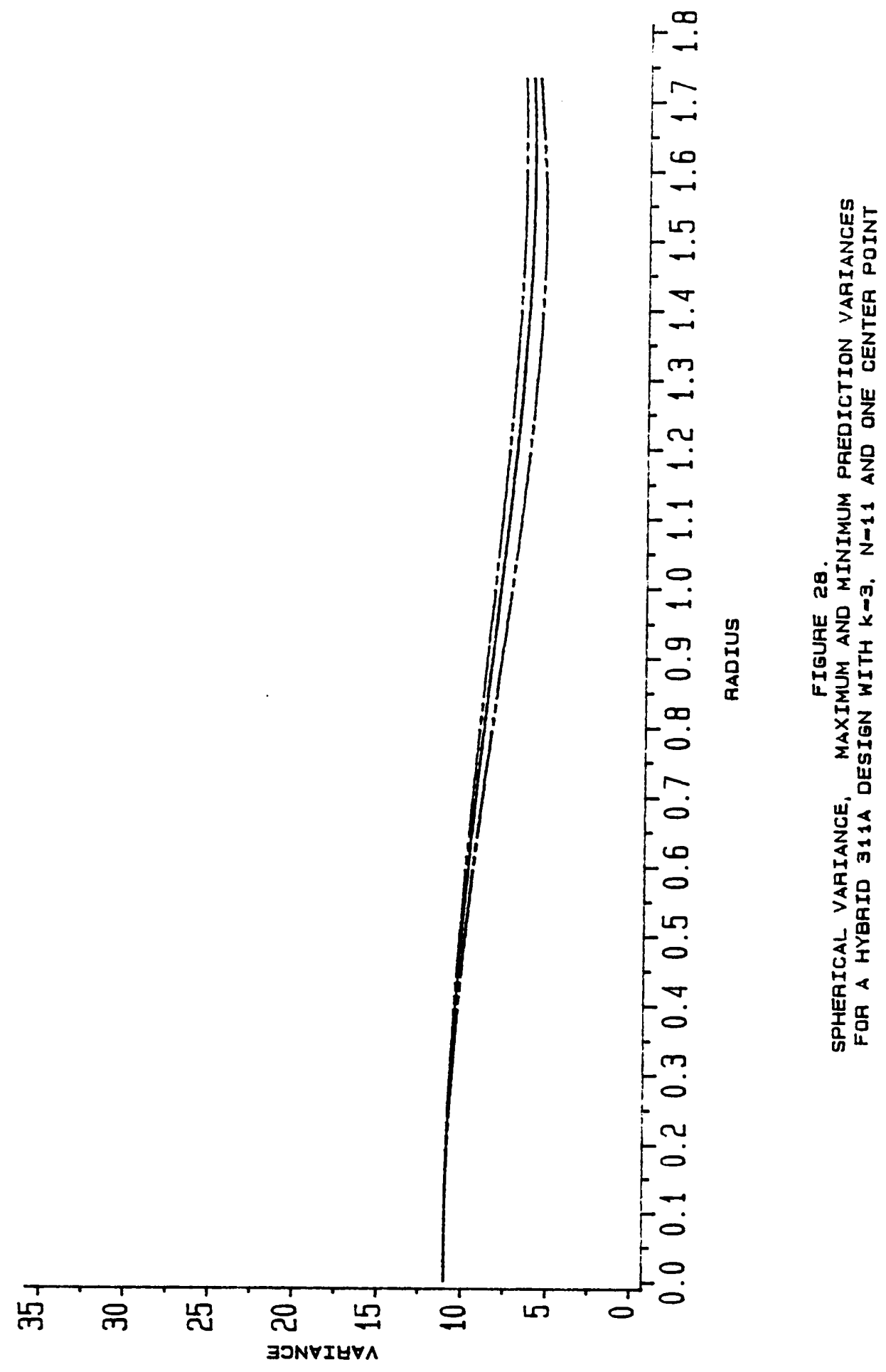


It is interesting that for the scd the largest prediction variance on a sphere of radius $r$ corresponds to the response at the locations $(a a-a), \quad(a-a a)$, $\left(\begin{array}{cc}-a & a\end{array}\right)$ and ( $\left.-a-a-a\right)$, for $a>0$, on the sphere. That is, the points of maximum prediction variance on a sphere lie on lines from the origin through the factorial points which were not included in the fractional factorial portion of the scd. Analogously, the locations of the best estimated responses on a sphere are found where vectors from the origin through the factorial points of the scd intersect the sphere.

\subsubsection{A Comparison of Some Small D-Efficient Designs}

The prediction capability of the economical designs due to Box and Draper (1971, 1974), Hoke (1974) and Notz (1982) will now be considered. The three variable Box-Draper design is given in section 2.4.8. The Notz design is listed in section 2.4.6. The Hoke design selected for comparison is the design commonly referred to as Hoke D2. This design is the one listed in section 2.4.7.

These particular designs were chosen for comparison because they have comparable Defficiencies on a unit hypercube. The D-efficiencies of the three designs are presented in the chart below. It is of interest to know if they also have similar predicting abilities throughout the region under consideration.

\section{D-efficiencies for example designs}

$\begin{array}{lcc}\text { Design } & \text { N } & \text { D-efficiency ( \%) } \\ & \\ \text { Box-Draper } & 10 & 89.2 \\ \text { Hoke D2 } & 10 & 84.3 \\ \text { Notz } & 11 & 84.3\end{array}$


The spherical variance curves are presented for comparison in Figure 29. The maximum and minimum prediction variance curves along with the $V^{r}$ appear in Figures 30, 31 and 32.

On spheres which lie within the unit cube the designs have similar prediction variance pictures, with the Box-Draper design performing somewhat better than the other two. A comparison with the spherical variance curves of Figure 15 indicates that in this region these designs also perform well compared to some of the ccds considered in section 5.1.1. The accuracy of prediction is fairly consistent on spheres inside the unit cube for the Box-Draper and Notz designs. However, there is notable dispersion in the prediction variances on spheres inside the unit cube and throughout the region obtained with the Hoke D2 design.

On the average, at least, the precision of the estimated responses on spheres outside the unit cube quickly diminishes for the example designs. This is not surprising since these designs were not constructed to predict the response in this region. They have no design points, and, consequently, no first-hand information, beyond the corners of the unit hypercube. The Hoke and Notz designs, in particular, do not perform well in this region. The prediction variances on spheres are very unstable under both designs. Clearly, the Box-Draper design has the best overall prediction capability throughout the region.

\subsubsection{Comparisons of Computer Generated D-Optimal Designs}

The remaining minimum point designs to be investigated in this section are the computer generated designs of Mitchell and Bayne (1978) and Welch (1982).

The designs listed in Table 1 of section 2.4 were generated to be D-optimal among all designs of the same size consisting of points on the unit cube. The D-efficiencies of the ten point designs are equal to $86.3 \%$. The pictures of the prediction capability of the MB10 and WC10 designs are nearly identical. Only the MB10 design will be used for illustration. The WD10 design, which has 


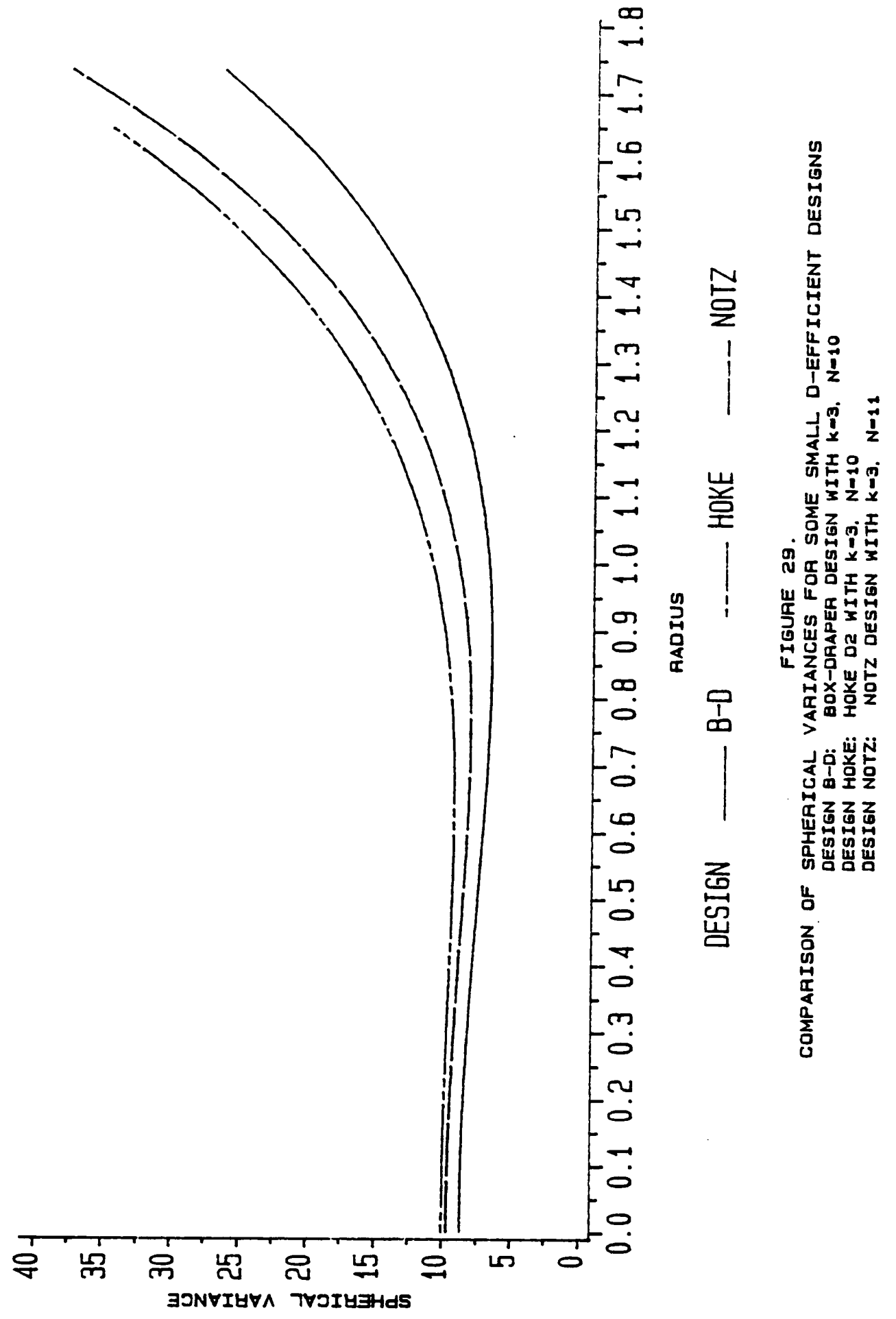




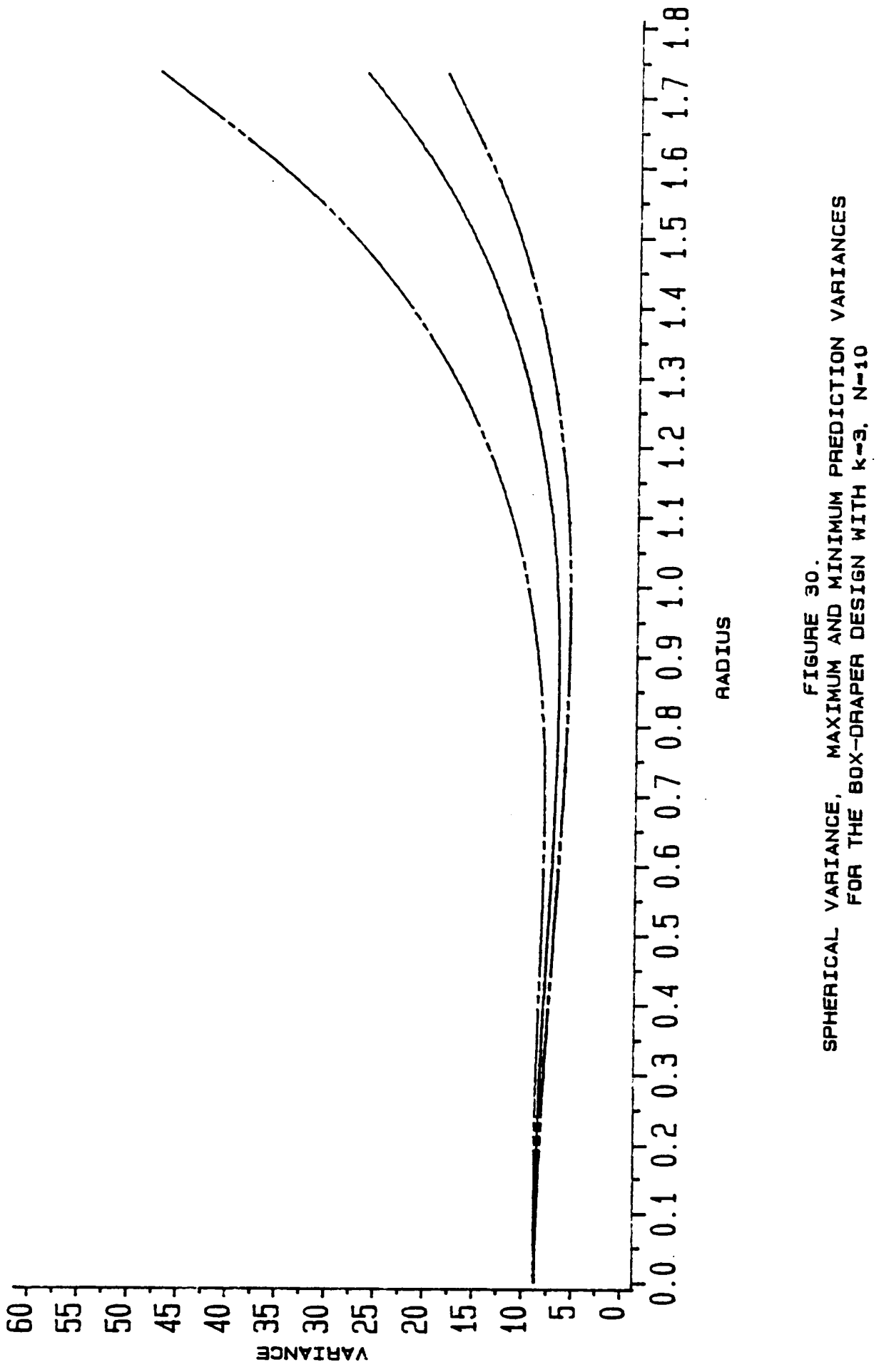




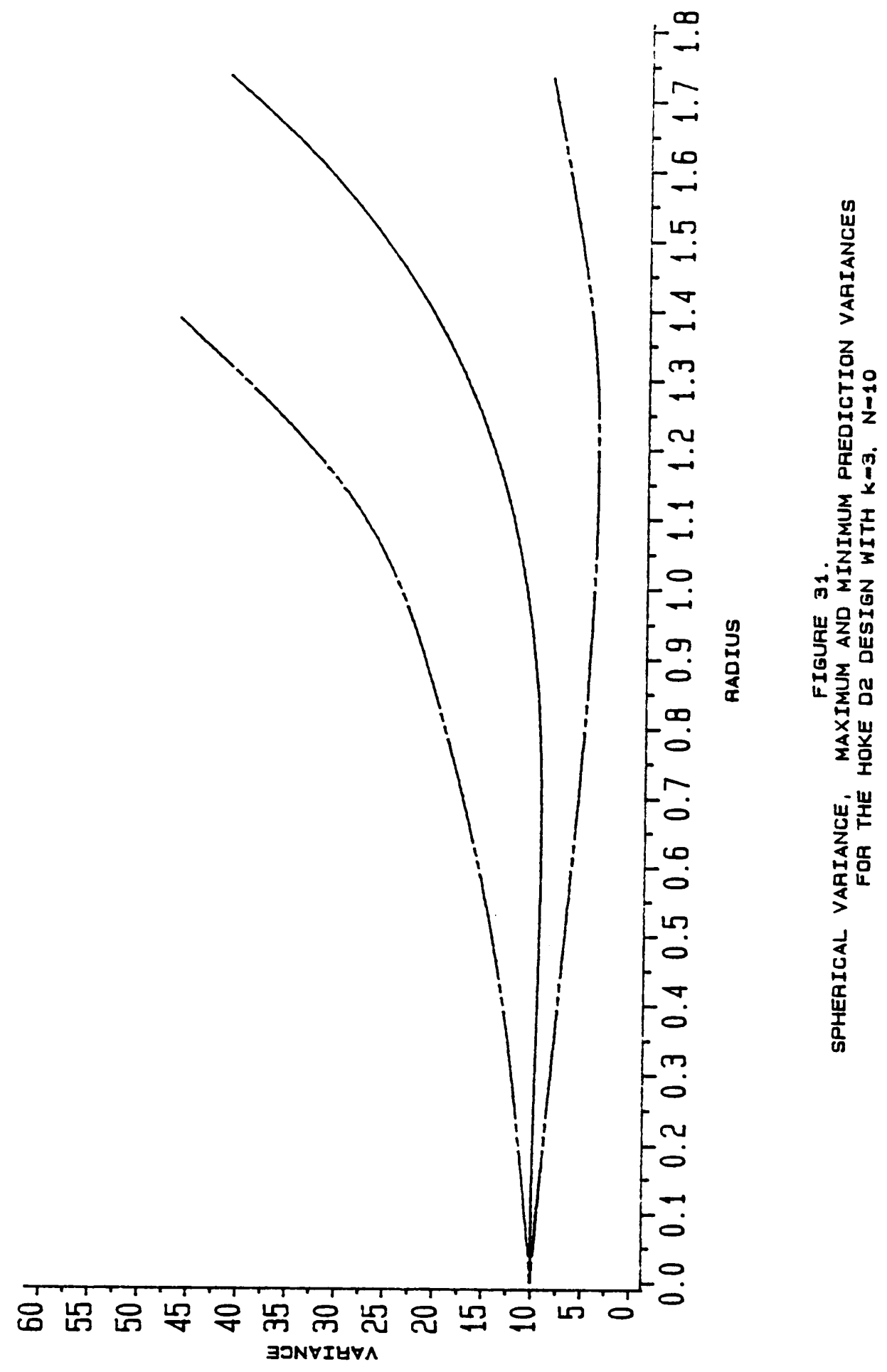




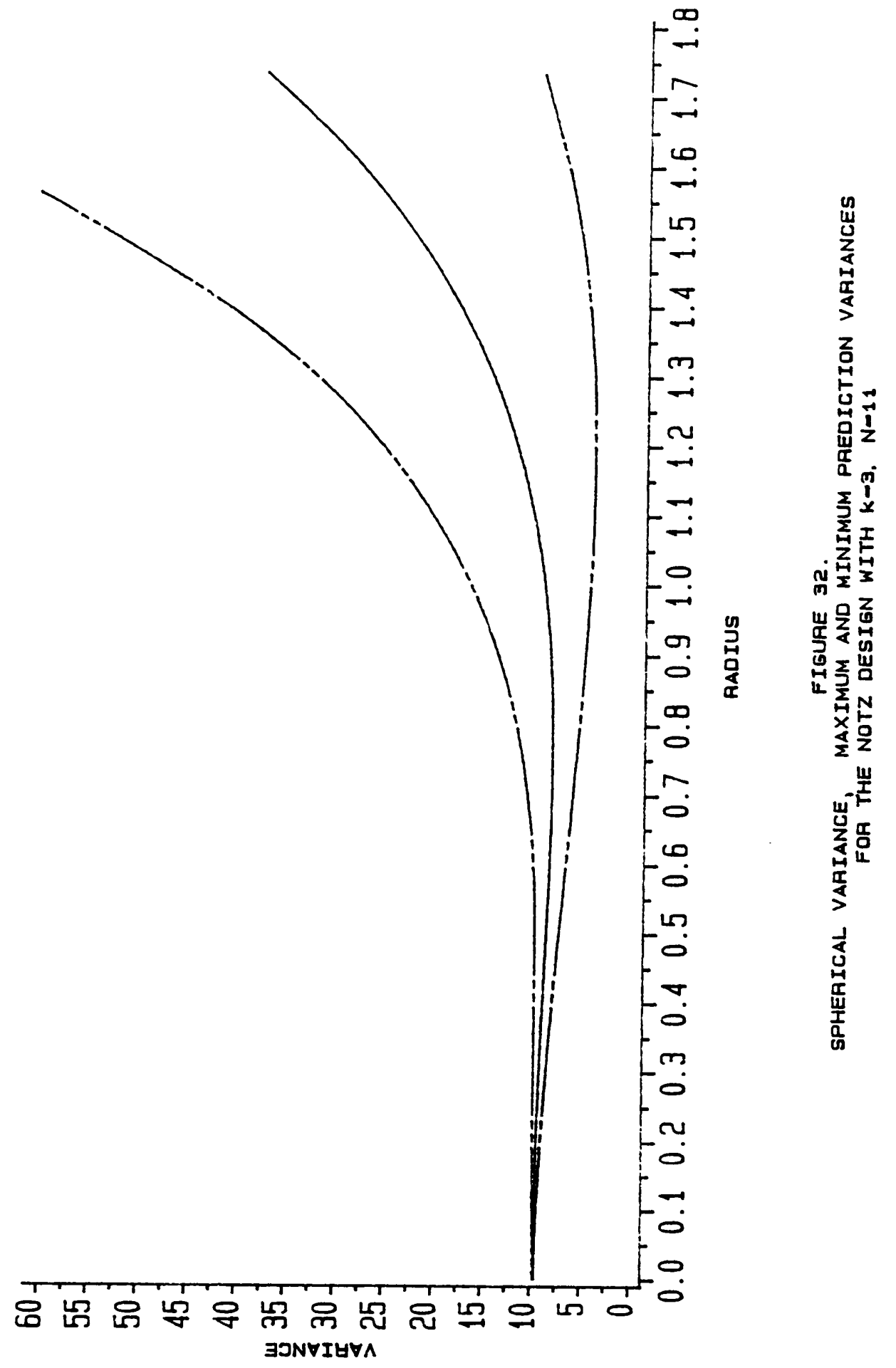


the same D-efficiency, has notably higher spherical and maximum prediction variances on spheres in the region. It is gratifying to note that Welch's compromise design, WC10, which resulted from an effort to improve the prediction variances over the design points, yields a better overall prediction variance picture than the IVD10 design.

Comparisons of the average prediction variances on spheres for the MB10 and WD10 designs may be derived from Figure 33. The spherical variance curve obtained with the WC11 design is also plotted there. Plots depicting the more complete behavior of the prediction variances on spheres are in Figure 34 for MB10, Figure 35 for WD10, and in Figure 36 for WC11.

The WC11 design has a D-efficiency of $94.4 \%$. Although WC11 has a higher D-efficiency than the ten point designs, its predicting ability is not as good in general. The MB10 design, and the WC10 design as well, in particular, have better overall prediction variance pictures and still have reasonable D-efficiencies.

In this and the preceding section it has been shown that designs with the same D-efficiencies do not necessarily possess the same ability to estimate the response. Nor is a design with a high D-efficiency relative to competing designs guaranteed to produce more precise estimates of the response or better behavior of the prediction variances on spheres throughout the region. The Defficiency criterion was not developed to be a measure of the prediction capability of a design. Obviously, from the illustrations presented here, it should not be used as one. The plots of the spherical variance and maximum and minimum prediction variances on spheres throughout a region very ably depict the nature of the prediction variances for a design. 


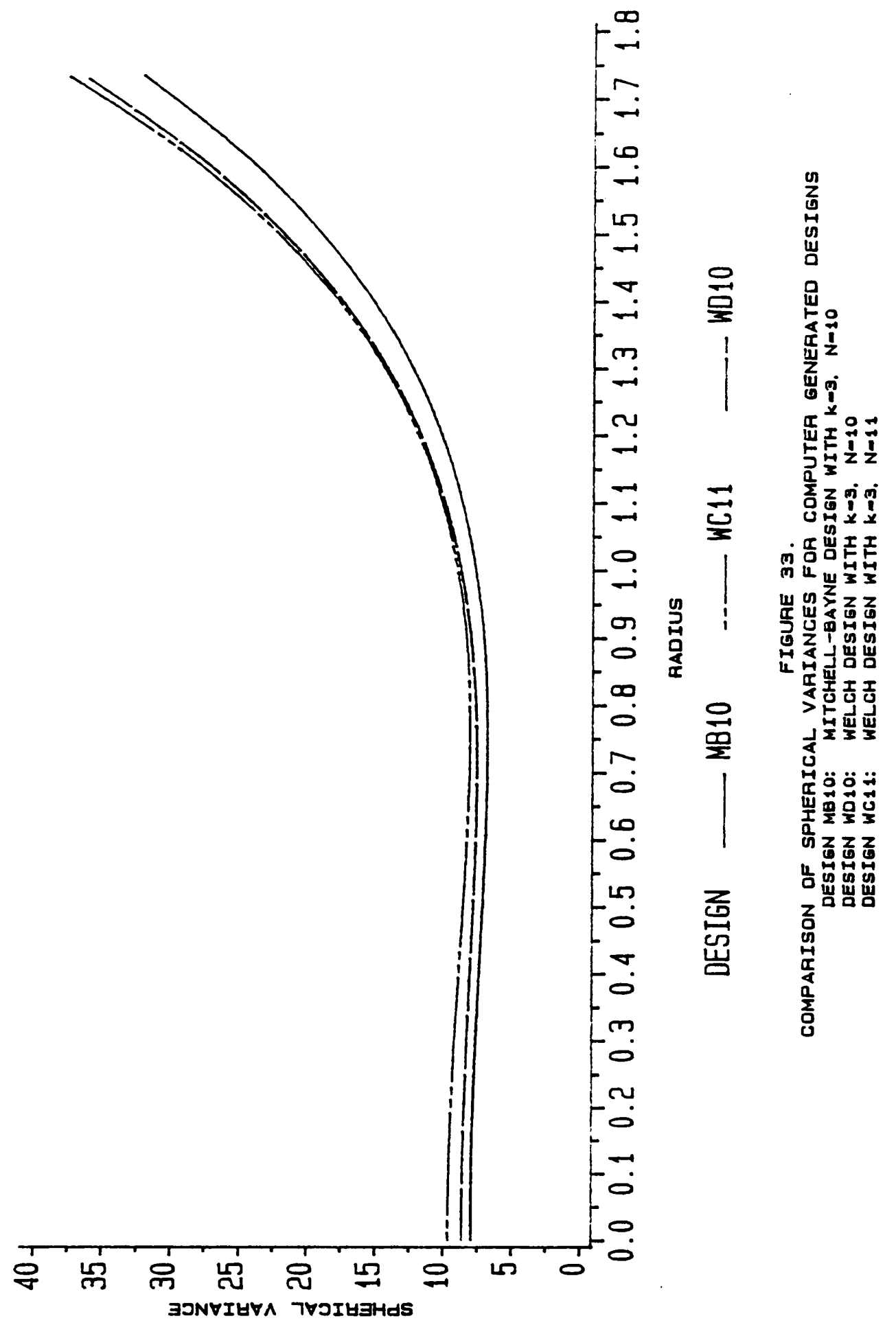




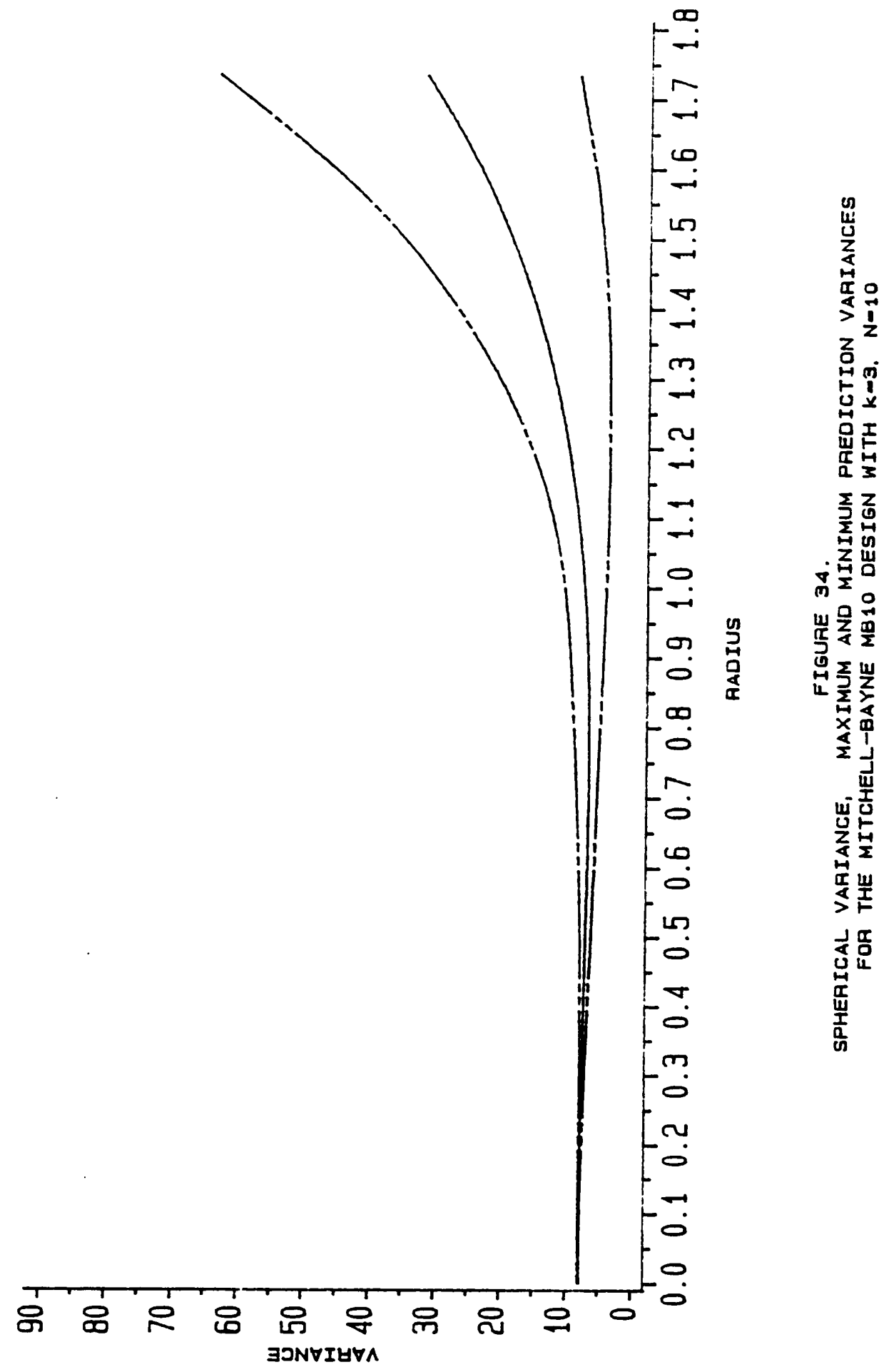




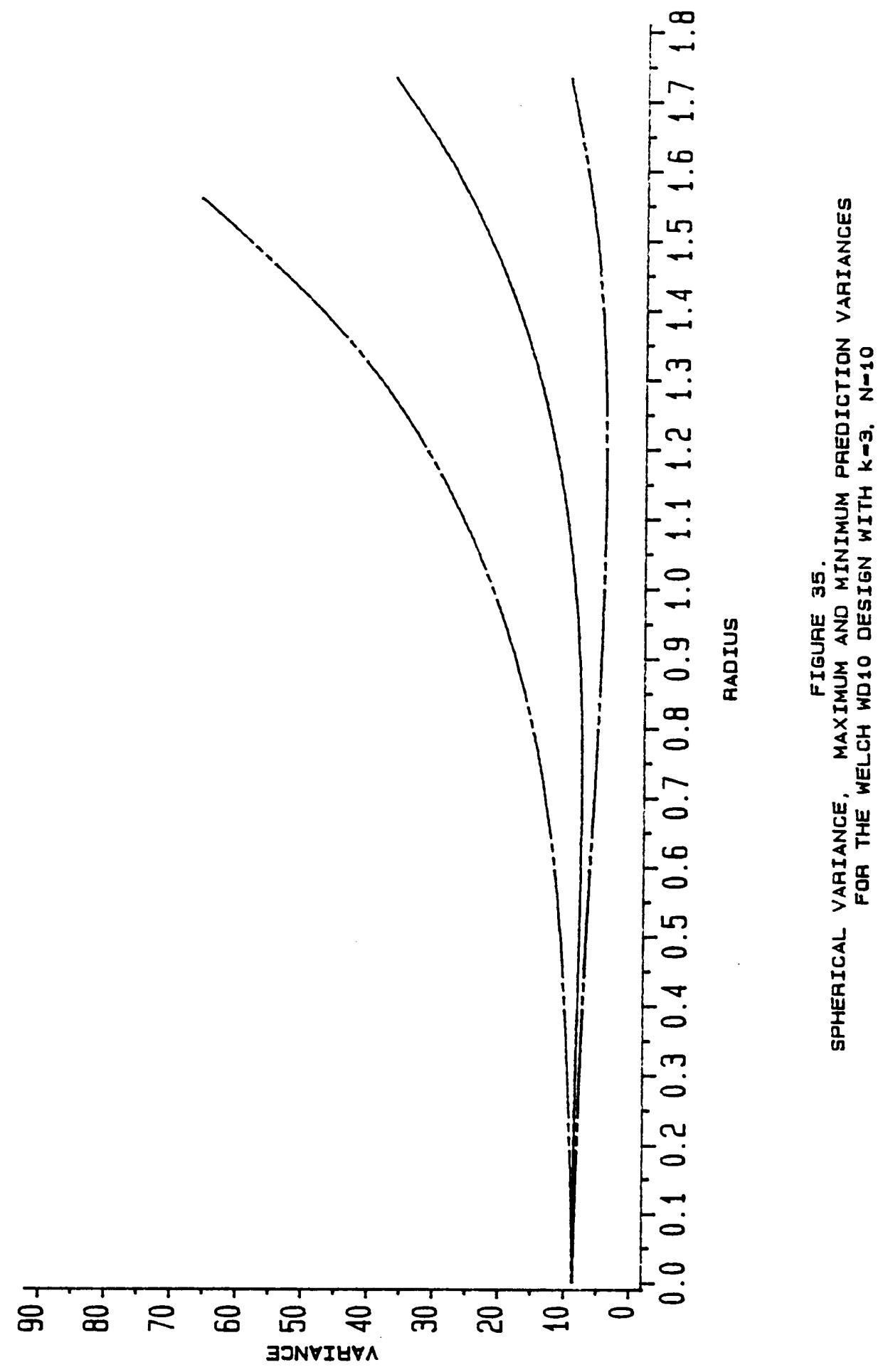




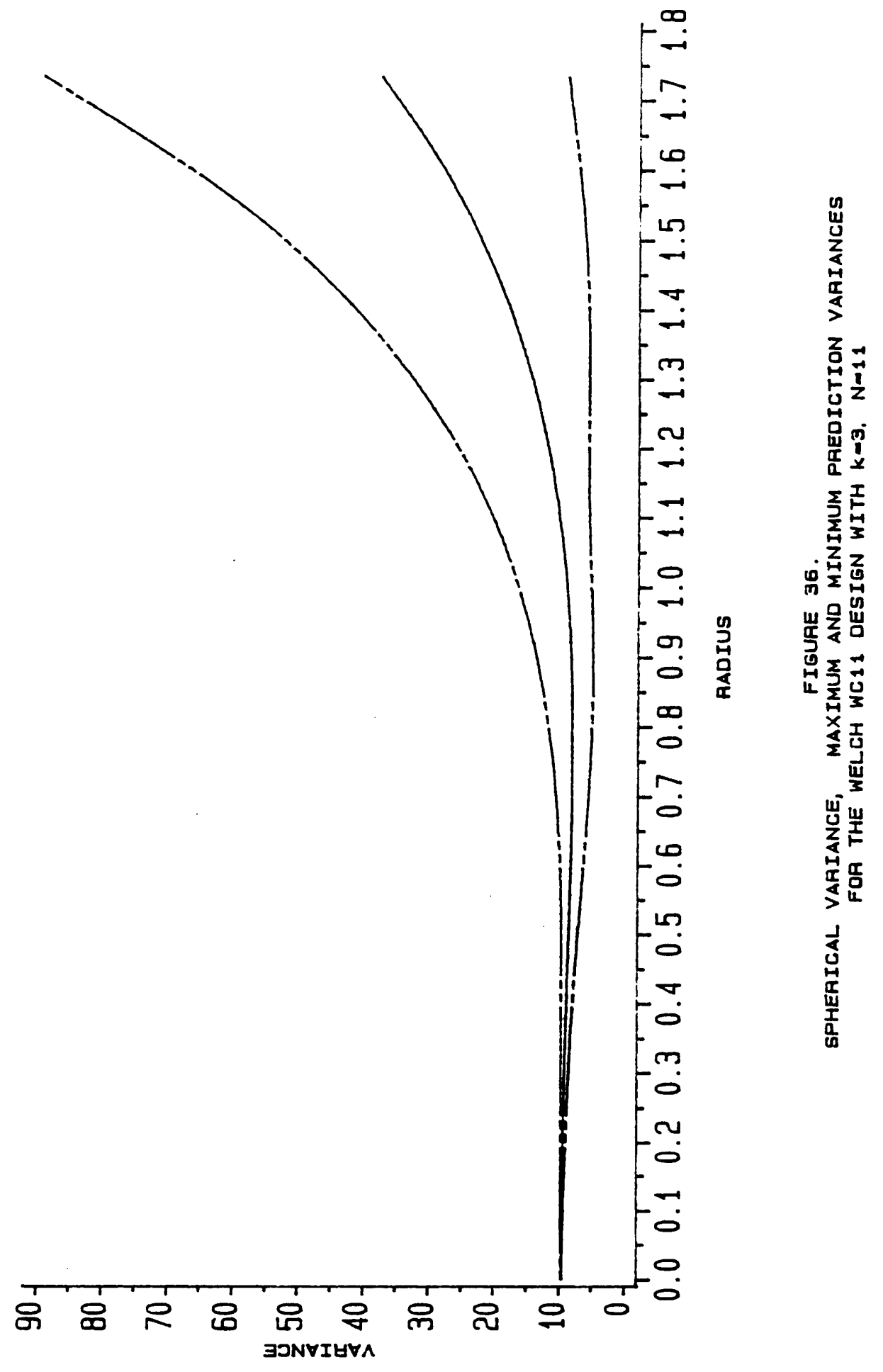




\subsection{Measuring Deviation from Rotatability}

Throughout the illustrations of this chapter, the dispersion in the prediction variances on spheres has been discussed. The amount of dispersion in the variances on a sphere indicates how consistent the precision of the estimated responses is on the sphere. Since the dispersion in the prediction variances on a sphere measures the similarity of the variances on that sphere, it is reasonable to use a measure of the dispersion to guage the deviation from rotatability for a design. Thus, the RofV will be used in this section as a means of determining how near a design is to possessing the rotatability property of equal prediction variances on spheres.

The ranges of the prediction variances on spheres about the design center will be plotted for the $3^{2}$ factorial design and the three variable hybrid designs of Roquemore (1976). These designs are presented in the papers by Khuri (1988) and Draper and Guttman (1988) to illustrate their indices of rotatability, (see section 2.3.4). The conclusions drawn from the plots representing the dispersion in the prediction variances on spheres will be compared with the results obtained with the rotatability indices of Khuri and of Draper and Guttman where applicable.

\subsubsection{The Deviation from Rotatability of a $3^{2}$ Factorial Design}

The ranges of the prediction variances on spheres obtained with a $3^{2}$ factorial design are depicted in Figure 37. The prediction variances for the design in two variables have been considered on spheres of radii ranging from $r=0$ to $r=\sqrt{2}=1.4142$. Note that the variances have not been weighted by the size of the design.

The RofV on a sphere is the distance between the maximum and minimum prediction variance curves at the radius of the sphere. For example, on a sphere of radius 1.40 the maximum 


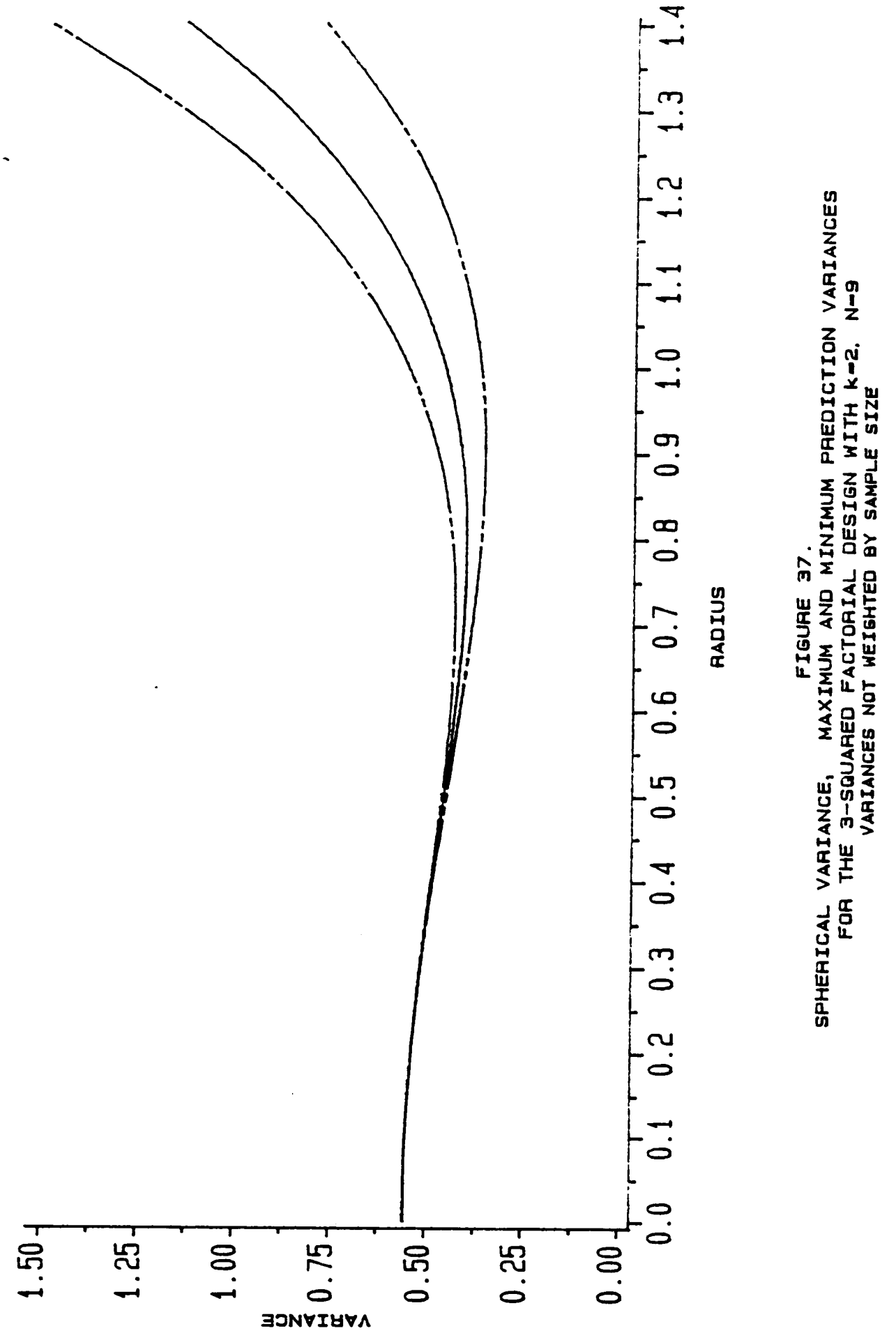


prediction variance occurs at $x_{1}= \pm 1.40, x_{2}=0$ and is equal to 1.496 ; the minimum prediction variance at $x_{1}= \pm 0.98995, x_{2}= \pm 0.98995$ is 0.776 . Thus, $\operatorname{RofV}(r=1.40)$ is $1.496-0.776=0.72$ which is relatively large considering the size of the prediction variances throughout the region. The locations of the maximum and minimum prediction variances were generated by the application of the MINOS optimization algorithm specifying $r=1.40$ for this design.

Beyond a radius of about 1.0 the prediction variances on a sphere appear to be far from equal, indicating that the design is not close to being rotatable on those spheres. It should be noted that the increased dispersion observed on the outer spheres of the region may partially be an artifact of the larger prediction variances which occur in that part of the region. Obviously, as the variances get larger the observed variability among them will naturally increase. However, for the nonrotatable designs considered in this research, the RofV was always observed to increase as the radius increased. Thus, the variances on a sphere on the outskirts of the region are expected to be more dispersed than the variances on a sphere closer to the design center. The researcher should be cautioned to take into account the magnitude of the prediction variances when considering their dispersion.

Khuri found the $3^{2}$ factorial design to be $93.08 \%$ rotatable with his measure of rotatability. Although the prediction variances on a sphere are nearly equal when the radius of the sphere is small, it is apparent from Figure 37 that there is a fair amount of dispersion among the variances on larger spheres. In light of this, Khuri's assessment of the degree to which the $3^{2}$ factorial design possesses the rotatability property seems a little high. Unfortunately, there are no guidelines available to indicate how near rotatable a design is which is measured to be $93.08 \%$ rotatable.

The index of rotatability due to Draper and Guttman is calculated to be $m=3.73$ for the $3^{2}$ factorial design. Based on the benchmark values for $m$ given in their work (given in Figure 3 of Draper and Guttman (1988)), this value correctly indicates that the outer prediction variance contour of the $3^{2}$ factorial is shaped like a square with rounded corners. Clearly, the outer prediction 
variance contour is very different from the outer variance contour of a rotatable design, which is a circle in two variables and is indicated by an index of rotatability of $m=2$. The index of rotatability indicates that the variances are not equal on the outer spheres of the region. This is the only information provided by Draper's and Guttman's index. To determine the disparity among the variances on a sphere passing through the outer contour, or any contour, it is necessary to look at the values of the other contours which pass through the sphere. The dispersion in the values of the prediction variance contours which pass through the sphere is the dispersion in the prediction variances on that sphere. The index of rotatability does not indicate the better behavior of the variances on the inner spheres of the region.

\subsubsection{The Deviation from Rotatability of Hybrid Designs}

The three variable hybrid designs constructed by Roquemore, and labelled as $310,311 \mathrm{~A}$ and 311B, are considered to be nearly rotatable designs. The dispersion in the prediction variances on spheres for the 310 design is produced in Figure 38. This particular 310 design has been centered to look at the behavior of the prediction variances on spheres about the design center. Also, the form of the design used here has ten design points and no center runs, unlike the 310 hybrid in section 5.2.1. The maximum and minimum prediction variance curves corresponding to the $311 \mathrm{~A}$ and $311 \mathrm{~B}$ designs are plotted in Figures 39 and 40 respectively. Both of these designs have 11 design points, one of which is a center point. Note that the variance axes of Figures 39 and 40 are on a larger scale than that of Figure 38. This was done to get a more detailed picture of the dispersion in the prediction variances for the $311 \mathrm{~A}$ and $311 \mathrm{~B}$ designs.

Clearly, the 310 hybrid design is the least rotatable of the three. Once again, the amount of dipersion on the outer spheres may not be as substantial as it appears due to the increased size of the variances on those spheres. The $311 \mathrm{~A}$ and $311 \mathrm{~B}$ designs have nearly equal prediction variances on all spheres throughout the region, indicating that they are very near to possessing the rotatability 


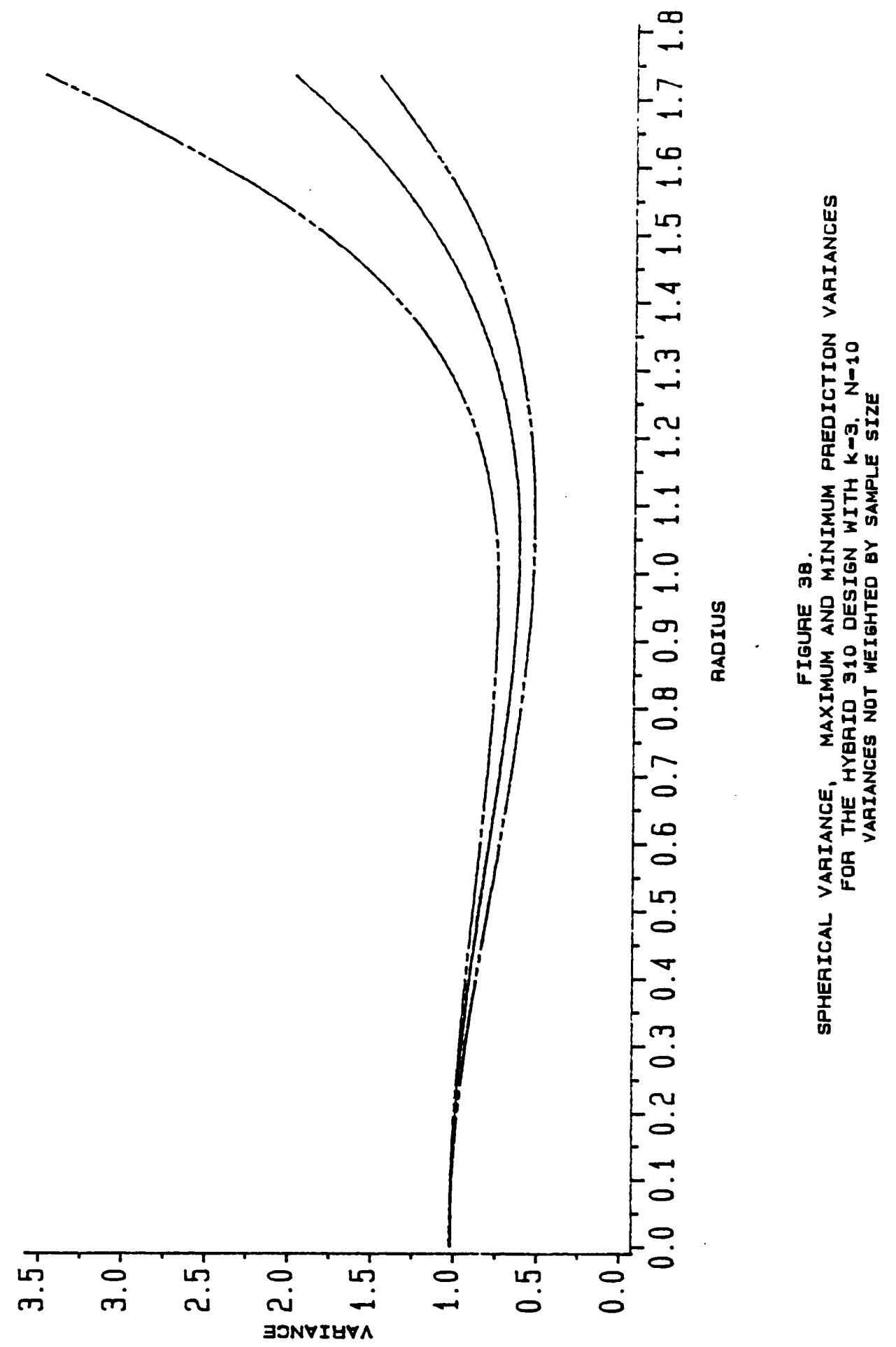




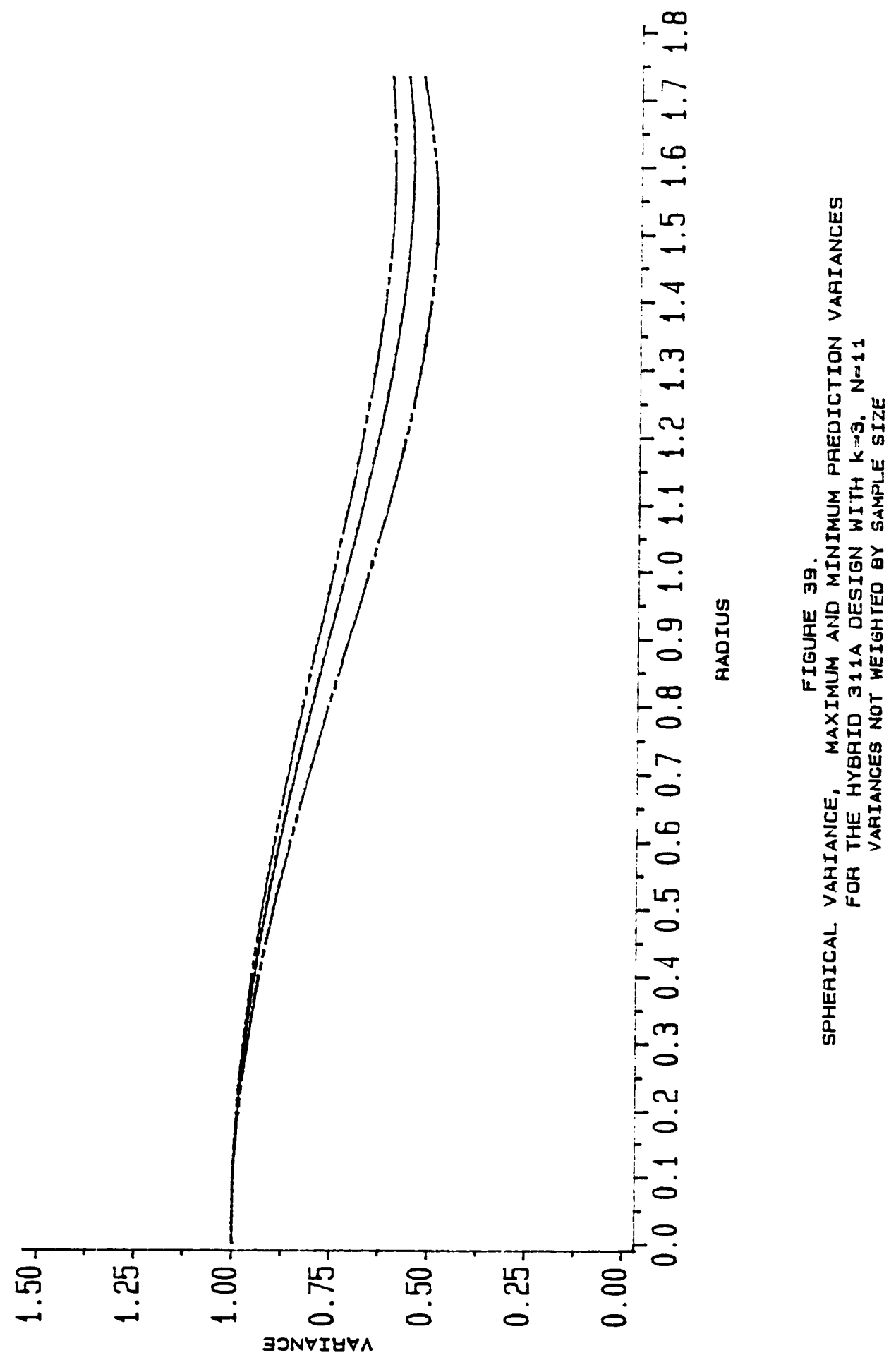




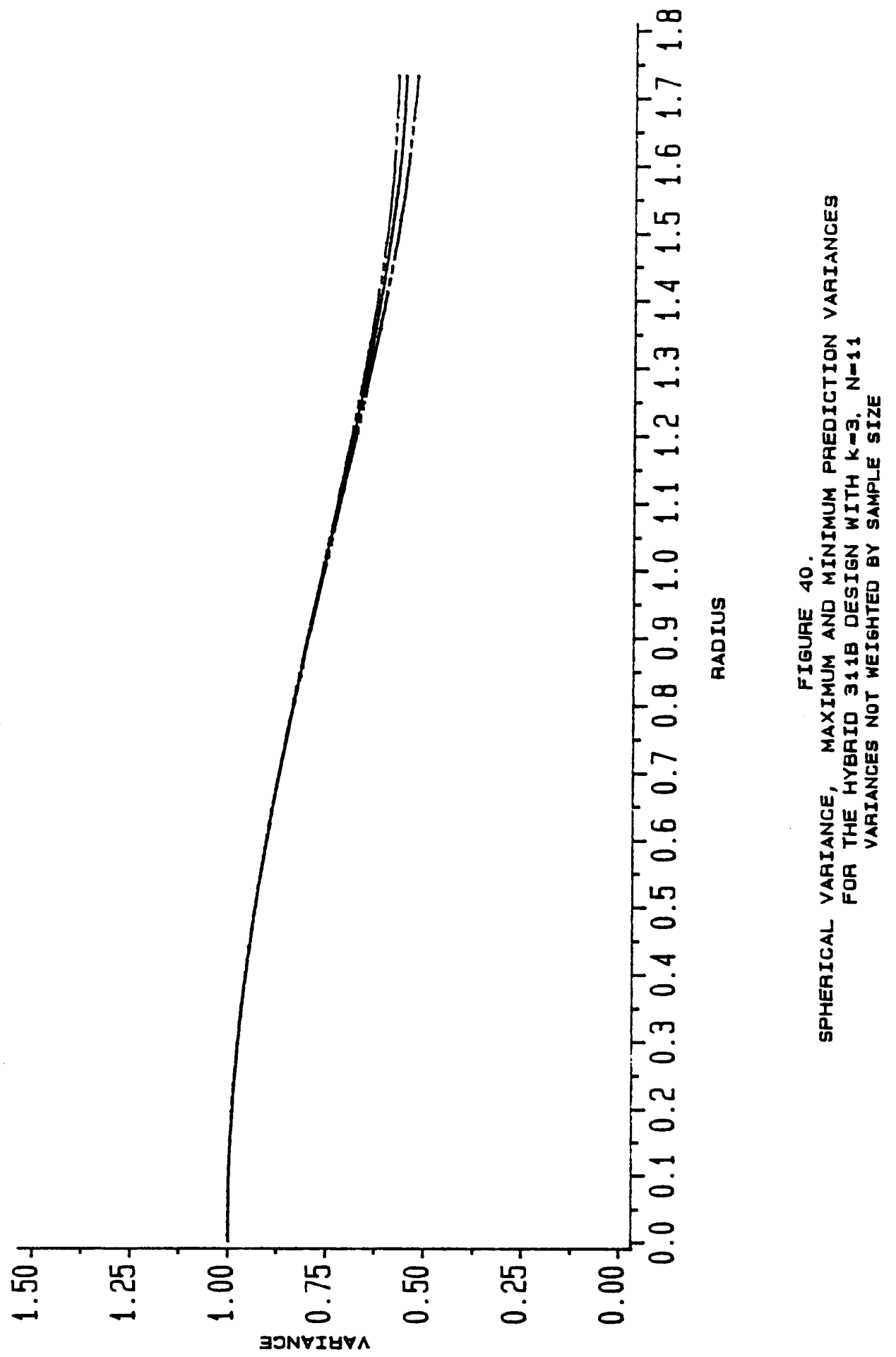


property. There is somewhat more dispersion in the prediction variances on spheres for the $311 \mathrm{~A}$ design than the $311 \mathrm{~B}$ design. This contradicts Roquemore's conclusion that the $311 \mathrm{~A}$ design is the most nearly rotatable of the three. His conclusion is based on the observation of the shape of the prediction variance contours, and, in particular, the outermost contour in the design region, for each design. It has been seen that the measurement of deviation from rotatability for a design via the shape of the contours alone can be misleading and may lead to erroneous conclusions about the consistency of the prediction variances on spheres for a design.

The application of Khuri's measure of rotatability to the hybrid designs finds the $310,311 \mathrm{~A}$ and $311 \mathrm{~B}$ designs to be respectively $94.89,99.40$ and 98.99 percent rotatable. It is felt that the percent rotatability measure for the 310 design is high considering the dispersion in the variances on the outer spheres of the region. The measures for the $311 \mathrm{~A}$ and $311 \mathrm{~B}$ designs appear reasonable. They are in keeping with the conclusion that the $311 \mathrm{~A}$ and $311 \mathrm{~B}$ designs are nearly rotatable. However, using Khuri's measure one would conclude that the $311 \mathrm{~A}$ is more near to possessing the rotatability property than the $311 \mathrm{~B}$ hybrid design.

Since the 310 and $311 \mathrm{~A}$ hybrid designs are not symmetric, that is, their third design moments are not all equal to zero, Draper's and Guttman's index of rotatability cannot be applied to these designs. For the symmetric $311 \mathrm{~B}$ design the index of rotatability is $\mathrm{m}=1.764$. This is quite close to the value of $m=2$, the index of rotatability for a rotatable design. Thus, the outer prediction variance contour of the $311 \mathrm{~B}$ design is nearly spherical in shape. The implication is that the $311 \mathrm{~B}$ hybrid design is a nearly rotatable design.

The indices of rotatability introduced by Khuri and by Draper and Guttman do not actually measure the dispersion in the prediction variances on spheres. Instead, they attempt to quantify the difference between certain characteristics of a non-rotatable design, specifically the design moments and shape of the prediction variance contours, and the corresponding characteristics of a rotatable design. They are artificial measures of deviation from rotatability. 
The RofV, on the other hand, truly measures the dispersion in the prediction variances on spheres. Plots of the maximum and minimum prediction variance functions enable the depiction of the dispersion in the variances across all spheres in a specified region. From the plots a comprehensive description of the deviation from rotatability of a design may be obtained. A singlevalued measure of deviation from rotatability, such as those introduced by Khuri and by Draper and Guttman, can not satisfactorily characterize the dispersion in the prediction variances for a design in this fashion. Nor can such measures indicate the quality of prediction for a design which is one of the features of the picture of prediction capability generated with the graphical method presented in this thesis. 


\section{Chapter VI}

\section{Concluding Remarks and Further Research}

\subsection{Concluding Remarks}

The estimation of the response is an important consideration in the exploration of a response surface. It is reasonable to look at the quality of prediction on spheres in order to assess the overall prediction capability of a design. The spherical variance and the prediction variance dispersion measures provide a comprehensive description of the behavior of the prediction variances on a sphere. Plots of the spherical variance and maximum and minimum prediction variance functions against the radius of the sphere permit an honest assessment of the prediction capability of a design in some region. The plots give an overview of the changing prediction variances in the region that cannot be achieved with a single measure of prediction capability. A record of the locations of maximum and minimum prediction variances on spheres throughout the region gives the researcher knowledge of where prediction is at its worst and best. This knowledge can be used to advantage in the interpretation of results and the planning of the next step in the exploration of the response surface. 
The plots can be used to examine the effects on the prediction variances of a change in the design plan, whether it be unforseen, such as the loss of a design point, or planned, such as the augmentation of a design. Deviation from rotatability for a design can be measured from the maximum and minimum variance curves representing the range in the variances on spheres. The graphical representation of $V^{r}$ and the maximum and minimum prediction variances also allows for the easy comparison of competing designs. The plots may be used as the sole basis or in conjunction with other design criteria to select an experimental design.

Naturally, there are many factors to be considered in the assessment of the prediction capability of a design in a given experimental situation. Whether the picture of the behavior of the prediction variances is acceptable or not is a judgement to be made by the researcher. The spherical variance and prediction variance dispersion functions and their graphs provide a useful means of assessing the quality of the estimated responses on a sphere and throughout the region.

\subsection{Directions for Further Study}

The principal goal of this research was to introduce graphical methods of assessing the prediction capability of an experimental design. In particular, it was desired to display the behavior of the prediction variances at locations which are the same distance from the center of the region under consideration. The spherical variance and PVD measures are a most satisfactory result of this endeavor. This research project has also produced several directions for further study in this area.

Trends in the spherical variance and PVD measures for the second order model case need to be further investigated. From the illustrations of Chapter V, it is believed that, in most cases, the spherical variance, and the maximum and minimum prediction variance functions, decrease from a radius of $r=0$ to some minimum value, and then increase steadily beyond that point. It would 
be of interest to determine the relationship between the radius at which the spherical variance begins climbing and the position of the design points in the region.

For certain designs the RofV and VofV are not necessarily invariant to the number of center points of the design. When setting up an experimental plan, it would be useful to know if the dispersion in the variances on spheres could be appreciably improved with the addition of a few center points to such a design. To this end, a study of the effects of the addition of center points to designs which do not satisfy the conditions of Theorems 4.2 .4 or 4.2 .5 is warranted.

Some designs, such as the designs of sections 5.2.2 and 5.2.3, were constructed to predict at locations on or within the unit cube. The consideration of the prediction variances on spheres within the cube can be accomplished as described in this thesis. In the illustrations of sections 5.2.2. and 5.2.3 the spherical variance and the maximum and minimum prediction variances on spheres were computed under the assumption that it was of interest to predict at all locations in a spherical region. However, if the response is to be estimated only at locations on spheres of radius greater than 1 which lie on or within the unit cube, the computation of the variance functions should be modified to reflect the cuboidal region of interest. It would be a simple matter to modify the MINOS algorithm to obtain the optimal prediction variances at locations on or within the unit cube. The modification of the form of the spherical variance, however, will be more difficult.

The most obvious extension to the results presented here is to derive forms for the spherical variance and PVD measures for other models besides first and second order polynomials. Also, it would be useful to develop forms for the prediction variance functions under more general assumptions on the error term of the model or different methods of estimation of the model parameters, such as ridge regression estimation.

The forms of the prediction variance functions depend on the assumed model. A measure which describes the bias in the estimated responses at locations on a sphere induced by inadequate specification of the model would provide a very informative complement to the spherical variance 
and PVD measures. Together, these measures could be used to develop comprehensive pictures of the prediction capability and robustness to model misspecification for a design.

A broad area of further study to which the ideas developed here could be applied is design augmentation. It will be necessary to create an algorithm to implement the spherical variance criterion presented in section 4.3. There are other criteria which might be used to select a point to be added to a design. For example, a criterion which selects the new point in order to minimize some function of the bias, or, perhaps, more appropriately, the mean square error of prediction, at locations in a region would merit development and further investigation. 


\section{References}

Aitken, A. C. (1948), Determinants and Matrices, Oliver \& Boyd, London.

Atkinson, A. C. (1973), "Multifactor Second Order Designs for Cuboidal Regions," Biometrika, $60,15-19$.

Atwood, C. L. (1969), "Optimal and Efficient Designs of Experiments," Annals of Mathematical Statistics, 40, 1570-1602.

Box, G. E. P. (1954), "The Exploration and Exploitation of Response Surfaces: Some General Considerations and Examples," Biometrics, 10, 16-60.

Box, G. E. P. (1982) "Choice of Response Surface Design and Alphabetic Optimality," Utilitas Math., 21B, 11-55.

Box, G. E. P. and Behnken, D. W. (1960), "Some New Three-Level Designs for the Study of Quantitative Variables," Technometrics, 2, 455-475.

Box G. E. P., and Draper, N. R. (1959), "A Basis for the Selection of a Response Surface Design," J. Amer. Statistical Assoc., 54, 622-654. (1963), "The Choice of a Second Order Rotatable Design," Biometrika, 50, 335-352.

Box, G. E. P., and Hunter, J. S. (1957), "Multifactor Designs for Exploring Response Surfaces," Annals of Mathematical Statistics, 28, 195-241.

Box, G. E. P. and Wilson, K. B. (1951), "On the Experimental Attainment of Optimum Conditions," Journal of the Royal Statistical Society, B, 13, 1.

Box, G. E. P., and Youle, P. V. (1955), "The Exploration and Exploitation of Response Surfaces: An Example of the Link Between the Fitted Surface and the Basic Mechanism," Biometrics, $11,287-323$.

Box, M. J. and Draper, N. R. (1971), "Factorial Designs, the $\left|X^{\prime} X\right|$ Criterion, and Some Related Matters," Technometrics, 13, 731-742. 
(1974), "On Minimum-Point Second Order Designs," Technometrics, 16, 613-616.

Draper, N. R. (1963), "Ridge Analysis of Response Surfaces," Technometrics, 5, 469-479.

Draper, N. R. (1982), "Center Points in Second-Order Response Surface Designs," Technometrics, 24, 127-133.

Draper, N. R. and Guttman, I. (1988), "An Index of Rotatability," Technometrics, to appear February, 1988.

Dykstra, O., Jr. (1971), "The Augmentation of Experimental Data to Maximize $\left|X^{\prime} X\right|$, , Technometrics, 13, 682-688.

Gaylor, D. W. and Merrill, J. A. (1968), "Augmenting Existing Data in Multiple Regression," Technomentrics, 10, 73-81.

Graybill, F. A. (1976), Theory and Application of the Linear Model, Duxbury Press, North Scituate, Massachusetts.

Hebble, T. L., and Mitchell, T. J. (1972), "Repairing Response Surface Designs," Technometrics, $14,767-779$.

Hoke, A. T. (1974), "Economical Second-Order Designs Based on Irregular Fractions of the $3^{n}$ Factorial," Technometrics, 16, 375-384.

Hussey, J. R. (1983), Effects of Correlated Induction Schemes on Variance Criteria in Experimentally Designed Computer Simulations, unpublished doctoral dissertation, Virginia Polytechnic Institute and State University, Blacksburg, Virginia.

Hussey, J. R., Myers, R. H., and Houck, E. (1987), "Correlated Simulation Experiments in First Order Response Surface Designs," Operations Research, to appear.

Khuri, A. I. (1988), "A Measure of Rotatability for Response Surface Designs," Technometrics, to appear February, 1988.

Kiefer, J., and Wolfowitz, J. (1959), "Optimum Designs in Regression Problems," Annals of Mathematical Statistics , 30, 271-294. (1960), "The Equivalence of Two Extremum Problems," Canad. J. Math., 12, 363-366.

Lucas, J. M. (1976), "Which Response Surface Design is Best," Technometrics, 18, 411-417.

Mitchell, T. J. (1974), "An Algorithm for the Construction of D-optimal Experimental Designs," Technometrics, 16, 203-210.

Mitchell, T. J. and Bayne, C. K. (1978), "D-optimal Fractions of Three-Level Factorial Designs," Technometrics, 20, 369-380.

Murtagh, B. A. and Saunders, M. A. (1983), "MINOS 5.1 User's Guide," Technical Report SOL 83-20R Department of Operations Research, Stanford University, Stanford, California.

Myers, R. H. (1976), Response Surface Methodology, distributed by Edwards Brothers, Inc., Ann Arbor, Michigan.

Nalimov, V. V., Golikova, T. I., and Mikeshina, N. G. (1970), "On Practical Use of the Concept of D-Optimality," Technometrics, 12, 799-812. 
Notz, W. (1982), "Minimal Point Second Order Designs," Journal of Statistical Planning and Inference, 6, 47-58.

Rao, C. R. (1973), Linear Statistical Inference and Its Applications, Second Edition, John Wilcy and Sons, New York.

Roquemore, K. G. (1976), "Hybrid Designs for Quadratic Response Surfaces," Technometrics, 18, $419-423$.

St. John, R. C. and Draper, N. R. (1975), "D-Optimality for Regression Designs: A Review," Technometrics, 17, 15-23.

Stroud, A. H. (1971), Approximate Calculation of Multiple Integrals, Prentice-Hall, Englewood Cliffs, New Jersey.

Swokowski, E. W. (1979), Calculus with Analytic Geometry, Second Edition, Prindle, Weber \& Schmidt, Boston.

Welch, W. J. (1982), "Branch-and-Bound Search for Experimental Designs Based on D-Optimality and Other Criteria," Technometrics, 24, 41-48. 


\section{Appendix A}

\section{Appendices for Chapter III}

\section{Appendix A.1}

\section{Optimization of the Prediction Variance Under Case I for $k=2$ Variables}

Suppose that $0<\lambda_{1}<\lambda_{2}$. We wish to find values of $z_{1}$ and $z_{2}$ which optimize the function given by (6.1) with $\mathrm{k}=2$ under the constraint that $z_{1}^{2}+z_{2}^{2}=r^{2}$. For ease of notation, write

$$
L\left(z_{1}, z_{2}\right)=\frac{1}{N}+\lambda_{1} z_{1}^{2}+\lambda_{2} z_{2}^{2}
$$

The constraint implies that $z_{1}^{2}=r^{2}-z_{2}^{2}$, and, thus, we may write

$$
L\left(z_{1}, z_{2}\right)=\frac{1}{N}+\left(\lambda_{2}-\lambda_{1}\right) z_{2}^{2}+\lambda_{1} r^{2}
$$


Since $\left(\lambda_{2}-\lambda_{1}\right)>0$ we see that to maximize (A.1.1) $z_{2}^{2}$ should be made as large as possible (see Figure A.1). Under the constraint the largest possible value of $z_{2}^{2}$ is $r^{2}$; that is, $z_{2}= \pm r$, will maximize $\mathrm{L}$. Thus, $z_{1}=0$ to satisfy the constraint. The maximum value of $\mathrm{L}$ is then

$$
L\left(z_{1}=0, z_{2}= \pm r\right)=\frac{1}{N}+\lambda_{2} r^{2}
$$

where $\lambda_{2}$ is the largest eigenvalue of $\left(X^{\prime} X\right)^{-1}$.

Conversely, to minimize $L\left(z_{1}, z_{2}\right)$ the weight of $z_{2}^{2}$ should be made as small as possible. To do this, take $z_{2}=0$. The condition that $z_{1}^{2}+z_{2}^{2}=r^{2}$ gives $z_{1}= \pm r$. The minimum value of $L\left(z_{1}, z_{2}\right)$ occurs for $z_{1}= \pm r$ and $z_{2}=0$ and is

$$
L\left(z_{1}= \pm r, z_{2}=0\right)=\frac{1}{N}+\lambda_{1} r^{2}
$$

where $\lambda_{1}$ is the smallest eigenvalue of $\left(X^{\prime} X\right)^{-1}$.

If $\lambda_{1}=\lambda_{2}$ then $L\left(z_{1}, z_{2}\right)$ is a constant for $z_{1}^{2}+z_{2}^{2}=r^{2}$, (see Figure A.2). In this case, the . maximum and minimum values of $\mathrm{L}$ are trivially the same. 


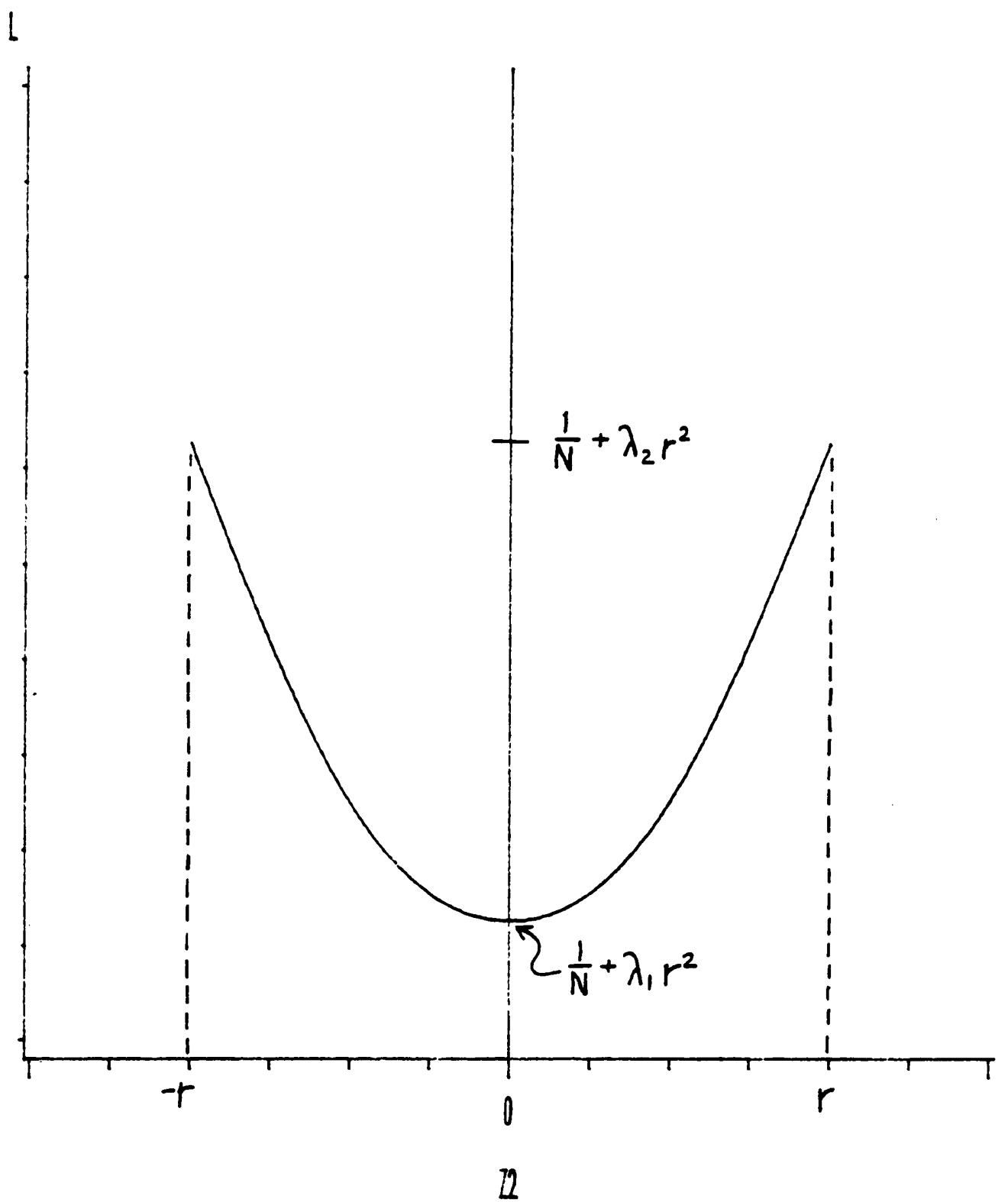

FIGPRE 1.1

$\lambda_{1} \lambda_{2}$ 


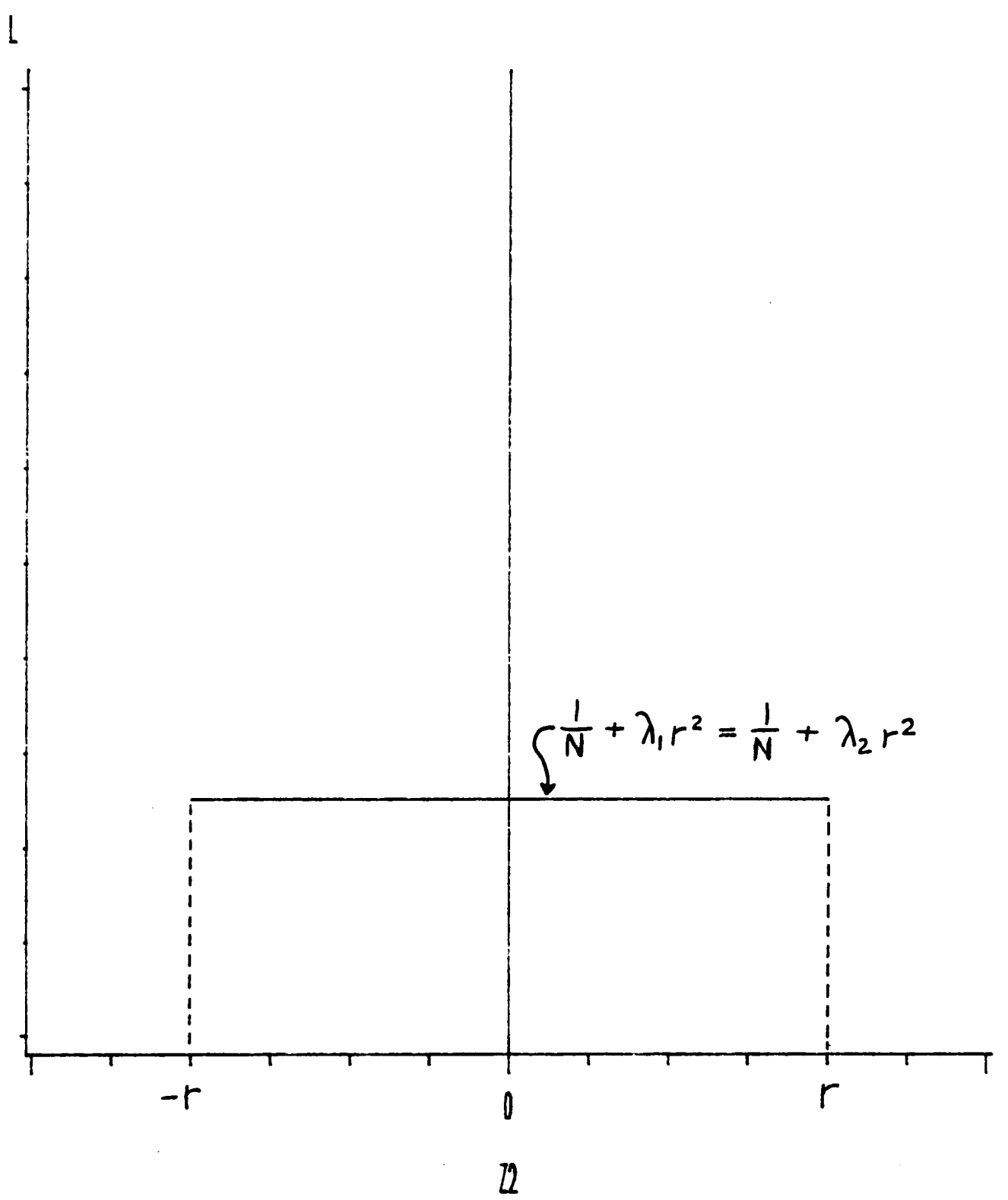

FlGPR 1.2

$\lambda_{1}=\lambda_{2}$ 


\section{Appendix A.2}

\section{Optimization of the Prediction Variance Under Case II}

Under the formulation of Case II in section 3.2.3, the optimization of $\operatorname{Var}(\hat{y}(\underline{x})) / \sigma^{2}$ on the surface of a hypersphere of radius $r$ centered about the point $\underline{x}=\underline{0}$ is equivalent to the optimization of $\operatorname{Var}(\hat{y}(\underline{w})) / \sigma^{2}$ subject to $\underline{w}=\underline{x}-\underline{h}$ being on a sphere of radius $r$ centered about $\underline{w}=\underline{0}-\underline{h}$. The solution to this constrained optimization problem may be found using the method of Lagrangian multipliers by solving the system of simultaneous equations defined by equation (3.2.9). The development of the solutions to (3.2.9) is presented in this section.

Let $Q$ be as defined in (3.2.9) and $\mu$ represent the Lagrangian multiplier. By (3.2.4), $Q$ can be expressed as

$$
Q=\frac{1}{N}+\underline{z}^{\prime} \Lambda \underline{z}-2 \underline{z^{\prime}} \Lambda \underline{m}+\underline{m}^{\prime} \Lambda \underline{m}-\mu\left(\underline{z}^{\prime} \underline{z}-r^{2}\right)
$$

where $\underline{z}=\left(\begin{array}{llll}z_{1} & z_{2} & \ldots & z_{k}\end{array}\right)^{\prime}=P^{\prime} \underline{x}=P^{\prime}(\underline{w}+\underline{h})$ and $\underline{m}=\left(\begin{array}{llll}m_{1} & m_{2} & \ldots & m_{k}\end{array}\right)^{\prime}=P^{\prime} \underline{h}$. The $k \times k$ matrix $P$ is the orthogonal matrix which decomposes the matrix $\left(W^{\prime} W\right)^{-1}$ into the diagonal matrix $\Lambda$ containing the positive eigenvalues of $\left(W^{\prime} W\right)^{-1}$.

It is convenient to write the set of partial derivatives $\frac{\partial Q}{\partial w_{1}}, \frac{\partial Q}{\partial w_{2}}, \ldots, \frac{\partial Q}{\partial w_{k}}$ in matrix form as

$$
\frac{\partial Q}{\partial \underline{w}}=\left(\frac{\partial Q}{\partial w_{1}} \frac{\partial Q}{\partial w_{2}} \ldots \frac{\partial Q}{\partial w_{k}}\right)^{\prime}
$$


(see Graybill (1976)) for some definitions and rules of matrix derivatives). By the chain rule for derivatives and with $\frac{\partial \underline{x}}{\partial \underline{w}}=\frac{\partial(\underline{w}+\underline{h})}{\hat{\partial} \underline{w}}=I$, the identity matrix, (3.2.9) is equivalent to

$$
\frac{\partial Q}{\partial \underline{w}}=\frac{\partial \underline{x}}{\partial \underline{w}} \cdot \frac{\partial Q}{\partial \underline{x}}=\frac{\partial Q}{\partial \underline{x}}=\underline{0}
$$

and $\frac{\partial Q}{\partial \mu}=0$.

Now, for $\underline{z}=P^{\prime} \underline{x}$ and $P$ an orthogonal matrix,

$$
\frac{\partial Q}{\partial \underline{x}}=\frac{\partial \underline{z}}{\partial \underline{x}} \cdot \frac{\partial Q}{\partial \underline{z}}=P^{\prime} \frac{\partial Q}{\partial \underline{z}}=0
$$

implies that

$$
\frac{\partial Q}{\partial \underline{z}}=\underline{0}
$$

Thus, equation (A.2.1) is satisfied by the solution to the equations

$$
\begin{aligned}
\frac{\partial \underline{Q}}{\partial \underline{z}} & =2 \Lambda \underline{z}-2 \Lambda \underline{m}-2 \mu \underline{z} \\
& =2(\Lambda-\mu I) \underline{z}-2 \Lambda \underline{m} \\
& =0
\end{aligned}
$$

and $\frac{\partial Q}{\partial \mu}=\underline{z}^{\prime} \underline{z}-r^{2}=0$. For $\underline{m} \neq \underline{Q}$ (Case II), the solution vector, $\underline{z}_{0}$, is then

$$
\underline{z}_{0}=(\Lambda-\mu I)^{-1} \Lambda \underline{m}
$$

where $\mu$ is such that $\underline{z}_{0}^{\prime} z_{0}=r^{2}$. In terms of the $x$-variables, the solution to (A.2.1) and a stationary point of the prediction variance on the surface of a hypersphere with center at $\underline{x}=\underline{Q}$ is given by

$$
\begin{aligned}
\underline{x}_{0} & =P \underline{z}_{0}=P(\Lambda-\mu)^{-1} \Lambda \underline{m} \\
& =P\left(\frac{\lambda_{1}}{\lambda_{1}-\mu} m_{1} \frac{\lambda_{2}}{\lambda_{2}-\mu} m_{2} \quad \cdots \frac{\lambda_{k}}{\lambda_{k}-\mu} m_{k}\right)^{\prime} .
\end{aligned}
$$


Note that $\underline{x}_{0}^{\prime} \underline{x}_{0}=r^{2}$.

The status of the stationary point $\underline{z}_{0}$, and, hence, $\underline{x}_{0}$, depends on the matrix of second partial derivatives of $Q$. Let $H(\underline{z})$ represent the matrix of second partial derivatives evaluated at the point $\underline{z}$, for the problem expressed as in (A.2.2). Then,

$$
\begin{aligned}
& H(\underline{z})=2(\Lambda-\mu \Lambda) \\
& =2\left[\begin{array}{cccc}
\lambda_{1}-\mu & 0 & \ldots & 0 \\
0 & \lambda_{2}-\mu & \cdots & 0 \\
\cdot & & & \cdot \\
\cdot & & & \cdot \\
0 & 0 & \cdots & \lambda_{k}-\mu
\end{array}\right] .
\end{aligned}
$$

If $H\left(\underline{z}_{0}\right)$ is a negative definite matrix, then the stationary point will be a location of maximum prediction variance on the sphere. Conversely, the stationary point will represent a location of minimum prediction variance if the Hessian matrix $H\left(\underline{z}_{0}\right)$ is positive definite.

First consider the solution of (A.2.2.) to find the location(s) of the maximum prediction variance on a sphere of radius $r$. In order for $H\left(\underline{z}_{0}\right)$ to be negative definite the quadratic form

$$
\underline{t}^{\prime} H\left(\underline{z}_{0}\right) \underline{t}=\sum_{l=1}^{k}\left(\lambda_{i}-\mu\right) t_{i}^{2}
$$

must be less than zero for all $t=\left(\begin{array}{lllll}t_{1} & t_{2} & \ldots & t_{k}\end{array}\right)^{\prime} \neq 0$. Only values of $\mu$ which are larger than $\lambda_{k}$, the largest eigenvalue of $\left(W^{\prime} W\right)^{-1}$ satisfy the requirement for $H\left(z_{0}\right)$ to be a negative definite matrix. To verify this statement, suppose $\mu$ is such that $\lambda_{1}<\lambda_{2}<\cdots<\lambda_{k-1}<\mu<\lambda_{k}$ and let $\underline{t}^{*}=\left(\begin{array}{lllll}0 & 0 & \ldots & 0 & 1\end{array}\right)^{\prime}$. Then,

$$
\underline{t}^{* \prime} H\left(\underline{z}_{0}\right) \underline{t}^{*}=\left(\lambda_{k}-\mu\right)>0
$$

Thus, there exists a vector $\underline{t} \neq \underline{Q}$ for which $\underline{t}^{\prime} H\left(\underline{z}_{0}\right) \underline{t}$ is not less than zero. Therefore, $H\left(\underline{z}_{0}\right)$ is negative definite provided $\mu>\lambda_{k}$. Thus, $\underline{z}_{0}$, or $\underline{x}_{0}=P_{\underline{z}_{0}}$, is a point of maximum prediction variance on a sphere of radius $r$ if $\mu>\lambda_{k}$ and $\frac{\partial Q}{\partial \mu}=0$. The second condition on $\mu$ is equivalent to 


$$
\underline{z}_{0}^{\prime} \underline{z}_{0}=\sum_{i=1}^{k}\left(\frac{\lambda_{i}}{\lambda_{i}-\mu}\right)^{2} m_{i}^{2}=r^{2}
$$

Note that $\underline{x}_{0}^{\prime} \underline{x}_{0}=\underline{z}_{0}^{\prime} P^{\prime} P \underline{z}_{0}=\underline{z}_{0}^{\prime} \underline{z}_{0}=r^{2}$ since $P$ is an orthogonal matrix. Thus, $\underline{x}_{0}$ is a location of maximum prediction variance on a sphere of radius $r$.

Similarly, it can be shown that $H\left(\underline{z}_{0}\right)$ is a positive definite matrix for $\lambda_{i}>0$ for all $i=1,2, \ldots, k$ if and only if $\mu<\lambda_{1}$ the smallest eigenvalue of $\left(W^{\prime} W\right)^{-1}$. Consequently, $\underline{z}_{0}$, or $\underline{x}_{0}=P \underline{z}_{0}$, is a location of the minimum prediction variance on a sphere of radius $r$ if $\mu<\lambda_{1}$ and $\sum_{i=1}^{k}\left(\frac{\lambda_{i}}{\lambda_{i}-\mu}\right)^{2} m_{i}^{2}=r^{2}$.

Again the second condition on $\mu$ guarantees that $\underline{x}_{0}$ is a point on the sphere. 


\section{Appendix A.3}

\section{Description of the MINOS Optimization Algorithm}

The MINOS optimization package (Murtagh and Saunders (1983)) is a fortran-based algorithm. Familiarity with the fortran language is helpful but not necessary to use the package. The MINOS 5.1 version was used to solve the optimization problems in this thesis.

MINOS is designed to solve general constrained optimization problems. The objective function, the function to be optimized, may be linear or nonlinear in the variables. For the optimization of the prediction variance the objective function is a nonlinear function in the variables $x_{1}, x_{2}, \ldots, x_{k}$ of the form

$$
\underline{x}^{*}\left(X^{\prime} X\right)^{-1} \underline{x}^{*}
$$

For the second order model case, $\underline{x}^{*}=\left(\begin{array}{llllllllll}1 & x_{1} & \ldots & x_{k} & x_{1}^{2} & \ldots & x_{k}^{2} & x_{1} x_{2} & \ldots & x_{k-1} x_{k}\end{array}\right)^{\prime} . \quad\left(X^{\prime} X\right)^{-1}$ is a matrix of constants with respect to the optimization.

The constraints of the problem may be linear, nonlinear or both. In this case the variables are constrained to be a point on a sphere of radius $r$. That is, $\sum_{i=1}^{k} x_{i}^{2}=r^{2}$, a nonlinear constraint.

To solve the problem of optimizing a nonlinear objective function subject to nonlinear constraints MINOS uses a projected augmented Lagrangian algorithm. The reader is referred to Murtagh and Saunders (1983) and the references listed there for a detailed description of this algorithm. Basically, through a series of iterations the algorithm attempts to optimize the objective function subject to linear constraints using a modified method of Lagrangian multipliers. The nonlinear constraints of the problem are replaced by linear approximations in this algorithm. 
To implement the MINOS package the user must input the form of the objective function and the first derivatives or gradients of the objective function. The constraint function and its first derivatives must also be specified. It is not necessary that functional forms for the first derivatives be known, however, the program will run more efficiently if they are given. The objective and constraint functions and their respective derivatives are specified in the MAIN program.

Other information about the problem is input into a separate file called the MPS file. In this file the number of variables in the problem, the type of optimization requested, that is, whether it is desired to maximize or minimize the function, and the value of the constraint, $r^{2}$ in this case, are specified. Initial values for the variables must be given in the MPS file. Bounds may be placed on the possible values of the variables here also. It is recommended that the variables be bounded to use the program since this often greatly improves the efficiency of the algorithm. Limits on the number of iterations used and other parameters of the algorithm may also be specified in the MPS file. The MINOS user's guide (Murtagh and Saunders (1983)) lists the parameters which the user may specify in the algorithm. Descriptions and examples of their use are found there as well. 


\section{Appendix $A .4$}

\section{Derivation of the Spherical Variance Under Case II}

The spherical variance under case II is developed in section 3.2.5. In this section the interim steps required for the derivation of $V^{r}$ in equation (3.2.14) are given.

Let $U_{r}=\left\{\underline{x}: \sum_{i=1}^{k} x_{i}^{2}=r^{2}\right\}$ denote the surface of a hypersphere of radius $r$ in the $x$-variables. In the $w$-axis system $U$, is represented as $U_{r}^{w}=\left\{\underline{w}: \sum_{i=1}^{k}\left(w_{i}+h_{i}\right)^{2}=r^{2}\right\}$. Further, let $\Psi \cdot 1=\int_{U_{r}} d \underline{x}$ and $\Psi_{w}^{-1}=\int_{U_{r}^{w}} d \underline{w}$ denote the surface of the hypersphere in the $x$-axis and $w$-axis systems respectively. Then, the average prediction variance on the surface of a hypersphere of radius $r$ centered about $\underline{x}=\underline{Q}$ is

$$
\begin{aligned}
V^{r} & =\frac{\Psi}{\sigma^{2}} \int_{U_{r}} \operatorname{Var}(\hat{y}(\underline{x})) d \underline{x} \\
& =\frac{\Psi_{w}}{\sigma^{2}} \int_{U_{r}^{w}} \operatorname{Var}(\hat{y}(\underline{w})) d \underline{w} .
\end{aligned}
$$

By (3.2.4)

$$
V^{r}=\Psi \int_{U_{r}}\left(\frac{1}{N}+\sum_{i=1}^{k} \lambda_{i} z_{i}^{2}-2 \sum_{i=1}^{k} \lambda_{i} m_{i} z_{i}+\sum_{i=1}^{k} \lambda_{i} m_{i}^{2}\right) d \underline{z}
$$

for $\underline{z}=P^{\prime} \underline{x}=P^{\prime}(\underline{w}+\underline{h})$. The transformation of $\underline{w}$ into $\underline{z}$ takes the surface $U_{r}^{w}$ into $U_{r}=\left\{z: \sum_{i=1}^{k} z_{i}^{2}=r^{2}\right\}$ and the surface area $\Psi_{w}^{-1}$ into $\Psi \cdot 1=\int_{U_{r}} d z$.

Before proceeding, recall that 


$$
\Psi \int_{U_{r}} z_{i} d \underline{z}=0
$$

and

$$
\Psi \int_{U_{r}} z_{i}^{2} d \underline{z}=\sigma_{2}=\frac{r^{2}}{k}
$$

is the second order spherical region moment as defined in section 3.2.3. Now, simplification of (A.4.1) yields

$$
\begin{aligned}
V^{r}= & \frac{1}{N} \Psi \int_{U_{r}} d \underline{z}+\sum_{i=1}^{k} \lambda_{i} \Psi \int_{U_{r}} z_{i}^{2} d \underline{z} \\
& -2 \sum_{i=1}^{k} \lambda_{i} m_{i} \Psi \int_{U_{r}} z_{i} d \underline{z}+\sum_{i=1}^{k} \lambda_{i} m_{i}^{2} \Psi \int_{U_{r}} d \underline{z} \\
= & \frac{1}{N}+\sigma_{2} \sum_{i=1}^{k} \lambda_{i}+\sum_{i=1}^{k} \lambda_{i} m_{i}^{2} \\
= & \frac{1}{N}+\frac{r^{2}}{k} \sum_{i=1}^{k} \lambda_{i}+\sum_{i=1}^{k} \lambda_{i} m_{i}^{2}
\end{aligned}
$$




\section{Appendix A.5}

\section{Derivation of the VofV Under Case II}

Using the notation set forth in Appendix A.4, the variance of the prediction variances on the surface of a hypersphere defined by equation (3.2.2) for case II can be expressed in the $w$-variables as

$$
\operatorname{Vof} V(r)=\Psi_{w} \int_{U_{p}^{w}}\left[\operatorname{Var}(\hat{y}(\underline{w})) / \sigma^{2}-V^{r}\right]^{2} d \underline{w}
$$

Application of the results of (3.2.4) and (3.2.14) and a transformation of variables yields

$$
\begin{aligned}
V o f V(r)= & \Psi \int_{U_{r}}\left[\left(\frac{1}{N}+\sum_{i=1}^{k} \lambda_{i} z_{i}^{2}-2 \sum_{i=1}^{k} \lambda_{i} m_{i} z_{i}+\sum_{i=1}^{k} \lambda_{i} m_{i}^{2}\right)\right. \\
& \left.-\left(\frac{1}{N}+\sigma_{2} \sum_{i=1}^{k} \lambda_{i}+\sum_{i=1}^{k} \lambda_{i} m_{i}^{2}\right)\right]^{2} d \underline{z} \\
= & \Psi \int_{U_{r}}\left[\sum_{i=1}^{k} \lambda_{i} z_{i}^{2}-2 \sum_{i=1}^{k} \lambda_{i} m_{i} z_{i}-\sigma_{2} \sum_{i=1}^{k} \lambda_{i}\right]^{2} d z .
\end{aligned}
$$

After expansion the integrand of (A.5.1) becomes

$$
\begin{aligned}
\sum_{i=1}^{k} \lambda_{i}^{2} z_{i}^{4} & +2 \sum_{i=1}^{k} \sum_{j=1}^{k} \lambda_{i} \lambda_{j} z_{i}^{2} z_{j}^{2}-2 \sigma_{2}\left(\sum_{i=1}^{k} \lambda_{i}^{2} z_{i}^{2}+\sum_{\substack{i=1 \\
i<j}}^{k} \sum_{j=1}^{k} \lambda_{i} \lambda_{j} z_{i}^{2}+\sum_{\substack{i=1 \\
i<j}}^{k} \sum_{j=1}^{k} \lambda_{i} \lambda_{j} z_{j}^{2}\right) \\
& -4\left(\sum \lambda_{i}^{2} m_{i}^{2} z_{i}^{3}+\sum_{i<j} \lambda_{i} \lambda_{j} m_{j} z_{i}^{2} z_{j}+\sum_{i<j} \lambda_{i} \lambda_{j} m_{i} z_{i} z_{j}^{2}\right) \\
& +4\left(\sum \lambda_{i}^{2} m_{i}^{2} z_{i}^{2}+2 \sum_{i<j} \lambda_{i} \lambda_{j} m_{i} m_{j} z_{i} z_{j}\right) \\
& +4 \sigma_{2}\left(\sum \lambda_{i}^{2} m_{i} z_{i}+\sum_{i<j} \lambda_{i} \lambda_{j} m_{i} z_{i}+\sum_{i<j} \lambda_{i} \lambda_{j} m_{j} z_{j}\right)+\sigma_{2}^{2}\left(\sum \lambda_{l}\right)^{2}
\end{aligned}
$$


Integration of (A.5.2) over the surface of the hypersphere and subsequent division by the surface area $\Psi^{-1}$ yields

$$
\begin{aligned}
V o f V(r) & =\sigma_{4} \sum_{i=1}^{k} \lambda_{i}^{2}+2 \sigma_{22} \sum_{\substack{i=1 \\
i<j}}^{k} \sum_{j=1}^{k} \lambda_{i} \lambda_{j}-\sigma_{2}^{2}\left(\sum_{i=1}^{k} \lambda_{i}\right)^{2}+4 \sigma_{2}\left(\sum_{i=1}^{k} \lambda_{i}^{2} m_{i}^{2}\right) \\
& =\frac{3 r^{4}}{k(k+2)} \sum \lambda_{i}^{2}+\frac{2 r^{4}}{k(k+2)} \sum_{i<j} \lambda_{i} \lambda_{j}-\frac{r^{4}}{k^{2}}\left(\sum \lambda_{i}\right)^{2}+4 \frac{r^{2}}{k}\left(\sum \lambda_{i}^{2} m_{i}^{2}\right)
\end{aligned}
$$

where $\sigma_{4}=\frac{3 r^{4}}{k(k+2)}$ and $\sigma_{22}=\frac{r^{4}}{k(k+2)}$ are the fourth order spherical region moments defined in section 3.2.3. The expression for the VofV on a radius $r$ given by equation (3.2.15) follows easily from this result upon recognizing that $\left(\Sigma \lambda_{i}\right)^{2}=\Sigma \lambda_{i}^{2}+2 \sum_{i<j} \lambda_{i} \lambda_{j}$. 


\section{Appendix A.6}

\section{Derivation of the Spherical Variance Under Case III for $k=2$ Variables}

A general form for the spherical variance when the model is second order is given by (3.2.16),

$$
V^{r}=\operatorname{tr}\left\{S\left(X^{\prime} X\right)^{-1}\right\}
$$

In the case where there are two independent variables present in the model the product of the spherical region moment matrix $S$ and the $6 \times 6$ symmetric matrix $\left(X^{\prime} X\right)^{-1}$ is

$$
S\left(X^{\prime} X\right)^{-1}=\left[\begin{array}{cccccc}
1 & 0 & 0 & \sigma_{2} & \sigma_{2} & 0 \\
0 & \sigma_{2} & 0 & 0 & 0 & 0 \\
0 & 0 & \sigma_{2} & 0 & 0 & 0 \\
\sigma_{2} & 0 & 0 & \sigma_{4} & \sigma_{22} & 0 \\
\sigma_{2} & 0 & 0 & \sigma_{22} & \sigma_{4} & 0 \\
0 & 0 & 0 & 0 & 0 & \sigma_{22}
\end{array}\right]\left[\begin{array}{cccccc}
c^{00} & c^{01} & c^{02} & c^{03} & c^{04} & c^{05} \\
c^{01} & c^{11} & c^{12} & c^{13} & c^{14} & c^{15} \\
c^{02} & c^{12} & c^{22} & c^{23} & c^{24} & c^{25} \\
c^{03} & c^{13} & c^{23} & c^{33} & c^{34} & c^{35} \\
c^{04} & c^{14} & c^{24} & c^{34} & c^{44} & c^{45} \\
c^{05} & c^{15} & c^{25} & c^{35} & c^{45} & c^{55}
\end{array}\right]
$$

The element in the $i$-th row and $j$-th column of $\left(X^{\prime} X\right)^{-1}$ is denoted by $c^{\mathrm{ij}}$ where the numbering of the rows and columns begins with zero.

Since the trace of the resulting matrix is the quantity of interest, only the diagonal elements of $S\left(X^{\prime} X\right)^{-1}$ are presented here. The diagonal elements are given by row and column below. 


$\begin{array}{ccc}\text { Row } & \text { Column } & \text { Diagonal Element } \\ 0 & 0 & c^{00}+\sigma_{2}\left(c^{03}+c^{04}\right) \\ 1 & 1 & \sigma_{2} c^{11} \\ 2 & 2 & \sigma_{2} c^{22} \\ 3 & 3 & \sigma_{2} c^{03}+\sigma_{4} c^{33}+\sigma_{22} c^{34} \\ 4 & 4 & \sigma_{2} c^{04}+\sigma_{22} c^{34}+\sigma_{4} c^{44} \\ 5 & 5 & \sigma_{22} c^{55}\end{array}$

The trace of the matrix is the sum of the diagonal elements. Hence, gathering like terms, the spherical variance for a second order model in two variables is

$$
V^{r}=c^{00}+\sigma_{2}\left[\sum_{i=1}^{2}\left(2 c^{0, i+2}+c^{i i}\right)\right]+\sigma_{4} \sum_{i=1}^{2} c^{i+2, i+2}+\sigma_{22}\left(c^{55}+2 c^{34}\right)
$$




\section{Appendix A.7}

\section{Program to Generate the VofV Under Case III for $k=2$ Variables}

A computer macro which evaluates the VofV as given by equation (3.2.17) for a second order model and design in two variables is presented in this section. The macro is written in the SAS programming language using the MATRIX procedure.

* This SAS macro computes the spherical variance and the VofV *;

* for second order designs in two variables.

* The program is formulated using the MATRIX procedure in SAS *;

* The spherical variance and the VofV are computed for a range *;

* of radii, from $r=0$ to $r=1.40$ in this case. *;

$$
\begin{aligned}
& \text { MACRO COMPUTE } \\
& \text { PROC MATRIX } \\
& \text { FETCH X DATA = DESIGN (DROP = DESIGN); } \\
& \text { FETCH DESIGN DATA = DESIGN }(\text { KEEP = DES } \\
& \quad \text { SRAD = J(1,21); } \\
& \quad \text { SVR = J(1,21); } \\
& \quad \text { SVOV = J }(1,21) ; \\
& \text { N }=\text { NROW }(X) ; \\
& X=J(N, 1) \| X ; \\
& X P X=X^{\prime *} X ; \\
& C=I N V(X P X) ;
\end{aligned}
$$$$
\text { FETCH DESIGN DATA = DESIGN }(\text { KEEP = DESIGN }) \text {; }
$$ 
* $\mathrm{N}$ is the number of design points.

* $\mathrm{X}$ is the model-matrix for the design.

* $C$ is the inverse of the $X^{\prime} X$ matrix.

* An element of $C$ is designated by $C(i, j)$

* for rows,columns $i, j=1,2,3,4,5,6$.
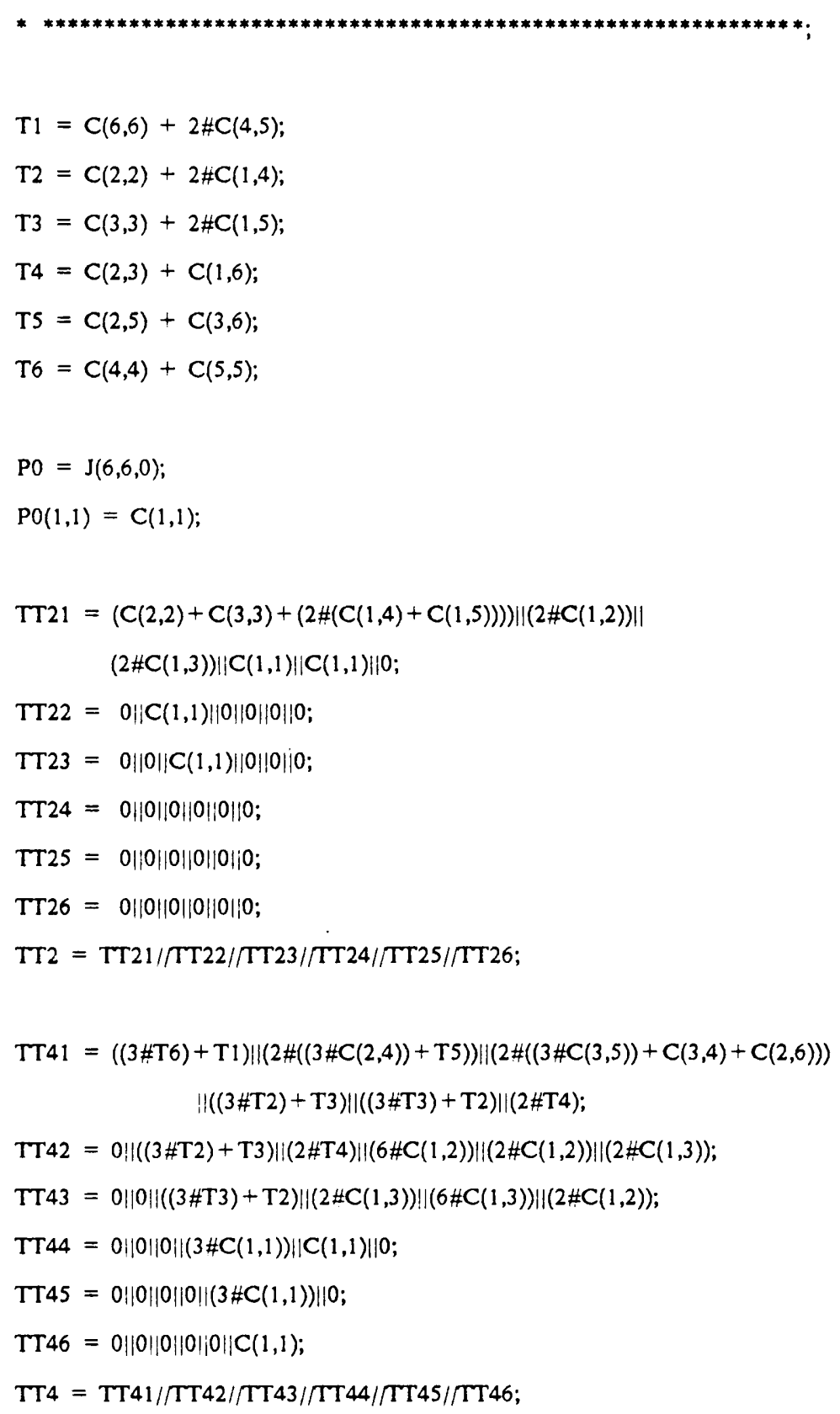


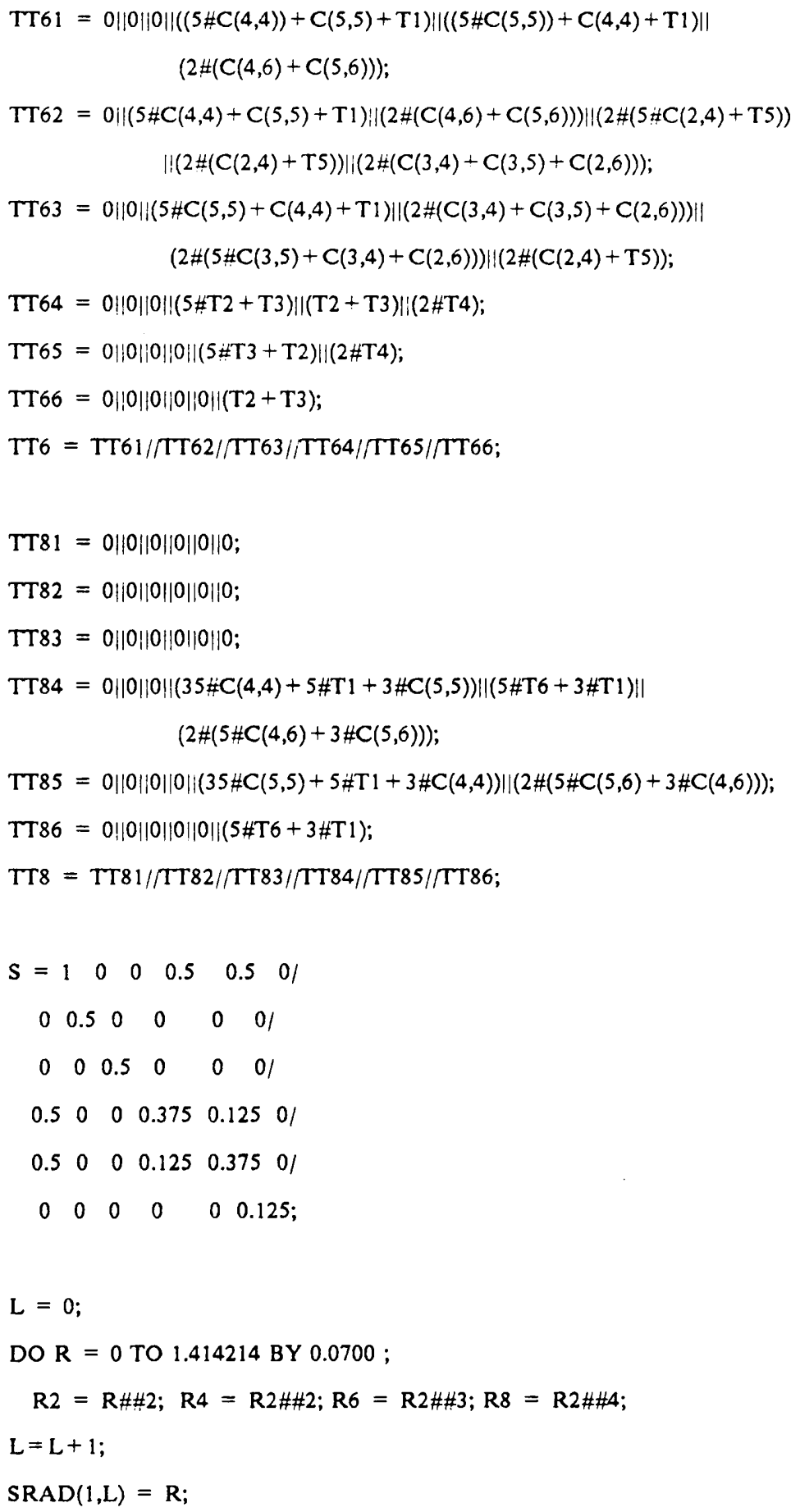


* Calculation of the spherical variance -- VR.

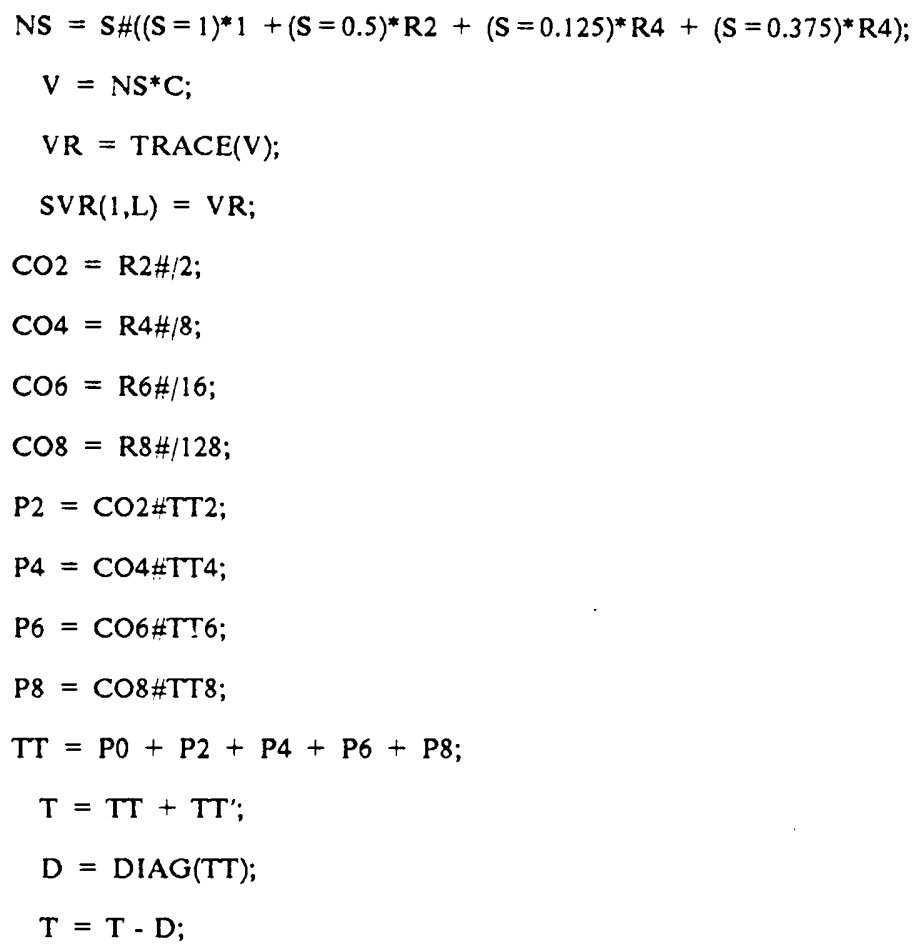

* NS is the spherical region moment matrix evaluated at $R$.

* For each radius, $\mathrm{R}$, the corresponding VOFV is put in SVOV.

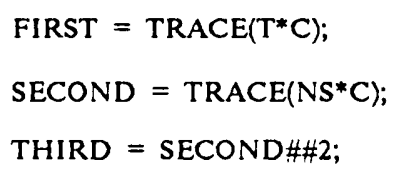


VOFV $=\operatorname{TRACE}\left(T^{*} \mathrm{C}\right)-\left(\operatorname{TRACE}\left(\mathrm{NS}^{*} \mathrm{C}\right)\right) \# \# 2$

CK_VOFV $=$ TRACE $\left(T^{*} C\right)-(V R) \# \# 2 ;$

* $* * * * * * * * * * * * * * * * * * * * * * * * * * * * * * * * * * * * * * * * * * * * * * * * * * * * * * * * * * * * * *$.

* CK_VOFV is an alternative way to compute the VorV.

$\operatorname{SVOV}(1, \mathrm{~L})=\operatorname{VOFV}$

END;

VAR = DESIGN ||$N|| S V R ;|| S V O V ;$

OUTPUT VAR OUT = STAT;

PROC PRINT;

$\%$ 
* The SAS data statements necessary to create the data set *;

* named DESIGN appear below.

*;

* X1, X2 are the independent variables; X1SQ, X2SQ and X1X2 *;

* define the remaining columns of the model-matrix $X$. *;

* The column of ones is affixed to the model-matrix within *;

* the macro.

* *;

* The design may be identified by the value of the variable DESIGN. * *;

* An example set of design points is also given. *;

\section{DATA DESIGN;}

INPUT X1 X2;

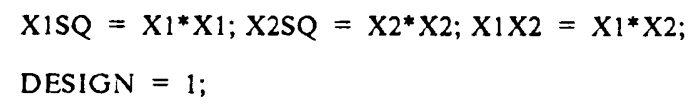

\section{CARDS;}

11

$1-1$

$-1$

$-1 \quad-1$

$-1.4142135620$

$1.414213562 \quad 0$

$0 \quad-1.414213562$

$0 \quad 1.414213562$

$0 \quad 0$

$0 \quad 0$

COMPUTE;

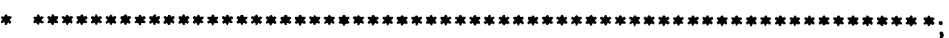

* The command COMPUTE calls the macro, thereby, generating the *;

* spherical variances and VorVs for this design. *;

* They may be found in the new data set STAT. *; 


\section{Appendix B}

\section{Appendices for Chapter IV}

\section{Appendix B.1}

\section{Proof of Lemma 4.1.1}

The Lagrangian multiplier, $\mu$, involved in the optimization of $\operatorname{Var}(\hat{y}(\underline{x})) / \sigma^{2}$ under the conditions of case II is related to the radius, $r$, of the hypersphere by

$$
r=\left[\sum_{i=1}^{k}\left(\frac{\lambda_{i} m_{i}}{\lambda_{i}-\mu}\right)^{2}\right] \frac{1}{2}=f(\mu)
$$

Lemma 4.1.1 pertains to the behavior of this relationship for values of $\mu=\mu_{\max }>\lambda_{k}$ and $\mu=\mu_{\min }<\lambda_{1}$. For $\mu=\mu_{\max }>\lambda_{k}$ the function $f$ is continuous and decreasing. Thus, the inverse function exists and is continuous. By a well known theorem from calculus, (see, for example, Swokowski (1979)). 


$$
\frac{\partial \mu_{\max }}{\partial r}=\frac{1}{\frac{\partial r}{\partial \mu_{\max }}}
$$

for $\mu=\mu_{\max }>\lambda_{k}$.

Similarly, for values of $\mu=\mu_{\min }<\lambda_{1}, f$ is continuous and increasing and, therefore, the inverse function of $f$ exists. Thus, for $\mu=\mu_{\min }<\lambda_{1}$,

$$
\frac{\partial \mu_{\min }}{\partial r}=\frac{1}{\frac{\partial r}{\partial \mu_{\min }}}
$$

Now, in either case, differentiation of $f(\mu)$ with respect to $\mu$ yields

$$
\frac{\partial r}{\partial \mu}=\left[\sum_{i=1}^{k}\left(\frac{\lambda_{i} m_{i}}{\lambda_{i}-\mu}\right)^{2}\right]-\frac{1}{2}\left[\sum_{i=1}^{k} \frac{\left(\lambda_{i} m_{i}\right)^{2}}{\left(\lambda_{i}-\mu\right)^{3}}\right] .
$$

Therefore,

$$
\frac{\partial \mu}{\partial r}=\left[\sum_{i=1}^{k}\left(\frac{\lambda_{i} m_{i}}{\lambda_{i}-\mu}\right)^{2}\right] \frac{1}{2}\left[\sum_{i=1}^{k} \frac{\left(\lambda_{i} m_{i}\right)^{2}}{\left(\lambda_{i}-\mu\right)^{3}}\right]^{-1}
$$

for $\mu=\mu_{\max }>\lambda_{k}$ or $\mu=\mu_{\min }<\lambda_{1}$.

Utilizing these results, the lemma may now be proved.

Proof of Lemma 4.1.1: Recall that $0<\lambda_{1} \leq \lambda_{2} \leq \ldots \leq \lambda_{k}$. Then, it is obvious from equation (B.1.2) that,

i) if $\mu=\mu_{\max }>\lambda_{k}$,

$$
\frac{\partial \mu_{\max }}{\partial r}<0 \text {, and, }
$$


ii) if $\mu=\mu_{\min }<\lambda_{1}$,

$\frac{\partial \mu_{\min }}{\partial r}>0$.

Appendix B. 


\section{Appendix B.2}

\section{Proof of Lemma 4.2.1}

Consider a translation of the axis system of the $x$-design variables to the axis system of the $z$-variables as set forth in section 4.2.2. That is, $z_{i}=x_{i}+m_{i}$ for some $m_{i}$ and $i=1,2, \ldots, k$. To prove Lemma 4.2 .1 it is necessary to establish the existence of matrices $L$ which satisfy the conditions of the lemma in the first and second order model cases.

In general notation, the model in terms of the $x$-variables may be written as

$$
\underline{y}=X \underline{B}+\underline{\varepsilon}
$$

where $X$ is the appropriate model-matrix. In terms of the translated $z$-variables the model is

$$
\underline{y}=Z_{\underline{Y}}+\underline{\varepsilon}
$$

for appropriate model-matrix $Z$. The two models are equivalent.

First consider fitting a first order model to the response with a design in $k$ variables. In this case, the model-matrices and parameter vectors of the models have the forms 


$$
X=\left[\begin{array}{cccc}
1 & x_{11} & \ldots & x_{1 k} \\
1 & x_{21} & \ldots & x_{2 k} \\
\cdot & \cdot & & \cdot \\
\cdot & \cdot & \ldots & \cdot \\
\cdot & \cdot & & \cdot \\
1 & x_{N 1} & \ldots & x_{N k}
\end{array}\right], \beta=\left[\begin{array}{c}
\beta_{0} \\
\beta_{1} \\
\cdot \\
\cdot \\
\cdot \\
\beta_{k}
\end{array}\right]
$$

and

$$
Z=\left[\begin{array}{cccc}
1 & x_{11}+m_{1} & \ldots & x_{1 k}+m_{k} \\
1 & x_{21}+m_{1} & \ldots & x_{2 k}+m_{k} \\
\cdot & \cdot & & \cdot \\
\cdot & \cdot & & \cdot \\
\cdot & \cdot & & \cdot \\
1 & x_{N 1}+m_{1} & \ldots & x_{N k}+m_{k}
\end{array}\right], \underline{\gamma}=\left[\begin{array}{c}
\gamma_{0} \\
\gamma_{1} \\
\cdot \\
\cdot \\
\cdot \\
\gamma_{k}
\end{array}\right]
$$

The vector of parameters, $\underline{\underline{y}}$, is readily seen to be related to the vector $\underline{\beta}$ in the following manner:

$$
\underline{\gamma}=\left[\begin{array}{c}
\beta_{0}-m_{1} \beta_{1}-m_{2} \beta_{2}-\cdots-m_{k} \beta_{k} \\
\beta_{1} \\
\beta_{2} \\
\cdot \\
\cdot \\
\cdot \\
\beta_{k}
\end{array}\right]
$$

This may be written as

$$
\chi=G \beta
$$

for 


$$
G=\left[\begin{array}{ccccc}
1 & -m_{1} & -m_{2} & \ldots & -m_{k} \\
0 & 1 & 0 & \ldots & 0 \\
0 & 0 & 1 & \ldots & 0 \\
. & . & . & & \cdot \\
\cdot & . & . & \ldots & \cdot \\
. & . & . & & . \\
0 & 0 & 0 & \ldots & 1
\end{array}\right] .
$$

The matrix $G$ is a $p \times p$ matrix of rank $p$, where $p=k+1$, the number of parameters in the model. Each row of $\chi=G \beta$ is an estimable function of $\beta$. Thus, the model in terms of the $x$-variables may be reparameterized as (see Graybill (1976), p. 493)

$$
\begin{aligned}
\underline{\nu} & =X \underline{\beta}+\underline{\varepsilon} \\
& =X G^{-1} \underline{\gamma}+\underline{\varepsilon}
\end{aligned}
$$

which is equivalent to the model

$$
\underline{\underline{y}}=Z_{\underline{\gamma}}+\underline{\varepsilon}
$$

Therefore,

$$
Z=X G^{-1}
$$

Thus, choose the matrix $L$ of the lemma to be

$$
L=G^{-1}=\left[\begin{array}{ccccc}
1 & m_{1} & m_{2} & \ldots & m_{k} \\
0 & 1 & 0 & \ldots & 0 \\
0 & 0 & 1 & \ldots & 0 \\
. & . & . & & . \\
. & . & . & & . \\
. & . & . & & . \\
0 & 0 & 0 & \ldots & 1
\end{array}\right] .
$$

It is easily verified that $Z=X L$ for thee model-matrices $X$ and $Z$ of a first order model. 
Note that

$$
L^{\prime} \underline{x}^{*}=L^{\prime}\left[\begin{array}{c}
1 \\
x_{1} \\
x_{2} \\
\cdot \\
\cdot \\
x_{k}
\end{array}\right]=\left[\begin{array}{c}
1 \\
x_{1}+m_{1} \\
x_{2}+m_{2} \\
\cdot \\
\cdot \\
x_{k}+m_{k}
\end{array}\right]=\underline{z}^{*}
$$

for any point $\underline{x}=\left(\begin{array}{lllll}x_{1} & x_{2} & \ldots & x_{k}\end{array}\right)^{\prime}$ and $\underline{z}=\left(\begin{array}{llll}x_{1}+m_{1} & x_{2}+m_{2} & \ldots & x_{k}+m_{k}\end{array}\right)^{\prime}$.

Now consider the case of fitting a second order model to the response. In this case, the model-matrices $X$ and $Z$ and their associated parameter vectors of the model are

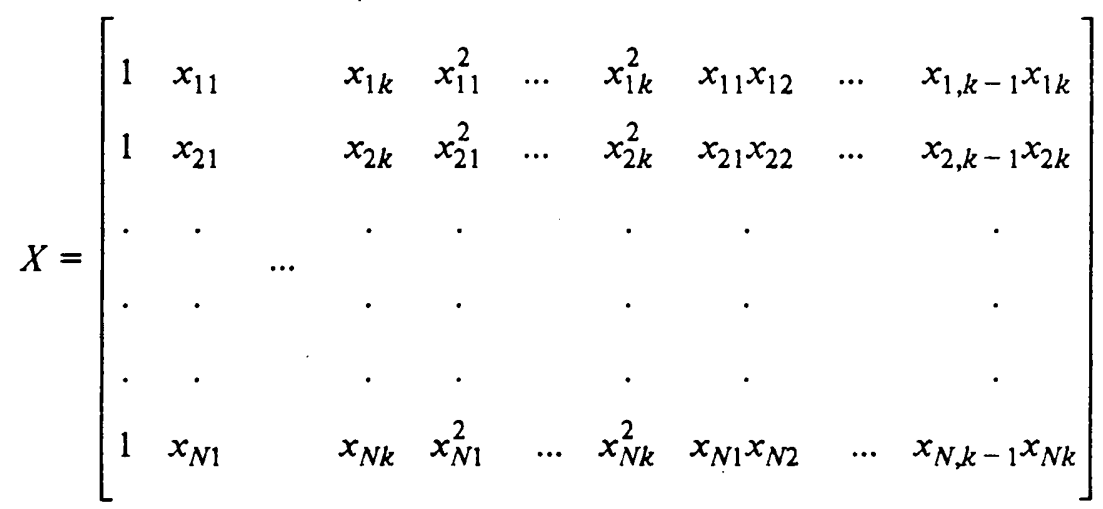

$Z$ has the same form as $X$ with the values $x_{i j}$ replaced by $z_{i j}=x_{i j}+m_{j}$. The vectors of parameters in this case are

$$
\beta=\left(\begin{array}{llllllllllll}
\beta_{0} & \beta_{1} & \ldots & \beta_{k} & \beta_{11} & \ldots & \beta_{k k} & \beta_{12} & \beta_{13} & \ldots & \beta_{k-1, k}
\end{array}\right)^{\prime}
$$

and

$$
\boldsymbol{\gamma}=\left(\begin{array}{lllllllllll}
\gamma_{0} & \gamma_{1} & \ldots & \gamma_{k} & \gamma_{11} & \ldots & \gamma_{k k} & \gamma_{12} & \gamma_{13} & \ldots & \gamma_{k-1, k}
\end{array}\right)^{\prime}
$$

Here the number of parameters in the model is $p=2 k+\left(\begin{array}{c}k \\ 2\end{array}\right)+1$. 
As in the first order case, the vector $\mathcal{Y}$ is a reparameterization of $\underline{\beta}$ of the form $\chi=G ß$. It can be shown that the matrix $G$ of the reparameterization is given by

$$
G=\left[\begin{array}{cccc}
1 & -\underline{m^{\prime}} & \underline{m 2^{\prime}} & \underline{m 3^{\prime}} \\
\underline{0} & I & -T & -M \\
\underline{0} & O & I & O \\
\underline{0} & O & O & I
\end{array}\right]
$$

where $O$ and $\underline{0}$ are a matrix and vector with all elements equal to zero; and $I$ represents the identity matrix.

$$
\begin{aligned}
\underline{m}^{\prime} & =\left(\begin{array}{llll}
m_{1} & m_{2} & \ldots & m_{k}
\end{array}\right), \\
\underline{m 2^{\prime}} & =\left(\begin{array}{llll}
m_{1}^{2} & m_{2}^{2} & \ldots & m_{k}^{2}
\end{array}\right), \\
\underline{m 3^{\prime}} & =\left(\begin{array}{lllll}
m_{1} m_{2} & m_{1} m_{3} & \ldots & m_{k-1} m_{k}
\end{array}\right) .
\end{aligned}
$$

The matrix $T$ is a diagonal $k \times k$ matrix with the element in the $i$-th row and $i$-th column equal to $2 m_{i}$. The $k \times\left(\begin{array}{l}k \\ 2\end{array}\right)$ matrix $M$ is

$$
M=\left[\begin{array}{ccccccc}
\beta_{12} & \beta_{13} & \cdots & \beta_{1 k} & \beta_{23} & \cdots & \beta_{k-1, k} \\
m_{2} & m_{3} & \cdots & m_{k} & 0 & \cdots & 0 \\
m_{1} & 0 & \cdots & 0 & m_{3} & \cdots & 0 \\
0 & m_{1} & \cdots & 0 & m_{2} & \cdots & 0 \\
0 & 0 & \cdots & 0 & 0 & \cdots & 0 \\
. & . & & . & . & & . \\
. & . & & . & . & & . \\
. & . & & . & . & & . \\
0 & 0 & & 0 & 0 & \cdots & m_{k} \\
0 & 0 & \ldots & m_{1} & 0 & \cdots & m_{k-1}
\end{array}\right]
$$

(each column is identified by the corresponding element of $\underline{\beta}$ to which it will be multiplied in the product $G ß)$ ). $G$ is a $p \times p$ matrix of rank $p$, therefore, $G^{-1}$ exists. 
Now, with $X, Z, \beta, \underline{\gamma}$ described above for the second order case, and $G$ given by (B.2.1), the model is

$$
\begin{aligned}
y & =X \underline{\beta}+\underline{\varepsilon} \\
& =X G^{-1} \underline{\gamma}+\varepsilon .
\end{aligned}
$$

This is equivalent to the model in terms of the $z$-variables, thus, it must be that

$$
Z=X G^{-1}
$$

Let $L=G^{-1}$, then $L$ is a $p \times p$ matrix of rank $p$ for which

$$
Z=X L
$$

This result and the result that $\underline{z}^{*}=L^{\prime} \underline{x}^{*}$ are easily verified by multiplication of matrices. For a second order model

$$
\underline{x}^{*}=\left(\begin{array}{lllllllllll}
1 & x_{1} & \ldots & x_{k} & x_{1}^{2} & \ldots & x_{k}^{2} & x_{1} x_{2} & x_{1} x_{3} & \ldots & x_{k-1} x_{k}
\end{array}\right)^{\prime},
$$

for any point $\underline{x}=\left(\begin{array}{lllll}x_{1} & x_{2} & \ldots & x_{k}\end{array}\right)^{\prime}$ in the space of the $x$-variables. The vector $\underline{z}^{*}$ has the same form with $x_{i}$ replaced by $z_{i}=x_{i}+m_{i}$ for $i=1,2, \ldots, k$. The matrix $L$ of the lemma is given below.

$$
L=\left[\begin{array}{cccc}
1 & \underline{m}^{\prime} & \underline{m 2^{\prime}} & \underline{m 3^{\prime}} \\
\underline{0} & I & T & M \\
\underline{0} & O & I & O \\
\underline{0} & O & O & I
\end{array}\right] .
$$




\section{Appendix B.3}

\section{Proof of Theorem 4.2.5}

In this section it will be shown that under the conditions stated in Theorem 4.2 .5 the form of the RofV for a second order model and design is invariant to the number of center points, $n_{0}$, of the design. The proof for the result that the VofV is also invariant to $n_{0}$ in this case closely follows that for the RofV. It will not be presented here.

Let $N=n+n_{0}$ denote the number of design points in a design used to fit a second order model to the response of a system. The design has $n_{0}$ points at the center of the region of the $k$ design variables, $x_{1}, x_{2}, \ldots, x_{k}$. Without loss of generality, suppose the center of the design variables is at $\underline{x}=\underline{0}$. Also, suppose the four conditions of Theorem 4.2.5 hold for the design.

Under the conditions of 4.2.5, the $X^{\prime} X$ matrix has the form given below.

$$
X^{\prime} X=\left[\begin{array}{llll}
N & \underline{0}^{\prime} & \underline{a}^{\prime} & \underline{0}^{\prime} \\
\underline{0} & A & O & O \\
\underline{a} & O & B & O \\
\underline{0} & O & O & C
\end{array}\right]
$$

where

$$
\begin{aligned}
\underline{a} & =\left(\begin{array}{llll}
\sum_{u=1}^{N} x_{u 1}^{2} & \sum_{u=1}^{N} x_{u 2}^{2} & \ldots & \sum_{u=1}^{N} x_{u k}^{2}
\end{array}\right)^{\prime} \\
& =\left(\begin{array}{llll}
a & a & \ldots & a)^{\prime}
\end{array}{ }^{\prime}\right. \text { by condition ii) }
\end{aligned}
$$


The matrix $A$ is a $k \times k$ diagonal matrix. The diagonal elements are $N \cdot$ [ii], which are all equal to $a$ by condition ii). The off-diagonal elements of $A$ are $N$ times the second mixed moments of the design, and, hence, equal to zero. Thus, $A$ is of the form

$$
A=\text { diagonal }(a, a, \ldots, a)
$$

The matrix $B$ consists of $N$ times the fourth pure moments on the diagonal and $N$ times the even fourth mixed moments on the off-diagonal positions. By application of conditions iii) and iv), the matrix $B$ is a $k \times k$ symmetric matrix of the form

$$
B=\left[\begin{array}{lllll}
b & c & c & \ldots & c \\
c & b & c & \ldots & c \\
\cdot & \cdot & \cdot & & \cdot \\
\cdot & \cdot & \cdot & & \cdot \\
\cdot & \cdot & \cdot & & \cdot \\
c & c & c & \ldots & b
\end{array}\right]=(b-c) I+c 11^{\prime}
$$

where 1 is a $k \times 1$ vector of ones. The $\left(\begin{array}{l}k \\ 2\end{array}\right) \times\left(\begin{array}{l}k \\ 2\end{array}\right)$ matrix $\mathrm{C}$ is a diagonal matrix with diagonal elements equal to $c$ by condition iv). The elements in the off-diagonal positions of $C$ are odd fourth moments and, consequently, equal to zero by condition i). That is,

$$
C=\text { diagonal }(c, c, \ldots, c)
$$

Again, $O$ and $\underline{Q}$ represent a matrix and vector with all elements equal to zero.

To calculate the prediction variance it is necessary to determine the inverse of $X^{\prime} X$. Before proceeding some matrix calculations which will be required in the formulation of $\left(X^{\prime} X\right)^{-1}$ and the prediction variance are given.

1) The inverse of the matrix $B$ exists and has the form 


$$
\begin{aligned}
B^{-1} & =b^{*} I+c^{*} \underline{1} \underline{1}^{\prime} \\
& =\left[\begin{array}{ccccc}
b^{*} & c^{*} & c^{*} & \ldots & c^{*} \\
c^{*} & b^{*} & c^{*} & \ldots & c^{*} \\
\cdot & \cdot & \cdot & & \cdot \\
\cdot & \cdot & \cdot & & \cdot \\
\cdot & \cdot & \cdot & & \cdot \\
c^{*} & c^{*} & c^{*} & \ldots & b^{*}
\end{array}\right] .
\end{aligned}
$$

for $b^{*}=\frac{1}{b-c}$ and $c^{*}=\frac{-c /(b-c)}{b+(k-1) c}$.

2) By an application of the Sherman-Morrison-Woodbury theorem (see section 4.3)

$$
\left(B-\frac{\underline{a} \underline{a}^{\prime}}{N}\right)^{-1}=B^{-1}-\frac{B^{-1} \underline{a} \underline{a}^{\prime} B^{-1}}{N-\underline{a}^{\prime} B^{-1} \underline{a}} .
$$

3)

$$
\begin{aligned}
a B^{-1} & =\left(a\left(b^{*}+(k-1) c^{*}\right) \ldots a\left(b^{*}+(k-1) c^{*}\right)\right) \\
& =\left(\begin{array}{llll}
a^{*} & a^{*} & \ldots & a^{*}
\end{array}\right) .
\end{aligned}
$$

with $B^{-1}$ defined by calculation 1 ).

By the repeated application of the inverse of a symmetric pattern matrix, the inverse of $X^{\prime} X$ in equation (B.3.1) is

$$
\left(X^{\prime} X\right)^{-1}=\left[\begin{array}{cccc}
\left(N-\underline{a}^{\prime} B^{-1} \underline{a}^{\prime}\right)^{-1} & \underline{0}^{\prime} & -\left(N-\underline{a}^{\prime} B^{-1} \underline{a}\right)^{-1} \underline{a}^{\prime} B^{-1} & \underline{0}^{\prime} \\
\underline{0} & A^{-1} & O & 0 \\
-\left(N-\underline{a}^{\prime} B^{-1} \underline{a}\right)^{-1} B^{-1} \underline{a} & O & \left(B-\frac{\underline{a} \underline{a}^{\prime}}{N}\right)^{-1} & O \\
0 & O & O & C^{-1}
\end{array}\right]
$$


Now, for a second order model the prediction variance at a location $\underline{x}=\left(\begin{array}{llll}x_{1} & x_{2} & \ldots & x_{k}\end{array}\right)^{\prime}$ with

$$
\begin{aligned}
\underline{x}^{* \prime} & =\left(\begin{array}{llllllllll}
1 & x_{1} & \ldots & x_{k} & x_{1}^{2} & \ldots & x_{1} x_{2} & x_{1} x_{3} & \ldots & x_{k-1} x_{k}
\end{array}\right) \\
& =\left(\begin{array}{llllll}
1 & \underline{x}^{\prime} & \underline{x 2^{\prime}} & \underline{x x^{\prime}}
\end{array}\right)
\end{aligned}
$$

where

$$
\underline{x 2^{\prime}}=\left(\begin{array}{llll}
x_{1}^{2} & x_{2}^{2} & \ldots & x_{k}^{2}
\end{array}\right)
$$

and

$$
\underline{x x^{\prime}}=\left(\begin{array}{llll}
x_{1} x_{2} & x_{1} x_{3} & \ldots & x_{k-1} x_{k}
\end{array}\right),
$$

is

$$
\begin{aligned}
\frac{1}{\sigma^{2}} \operatorname{Var}(\hat{y}(\underline{x}))= & \underline{x}^{*}\left(X^{\prime} X\right)^{-1} \underline{x}^{*} \\
= & \left(N-\underline{a}^{\prime} B^{-1} \underline{a}\right)^{-1}+\underline{x^{\prime}} A^{-1} \underline{x}-2\left(N-\underline{a^{\prime}} B^{-1} \underline{a}\right)^{-1} \underline{a^{\prime}} B^{-1} \underline{x 2} \\
& +\underline{x \underline{x}^{\prime}}\left(B-\frac{\underline{a} \underline{a^{\prime}}}{N}\right)^{-1} \underline{x}+\underline{x x^{\prime}} C^{-1} \underline{x x} .
\end{aligned}
$$

Note that from calculation 3), 


$$
\begin{aligned}
a^{\prime} B^{-1} \underline{x 2} & =\left(\begin{array}{llll}
a^{*} & a^{*} & \ldots & a^{*}
\end{array}\right)\left[\begin{array}{c}
x_{1}^{2} \\
x_{2}^{2} \\
\cdot \\
\cdot \\
\cdot \\
x_{k}^{2}
\end{array}\right] \\
& =a^{*} \sum_{i=1}^{k} x_{i}^{2} \\
& =a^{*} r^{2}
\end{aligned}
$$

since $\sum_{i=1}^{k} x_{i}^{2}=\underline{x}^{\prime} \underline{x}=r^{2}$, where $r$ is the radius of the sphere on which the point $\underline{x}$ lies. This result and the result of calculation 2) yields the following expression for the prediction variance.

$$
\begin{aligned}
\frac{1}{\sigma^{2}} \operatorname{Var}(\hat{y}(\underline{x}))= & \left(N-\underline{a}^{\prime} B^{-1} \underline{a}\right)^{-1}+\underline{x}^{\prime} A^{-1} \underline{x} \\
& -2\left(N-\underline{a}^{\prime} B^{-1} \underline{a}\right)^{-1} a^{*} r^{2}+\underline{x 2^{\prime}} B^{-1} \underline{x 2} \\
& -\left(N-\underline{a}^{\prime} B^{-1} \underline{a}\right)^{-1}\left(a^{*} r^{2}\right)^{2}+\underline{x x^{\prime}} C^{-1} \underline{x x} .
\end{aligned}
$$

Consider the RofV in this case on the surface of a hypersphere of radius $r$. Let $\underline{x}_{\max }$ and $\underline{x}_{\min }$ represent the locations of the maximum and minimum prediction variances respectively on the surface of the sphere. Note that

$$
\underline{x}_{\max }^{\prime} \underline{x}_{\max }=\sum_{i=1}^{k} x_{i, \max }^{2}=r^{2}
$$

and

$$
\underline{x}_{\min }^{\prime} \underline{x}_{\min }=\sum_{i=1}^{k} x_{i, \min }^{2}=r^{2}
$$


Then, the range of the prediction variances on the surface of the sphere is

$$
\begin{aligned}
\operatorname{Rof} V(r)= & \frac{1}{\sigma^{2}} \operatorname{Var}\left(\hat{y}\left(\underline{x}_{\max }\right)\right)-\frac{1}{\sigma^{2}} \operatorname{Var}\left(\hat{y}\left(\underline{x}_{\min }\right)\right) \\
= & \left\{\underline{x}_{\max }^{\prime} A^{-1} \underline{x}_{\max }-2\left(N-\underline{a}^{\prime} B^{-1} \underline{a}\right)^{-1} a^{*} r^{2}\right. \\
& +\underline{x 2}_{\max }^{\prime} B^{-1} \underline{x 2}_{\max }-\left(N-\underline{a}^{\prime} B^{-1} \underline{a}\right)^{-1}\left(a^{*} r^{2}\right)^{2} \\
& \left.+\underline{x x}_{\max }^{\prime} C^{-1} \underline{x x}_{\max }\right\} \\
& -\left\{\underline{x}_{\min }^{\prime} A^{-1} \underline{x}_{\min }-2\left(N-\underline{a}^{\prime} B^{-1} \underline{a}\right)^{-1} a^{*} r^{2}\right. \\
& +\underline{x 2}_{\min }^{\prime} B^{-1} \underline{x 2}_{\min }-\left(N-\underline{a}^{\prime} B^{-1} \underline{a}\right)^{-1}\left(a^{*} r^{2}\right)^{2} \\
& \left.+\underline{x x}_{\min }^{\prime} C^{-1} \underline{x x} \underline{\min }_{\min }\right\} .
\end{aligned}
$$

This follows from equation (B.3.2) with substitution of $\underline{x}_{\max }$ and $\underline{x}_{\min }$ for $\underline{x}$ where appropriate. The obvious simplification yields

$$
\begin{aligned}
& \operatorname{Rof} V(r)=\underline{x}_{\max }^{\prime} A^{-1} \underline{x}_{\max }+\underline{x 2^{\prime}} B^{-1} \underline{x 2}+\underline{x x^{\prime}} C^{-1} \underline{x x}
\end{aligned}
$$

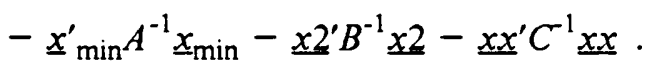

Note that the elements of the matrices $A, B$ and $C$ are sums of $x_{u i}, x_{u i}^{2}$ and $x_{u i} x_{u j}$ for $u=1,2, \ldots, N, i, j=1,2, \ldots, k$ where $x_{u i}$ is the $u$-th setting of the $i$-th variable used in the design. As such, the elements of $A, B$ and $C$, and, hence, those of $A^{-1}, B^{-1}$ and $C^{-1}$, do not depend on the value of $n_{0}$. Therefore, the RofV does not depend on $n_{0}$ in this case. That is, if the second order design satisfies the conditions of Theorem 4.2.5, the RofV is invariant to the number of center points of the design. 
The vita has been removed from the scanned document 